\title{
Pd(II)-Catalyzed Tandem Heterocyclization of 1-(1-Alkynyl)cyclopropyl Oxime Derivatives for the Synthesis of Functionalized Pyrroles
}

Dong Pan, Yin Wei and Min Shi*

State Key Laboratory of Organometallic Chemistry, Shanghai Institute of Organic Chemistry, Chinese Academy of Sciences, 345 Lingling Road, Shanghai 200032 China.

*Mshi@mail.sioc.ac.cn. Fax 86-21-64166128

\section{Supporting Information}

\section{Index}
(A) General Information
(B) General Procedure for the Preparation of Substrates
(C) General Procedure for the Pd-catalyzed Tandem Reaction of $\mathbf{1}$ and $\mathbf{4}$
(D) The Control Experiments
S8-S10
(E) The Transformation of Product
(F) Deuterium Labeling Experiments
(G) Spectroscopic Data of Substrates and Products
S19-S94
(H) References
S95 


\section{(A) General Information}

All reactions have been carried out under inert atmosphere in pre-dried glassware. The dry common solvents used were purified by an Innovative Technology PureSolv purification system. $\mathrm{MeOH}$ and $\mathrm{AcOH}$ were purified according to the standard methods. Commercially available chemicals were obtained from Acros Organics, Aldrich Chemical, Alfa Aesar, TCI, and used as received unless otherwise stated. Proton nuclear magnetic resonance $\left({ }^{1} \mathrm{H}\right.$ NMR) spectra and carbon nuclear magnetic resonance $\left({ }^{13} \mathrm{C} \mathrm{NMR}\right)$ spectra were recorded on an agilent-400 $\mathrm{MHz}$ spectrometer, and $\mathrm{CDCl}_{3}$ was purchased from Aldrich. Data are presented as follows: chemical shift (ppm), multiplicity $(\mathrm{s}=$ singlet, $\mathrm{d}=$ doublet, $\mathrm{t}=$ triplet, $\mathrm{q}=$ quartet, $\mathrm{sept}=$ septet, $\mathrm{m}=$ multiplet, $\mathrm{br}=$ broad $)$, coupling constants in Hertz $(\mathrm{Hz})$ and integration. Mass and High resolution mass spectra (HRMS) spectra were recorded by EI or ESI method. For thin-layer chromatography (TLC), silica gel plates (Huanghai GF254) were used. Flash column chromatography was performed on silica gel 60 (particle size 300-400 mesh ASTM, purchased from Yantai, China. 
(B) General Procedure for the Preparation of Substrates.

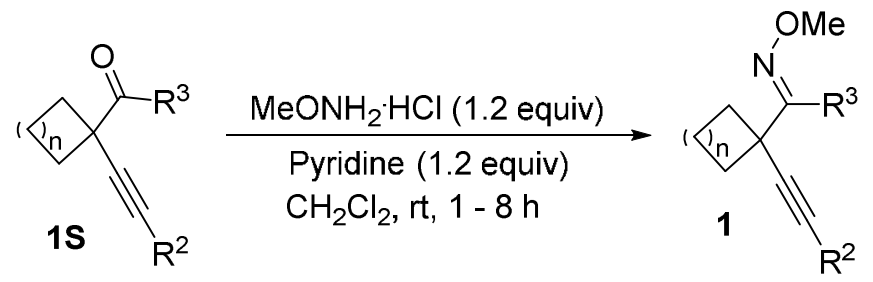

Scheme S1

\section{The substrates 1 were prepared according to Scheme $\mathrm{S1}:^{1}$}

The aldehydes or ketones $\mathbf{1 S}$ were synthesized according to the previously reported procedure. ${ }^{2}$ To a solution of aldehyde $\mathbf{1 S}$ (1.0 mmol, 1.0 equiv) and methoxyamine hydrochloride $(1.2 \mathrm{mmol}, 1.2$ equiv) in $\mathrm{CH}_{2} \mathrm{Cl}_{2}(3.0 \mathrm{~mL})$ was added dropwise pyridine (1.2 mmol, 1.2 equiv) at room temperature. After the resulting mixtures were stirred for 8 hours, $\mathrm{H}_{2} \mathrm{O}(30 \mathrm{~mL})$ was added and the mixture was extracted with $\mathrm{CH}_{2} \mathrm{Cl}_{2}(3 \times 10.0 \mathrm{~mL})$. The combined organic layers were washed with a saturated aqueous solution of $\mathrm{NaCl}$ and dried over anhydrous $\mathrm{Na}_{2} \mathrm{SO}_{4}$. Filtration, evaporation, and column chromatography on silica gel (eluent: petroleum ether/ethyl acetate 100:1) afforded the corresponding products $\mathbf{1 a - 1 1}$ and $\mathbf{1 r}-\mathbf{1 v}$.

\section{The substrates $1 \mathrm{~m}, 1 \mathrm{n}$ and 10 were prepared according to Scheme $\mathrm{S2}:^{3}$}

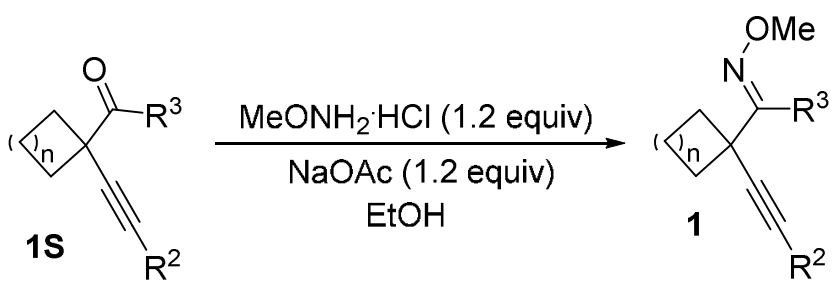

\section{Scheme S2}

To a solution of sodium acetate (1.2 mmol, 1.2 equiv) and methoxyamine hydrochloride (1.2 mmol, 1.2 equiv) in EtOH $(3.0 \mathrm{~mL})$ was added dropwise a solution of ketone or aldehyde $\mathbf{1 S}(1.2 \mathrm{mmol}$, 1.2 equiv) in $2 \mathrm{~mL} \mathrm{EtOH}$ at room temperature. After the resulting mixtures were stirred for 8 hours, $\mathrm{H}_{2} \mathrm{O}(30 \mathrm{~mL})$ was added and the mixture was extracted with $\mathrm{CH}_{2} \mathrm{Cl}_{2}(3 \times 10.0 \mathrm{~mL})$. The combined organic layers were washed with a saturated aqueous solution of $\mathrm{NaCl}$ and dried over anhydrous $\mathrm{Na}_{2} \mathrm{SO}_{4}$. Filtration, evaporation, and column chromatography on silica gel (eluent: petroleum 
ether/ethyl acetate $=100 / 1)$ afforded the corresponding products $\mathbf{1 m}, \mathbf{1 n}$ and $\mathbf{1 0}$.

\section{The substrates $1 p$ and $1 q$ were prepared according to ref $4 .^{4}$}

\section{The substrates 4 were prepared according to Scheme S3:}
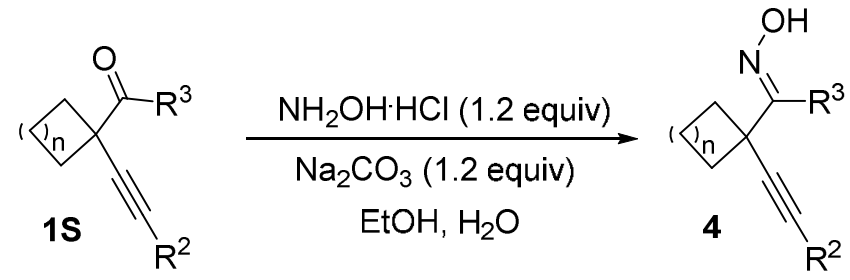

\section{Scheme S3}

To a solution of aldehydes (1.0 mmol, 1.0 equiv) and hydroxylamine hydrochloride (1.2 mmol, 1.2 equiv) in $\mathrm{EtOH}(3.0 \mathrm{~mL})$ was added dropwise a solution of $\mathrm{Na}_{2} \mathrm{CO}_{3}(1.2 \mathrm{mmol}, 1.2$ equiv) in $2 \mathrm{~mL}$ $\mathrm{H}_{2} \mathrm{O}$ at room temperature. After the resulting mixtures were stirred for 2 hours, the solvent was evaporated under reduced pressure, $\mathrm{H}_{2} \mathrm{O} 30 \mathrm{~mL}$ was added and the mixture was extracted with $\mathrm{CH}_{2} \mathrm{Cl}_{2}(3 \times 10.0 \mathrm{~mL})$. The combined organic layers were washed with a saturated aqueous solution of $\mathrm{NaCl}$, dried over anhydrous $\mathrm{Na}_{2} \mathrm{SO}_{4}$ and evaporated under reduced pressure to give a white solid. This crude product was recrystallized from cyclohexane to afford substrates $\mathbf{4}$ as white crystals. 


\section{(C) General Procedure for the Pd-catalyed Tandem Reaction.}

The products $2 \mathrm{a}-2 \mathrm{q}$ were prepared according to Scheme S4:

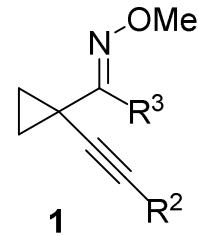

$\underset{\mathrm{AcOH}, \mathrm{rt}, 1 \mathrm{~h}-8 \mathrm{~h}}{\stackrel{\mathrm{Pd}(\mathrm{TFA})_{2}(2.5 \mathrm{~mol} \%)}{\longrightarrow}}$<smiles>[R]c1cc(CCOC(C)=O)c([R])n1OC</smiles>

Scheme S4

To a dried $25 \mathrm{~mL}$ Schlenk tube was added Pd(TFA) $)_{2}(2.5 \mathrm{~mol} \%)$, then the tube was evacuated and backfilled with Ar for 3 times. Compound 1 ( $0.2 \mathrm{mmol}, 1.0$ equiv), $2.0 \mathrm{~mL}$ AcOH were added under Ar protected and the resulting mixtures were stirred at room temperature. After the reaction was

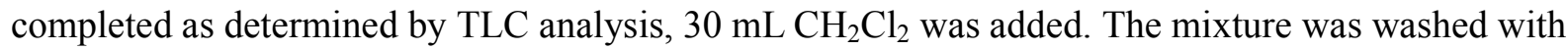
$\mathrm{H}_{2} \mathrm{O}(20 \mathrm{~mL})$, saturated sodium carbonate solution $(20 \mathrm{~mL})$. The organic layer was dried over anhydrous $\mathrm{Na}_{2} \mathrm{SO}_{4}$ and evaporated under reduced pressure. The residue was purified by column chromatography on silica gel (eluent: petroleum ether/ethyl acetate $=10 / 1$ ) afforded the corresponding products $\mathbf{2 a - 2 q}$.

Table S1. Optimization of the Solvents ${ }^{a, c}$

\begin{tabular}{|c|c|c|c|c|}
\hline entry & cat. (mol \%) & solvent & $\mathrm{t}(\mathrm{h})$ & yield $(\%)^{b}$ \\
\hline 1 & $\operatorname{Pd}(\mathrm{TFA})_{2}(5)$ & $\mathrm{CH}_{2} \mathrm{Cl}_{2}$ & 24 & 62 \\
\hline 2 & $\operatorname{Pd}(\mathrm{TFA})_{2}(5)$ & DCE & 24 & 58 \\
\hline 3 & $\operatorname{Pd}(\mathrm{TFA})_{2}(5)$ & THF & 24 & 42 \\
\hline 4 & $\operatorname{Pd}(\mathrm{TFA})_{2}(5)$ & $\mathrm{Et}_{2} \mathrm{O}$ & 24 & 38 \\
\hline 5 & $\operatorname{Pd}(\mathrm{TFA})_{2}(5)$ & DMF & 24 & trace \\
\hline
\end{tabular}

${ }^{a}$ The reaction conditions: $0.1 \mathrm{M}$ in solvent unless otherwise specified. ${ }^{b}$ The yield was determined by ${ }^{1} \mathrm{H}$ NMR spectroscopic data using 1,3,5-trimethoxybenzene as an internal standard. ${ }^{c}$ With 5.0 equiv $\mathrm{AcOH}$.

Table S2. Optimization of the Reaction Conditions ${ }^{a}$ 


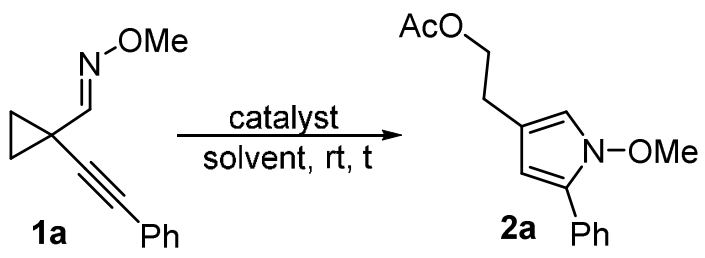

\begin{tabular}{ccccc}
\hline entry & cat. $(\mathrm{mol} \%)$ & solvent & $\mathrm{t}(\mathrm{h})$ & yield (\%) \\
\hline $\mathbf{1}$ & $\mathrm{Pd}(\mathrm{TFA})_{2}(5)$ & $\mathrm{AcOH}$ & 0.5 & 52 \\
$\mathbf{2}$ & $\mathrm{Pd}(\mathrm{TFA})_{2}(5)$ & $\mathrm{AcOH}$ & 1 & 93 \\
$\mathbf{3}$ & $\mathrm{Pd}(\mathrm{TFA})_{2}(5)$ & $\mathrm{AcOH}$ & 2 & 94 \\
$\mathbf{4}$ & $\mathrm{Pd}(\mathrm{TFA})_{2}(5)$ & $\mathrm{AcOH}$ & 12 & 92 \\
$\mathbf{5}^{c}$ & $\mathrm{Pd}(\mathrm{TFA})_{2}(5)$ & $\mathrm{AcOH}$ & 2 & 86 \\
$\mathbf{6}^{d}$ & $\mathrm{Pd}^{2}(\mathrm{TFA})_{2}(5)$ & $\mathrm{AcOH}$ & 2 & 88 \\
$\mathbf{7}^{e}$ & $\operatorname{Pd}(\mathrm{TFA})_{2}(5)$ & $\mathrm{AcOH}$ & 2 & 76 \\
$\mathbf{8}$ & $\mathrm{Fe}(\mathrm{OTf})_{3}(5)$ & $\mathrm{AcOH}$ & 24 & 0 \\
$\mathbf{9}$ & $\mathrm{Cu}(\mathrm{OTf})_{2}(5)$ & $\mathrm{AcOH}$ & 24 & 0 \\
\hline
\end{tabular}

${ }^{a}$ The reaction conditions: $0.1 \mathrm{M}$ in solvent unless otherwise specified. ${ }^{b}$ The yield was determined by ${ }^{1} \mathrm{H}$ NMR spectroscopic data using 1,3,5-trimethoxybenzene as an internal standard. ${ }^{c}$ With untreated AcOH. ${ }^{d}$ Without Ar protection. ${ }^{e}$ With 1.0 equiv $\mathrm{H}_{2} \mathrm{O}$.

The products 3a-3g were prepared according to Scheme S5:
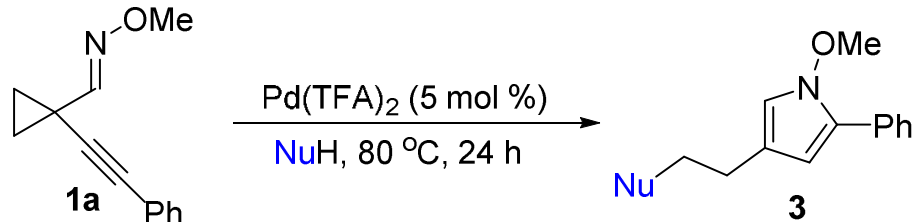

Scheme S5

To a dried $25 \mathrm{~mL}$ Schlenk tube was added Pd(TFA) $)_{2}(5 \mathrm{~mol} \%)$, then the reaction tube was evacuated and backfilled with Ar for 3 times. Nucleophiles ( 0.8 mmol, 2 equiv), compound $1 \mathrm{a}$ ( 0.4 mmol, 1.0 equiv) and $0.4 \mathrm{~mL}$ DCM were added under Ar atmosphere and the resulting mixtures were stirred at $80^{\circ} \mathrm{C}$. After the reaction was complete as monitored by TLC analysis, $30 \mathrm{~mL} \mathrm{CH}_{2} \mathrm{Cl}_{2}$ was added. The mixture was washed with saturated sodium chloride solution $(20 \mathrm{~mL})$. The organic layer was dried over anhydrous $\mathrm{Na}_{2} \mathrm{SO}_{4}$ and evaporated under reduced pressure. The residue was purified by column chromatography on silica gel (eluent: petroleum ether/ethyl acetate $=10 / 1$ ) to 
afford the corresponding products $\mathbf{3 a - 3 g}$.

The products 5a-5h were prepared according to Scheme S6:
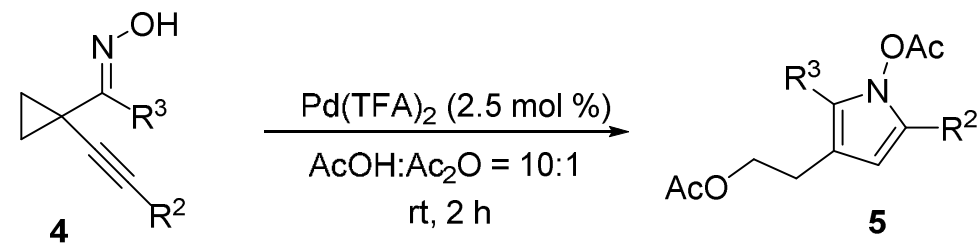

Scheme S6

To a dried $25 \mathrm{~mL}$ Schlenk tube was added Pd(TFA) $2(2.5 \mathrm{~mol} \%)$, then the reaction tube was evacuated and backfilled with $\mathrm{Ar}$ for 3 times. $2.0 \mathrm{~mL} \mathrm{AcOH}$ and $0.2 \mathrm{~mL} \mathrm{Ac} \mathrm{A}_{2} \mathrm{O}$ were added under $\mathrm{Ar}$ atmosphere and the resulting mixtures were stirred at room temperature. The compound 4 (0.2 mmol, 1.0 equiv) was added dropwise within $1 \mathrm{~h}$. After the reaction was complete as monitored by TLC analysis, $30 \mathrm{~mL} \mathrm{CH}_{2} \mathrm{Cl}_{2}$ was added. The mixture was washed with $\mathrm{H}_{2} \mathrm{O}(20 \mathrm{~mL})$ and saturated sodium carbonate solution $(20 \mathrm{~mL})$. The organic layer was dried over anhydrous $\mathrm{Na}_{2} \mathrm{SO}_{4}$ and evaporated under reduced pressure. The residue was purified by column chromatography on silica gel (eluent: petroleum ether/ethyl acetate $=10 / 1$ ) to afford the corresponding products $\mathbf{5 a}-\mathbf{5} \mathbf{h}$.

Table S3. Optimization of the Reaction Conditions ${ }^{a, b}$

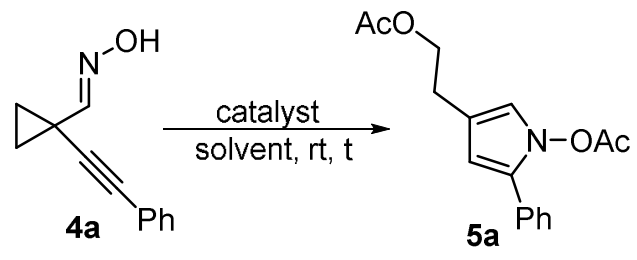

\begin{tabular}{|c|c|c|c|c|}
\hline entry & cat. (mol \%) & solvent & $\mathrm{t}(\mathrm{h})$ & yield $(\%)^{c}$ \\
\hline 1 & $\operatorname{Pd}(\mathrm{TFA})_{2}(5)$ & $\mathrm{AcOH}$ & 2 & 10 \\
\hline 2 & $\operatorname{Pd}(\mathrm{TFA})_{2}(5)$ & $\mathrm{AcOH} / \mathrm{Ac}_{2} \mathrm{O}$ & 2 & 48 \\
\hline 3 & $\mathrm{Pd}(\mathrm{OAc})_{2}(5)$ & $\mathrm{AcOH} / \mathrm{Ac}_{2} \mathrm{O}$ & 2 & 28 \\
\hline 4 & $\mathrm{Pd}(\mathrm{OTf})_{2}(5)$ & $\mathrm{AcOH} / \mathrm{Ac}_{2} \mathrm{O}$ & 2 & 51 \\
\hline 5 & $\mathrm{Pd}_{2}(\mathrm{dba})_{3}(5)$ & $\mathrm{AcOH} / \mathrm{Ac}_{2} \mathrm{O}$ & 24 & 45 \\
\hline 6 & $\mathrm{Pd}\left(\mathrm{PPh}_{3}\right)_{4}(5)$ & $\mathrm{AcOH} / \mathrm{Ac}_{2} \mathrm{O}$ & 24 & 36 \\
\hline 7 & $\operatorname{Pd}(\mathrm{TFA})_{2}(2.5)$ & $\mathrm{AcOH} / \mathrm{Ac}_{2} \mathrm{O}$ & 2 & 48 \\
\hline
\end{tabular}




$\begin{array}{cllcc}\mathbf{8} & \mathrm{Pd}(\mathrm{TFA})_{2}(10) & \mathrm{AcOH} / \mathrm{Ac}_{2} \mathrm{O} & 2 & 49 \\ \mathbf{9}^{d} & \mathrm{Pd}(\mathrm{TFA})_{2}(5) & \mathrm{AcOH} / \mathrm{Ac}_{2} \mathrm{O} & 2 & 32 \\ \mathbf{1 0}^{e} & \mathrm{Pd}(\mathrm{TFA})_{2}(5) & \mathrm{DCM} / \mathrm{Ac}_{2} \mathrm{O} & 2 & \operatorname{trace} \\ \mathbf{1 1}^{f} & \mathrm{Pd}(\mathrm{TFA})_{2}(5) & \mathrm{AcOH} / \mathrm{Ac}_{2} \mathrm{O} & 2 & 65 \\ \mathbf{1 2}^{f} & \mathrm{Pd}(\mathrm{TFA})_{2}(2.5) & \mathrm{AcOH} / \mathrm{Ac}_{2} \mathrm{O} & 2 & 66(65)^{g} \\ \mathbf{1 3} & - & \mathrm{AcOH} / \mathrm{Ac}_{2} \mathrm{O} & 24 & 0 \\ \mathbf{1 4} & \mathrm{Fe}(\mathrm{OTf})_{3}(5) & \mathrm{AcOH} / \mathrm{Ac} c_{2} \mathrm{O} & 24 & 0 \\ \mathbf{1 5} & \mathrm{Sc}(\mathrm{OTf})_{3}(5) & \mathrm{AcOH} / \mathrm{Ac}_{2} \mathrm{O} & 24 & 0 \\ \mathbf{1 6} & \left\{\mathrm{Rh}(\mathrm{COD}) \mathrm{Cl}_{2}\right\}_{2}(2.5) & \mathrm{AcOH} / \mathrm{Ac}_{2} \mathrm{O} & 24 & \text { trace } \\ \mathbf{1 7} & \mathrm{Ru}(\mathrm{CO})_{12}(5) & \mathrm{AcOH} / \mathrm{Ac}_{2} \mathrm{O} & 24 & \text { trace } \\ \mathbf{1 8} & \mathrm{Pt}(\mathrm{COD}) \mathrm{Cl}_{2}(5) & \mathrm{AcOH} / \mathrm{Ac}_{2} \mathrm{O} & 24 & 34 \\ \mathbf{1 9} & \mathrm{PPh}_{3} \mathrm{AuOTf}(5) & \mathrm{AcOH} / \mathrm{Ac}_{2} \mathrm{O} & 24 & 48\end{array}$

${ }^{a}$ The reaction conditions: $0.1 \mathrm{M}$ in solvent unless otherwise specified. ${ }^{b} \mathrm{The}$ solvent $\mathrm{AcOH} / \mathrm{Ac}_{2} \mathrm{O}=$ 10:1 unless otherwise specified. ${ }^{c}$ The yield was determined by ${ }^{1} \mathrm{H}$ NMR spectroscopic data using 1,3,5-trimethoxybenzene as an internal standard. ${ }^{d}$ With $\mathrm{AcOH} / \mathrm{Ac}_{2} \mathrm{O}=1: 1 .{ }^{e} \mathrm{With}^{\mathrm{DCM}} / \mathrm{Ac}_{2} \mathrm{O}=$ 10:1. ${ }^{f}$ The substrate was added dropwise in $1 \mathrm{~h} .{ }^{g}$ Isolated yield was provided.

The products 6a-6e were prepared according to Scheme S7:
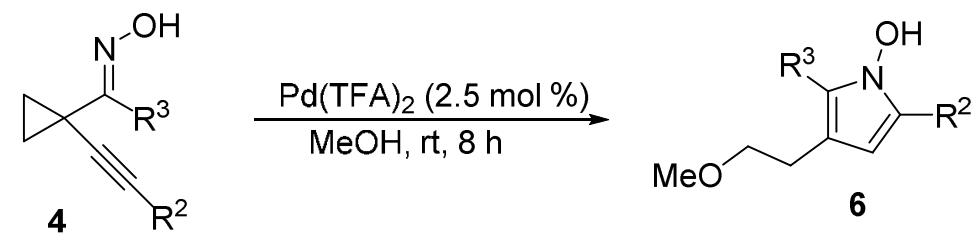

\section{Scheme S7}

To a dried $25 \mathrm{~mL}$ Schlenk tube was added Pd(TFA) 2 (2.5 mol \%), then the tube was evacuated and backfilled with Ar for 3 times. Compound 4 (0.2 mmol, 1.0 equiv) and $2.0 \mathrm{~mL} \mathrm{MeOH}$ were added under Ar atmosphere. After the reaction mixture was stirred at room temperature for $8 \mathrm{~h}$, the solvent was evaporated under reduced pressure while the temperature was kept lower than $30{ }^{\circ} \mathrm{C}$ because the products were not very stable. The residue was purified rapidly by a column chromatography on silica gel (eluent: petroleum ether/ethyl acetate $=10 / 1)$ to afford the corresponding products $\mathbf{6 a - 6 e}$. 


\section{(D) The Control Experiments}

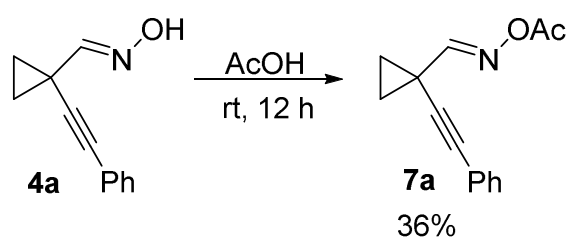

Scheme S8

To a dried $25 \mathrm{~mL}$ Schlenk tube was added compound $4 \mathbf{a}(0.2 \mathrm{mmol}, 1.0$ equiv), then the reaction tube was evacuated and backfilled with Ar for 3 times. $2.0 \mathrm{~mL} \mathrm{AcOH}$ was added under $\mathrm{Ar}$ atmosphere and the resulting mixtures were stirred at room temperature. After $12 \mathrm{~h}, 30 \mathrm{~mL} \mathrm{CH}_{2} \mathrm{Cl}_{2}$ was added. The mixture was washed with $\mathrm{H}_{2} \mathrm{O}(20 \mathrm{~mL})$ and saturated sodium carbonate solution $(20 \mathrm{~mL})$. The organic layer was dried over anhydrous $\mathrm{Na}_{2} \mathrm{SO}_{4}$ and evaporated under reduced pressure. The residue was purified by column chromatography on silica gel (eluent: petroleum ether/ethyl acetate $=20 / 1)$ to afford $7 \mathbf{a}$ in $36 \%$ yield (Scheme S8).

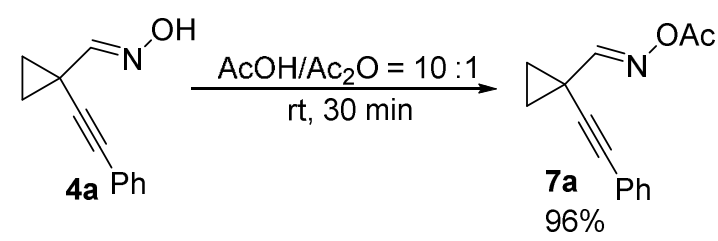

Scheme S9

To a dried $25 \mathrm{~mL}$ Schlenk tube was added compound $4 \mathrm{a}(0.2 \mathrm{mmol}, 1.0$ equiv), then the tube was evacuated and backfilled with Ar for 3 times. $2.0 \mathrm{~mL} \mathrm{AcOH}$ and $0.2 \mathrm{~mL} \mathrm{Ac} \mathrm{A}_{2} \mathrm{O}$ were added under $\mathrm{Ar}$ atmosphere and the resulting mixtures were stirred at room temperature. After $30 \mathrm{~min}, 30 \mathrm{~mL}$ $\mathrm{CH}_{2} \mathrm{Cl}_{2}$ was added. The mixture was washed with $\mathrm{H}_{2} \mathrm{O}(20 \mathrm{~mL})$ and saturated sodium carbonate solution $(20 \mathrm{~mL})$. The organic layer was dried over anhydrous $\mathrm{Na}_{2} \mathrm{SO}_{4}$ and evaporated under reduced pressure. The residue was purified by column chromatography on silica gel (eluent: petroleum ether/ethyl acetate $=20 / 1)$ to afford $7 \mathbf{a}$ in $96 \%$ yield (Scheme S9).

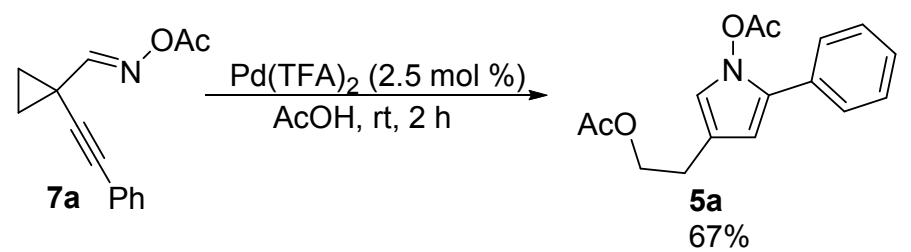




\section{Scheme S10}

To a dried $25 \mathrm{~mL}$ Schlenk tube was added Pd(TFA $)_{2}(2.5 \mathrm{~mol} \%)$, then the tube was evacuated and backfilled with Ar for 3 times. $2.0 \mathrm{~mL} \mathrm{AcOH}$ were added under Ar atmosphere and the resulting mixtures were stirred at room temperature. The compound $7 \mathbf{a}(0.2 \mathrm{mmol}, 1.0$ equiv) was added dropwise within $1 \mathrm{~h}$. After the reaction was complete as monitored by TLC analysis, $30 \mathrm{~mL} \mathrm{CH}_{2} \mathrm{Cl}_{2}$ was added. The mixture was washed with $\mathrm{H}_{2} \mathrm{O}(20 \mathrm{~mL})$ and saturated sodium carbonate solution $(20 \mathrm{~mL})$. The organic layer was dried over anhydrous $\mathrm{Na}_{2} \mathrm{SO}_{4}$ and evaporated under reduced pressure. The residue was purified by column chromatography on silica gel (eluent: petroleum ether/ethyl acetate $=10 / 1)$ to afford the product 5a in 67\% yield (Scheme S10). 


\section{(E) The Transformation of Product}

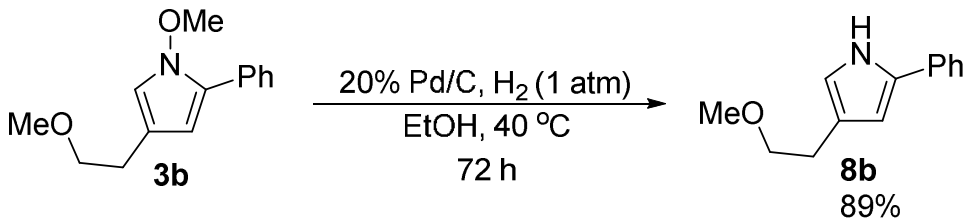

\section{Scheme S11}

To a dried $25 \mathrm{~mL}$ flask was added $20 \% \mathrm{Pd} / \mathrm{C}$, EtOH $10 \mathrm{~mL}$ and $\mathbf{3 b}(0.5 \mathrm{mmol})$, and then the reaction tube was evacuated and backfilled with $\mathrm{H}_{2}$ for 3 times. The resulting mixtures were stirred at $40{ }^{\circ} \mathrm{C}$ for $72 \mathrm{~h}$. The mixture was filtered through a Celite and washed with DCM $(10 \mathrm{~mL})$. The solvent was evaporated under reduced pressure and the residue was purified directly by a column chromatography on silica gel (eluent: petroleum ether/ethyl acetate $=4 / 1$ ) to afford the desired product $\mathbf{8 b}$ in $89 \%$ yield (Scheme S11). 


\section{(F) Deuterium Labeling Experiments}

\section{The deuterium labeling experiments of $4 a$}

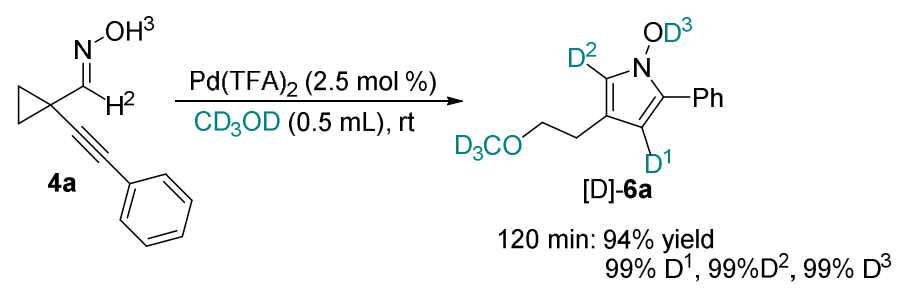

\section{Scheme S12}

Into a NMR tube, to a solution of compound $4 \mathrm{a}(0.1 \mathrm{mmol}, 1.0$ equiv) and internal standard 1,3,5-trimethoxybenzene $\left(0.1 \mathrm{mmol}, 1.0\right.$ equiv) in $\mathrm{CD}_{3} \mathrm{OD}(0.5 \mathrm{~mL})$ was added the catalyst $\operatorname{Pd}(\mathrm{TFA})_{2}(2.5 \mathrm{~mol} \%)$ at room temperature. Then the resulting solution was under ${ }^{1} \mathrm{H}-\mathrm{NMR}$ analysis. After 120 min, we can identify that the yield of [D]-6a was $94 \%$ along with $99 \% \mathrm{D}^{1}, 99 \%$ $\mathrm{D}^{2}$ and $99 \% \mathrm{D}^{3}$ incorporations (Scheme S12).

To identify the deuterated step of product, we carried out the followed control experiments:

(a) Into a NMR tube, compound $4 \mathbf{a}\left(0.1 \mathrm{mmol}, 1\right.$ equiv) and $\mathrm{CD}_{3} \mathrm{OD}(0.5 \mathrm{~mL})$ was added. Then the resulting solution was under ${ }^{1} \mathrm{H}-\mathrm{NMR}$ analysis at room temperature. After $120 \mathrm{~min}$, we could identify that the $\mathrm{H}^{3}$ was deuterated completely, but $\mathrm{H}^{2}$ could not be deuterated under the same condition.

(b) Into a NMR tube, compound $6 \mathbf{a}\left(0.1 \mathrm{mmol}, 1.0\right.$ equiv) and $\mathrm{CD}_{3} \mathrm{OD}(0.5 \mathrm{~mL})$ was added. Then the resulting solution was under ${ }^{1} \mathrm{H}-\mathrm{NMR}$ analysis at room temperature. After $120 \mathrm{~min}$, we could identify that the compound 6a was deuterated along with $15 \% \mathrm{D}^{1}, 75 \% \mathrm{D}^{2}$ and $99 \% \mathrm{D}^{3}$ incorporations.

(c) Into a NMR tube, compound 6a $\left(0.1 \mathrm{mmol}, 1\right.$ equiv), $\mathrm{Pd}(\mathrm{TFA})_{2}(2.5 \mathrm{~mol} \%)$ and $\mathrm{CD}_{3} \mathrm{OD}(0.5$ $\mathrm{mL}$ ) was added. Then the resulting solution was under ${ }^{1} \mathrm{H}-\mathrm{NMR}$ analysis at room temperature. After 120 min, we could identify that the $\mathrm{H}^{1}, \mathrm{H}^{2}$ and $\mathrm{H}^{3}$ were deuterated completely.

The ${ }^{1} \mathrm{H}$ NMR spectrum of the reaction mixture after $120 \mathrm{~min}$ : 

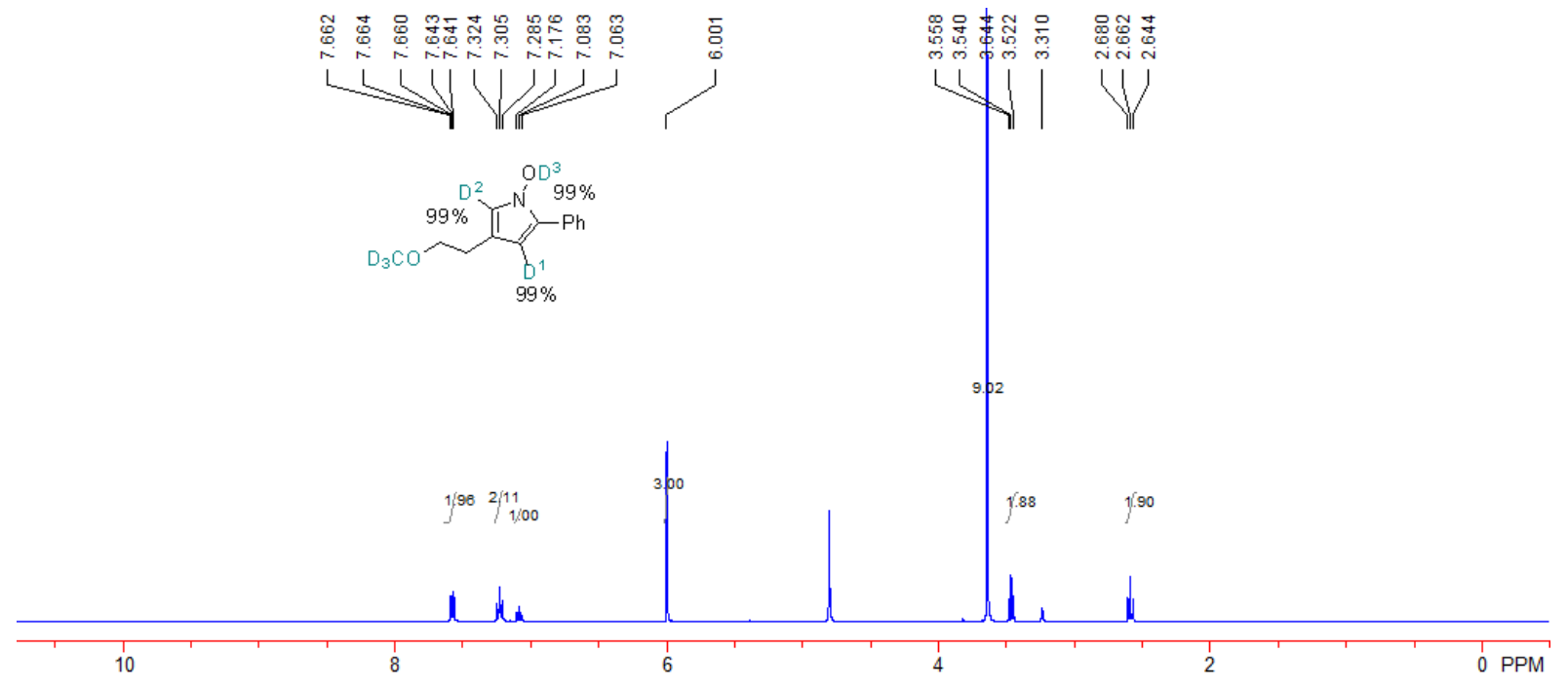

The ${ }^{1} \mathrm{H}$ NMR spectrum of $\mathbf{6 a}$ in $\mathrm{CDCl}_{3}$ :
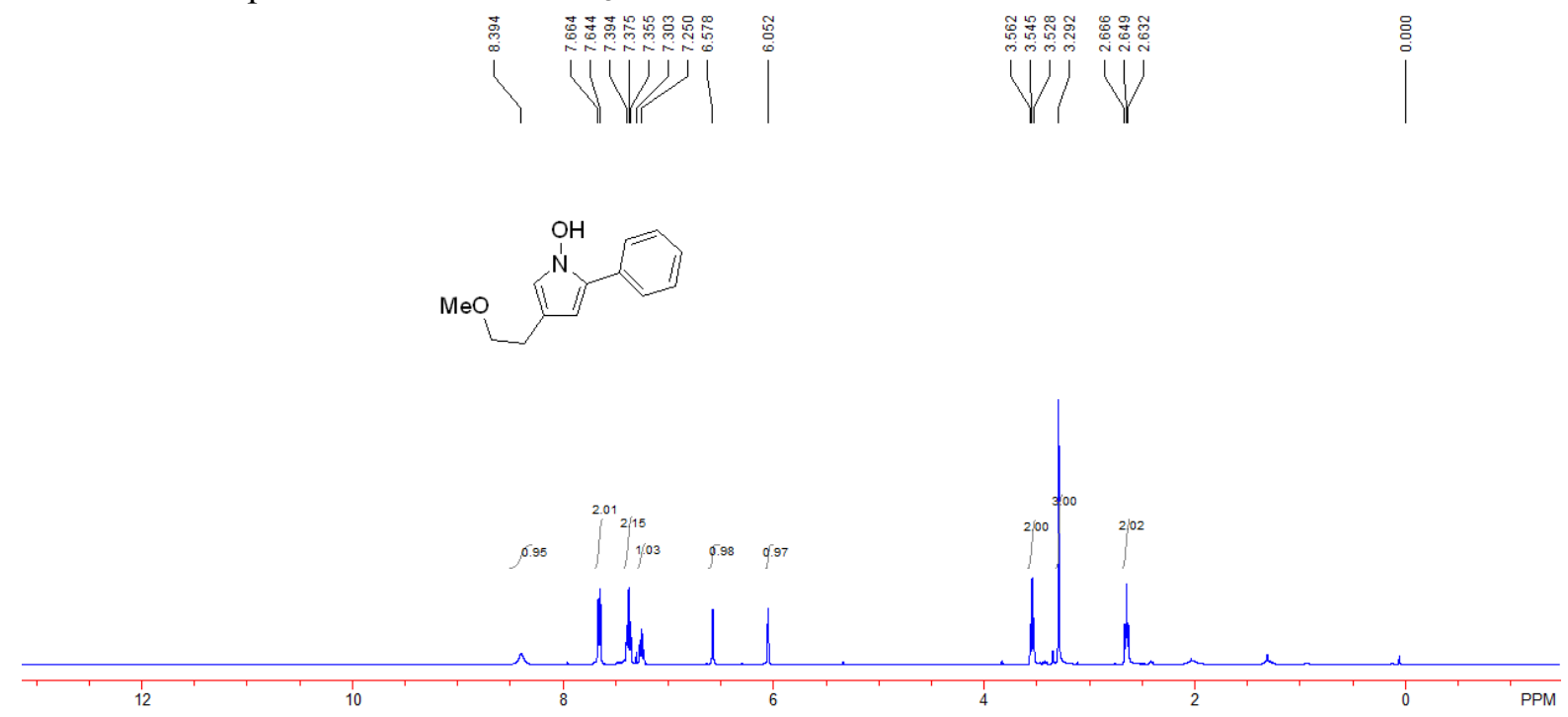

The ${ }^{1} \mathrm{H}$ NMR spectrum of $4 \mathbf{a}$ in $\mathrm{CD}_{3} \mathrm{OD}$ after $120 \mathrm{~min}$ :

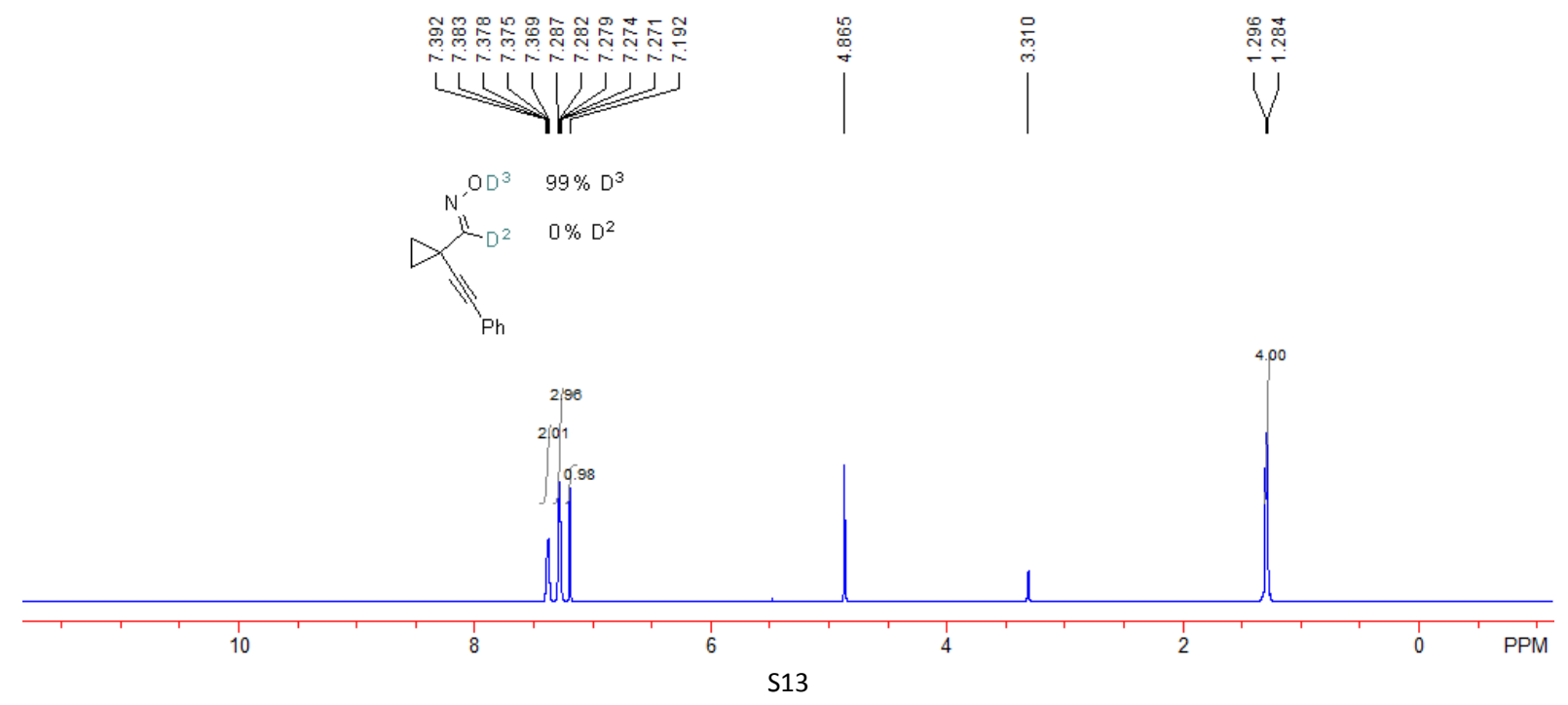


The ${ }^{1} \mathrm{H}$ NMR spectrum of $\mathbf{6 a}$ in $\mathrm{CD}_{3} \mathrm{OD}$ after $120 \mathrm{~min}$ :
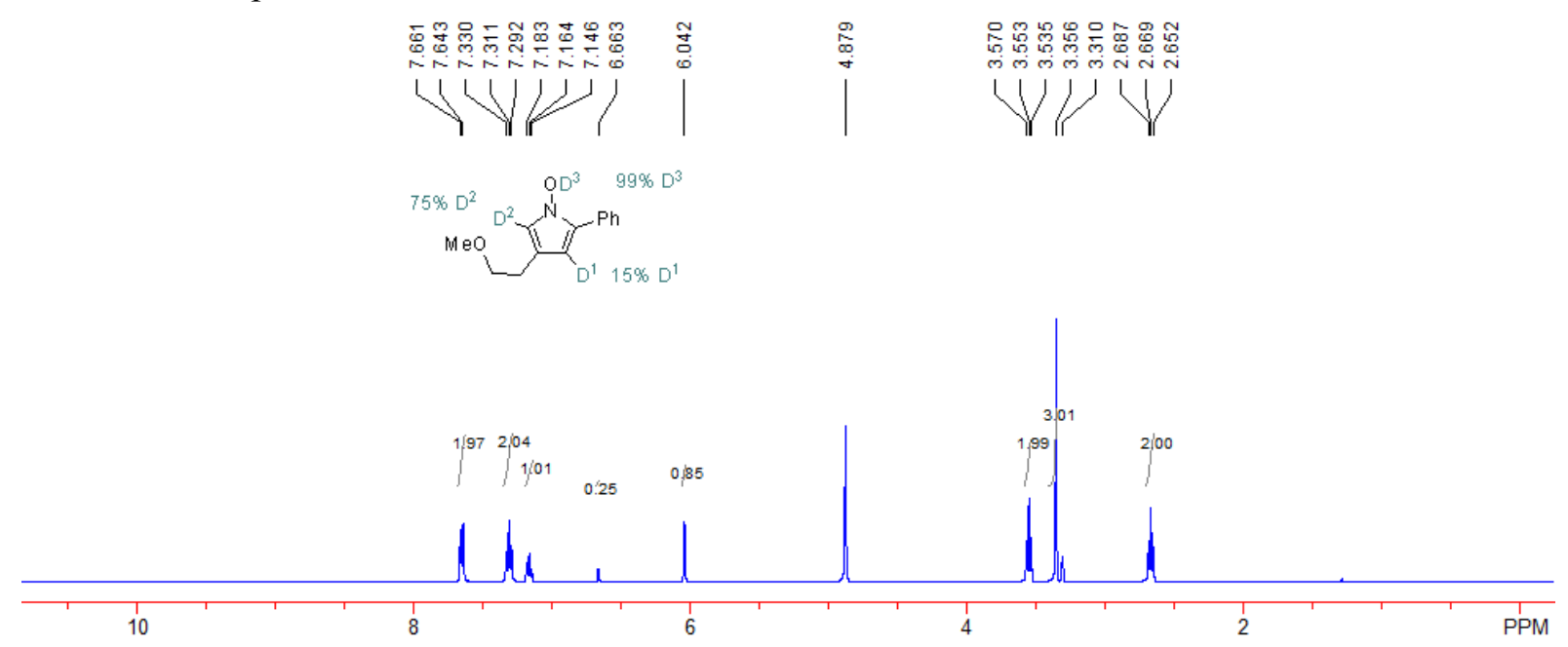

The ${ }^{1} \mathrm{H}$ NMR spectrum of 6 a with Pd(TFA) 2 in $\mathrm{CD}_{3} \mathrm{OD}$ after $120 \mathrm{~min}$ :
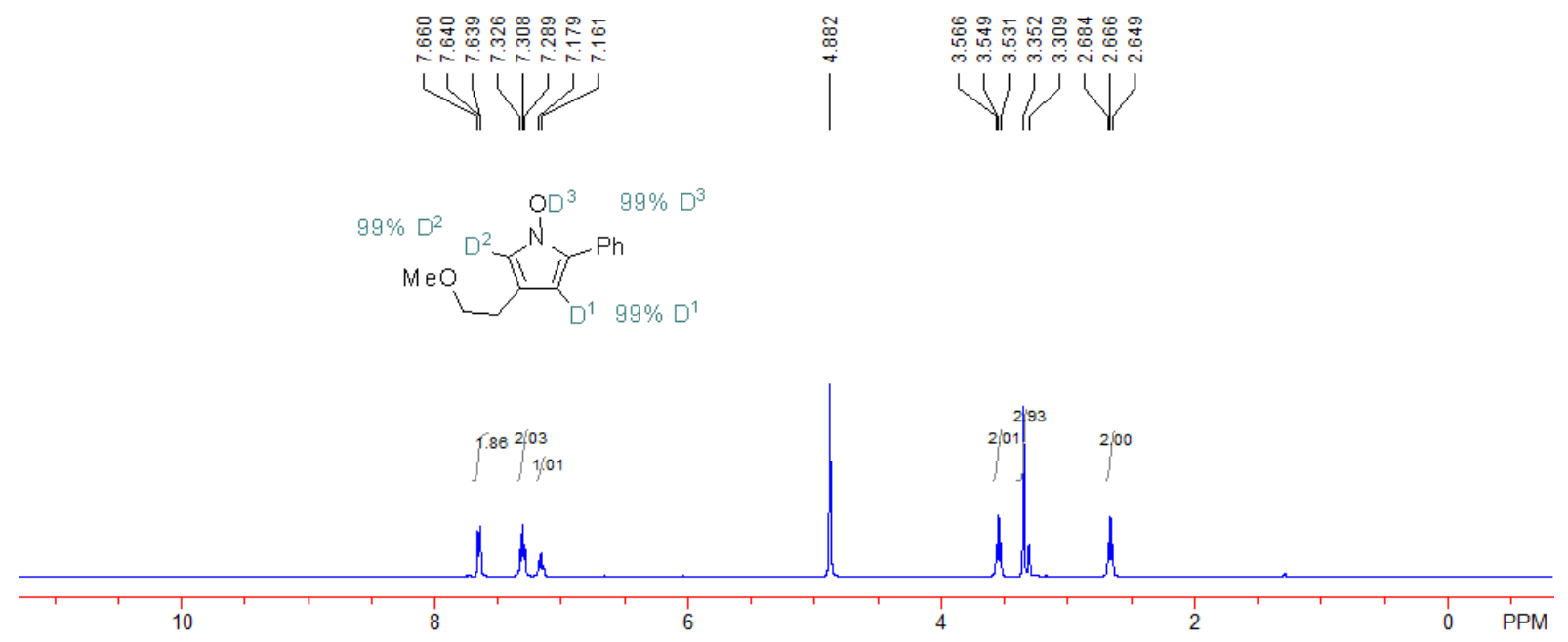

The deuterium labeling experiments of 1a

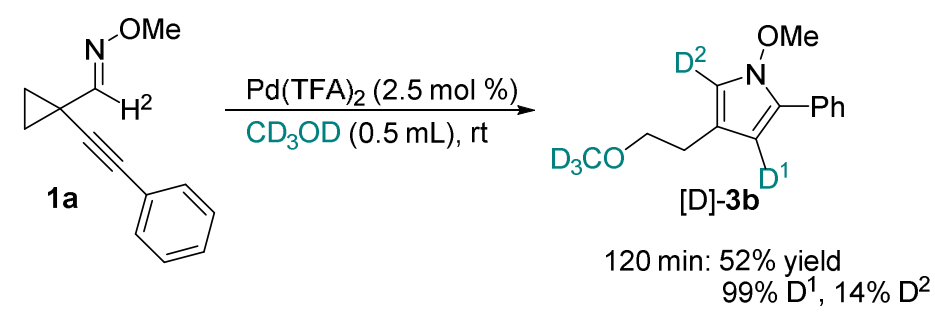

Scheme S13

Into a NMR tube, a solution of compound $1 \mathrm{a}(0.1 \mathrm{mmol}, 1$ equiv) and internal standard 1,3,5-trimethoxybenzene ( $0.1 \mathrm{mmol}, 1$ equiv) in $\mathrm{CD}_{3} \mathrm{OD}(0.5 \mathrm{~mL})$ was added the catalyst $\mathrm{Pd}(\mathrm{TFA})_{2}$ 
$(2.5 \mathrm{~mol} \%)$ at room temperature. Then the resulting solution was under ${ }^{1} \mathrm{H}-\mathrm{NMR}$ analysis. After 120 min, we could identify that the yield of [D]-3b was $52 \%$ along with $99 \% \mathrm{D}^{1}$ and $14 \% \mathrm{D}^{2}$ incorporations (Scheme S13).

To identify the deuterated step of product, we carried out the followed control experiments:

(a) Into a NMR tube, compound $1 \mathrm{a}\left(0.1 \mathrm{mmol}, 1.0\right.$ equiv) and $\mathrm{CD}_{3} \mathrm{OD}(0.5 \mathrm{~mL})$ was added. Then the resulting solution was under ${ }^{1} \mathrm{H}-\mathrm{NMR}$ analysis at room temperature. After $120 \mathrm{~min}$, we could identify that the $\mathrm{H}^{2}$ could not be deuterated under the same condition.

(b) Into a NMR tube, compound $\mathbf{3 b}\left(0.1 \mathrm{mmol}, 1.0\right.$ equiv) and $\mathrm{CD}_{3} \mathrm{OD}(0.5 \mathrm{~mL})$ was added. Then the resulting solution was under ${ }^{1} \mathrm{H}-\mathrm{NMR}$ analysis at room temperature. After $120 \mathrm{~min}$, we could identify that the $\mathrm{H}^{1}$ and $\mathrm{H}^{2}$ could not be deuterated under the condition.

(c) Into a NMR tube, compound $\mathbf{3 b}\left(0.1 \mathrm{mmol}, 1.0\right.$ equiv), $\mathrm{Pd}(\mathrm{TFA})_{2}(2.5 \mathrm{~mol} \%)$ and $\mathrm{CD}_{3} \mathrm{OD}(0.5$ $\mathrm{mL}$ ) was added. Then the resulting solution was under ${ }^{1} \mathrm{H}-\mathrm{NMR}$ analysis at room temperature. After $120 \mathrm{~min}$, we could identify that the compound was deuterated with $21 \% \mathrm{D}^{1}$ and $27 \% \mathrm{D}^{2}$ incorporations.

The ${ }^{1} \mathrm{H}$ NMR spectrum of the reaction mixture after $120 \mathrm{~min}$ :
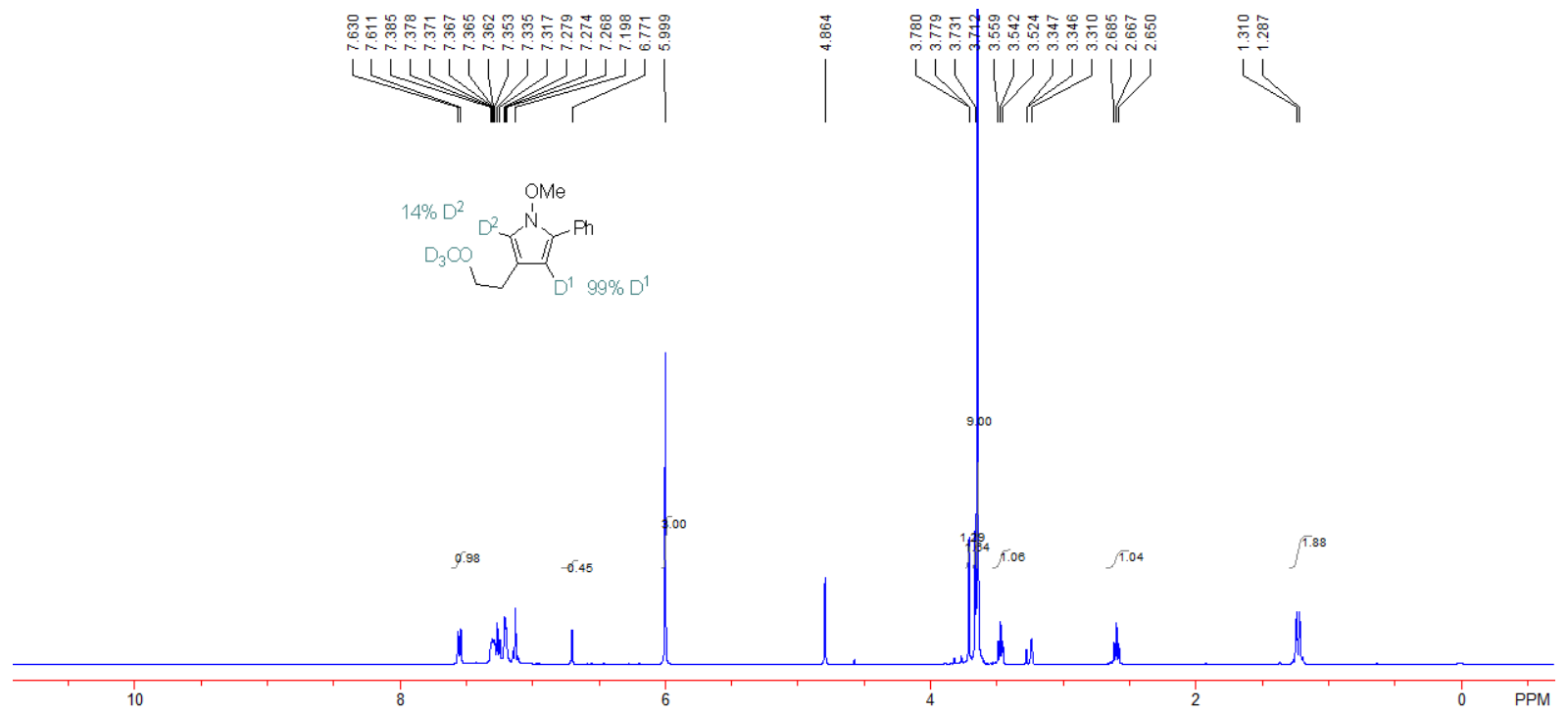

The ${ }^{1} \mathrm{H}$ NMR spectrum of $\mathbf{3 b}$ in $\mathrm{CDCl}_{3}$ : 


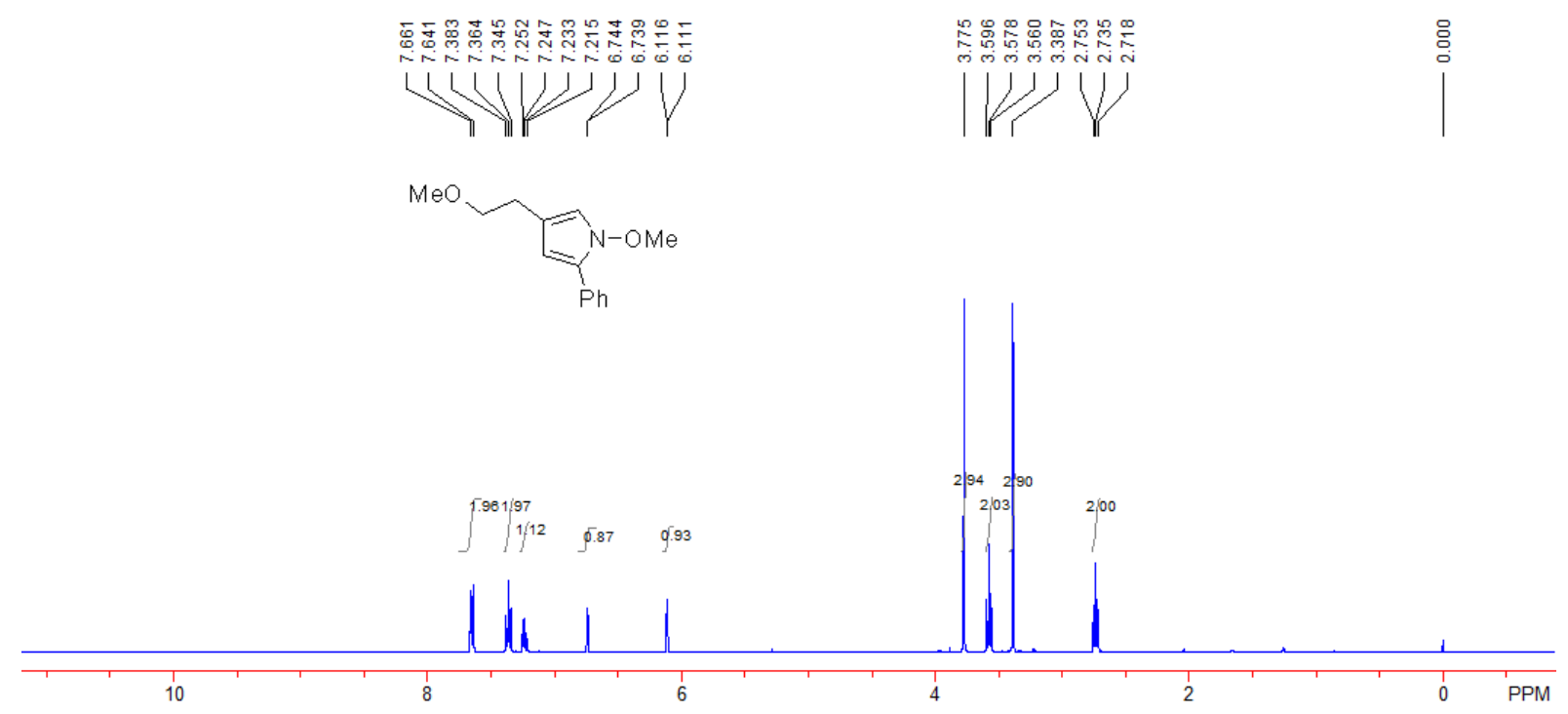

The ${ }^{1} \mathrm{H}$ NMR spectrum of $\mathbf{1 a}$ in $\mathrm{CD}_{3} \mathrm{OD}$ after $120 \mathrm{~min}$ :

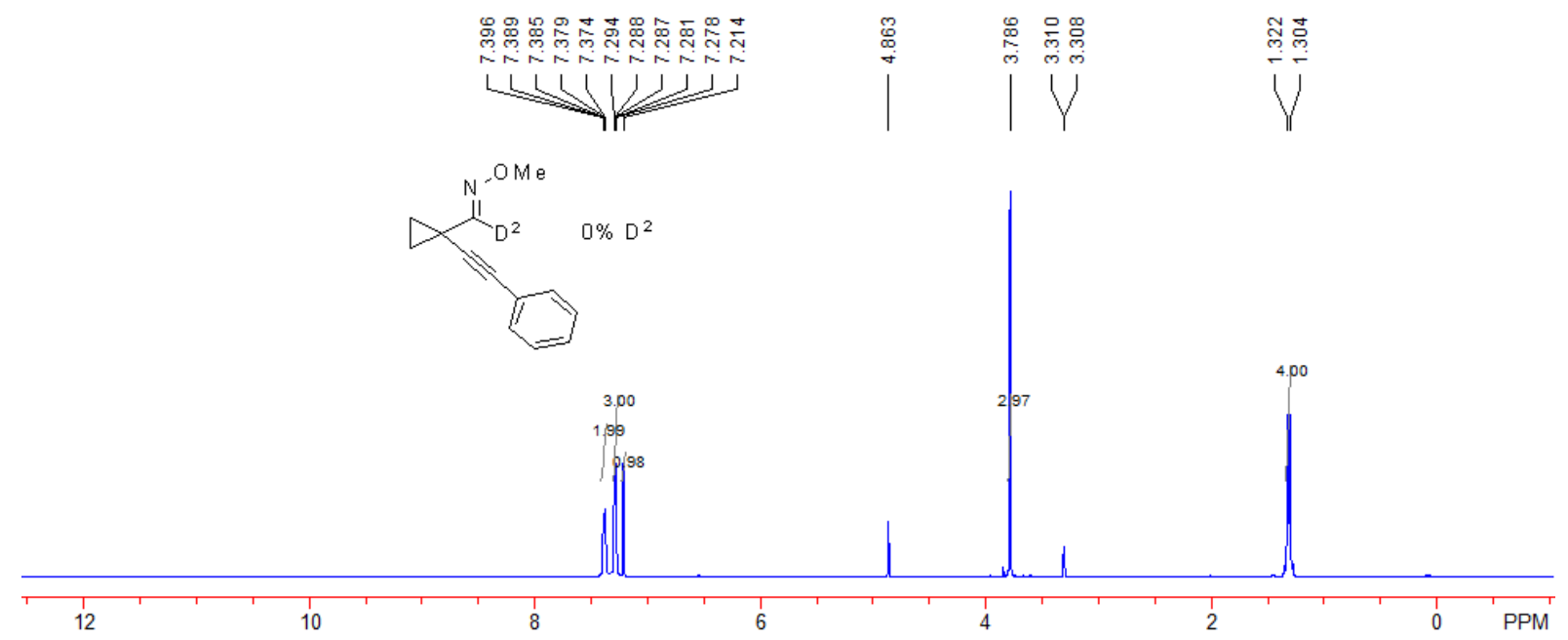

The ${ }^{1} \mathrm{H}$ NMR spectrum of $\mathbf{3 b}$ in $\mathrm{CD}_{3} \mathrm{OD}$ after 120 min:

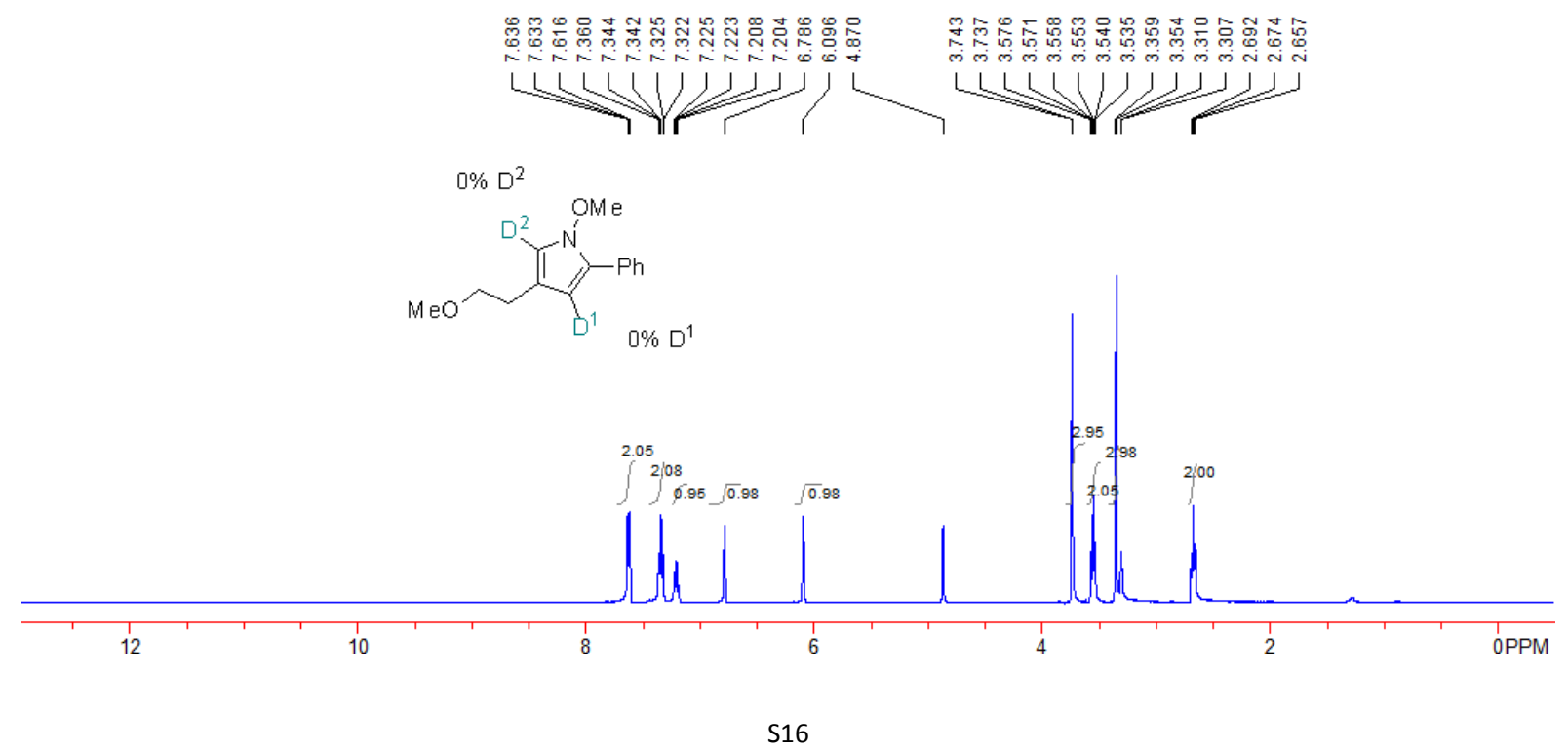


The ${ }^{1} \mathrm{H}$ NMR spectrum of $\mathbf{3 b}$ with $\mathrm{Pd}(\mathrm{TFA})_{2}$ in $\mathrm{CD}_{3} \mathrm{OD}$ after $120 \mathrm{~min}$ :
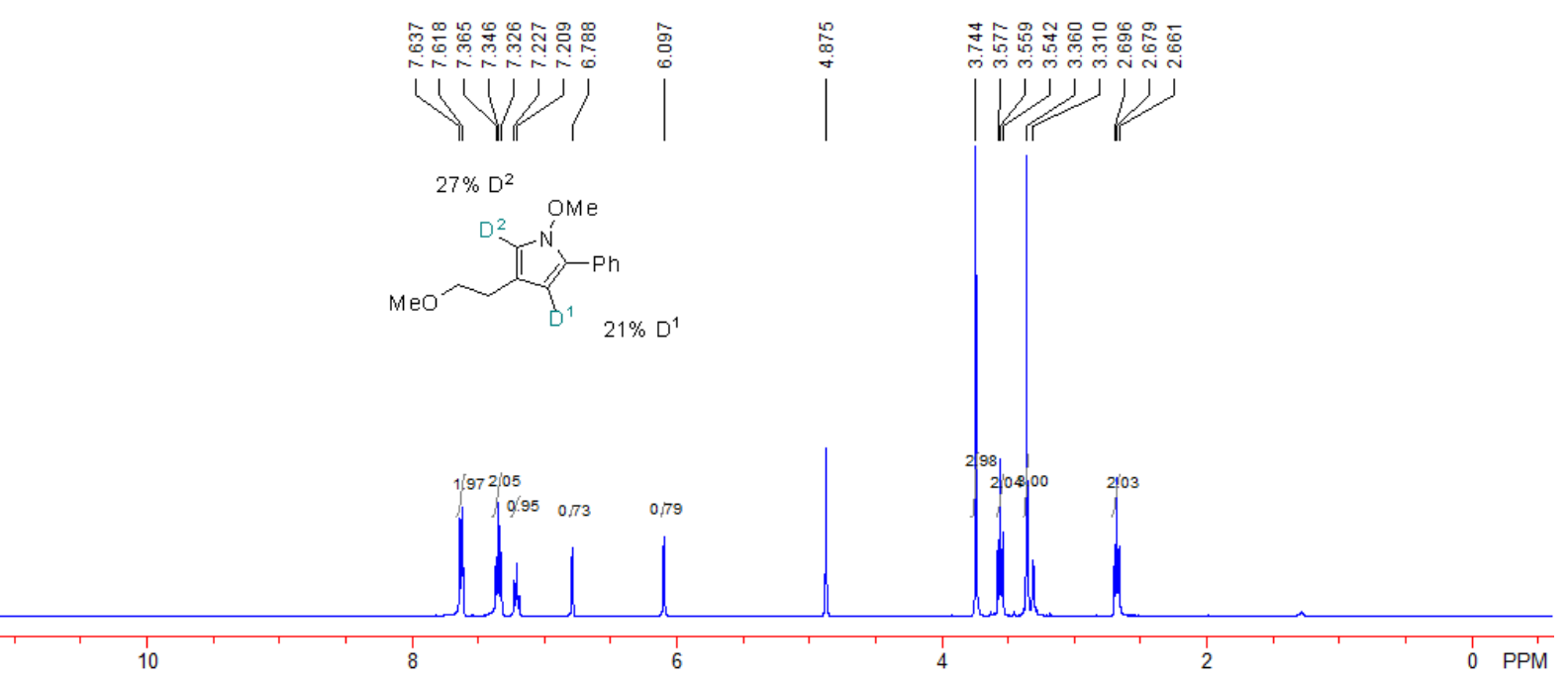

The proposed mechanism for the deuterated process

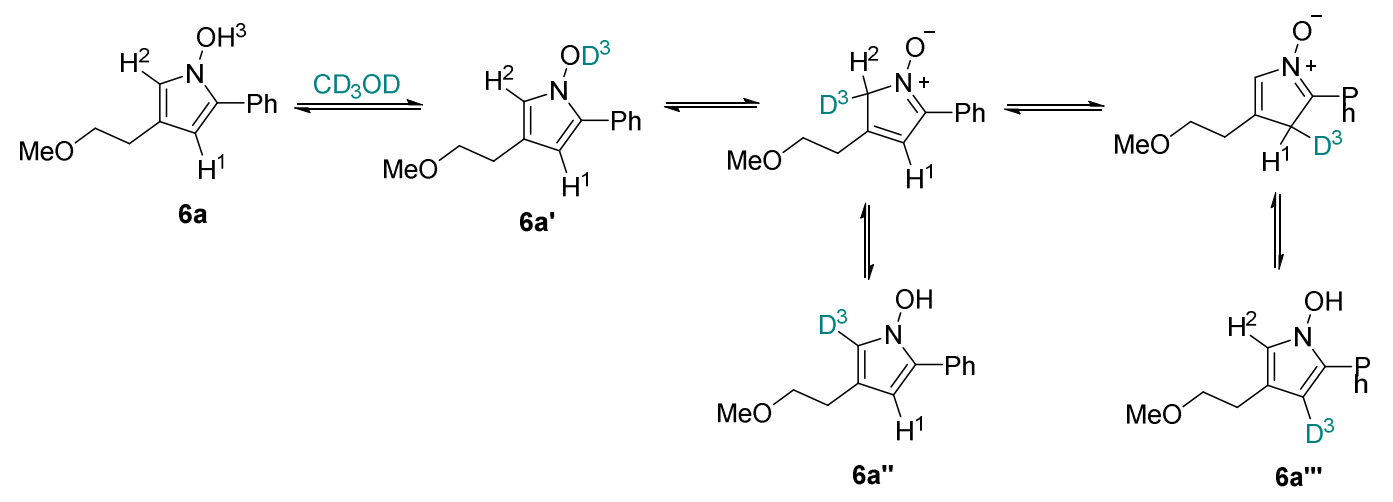

Scheme S14

The proposed mechanism for the formation of deuterated $6 \mathbf{a}$ was shown in Scheme $\mathrm{S} 14 .{ }^{5}$ The $\mathrm{H}^{3}$ of compound 6a could be deuterated rapidly by $\mathrm{CD}_{3} \mathrm{OD}$ through proton exchange to give 6a'. Then, the $\mathrm{H}^{2}$ deuterated compound $\mathbf{6 a}$ " and $\mathrm{H}^{1}$ deuterated compound $\mathbf{6 a}$ ", could be afforded by the tautomerization of compound 6a'. Of course, all of the protons in pyrrole ring could be deuterated finally. The addition of $\operatorname{Pd}(\mathrm{TFA})_{2}$ might be able to accelerate this process, and therefore, $\mathrm{H}^{1}, \mathrm{H}^{2}$ and $\mathrm{H}^{3}$ of product 6 a could be completely deuterated more quickly in $\mathrm{CD}_{3} \mathrm{OD}$ through the assistance of electrophilic palladation and the subsequent protonation. 

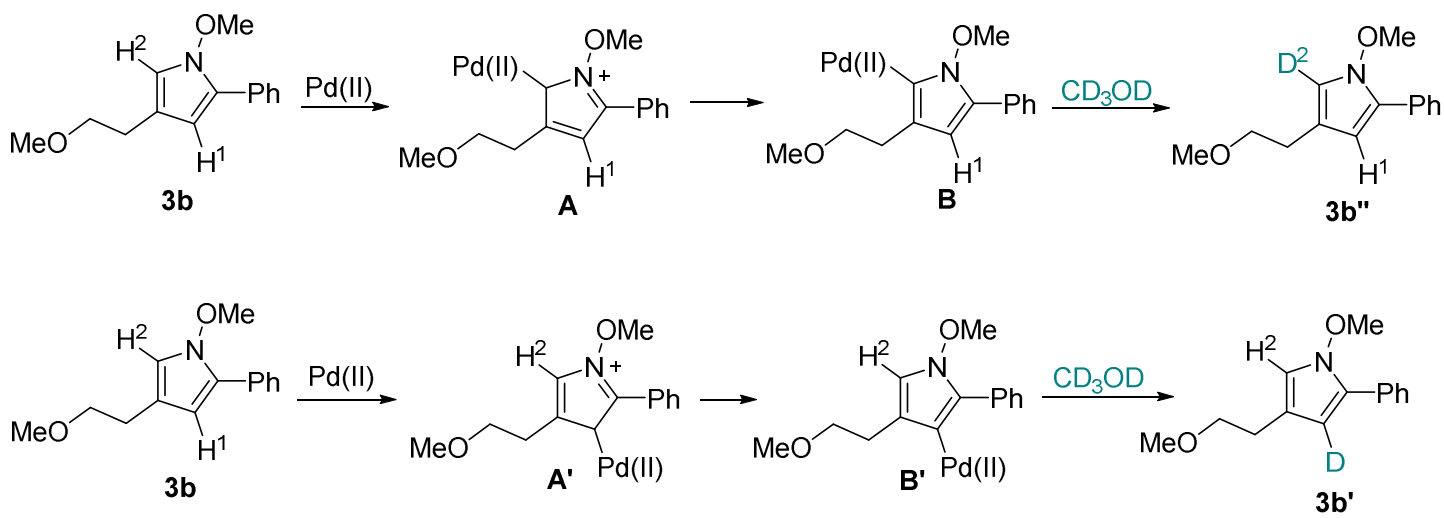

\section{Scheme S15}

The $\mathrm{H}^{1}$ and $\mathrm{H}^{2}$ of compound $\mathbf{3 b}$ could not be deuterated in $\mathrm{CD}_{3} \mathrm{OD}$. However, the deuterium incorporation took place in the presence of $\operatorname{Pd}(\mathrm{TFA})_{2}$, suggesting that $\operatorname{Pd}(\mathrm{TFA})_{2}$ played an important role in the deuterated process. A proposed mechanism for the deuteration of $\mathrm{H}^{1}$ and $\mathrm{H}^{2}$ of compound 3b was shown in Scheme S15. ${ }^{6}$ This process might involve electrophilic palladation of 3b to form the intermediates $\mathbf{A}$ and $\mathbf{A}^{\prime}$, followed by aromatization and protonation to afford the deuterated compounds $\mathbf{3} \mathbf{b}^{\text {'' }}$ and $\mathbf{3} \mathbf{b}$ '. 


\section{(G) Spectroscopic Data of Substrates and Products}

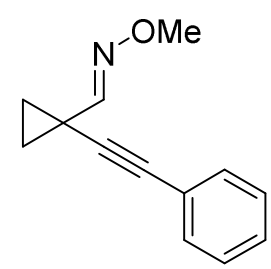

(E)-1-(phenylethynyl)cyclopropane-1-carbaldehyde O-methyl oxime 1a: Yield: $147 \mathrm{mg}, 74 \%$; A colorless oil; ${ }^{1} \mathrm{H}$ NMR $\left(\mathrm{CDCl}_{3}, 400 \mathrm{MHz}, \mathrm{TMS}\right) \delta 1.16-1.25\left(\mathrm{~m}, 4 \mathrm{H}, 2 \mathrm{CH}_{2}\right), 3.71\left(\mathrm{~s}, 3 \mathrm{H}, \mathrm{CH}_{3}\right)$, 7.13-7.15 (m, 3H, Ar), 7.18 (s, 1H, CH), 7.29-7.31 (m, 2H, Ar). $\left.{ }^{13} \mathrm{C} \mathrm{NMR} \mathrm{CDCl}_{3}, 100 \mathrm{MHz}, \mathrm{TMS}\right)$ $\delta 12.7,17.9,61.4,78.4,90.1,123.0,127.8,128.0,131.6,151.0 . \mathrm{IR}(\mathrm{EtOH}) \vee 3200,2938,2899$, $2816,2224,1597,1491,1464,1442,1305,1180,1059,1010,975,925,897,826,793,755,691$ $\mathrm{cm}^{-1}$. MS (ESI) m/z $200(\mathrm{M}+\mathrm{H})^{+}$. HRMS (ESI) calcd. for $\mathrm{C}_{13} \mathrm{H}_{14} \mathrm{NO}$ : 200.1070, Found: 200.1070.
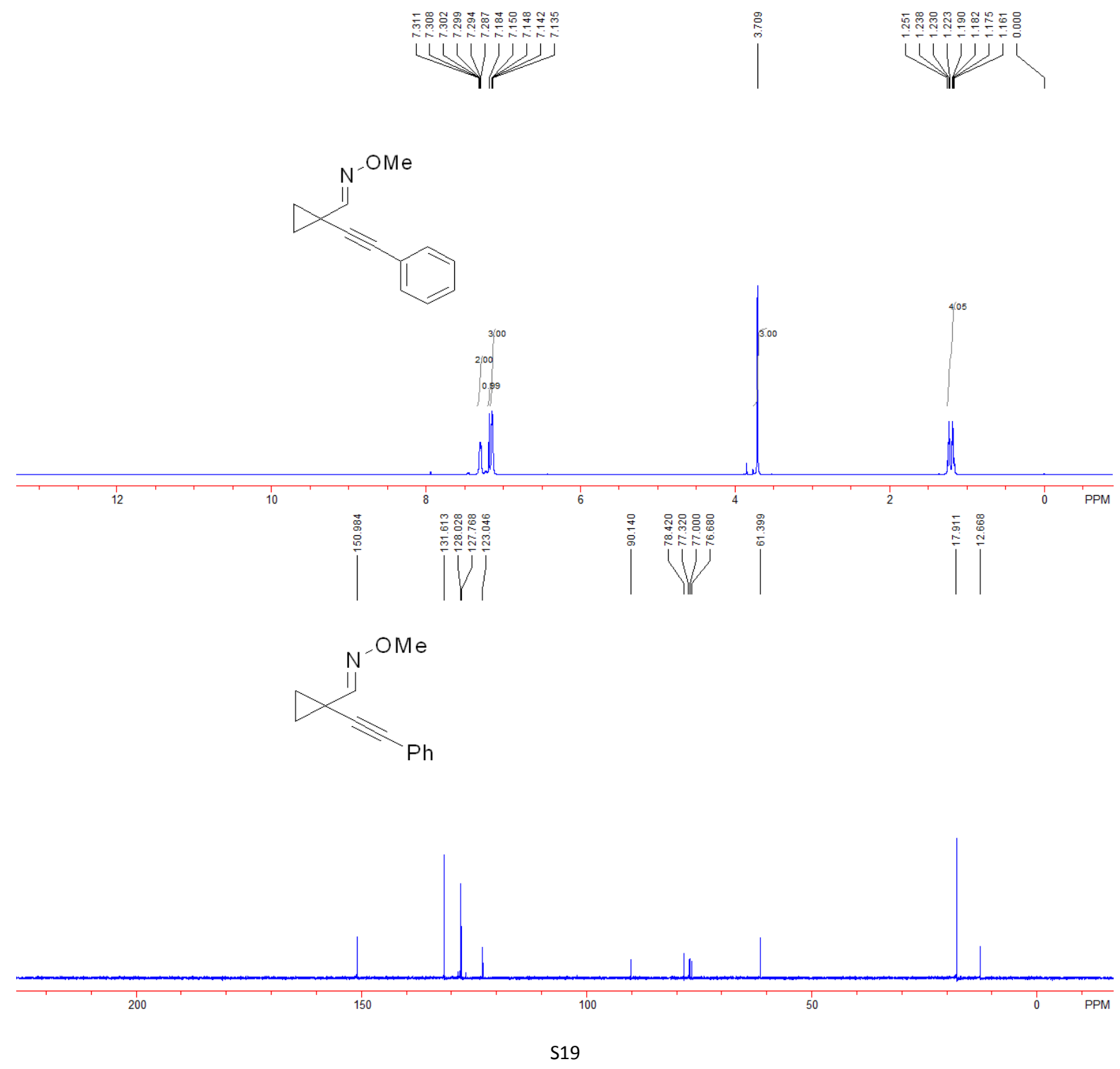


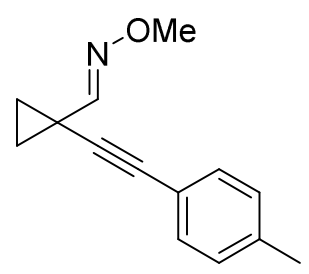

(E)-1-(p-tolylethynyl)cyclopropane-1-carbaldehyde O-methyl oxime 1b: Yield: $202 \mathrm{mg}$, 95\%; A colorless oil; ${ }^{1} \mathrm{H}$ NMR $\left(\mathrm{CDCl}_{3}, 400 \mathrm{MHz}, \mathrm{TMS}\right) \delta$ 1.28-1.37 (m, 4H, $\left.2 \mathrm{CH}_{2}\right), 2.33\left(\mathrm{~s}, 3 \mathrm{H}, \mathrm{CH}_{3}\right), 3.83$ (s, 3H, $\mathrm{CH}_{3}$ ), 7.07-7.09 (m, 2H, Ar), 7.26-7.31 (m, 3H, Ar, CH). ${ }^{13} \mathrm{C}$ NMR ( $\left.\mathrm{CDCl}_{3}, 100 \mathrm{MHz}, \mathrm{TMS}\right)$ $\delta 12.8,18.0,21.4,61.6,78.6,89.4,120.1,128.9,131.7,137.9,151.4$. IR (EtOH) $\vee 3056,2935$, 2815, 2226, 1677, 1597, 1491, 1443, 1343, 1285, 1203, 1178, 1066, 1034, 1014, 1001, 992, 951, 907, 841, 801, 771, 754, 709, 689, $669 \mathrm{~cm}^{-1}$. MS (ESI) m/z $214(\mathrm{M}+\mathrm{H})^{+}$. HRMS (ESI) calcd. for $\mathrm{C}_{14} \mathrm{H}_{16} \mathrm{NO}: 214.1226$, Found: 214.1227.

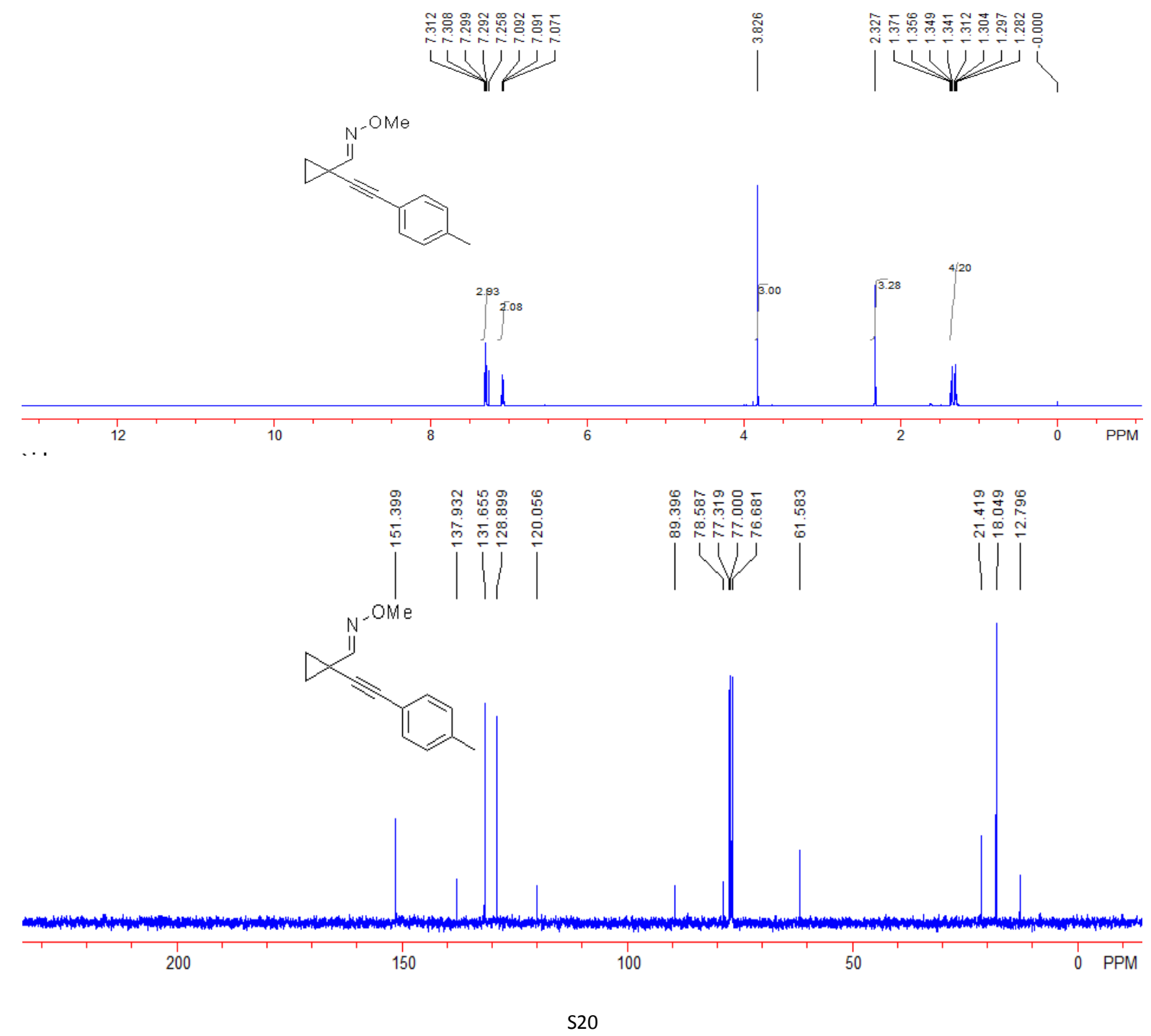




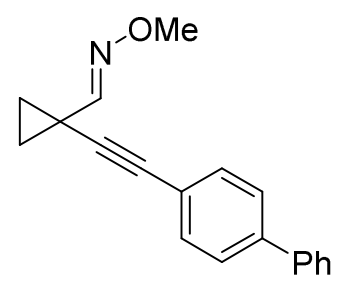

(E)-1-([1,1'-biphenyl]-4-ylethynyl)cyclopropane-1-carbaldehyde O-methyl oxime 1c: Yield: $253 \mathrm{mg}$, 92\%; A colorless oil; ${ }^{1} \mathrm{H}$ NMR $\left(\mathrm{CDCl}_{3}, 400 \mathrm{MHz}, \mathrm{TMS}\right) \delta 1.29-1.38\left(\mathrm{~m}, 4 \mathrm{H}, 2 \mathrm{CH}_{2}\right), 3.82$ (s, 3H, $\mathrm{CH}_{3}$ ), 7.29-7.33 (m, 2H, Ar), 7.38-7.42 (m, 2H, Ar), 7.45-7.50 (m, 3H, Ar, CH), 7.53-7.55 (m, 2H, Ar). ${ }^{13} \mathrm{C} \mathrm{NMR}\left(\mathrm{CDCl}_{3}, 100 \mathrm{MHz}, \mathrm{TMS}\right) \delta 12.8,18.1,61.5,78.4,90.9,122.1,126.8,126.9,127.5$, 128.7, 132.1, 140.5, 151.1. IR $(\mathrm{EtOH}) \vee$ 3085, 3031, 2990, 2935, 2894, 2815, 2225, 1627, 1597, $1521,1486,1464,1428,1403,1335,1178,1113,1057,1005,977,956,922,895,842,828,764$, 721, $694 \mathrm{~cm}^{-1}$. MS (ESI) m/z $276(\mathrm{M}+\mathrm{H})^{+}$. HRMS (ESI) calcd. for $\mathrm{C}_{19} \mathrm{H}_{18} \mathrm{NO}$ : 276.1383, Found: 276.1385 .
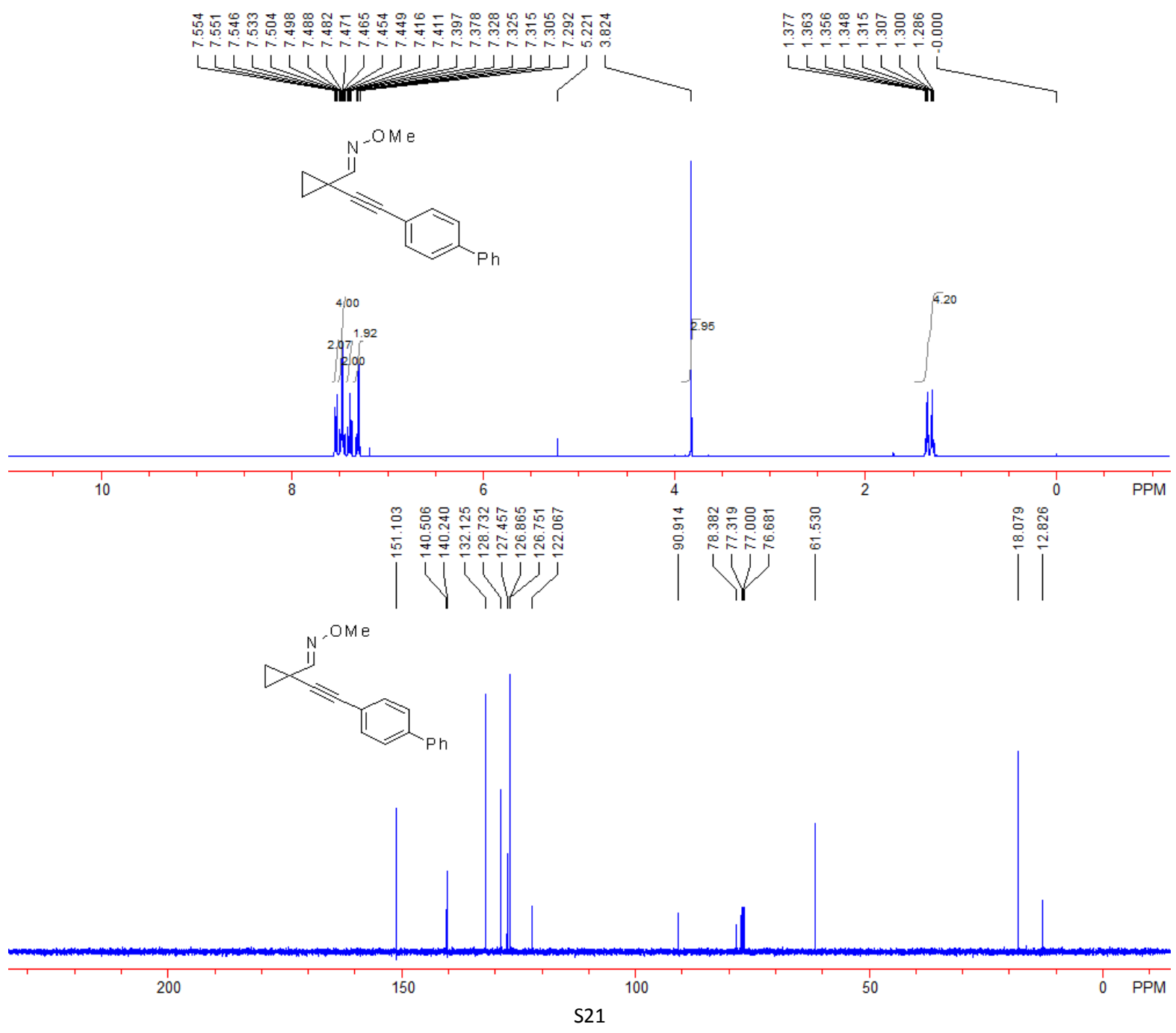


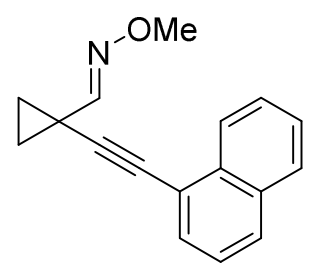

(E)-1-(naphthalen-1-ylethynyl)cyclopropane-1-carbaldehyde O-methyl oxime 1d: Yield: $225 \mathrm{mg}$, 90\%; A colorless oil; ${ }^{1} \mathrm{H}$ NMR $\left(\mathrm{CDCl}_{3}, 400 \mathrm{MHz}, \mathrm{TMS}\right) \delta 1.37-1.40(\mathrm{~m}, 2 \mathrm{H}, 2 \mathrm{CH}), 1.48-1.56(\mathrm{~m}$, 2H, 2CH), $3.89\left(\mathrm{~s}, 3 \mathrm{H}, \mathrm{CH}_{3}\right), 7.34(\mathrm{~s}, 1 \mathrm{H}, \mathrm{CH}), 7.37-7.41(\mathrm{~m}, 1 \mathrm{H}, \mathrm{Ar}), 7.50-7.56(\mathrm{~m}, 1 \mathrm{H}, \mathrm{Ar})$, 7.61-7.63 (m, 1H, Ar), 8.41 (d, $J=8.0 \mathrm{~Hz}, 1 \mathrm{H}, \mathrm{Ar}) .{ }^{13} \mathrm{C} \mathrm{NMR}\left(\mathrm{CDCl}_{3}, 100 \mathrm{MHz}, \mathrm{TMS}\right) \delta 13.2,18.1$, 61.6, 77.1, 95.1, 120.8, 125.1, 126.2, 126.3, 126.5, 128.1, 128.2, 130.0, 133.0, 133.5, 151.3. IR $(\mathrm{EtOH}) \vee 3010,2923,2854,2233,1501,1453,1363,1182$ 1105, 1051, 1027, 954, 839, 815, 774 $\mathrm{cm}^{-1}$. MS (ESI) $\mathrm{m} / z 250(\mathrm{M}+\mathrm{H})^{+}$. HRMS (ESI) calcd. for $\mathrm{C}_{17} \mathrm{H}_{16} \mathrm{NO}$ : 250.1226, Found: 250.1228 .

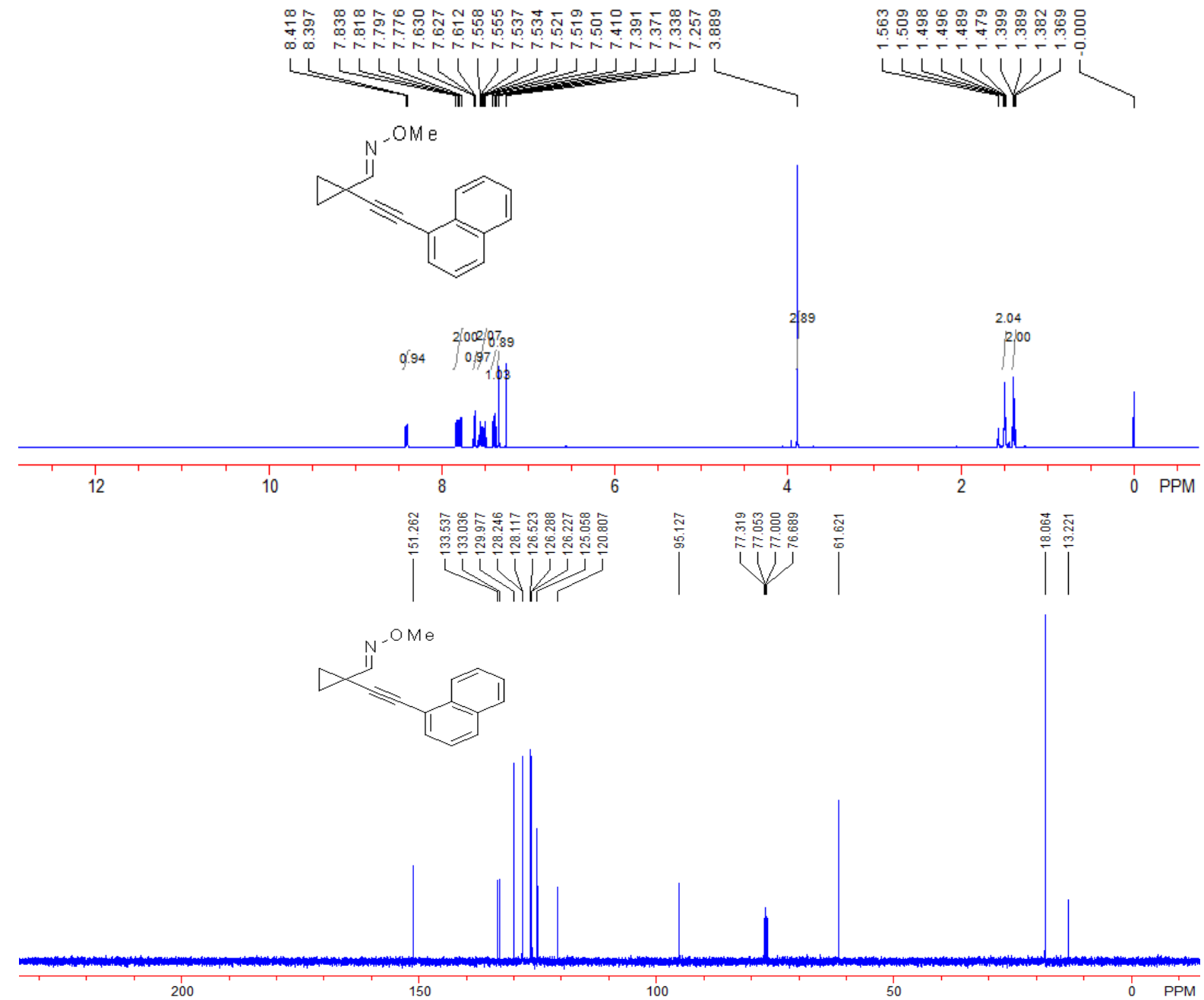




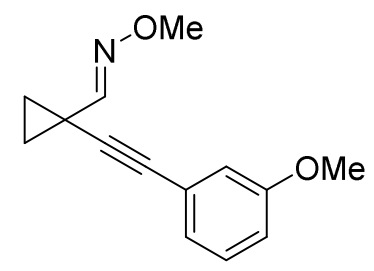

(E)-1-((3-methoxyphenyl)ethynyl)cyclopropane-1-carbaldehyde O-methyl oxime 1e: Yield: $211 \mathrm{mg}$, 92\%; A white solid, Mp: 50-52 ${ }^{\circ} \mathrm{C}$; ${ }^{1} \mathrm{H}$ NMR ( $\left.\mathrm{CDCl}_{3}, 400 \mathrm{MHz}, \mathrm{TMS}\right) \delta 1.17-1.28$ (m, 4H, 2CH ), $3.66\left(\mathrm{~s}, 3 \mathrm{H}, \mathrm{CH}_{3}\right), 3.79$ (s, 3H, $\left.\mathrm{CH}_{3}\right), 6.72-6.74(\mathrm{~m}, 1 \mathrm{H}, \mathrm{Ar}), 6.85$ (s, 1H, Ar), 6.91 (d, $J=6.8 \mathrm{~Hz}$, $1 \mathrm{H}, \mathrm{Ar}), 7.08(\mathrm{t}, J=8.0 \mathrm{~Hz}, 1 \mathrm{H}, \mathrm{Ar}), 7.19(\mathrm{~s}, 1 \mathrm{H}, \mathrm{CH}) .{ }^{13} \mathrm{C} \mathrm{NMR}\left(\mathrm{CDCl}_{3}, 100 \mathrm{MHz}, \mathrm{TMS}\right) \delta 12.7$, 17.9, 55.0, 61.4, 78.4, 90.0, 114.4, 116.4, 124.1, 124.2, 129.1, 151.0, 159.1. IR (EtOH) v 3002, 2964, 2938, 2899, 2816, 2228, 1569, 1492, 1442, 1424, 1263, 1243, 1225, 1102, 1056, 1031, 978, 937, 924, 841, 793, 754, $690 \mathrm{~cm}^{-1}$. MS (ESI) $\mathrm{m} / z 230(\mathrm{M}+\mathrm{H})^{+}$. HRMS (ESI) calcd. for $\mathrm{C}_{14} \mathrm{H}_{16} \mathrm{NO}_{2}$ : 230.1176, Found: 230.1180.

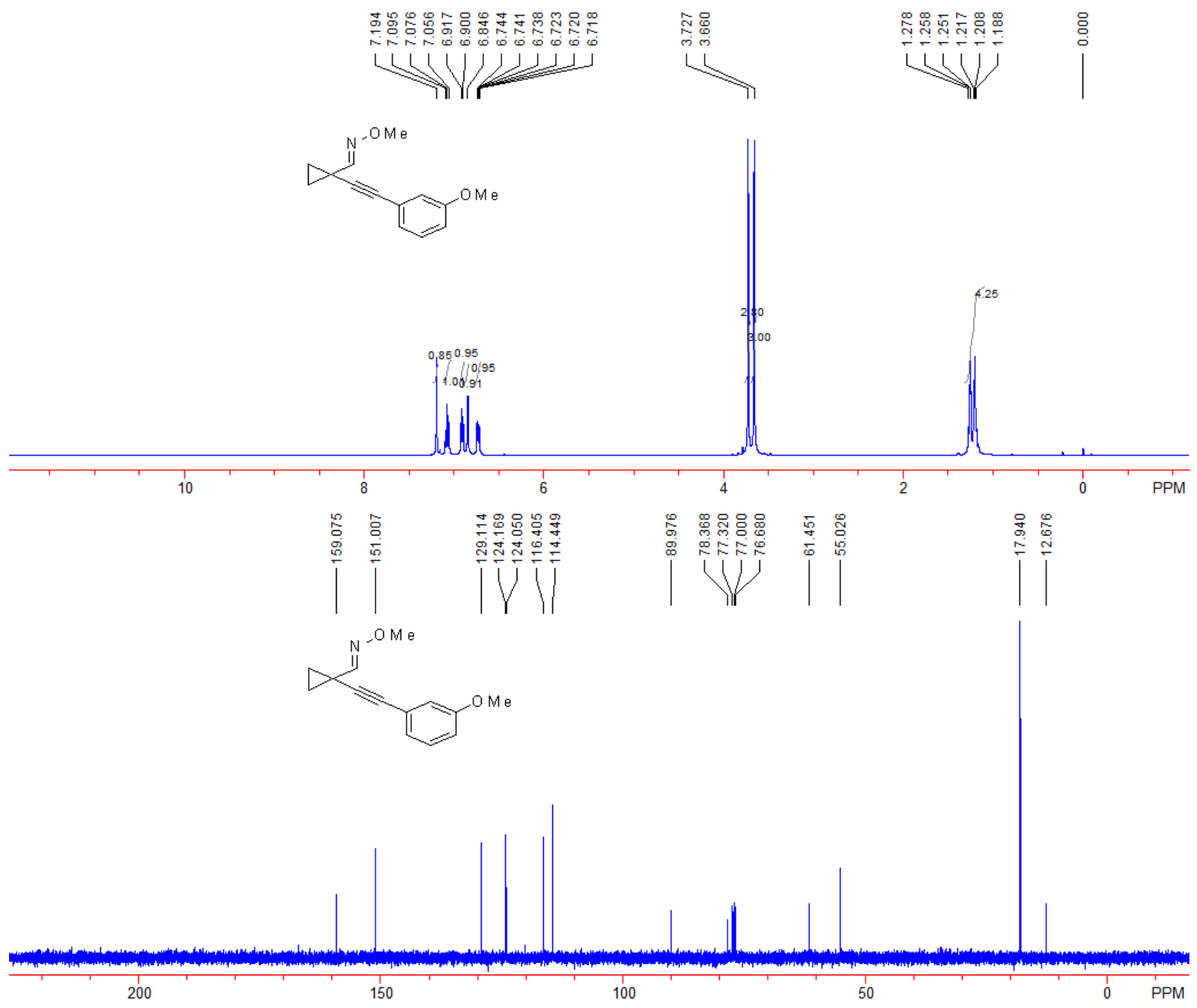




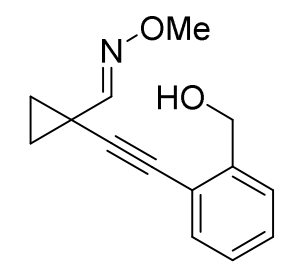

(E)-1-((2-(hydroxymethyl)phenyl)ethynyl)cyclopropane-1-carbaldehyde O-methyl oxime 1f: Yield: $208 \mathrm{mg}$, 91\%; A yellow oil; ${ }^{1} \mathrm{H}$ NMR ( $\left.\mathrm{CDCl}_{3}, 400 \mathrm{MHz}, \mathrm{TMS}\right) \delta 1.21-1.25$ (m, 2H, 2CH), 1.45-1.48 (m, 2H, 2CH), 3.88 (s, 3H, $\left.\mathrm{CH}_{3}\right), 4.25$ (br, 1H, OH), $4.74\left(\mathrm{~s}, 2 \mathrm{H}, \mathrm{CH}_{2}\right), 6.92(\mathrm{~s}, 1 \mathrm{H}, \mathrm{CH})$, 7.19-7.36 (m, 4H, Ar). ${ }^{13} \mathrm{C}$ NMR $\left(\mathrm{CDCl}_{3}, 100 \mathrm{MHz}, \mathrm{TMS}\right) \delta 13.5,17.0,61.6,64.2,79.1,122.0$, 127.4, 128.1, 128.3, 131.2, 143.9, 152.9. IR (EtOH) v 3431, 2936, 2850, 2816, 2230, 1735, 1485, 1451, 1380, 1238, 1054, 978, 903, 844, 757, $730 \mathrm{~cm}^{-1}$. MS (ESI) $\mathrm{m} / z 230(\mathrm{M}+\mathrm{H})^{+}$. HRMS (ESI) calcd. for $\mathrm{C}_{14} \mathrm{H}_{16} \mathrm{NO}_{2}: 230.1176$ Found: 230.1177 .

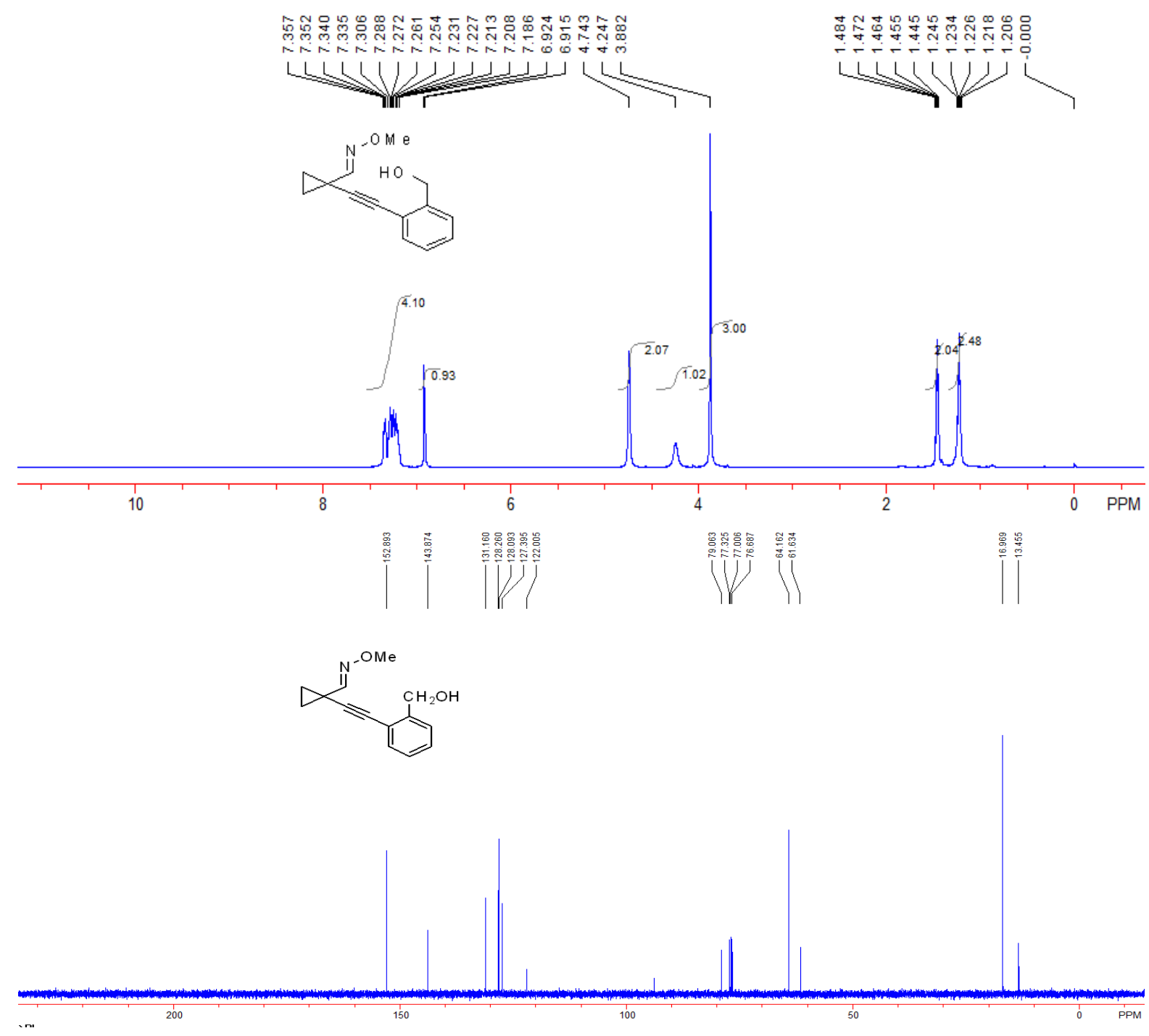




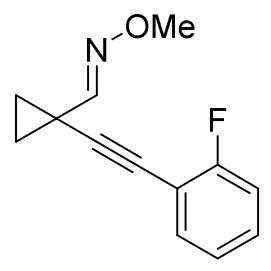

(E)-1-((2-fluorophenyl)ethynyl)cyclopropane-1-carbaldehyde O-methyl oxime 1g: Yield: $210 \mathrm{mg}$, 95\%; A yellow oil; ${ }^{1} \mathrm{H}$ NMR $\left(\mathrm{CDCl}_{3}, 400 \mathrm{MHz}, \mathrm{TMS}\right) \delta 1.31-1.40\left(\mathrm{~m}, 4 \mathrm{H}, 2 \mathrm{CH}_{2}\right), 3.74(\mathrm{~s}, 3 \mathrm{H}$, $\left.\mathrm{CH}_{3}\right)$, 7.00-7.06 (m, 2H, Ar), 7.21-7.24 (m, 1H, Ar), $7.30(\mathrm{~s}, 1 \mathrm{H}, \mathrm{CH}), 7.38-7.41(\mathrm{~m}, 1 \mathrm{H}, \mathrm{Ar}) .{ }^{13} \mathrm{C}$ $\mathrm{NMR}\left(\mathrm{CDCl}_{3}, 100 \mathrm{MHz}, \mathrm{TMS}\right) \delta$ 12.9, 18.2, 61.5, 71.8, 95.5 (d, $\left.J=30.0 \mathrm{~Hz}\right), 111.6$ (d, $J=30.0$ $\mathrm{Hz}), 115.2(\mathrm{~d}, J=20.8 \mathrm{~Hz}), 123.7(\mathrm{~d}, J=3.7 \mathrm{~Hz}), 129.5$, (d, $J=8.2 \mathrm{~Hz}), 133.6,150.7,162.8(\mathrm{~d}, J=$ 249.1 Hz). $\left.{ }^{19} \mathrm{~F} \mathrm{NMR} \mathrm{(376} \mathrm{MHz,} \mathrm{CDCl}_{3}, \mathrm{CFCl}_{3}\right) \delta-110.37-(-110.42)(\mathrm{m})$. IR (EtOH) v 3005, 2958, 2938, 2894, 2834, 2811, 2228, 1598, 1574, 1490, 1482, 1464, 1425, 1316, 1283, 1260, 1243, 1154 , $1058,1011,987,925,896,867,782,758,688 \mathrm{~cm}^{-1}$. MS (ESI) $m / z 218(\mathrm{M}+\mathrm{H})^{+}$. HRMS (ESI) calcd. for $\mathrm{C}_{13} \mathrm{H}_{13} \mathrm{FNO}$ : 218.0976, Found: 218.0978.

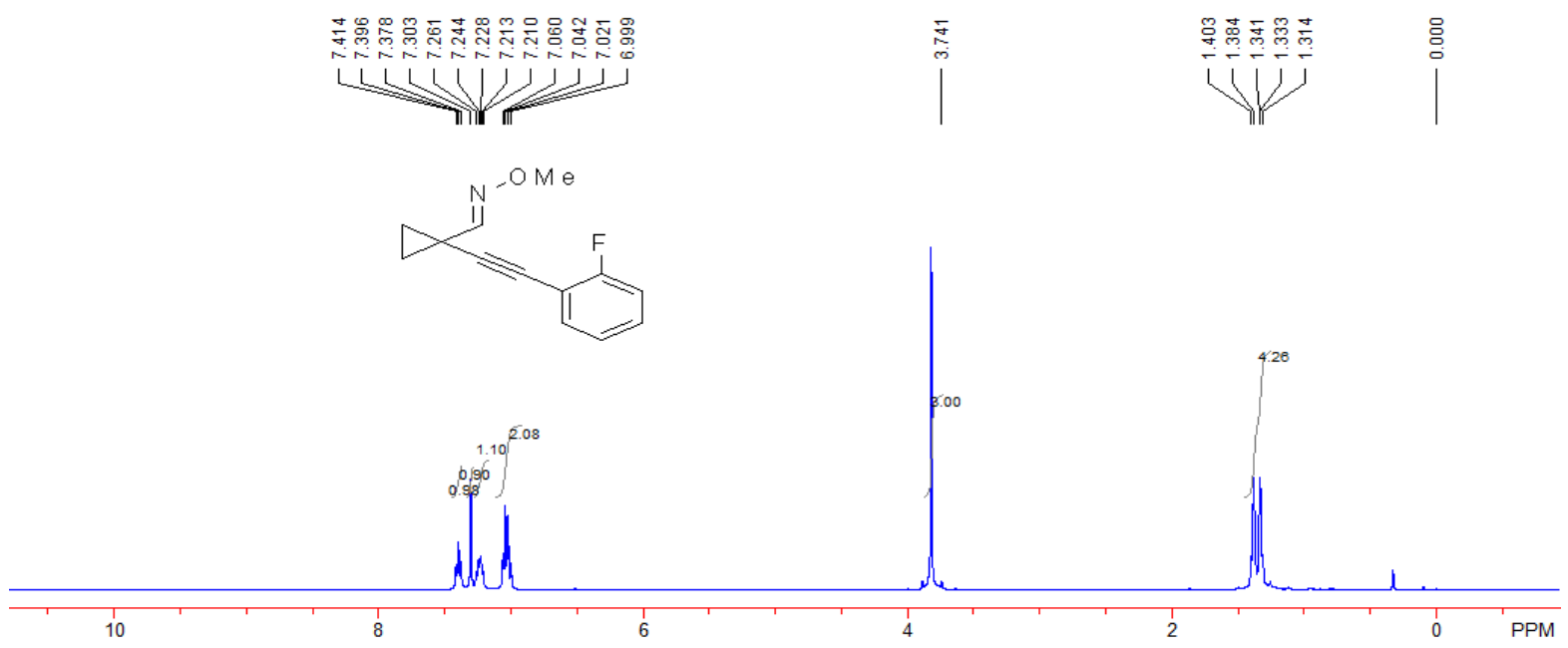



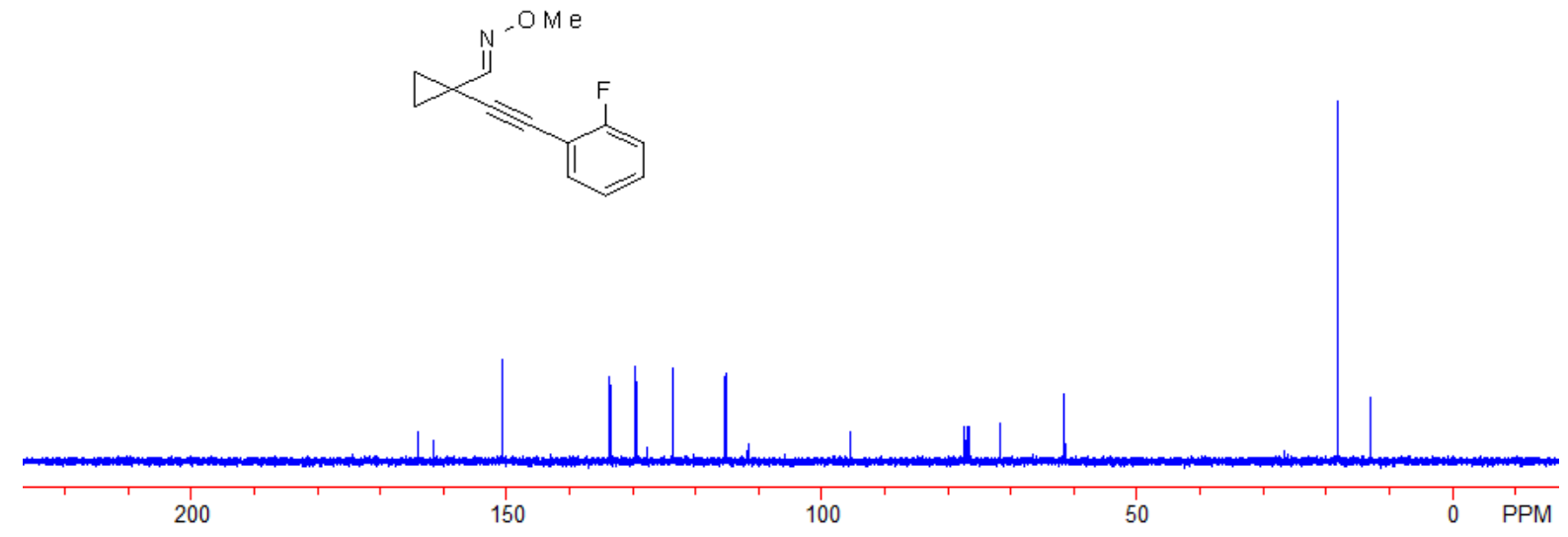

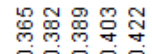

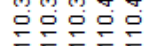

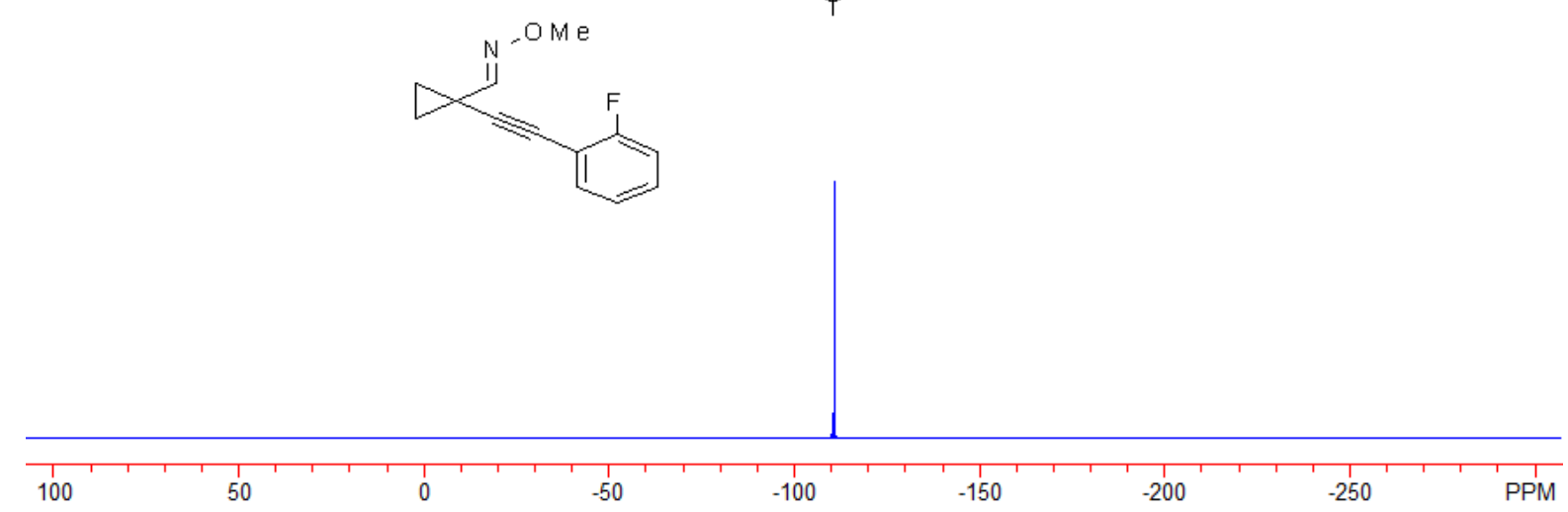

(ij

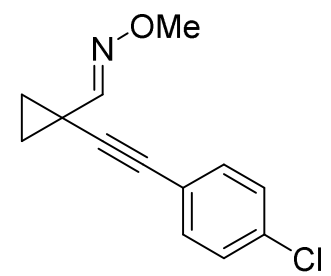

(E)-1-((4-chlorophenyl)ethynyl)cyclopropane-1-carbaldehyde O-methyl oxime 1h: Yield: $215 \mathrm{mg}$, 93\%; A white solid, Mp: 60-62 ${ }^{\circ} \mathrm{C} ;{ }^{1} \mathrm{H}$ NMR $\left(\mathrm{CDCl}_{3}, 400 \mathrm{MHz}, \mathrm{TMS}\right) \delta 1.29-1.37$ (m, 4H, 2CH ), $3.82\left(\mathrm{~s}, 3 \mathrm{H}, \mathrm{CH}_{3}\right), 7.22-7.28(\mathrm{~m}, 3 \mathrm{H}, 2 \mathrm{Ar}, 1 \mathrm{CH}), 7.30-7.33(\mathrm{~m}, 2 \mathrm{H}, \mathrm{Ar}) .{ }^{13} \mathrm{C} \mathrm{NMR}\left(\mathrm{CDCl}_{3}, 100\right.$ MHz, TMS) $\delta 12.7,18.0,61.5,77.5,91.2,121.7,128.4,132.9,133.8,150.9$. IR (EtOH) $\vee 3225$, 3016, 2935, 2226, 1662, 1592, 1579, 1488, 1456, 1420, 1396, 1359, 1321, 1307, 1277, 1257, 1234, 1192, 1165, 1091, 1036, 997, 956, 904, 844, 792, 735, 683, $671 \mathrm{~cm}^{-1}$. MS (ESI) $\mathrm{m} / z 234(\mathrm{M}+\mathrm{H})^{+}$. HRMS (ESI) calcd. for $\mathrm{C}_{13} \mathrm{H}_{13} \mathrm{CINO}$ : 234.0680, Found: 234.0681. 


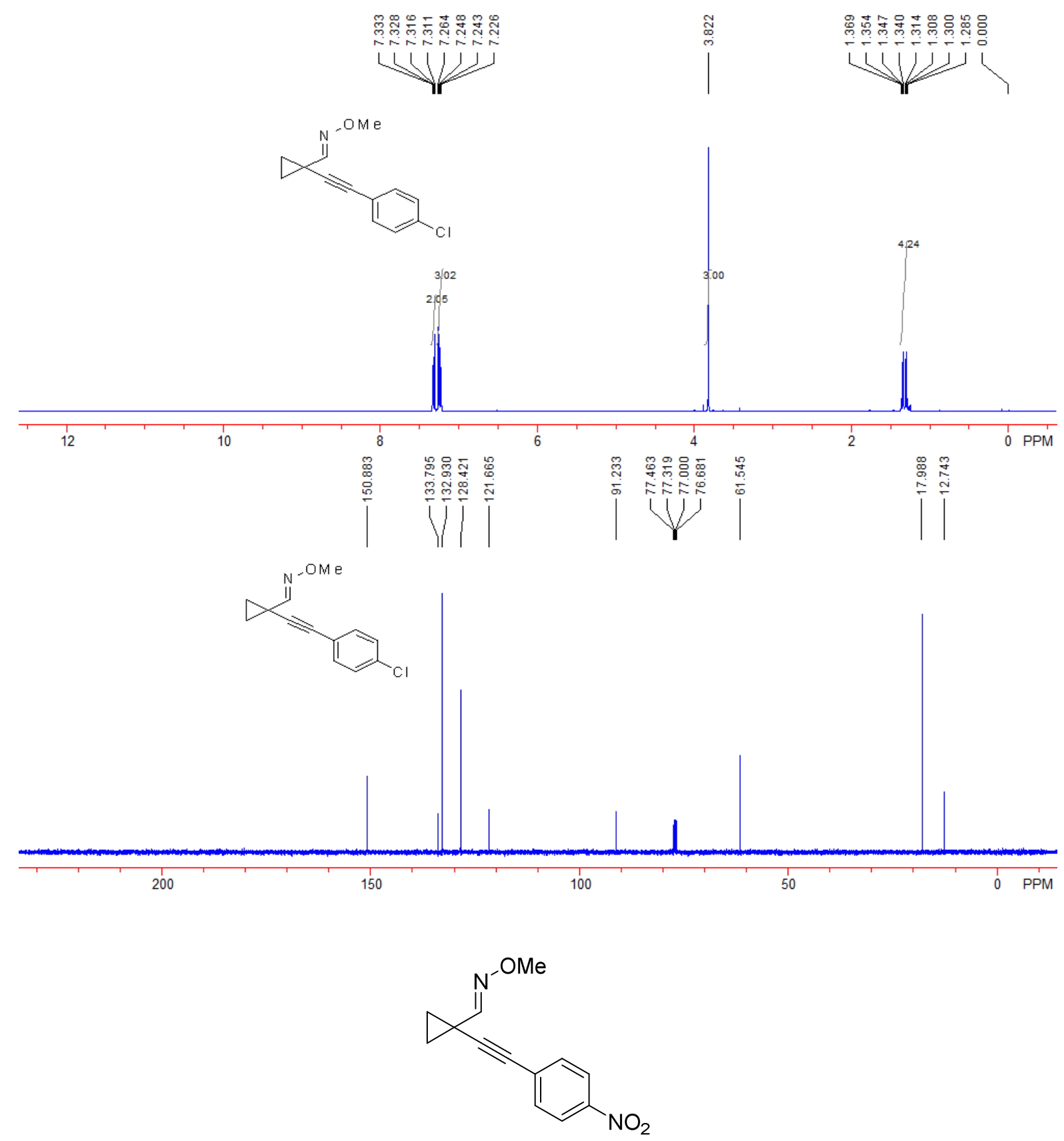

(E)-1-((4-nitrophenyl)ethynyl)cyclopropane-1-carbaldehyde O-methyl oxime 1i: Yield: $221 \mathrm{mg}$, 91\%; A yellow oil; ${ }^{1} \mathrm{H}$ NMR $\left(\mathrm{CDCl}_{3}, 400 \mathrm{MHz}, \mathrm{TMS}\right) \delta 1.28-1.37\left(\mathrm{~m}, 4 \mathrm{H}, 2 \mathrm{CH}_{2}\right), 3.77$ (s, 3H, $\left.\mathrm{CH}_{3}\right), 7.18$ (s, 1H, CH), 7.45-7.47 (m, 2H, Ar), 8.07-8.09 (m, 2H, Ar). ${ }^{13} \mathrm{C} \mathrm{NMR}\left(\mathrm{CDCl}_{3}, 100 \mathrm{MHz}\right.$, TMS) $\delta 12.9,18.3,61.7,77.3,96.2,123.4,130.3,132.4,146.7,150.4$. IR (EtOH) v 2966, 2936, 2896, 2855, 2224, 1593, 1516, 1492, 1464, 1324, 1284, 1175, 1107, 1058, 1012, 976, 899, 854, 749, 706, $688 \mathrm{~cm}^{-1}$. MS (ESI) $\mathrm{m} / z 245(\mathrm{M}+\mathrm{H})^{+}$. HRMS (ESI) calcd. for $\mathrm{C}_{13} \mathrm{H}_{13} \mathrm{~N}_{2} \mathrm{O}_{3}: 245.0921$, Found: 245.0921 . 

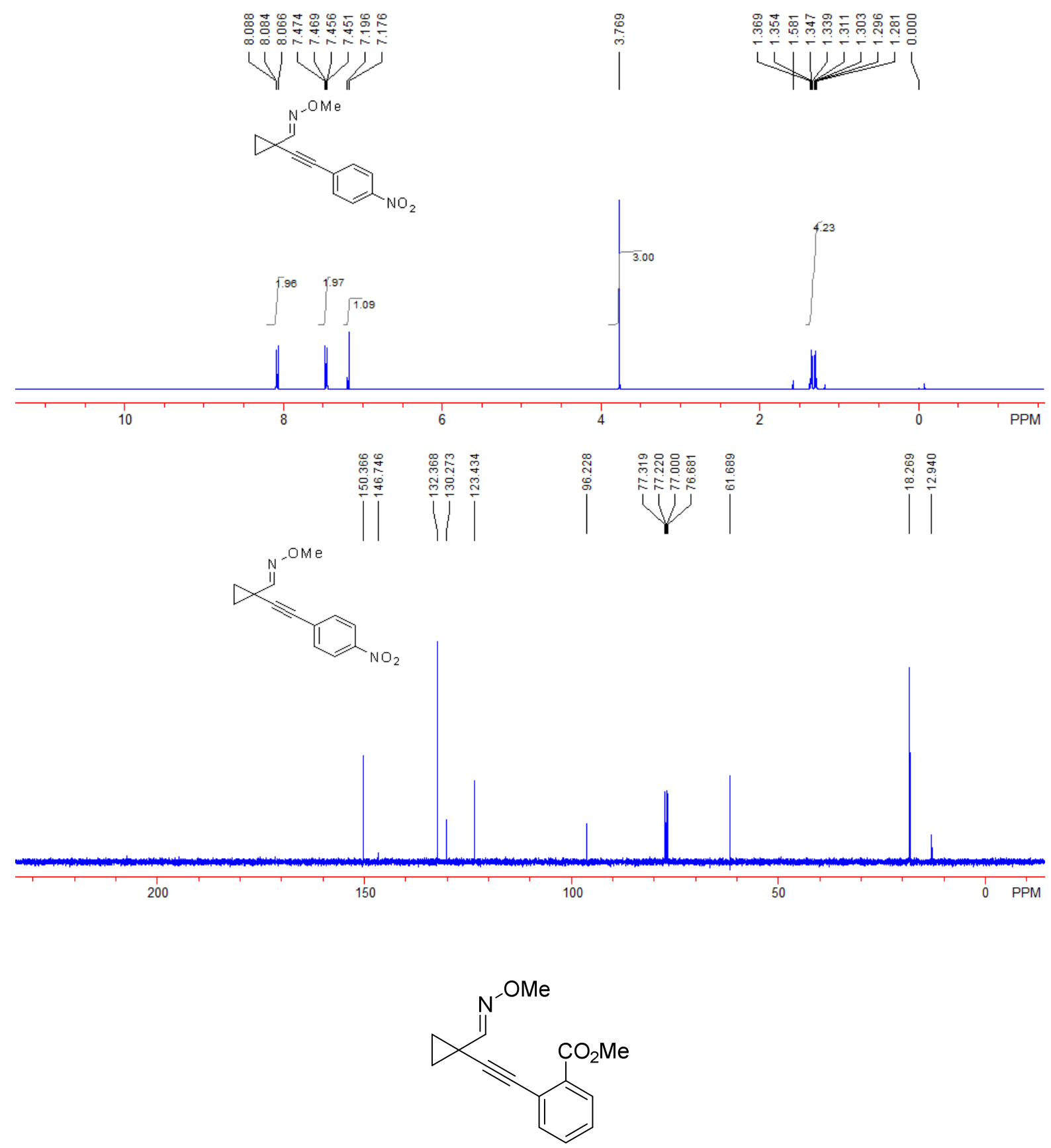

methyl (E)-2-((1-((methoxyimino)methyl)cyclopropyl)ethynyl)benzoate 1j: Yield: $232 \mathrm{mg}$, 90\%; A colorless oil; ${ }^{1} \mathrm{H}$ NMR $\left(\mathrm{CDCl}_{3}, 400 \mathrm{MHz}, \mathrm{TMS}\right) \delta 1.28-1.35\left(\mathrm{~m}, 4 \mathrm{H}, 2 \mathrm{CH}_{2}\right), 3.76\left(\mathrm{~s}, 3 \mathrm{H}, \mathrm{CH}_{3}\right), 3.83$ (s, 3H, $\left.\mathrm{CH}_{3}\right), 7.19-7.28(\mathrm{~m}, 2 \mathrm{H}, 1 \mathrm{Ar}, 1 \mathrm{CH}), 7.35$ (t, $\left.J=7.2 \mathrm{~Hz}, 21, \mathrm{Ar}\right), 7.44$ (d, J=7.6 Hz, 11, Ar), $7.82(\mathrm{~d}, J=7.6 \mathrm{~Hz}, 11, \mathrm{Ar}) .{ }^{13} \mathrm{C} \mathrm{NMR}\left(\mathrm{CDCl}_{3}, 100 \mathrm{MHz}, \mathrm{TMS}\right) \delta 13.2,18.6,52.0,61.6,77.2,95.9$, 123.7, 127.4, 130.3 131.5, 131.9, 134.1, 151.1, 166.7. IR (EtOH) v 2995, 2949, 2899, 2817, 2223, $1727,1596,1566,1485,1447,1432,1293,1270,1250,1189,1163,1129,1080,1057,1010,975$, 927, 898, 836, 813, 792, 755, 699, $654 \mathrm{~cm}^{-1}$. MS (ESI) $\mathrm{m} / z 258(\mathrm{M}+\mathrm{H})^{+}$. HRMS (ESI) calcd. for 
$\mathrm{C}_{15} \mathrm{H}_{16} \mathrm{NO}_{3}$ : 258.1125, Found: 258.1127.

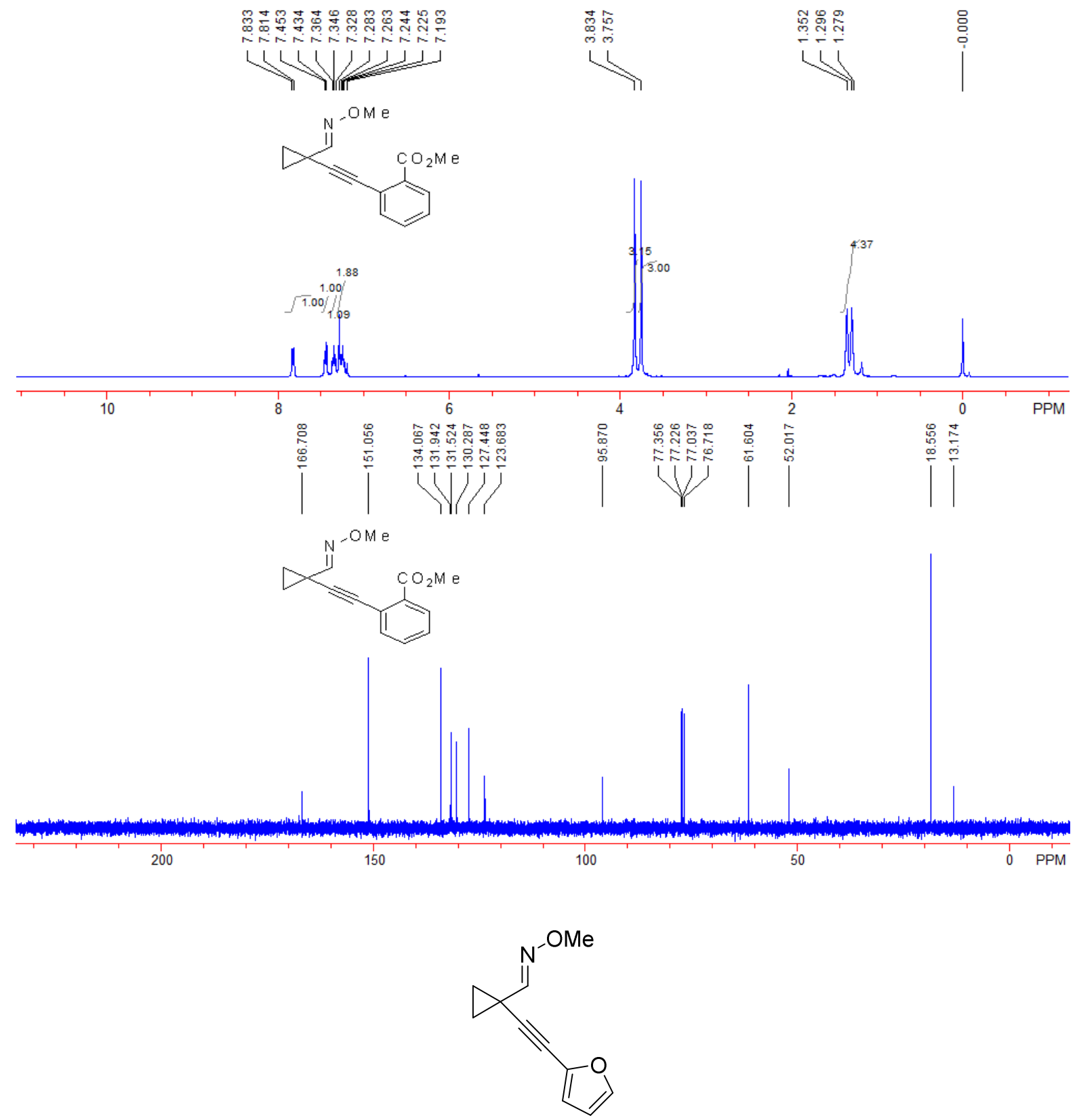

(E)-1-(furan-2-ylethynyl)cyclopropane-1-carbaldehyde O-methyl oxime 1k: Yield: 166 mg, 88\%; A yellow oil; ${ }^{1} \mathrm{H}$ NMR $\left(\mathrm{CDCl}_{3}, 400 \mathrm{MHz}, \mathrm{TMS}\right) \delta 1.32-1.38\left(\mathrm{~m}, 4 \mathrm{H}, 2 \mathrm{CH}_{2}\right), 3.82\left(\mathrm{~s}, 3 \mathrm{H}, \mathrm{CH}_{3}\right)$,

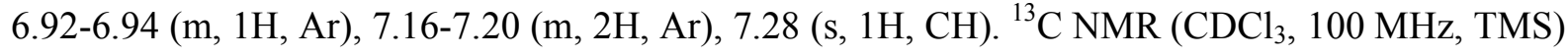
$\delta 12.7,18.3,61.6,68.7,94.6,110.7,115.0,136.9,143.1,150.5 . \mathrm{IR}(\mathrm{EtOH}) \vee 2992,2938,2817$, 2226, 1732, 1627, 1574, 1488, 1465, 1425, 1376, 1295, 1219, 1183, 1150, 1057, 1015, 977, 954, 931, 885, 812, 795, $741 \mathrm{~cm}^{-1}$. MS (ESI) $\mathrm{m} / z 190(\mathrm{M}+\mathrm{H})^{+}$. HRMS (ESI) calcd. for $\mathrm{C}_{11} \mathrm{H}_{12} \mathrm{NO}_{2}$ : 190.0863, Found: 190.0870. 


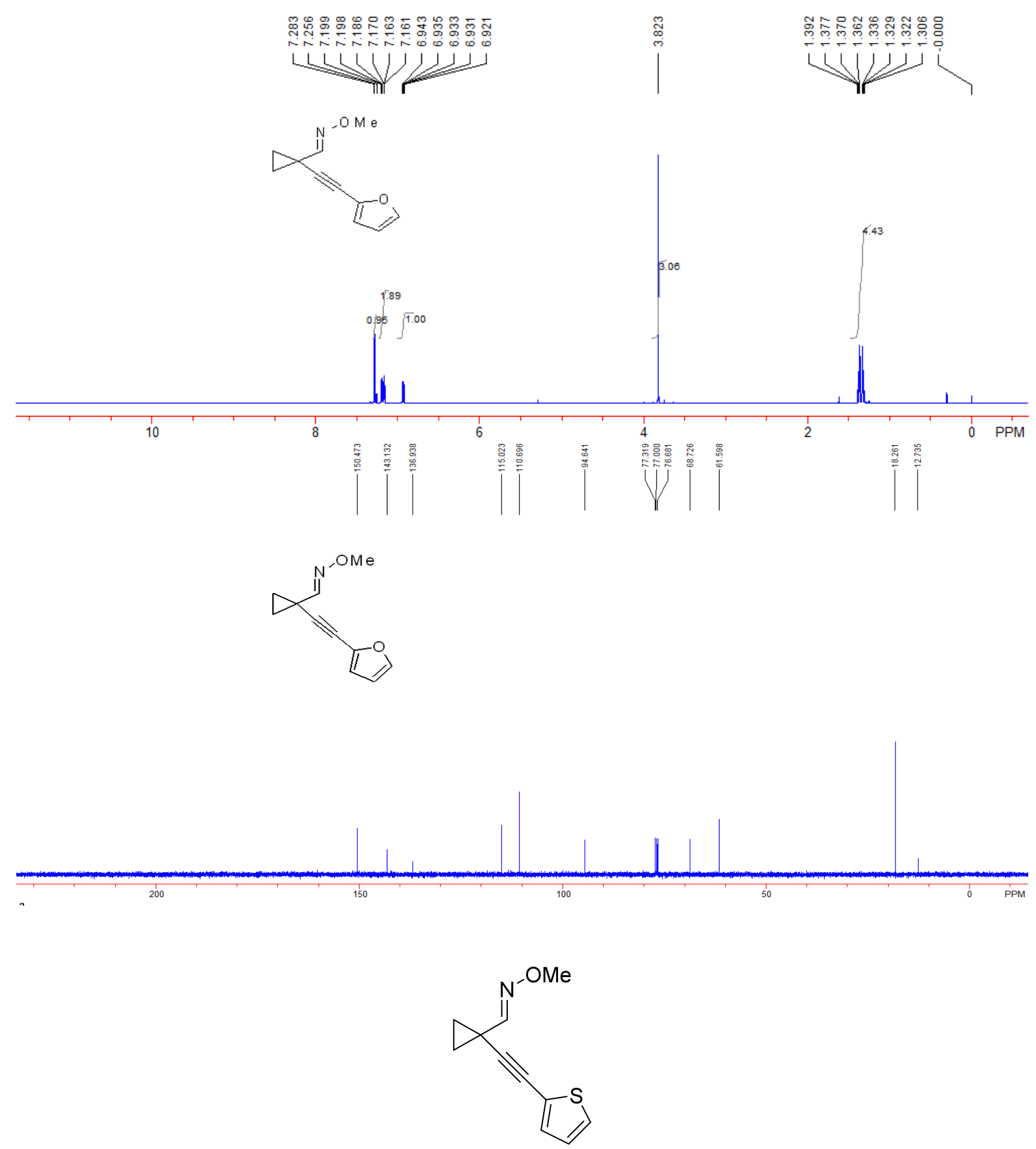

(E)-1-(thiophen-2-ylethynyl)cyclopropane-1-carbaldehyde O-methyl oxime 1l: Yield: $189 \mathrm{mg}, 93 \%$; A red oil; ${ }^{1} \mathrm{H}$ NMR $\left(\mathrm{CDCl}_{3}, 400 \mathrm{MHz}, \mathrm{TMS}\right) \delta 1.31-1.40\left(\mathrm{~m}, 4 \mathrm{H}, 2 \mathrm{CH}_{2}\right), 3.81\left(\mathrm{~s}, 3 \mathrm{H}, \mathrm{CH}_{3}\right)$, 6.33-6.35 (m, 1H, Ar), 6.52-6.53 (m, 1H, Ar), 7.25 (s, 1H, CH), 7.32-7.33 (m, 1H, Ar). ${ }^{13} \mathrm{C}$ NMR $\left(\mathrm{CDCl}_{3}, 100 \mathrm{MHz}, \mathrm{TMS}\right) \delta 13.0,18.2,61.6,71.7,94.2,123.3,126.6,132.0,150.9 . \mathrm{IR}(\mathrm{EtOH}) v$ 3106, 2997, 2961, 2937, 2899, 2816, 2222, 1626, 1517, 1464, 1428, 1334, 1221, 1160, 1057, 1009, 967, 918, 888, 850, 831, 760, $699 \mathrm{~cm}^{-1}$. MS (ESI) $\mathrm{m} / z 206(\mathrm{M}+\mathrm{H})^{+}$. HRMS (ESI) calcd. for $\mathrm{C}_{11} \mathrm{H}_{12} \mathrm{NOS}: 206.0634$, Found: 206.0642. 


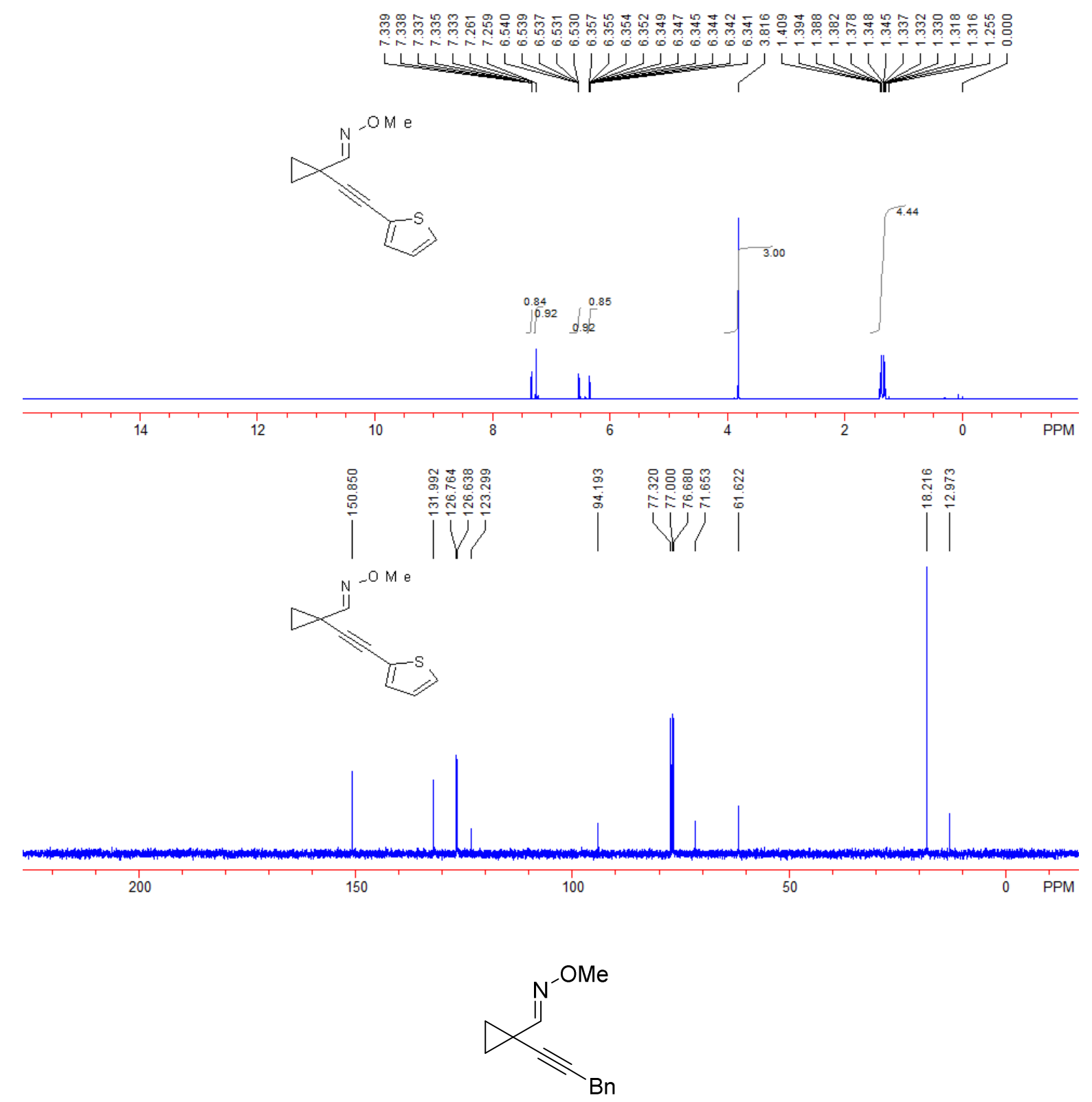

(E)-1-(3-phenylprop-1-yn-1-yl)cyclopropane-1-carbaldehyde O-methyl oxime 1m: Yield: 158 mg, 74\%; A yellow oil; ${ }^{1} \mathrm{H}$ NMR $\left(\mathrm{CDCl}_{3}, 400 \mathrm{MHz}, \mathrm{TMS}\right) \delta 1.15-1.27\left(\mathrm{~m}, 4 \mathrm{H}, 2 \mathrm{CH}_{2}\right), 3.61$ (s, 2H, $\left.\mathrm{CH}_{2}\right), 3.83\left(\mathrm{~s}, 3 \mathrm{H}, \mathrm{CH}_{3}\right), 7.17(\mathrm{~s}, 1 \mathrm{H}, \mathrm{CH}), 7.22-7.25$ (m, 1H, Ar), 7.28-7.36 (m, 4H, Ar). ${ }^{13} \mathrm{C} \mathrm{NMR}$ $\left(\mathrm{CDCl}_{3}, 100 \mathrm{MHz}, \mathrm{TMS}\right) \delta 12.5,17.4,25.1,61.5,76.4,82.7,126.4,127.8,128.4,136.9,152.0 . \mathrm{IR}$ $(\mathrm{EtOH}) \vee 3070,3049,2959,2923,2852,1726,1631,1494,1455,1428,1260,1053,1022,845,800$, 729, $699 \mathrm{~cm}^{-1}$. MS (ESI) $m / z 214(\mathrm{M}+\mathrm{H})^{+}$. HRMS (ESI) calcd. for $\mathrm{C}_{14} \mathrm{H}_{16} \mathrm{NO}$ : 214.1226, Found: 214.1226. 

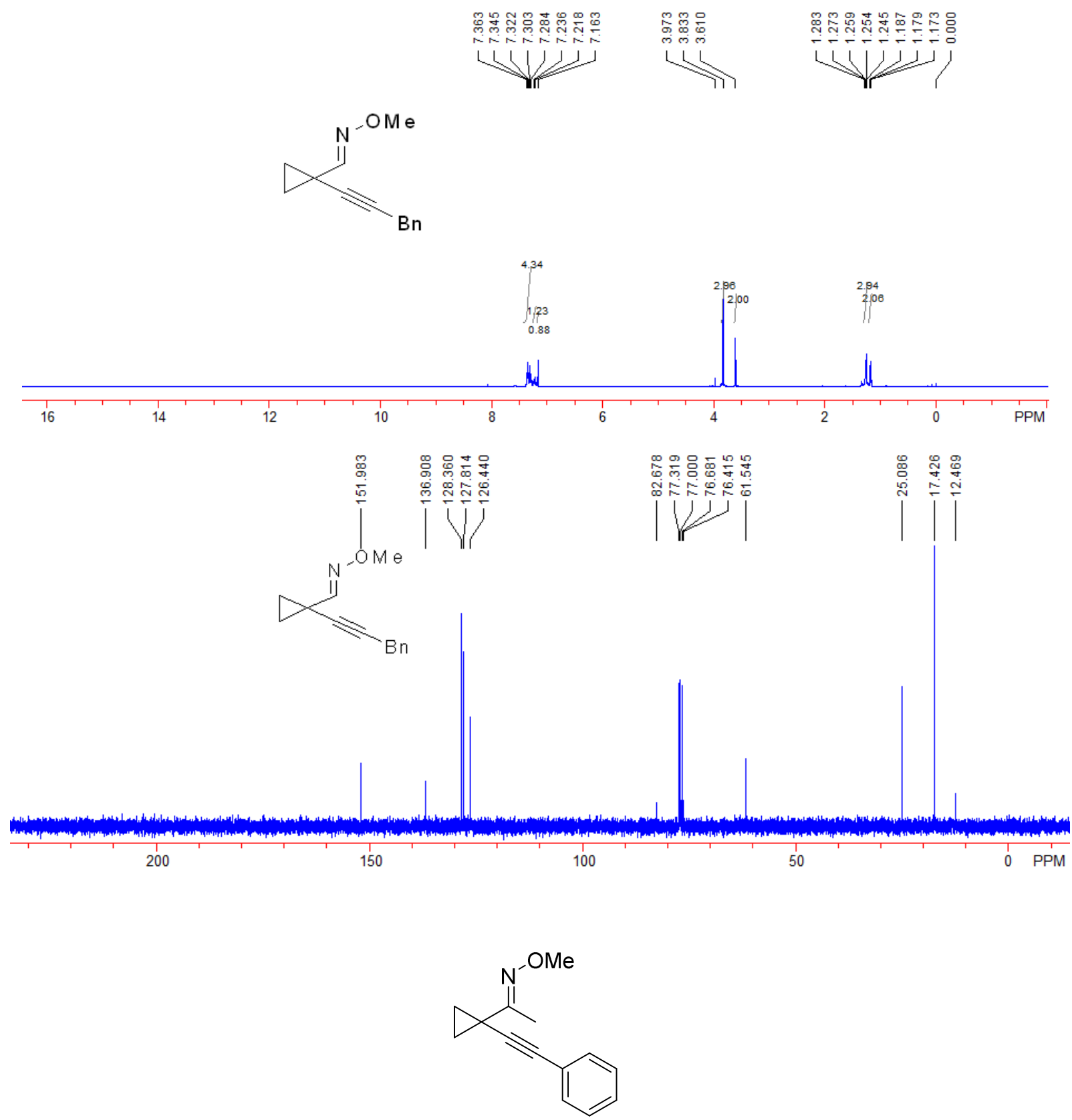

(E)-1-(1-(phenylethynyl)cyclopropyl)ethan-1-one O-methyl oxime 1n: Yield: $190 \mathrm{mg}$, 88\%; A colorless oil; ${ }^{1} \mathrm{H}$ NMR $\left(\mathrm{CDCl}_{3}, 400 \mathrm{MHz}, \mathrm{TMS}\right) \delta$ 1.21-1.22 (m, 2H, 2CH), 1.36-1.37 (m, 2H, 2CH), 2.07 (s, 3H, $\left.\mathrm{CH}_{3}\right), 3.82\left(\mathrm{~s}, 3 \mathrm{H}, \mathrm{CH}_{3}\right), 7.25-7.27$ (m, 3H, Ar), 7.37-7.39 (m, 2H, Ar). ${ }^{13} \mathrm{C}$ NMR $\left(\mathrm{CDCl}_{3}, 100 \mathrm{MHz}, \mathrm{TMS}\right) \delta$ 13.6, 16.8, 17.1, 61.4, 91.6, 123.4, 127.8, 128.1, 131.6, 155.9. IR $(\mathrm{EtOH}) \vee 3080,3054,2958,2936,2898,2816,2227,1598,1491,1464,1442,1367,1306,1144$, 1052, 952, 903, 879, 852, 755, $690 \mathrm{~cm}^{-1}$. MS (ESI) $m / z 214(\mathrm{M}+\mathrm{H})^{+}$. HRMS (ESI) calcd. for $\mathrm{C}_{14} \mathrm{H}_{16} \mathrm{NO}: 214.1226$, Found: 214.1229. 

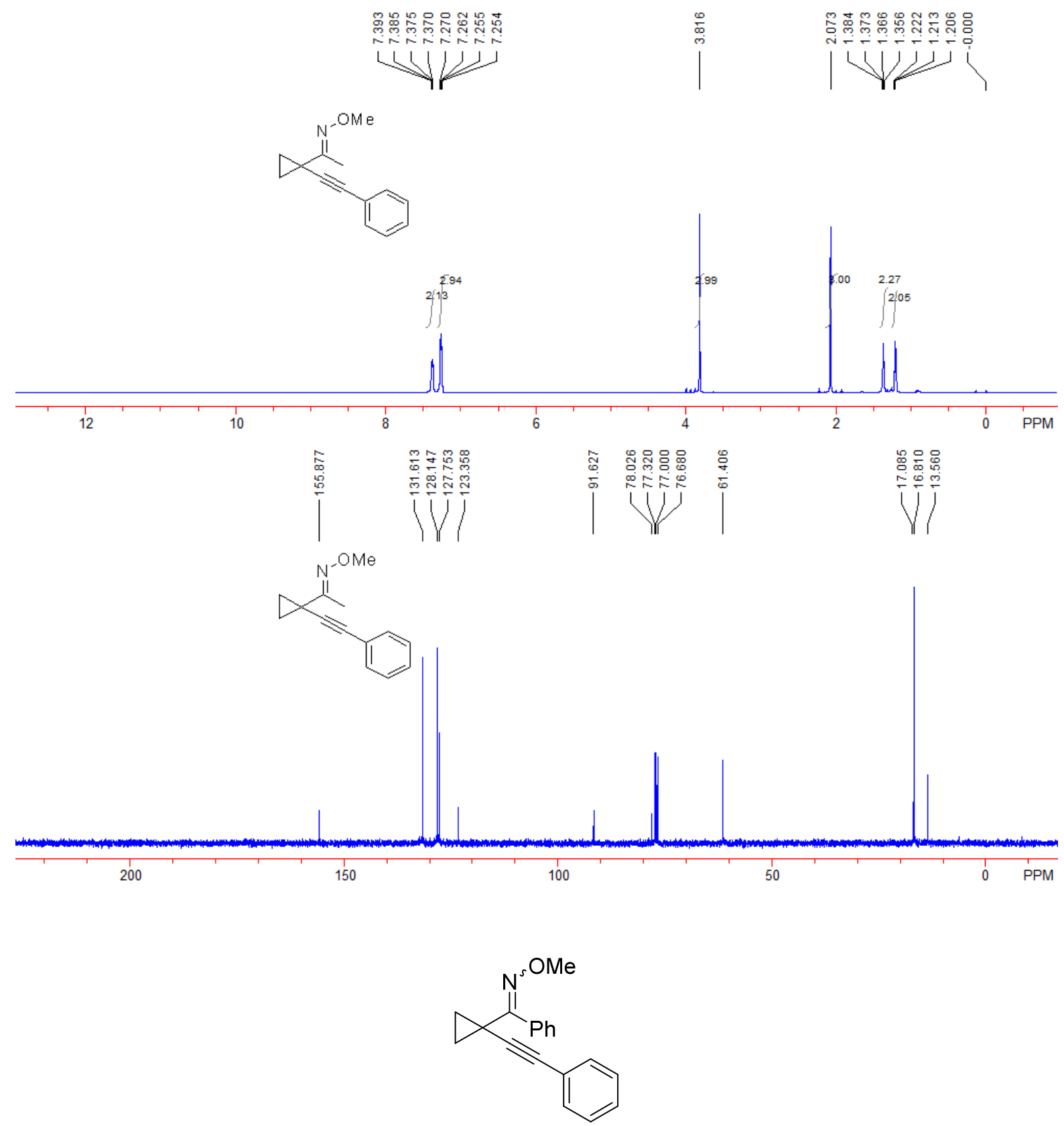

phenyl(1-(phenylethynyl)cyclopropyl)methanone O-methyl oxime 1o: The oxime ether was obtained as a light yellow oil (74\% yield), a 1.7/1 mixture of $\mathrm{E} / \mathrm{Z}$ isomers which could not been separated by column chromatography. ${ }^{1} \mathrm{H}$ NMR $\left(\mathrm{CDCl}_{3}, 400 \mathrm{MHz}, \mathrm{TMS}\right)$ major isomer: $\delta$ 1.32-1.35 (m, 2H, 2CH), 1.52-1.54 (m, 2H, 2CH), 3.82 (s, 3H, CH3), 7.16 (br, 2H, Ar), 7.00-7.22 (m, 2H, Ar), 7.32-7.41 (m, 4H, Ar), 7.55-7.57 (m, 2H, Ar). ${ }^{13} \mathrm{C}$ NMR ( $\left.\mathrm{CDCl}_{3}, 100 \mathrm{MHz}, \mathrm{TMS}\right) \delta$ $10.8,17.0,17.2,17.9,62.0,62.5,76.0,78.7,91.97,91.99,123.3,123.4,126.9,127.5,127.6,127.8$, $127.96,128.00,128.1,128.4,128.8,128.9,131.3,131.6,133.0,134.7,154.2,155.9$. IR (EtOH) $\delta$ 
3070, 2959, 2923, 2852, 1726, 1631, 1494, 1455, 1428, 1260, 1053, 1022, 845, 800, 729, $699 \mathrm{~cm}^{-1}$. MS (ESI) m/z $276(\mathrm{M}+\mathrm{H})+$. HRMS (ESI) calcd. for $\mathrm{C}_{19} \mathrm{H}_{18} \mathrm{NO}: 276.1383$, Found: 276.1386.
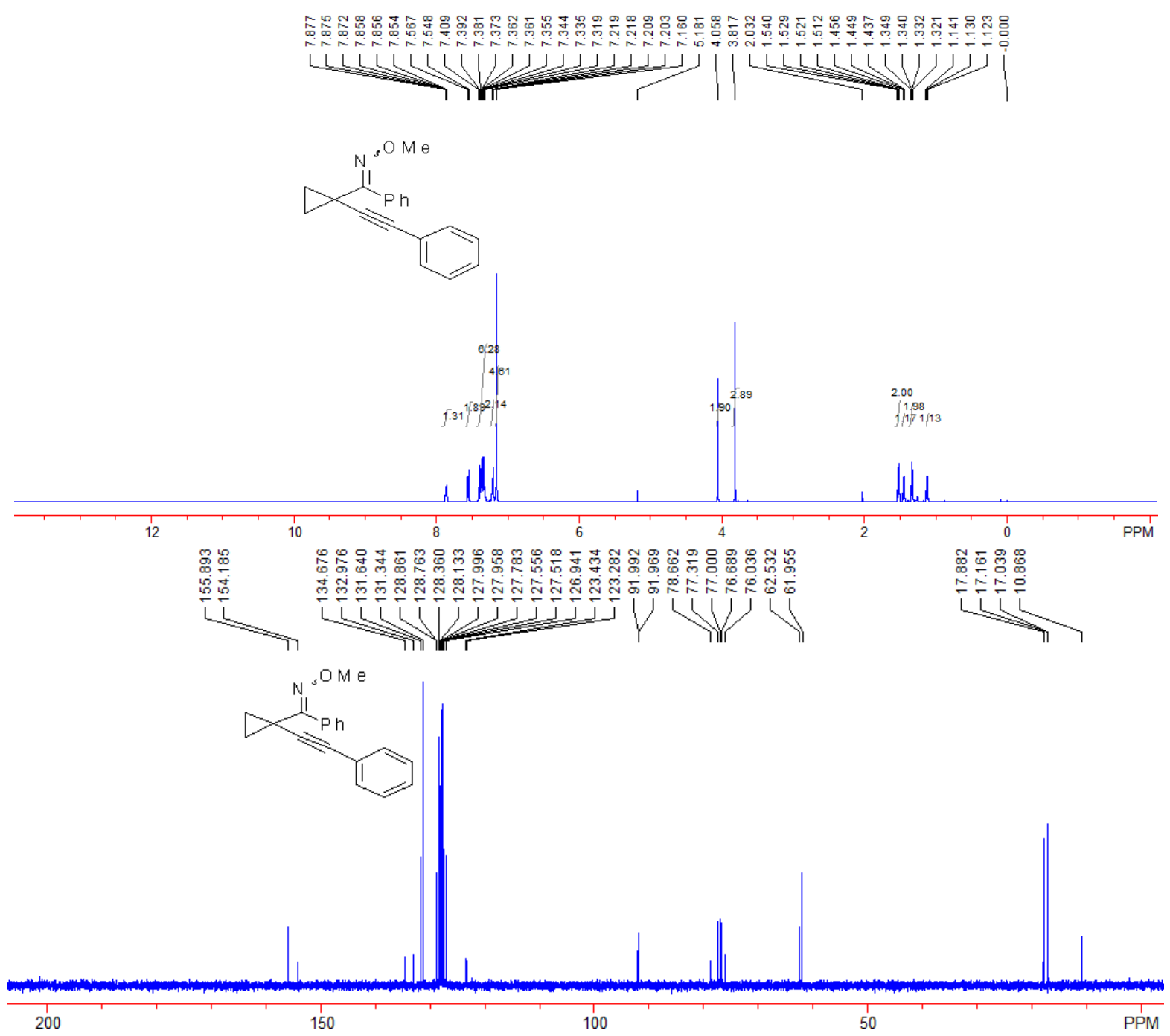

(E)-1-(-2-phenyl-1-(phenylethynyl)cyclopropyl)ethan-1-one O-methyl oxime $\quad \mathbf{1 p} \quad$ and (E)-1-(phenylethynyl)bicyclo[4.1.0]heptan-2-one O-methyl oxime 1q were known compounds. ${ }^{4}$

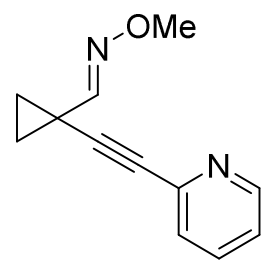

(E)-1-(pyridin-2-ylethynyl)cyclopropane-1-carbaldehyde O-methyl oxime 1r: Yield: $183 \mathrm{mg}, 91 \%$; A red oil; ${ }^{1} \mathrm{H}$ NMR $\left(\mathrm{CDCl}_{3}, 400 \mathrm{MHz}, \mathrm{TMS}\right) \delta 1.33-1.36(\mathrm{~m}, 2 \mathrm{H}, 2 \mathrm{CH}), 1.43-1.46(\mathrm{~m}, 2 \mathrm{H}, 2 \mathrm{CH})$, 
$3.82\left(\mathrm{~s}, 3 \mathrm{H}, \mathrm{CH}_{3}\right), 7.17-7.21(\mathrm{~m}, 1 \mathrm{H}, \mathrm{Ar}), 7.32(\mathrm{~s}, 1 \mathrm{H}, \mathrm{CH}), 7.39-7.41(\mathrm{~m}, 1 \mathrm{H}, \mathrm{Ar})$, 7.59-7.64 (m, $1 \mathrm{H}, \mathrm{Ar}), 8.52-8.53$ (m, 1H, Ar). ${ }^{13} \mathrm{C} \mathrm{NMR}\left(\mathrm{CDCl}_{3}, 100 \mathrm{MHz}, \mathrm{TMS}\right) \delta 12.4,18.0,61.3,77.8,90.5$, 122.3, 126.8, 135.8, 142.9, 149.5, 150.3. IR (EtOH) v 2961, 2929, 2847, 1707, 1527, 1474, 1412, 1276, 1208, 1181, 1136, 1084, 1047, 1014, 977, 924, 880, 757, 697, $676 \mathrm{~cm}^{-1}$. MS (ESI) $\mathrm{m} / z 201$ $(\mathrm{M}+\mathrm{H})^{+}$. HRMS (ESI) calcd. for $\mathrm{C}_{12} \mathrm{H}_{13} \mathrm{~N}_{2} \mathrm{O}: 201.1022$, Found: 201.1024.

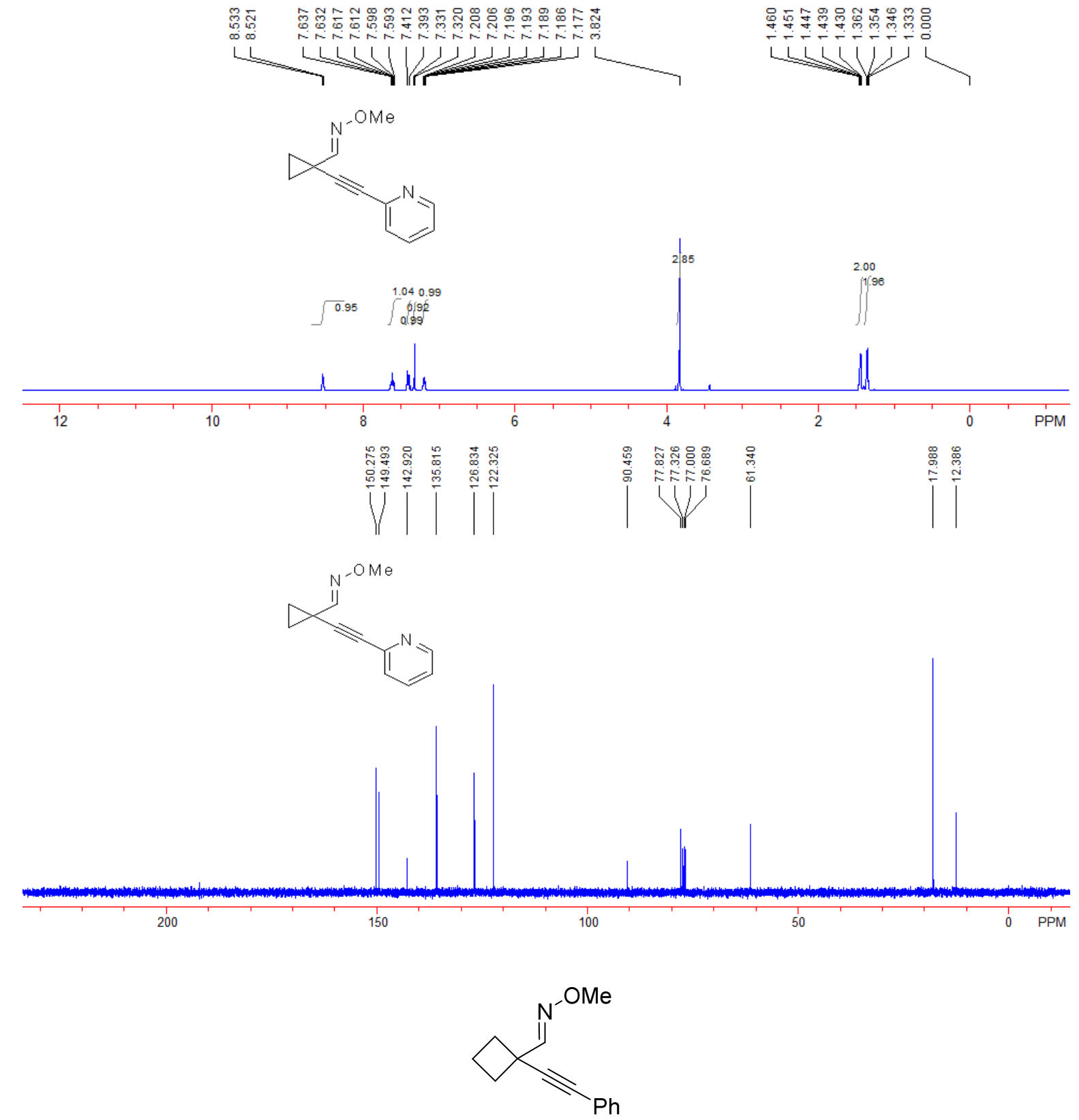

(E)-1-(phenylethynyl)cyclobutane-1-carbaldehyde O-methyl oxime 1s: Yield: $194 \mathrm{mg}$, 91\%; A colorless oil; ${ }^{1} \mathrm{H}$ NMR $\left(\mathrm{CDCl}_{3}, 400 \mathrm{MHz}, \mathrm{TMS}\right) \delta$ 1.94-2.18 (m, 2H, $\left.\mathrm{CH}_{2}\right), 2.41-2.59(\mathrm{~m}, 4 \mathrm{H}$, $\left.2 \mathrm{CH}_{2}\right), 3.88\left(\mathrm{~s}, 3 \mathrm{H}, \mathrm{CH}_{3}\right), 7.26-7.30(\mathrm{~m}, 3 \mathrm{H}, \mathrm{Ar}), 7.40-7.43(\mathrm{~m}, 2 \mathrm{H}, \mathrm{Ar}), 7.51(\mathrm{~s}, 1 \mathrm{H}, \mathrm{CH}) .{ }^{13} \mathrm{C}$ 
NMR $\left(\mathrm{CDCl}_{3}, 100 \mathrm{MHz}, \mathrm{TMS}\right) \delta 16.5,33.2,37.2,61.6,83.3,92.0,123.3,127.9,128.2,131.6$, 150.9. IR $(\mathrm{EtOH}) \vee 2937,2854,2816,1598,1490,1463,1442,1333,1287,1183,1053,914,883$, 812, 755, $690 \mathrm{~cm}^{-1}$. MS (ESI) $\mathrm{m} / z 214(\mathrm{M}+\mathrm{H})^{+}$. HRMS (ESI) calcd. for $\mathrm{C}_{14} \mathrm{H}_{16} \mathrm{NO}: 214.1226$, Found: 214.1224.

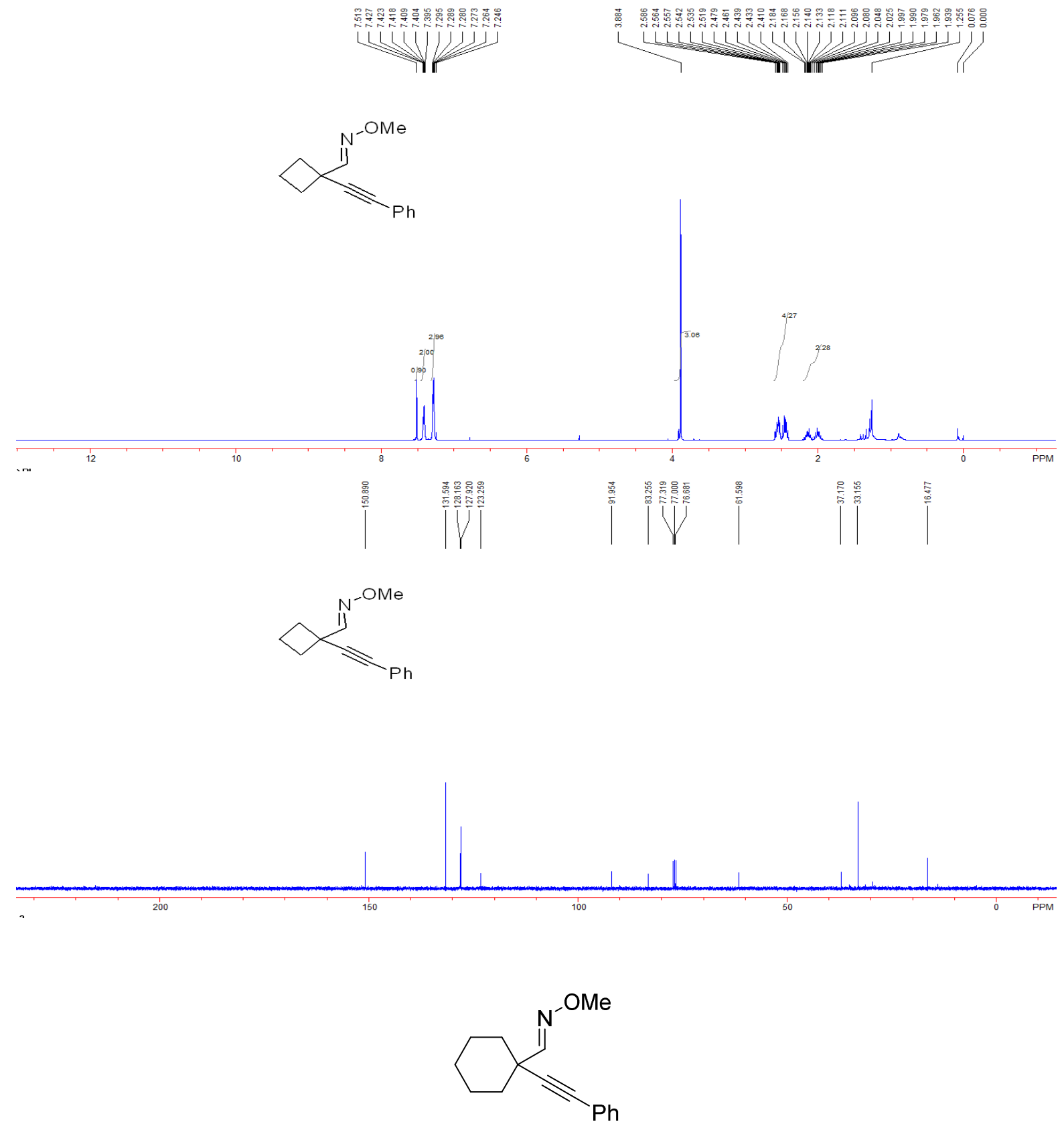

(E)-1-(phenylethynyl)cyclohexane-1-carbaldehyde O-methyl oxime 1t: Yield: $190 \mathrm{mg}, 84 \%$; A colorless oil; ${ }^{1} \mathrm{H}$ NMR $\left(\mathrm{CDCl}_{3}, 400 \mathrm{MHz}, \mathrm{TMS}\right) \delta$ 1.57-1.80 (m, 8H, 3CH $\left.2,2 \mathrm{CH}\right), 1.94-1.97$ (m, $2 \mathrm{H}, 2 \mathrm{CH}), 3.86\left(\mathrm{~s}, 3 \mathrm{H}, \mathrm{CH}_{3}\right), 7.25-7.29(\mathrm{~m}, 3 \mathrm{H}, \mathrm{Ar}), 7.41(\mathrm{~s}, 1 \mathrm{H}, \mathrm{CH}), 7.42-7.44(\mathrm{~m}, 2 \mathrm{H}, \mathrm{Ar}) .{ }^{13} \mathrm{C}$ $\mathrm{NMR}\left(\mathrm{CDCl}_{3}, 100 \mathrm{MHz}, \mathrm{TMS}\right) \delta 22.4,25.6,35.3,38.9,61.5,84.4,90.8,123.3,127.9,128.1,131.6$, 
153.7. IR (EtOH) $\vee 2931,2855,1741,1706,1572,1442,1415,1260,1229,1048,970,924,880$, 798, 755, $690 \mathrm{~cm}^{-1}$. MS (ESI) $\mathrm{m} / z$ 243(M+H) ${ }^{+}$. HRMS (ESI) calcd. for $\mathrm{C}_{16} \mathrm{H}_{19} \mathrm{O}_{2}: 243.1380$, Found: 243.1382 .
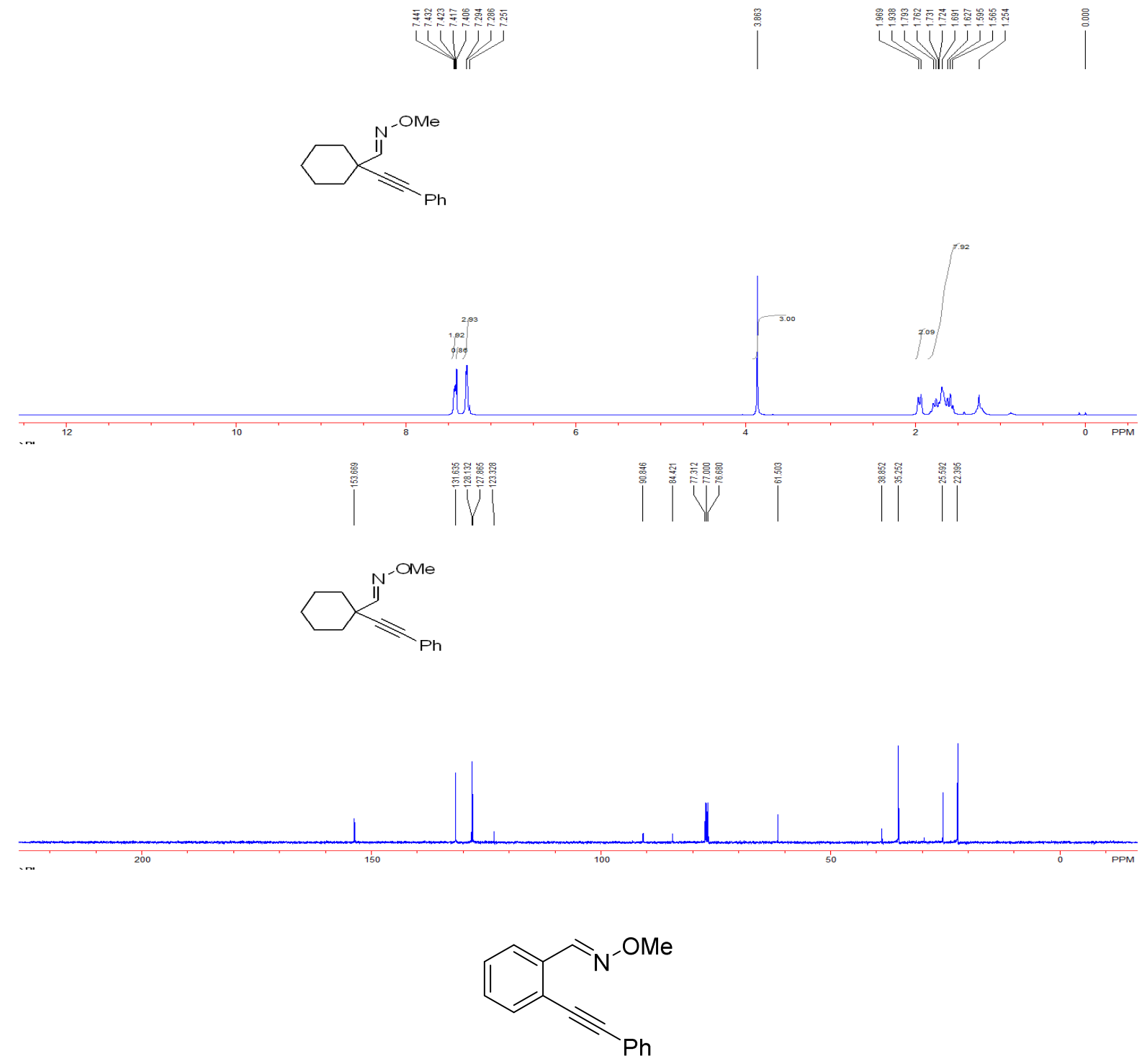

(E)-2-(phenylethynyl)benzaldehyde O-methyl oxime 1u: Yield: $179 \mathrm{mg}, 79 \%$; A yellow oil; ${ }^{1} \mathrm{H}$ NMR $\left(\mathrm{CDCl}_{3}, 400 \mathrm{MHz}, \mathrm{TMS}\right) \delta 4.00\left(\mathrm{~s}, 3 \mathrm{H}, \mathrm{CH}_{3}\right), 7.30-7.35(\mathrm{~m}, 5 \mathrm{H}, \mathrm{Ar}), 7.52-7.55$ (m, 3H, Ar), 7.91-7.93 (m, 1H, Ar), 8.66 (s, 1H, CH). ${ }^{13} \mathrm{C} \mathrm{NMR}\left(\mathrm{CDCl}_{3}, 100 \mathrm{MHz}, \mathrm{TMS}\right) \delta$ 62.1, 86.2, 94.8, $122.7,122.9,125.1,128.4,128.5,128.6,129.4,131.5,132.4,133.1,147.2$. IR (EtOH) $v 2916,2850$, 1739, 1559, 1492, 1472, 1440, 1422, 1260, 1054, 922, 756, $689 \mathrm{~cm}^{-1}$. MS (ESI) $\mathrm{m} / z 236(\mathrm{M}+\mathrm{H})^{+}$. HRMS (ESI) calcd. for $\mathrm{C}_{16} \mathrm{H}_{14} \mathrm{NO}$ : 236.1070, Found: 236.1071 . 


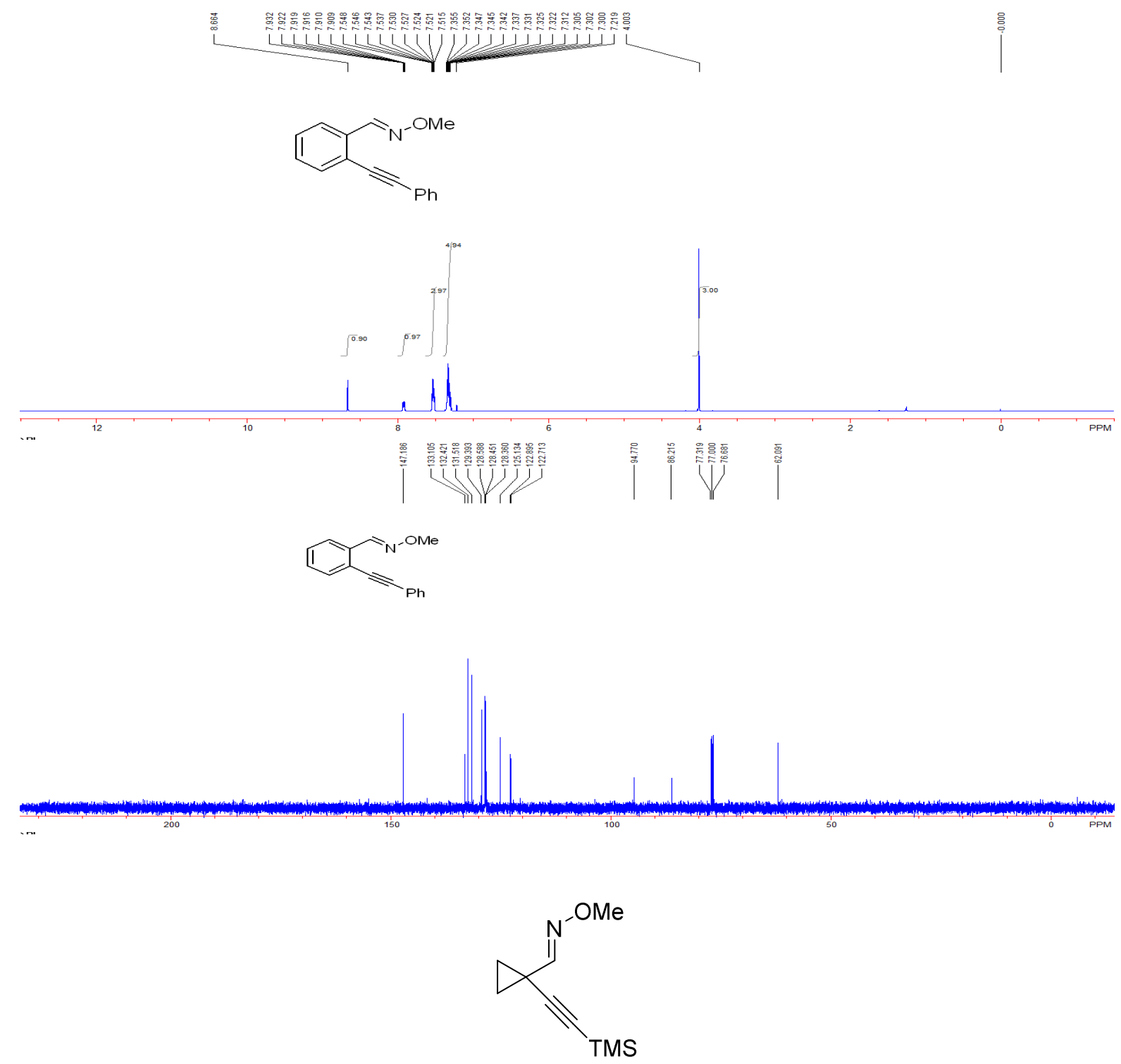

(E)-1-((trimethylsilyl)ethynyl)cyclopropane-1-carbaldehyde O-methyl oxime 1v: Yield: $171 \mathrm{mg}$, 88\%; A colorless oil; ${ }^{1} \mathrm{H}$ NMR $\left(\mathrm{CDCl}_{3}, 400 \mathrm{MHz}, \mathrm{TMS}\right) \delta 0.15\left(\mathrm{~s}, 9 \mathrm{H}, 3 \mathrm{CH}_{3}\right), 1.24-1.26(\mathrm{~m}, 4 \mathrm{H}$,

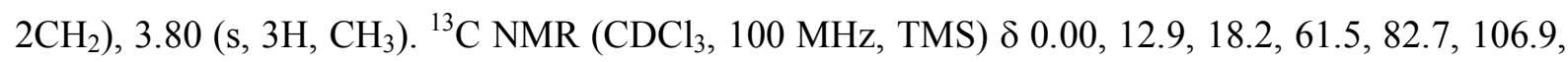
150.9. IR $(\mathrm{EtOH}) \vee 2959,2900,2163,1465,1250,1063,965,898,842,760,699 \mathrm{~cm}^{-1} . \mathrm{MS}(\mathrm{ESI})$ $m / z 196(\mathrm{M}+\mathrm{H})^{+}$. HRMS (ESI) calcd. for $\mathrm{C}_{10} \mathrm{H}_{18} \mathrm{NOSi}$ : 196.1152, Found: 196.1154. 


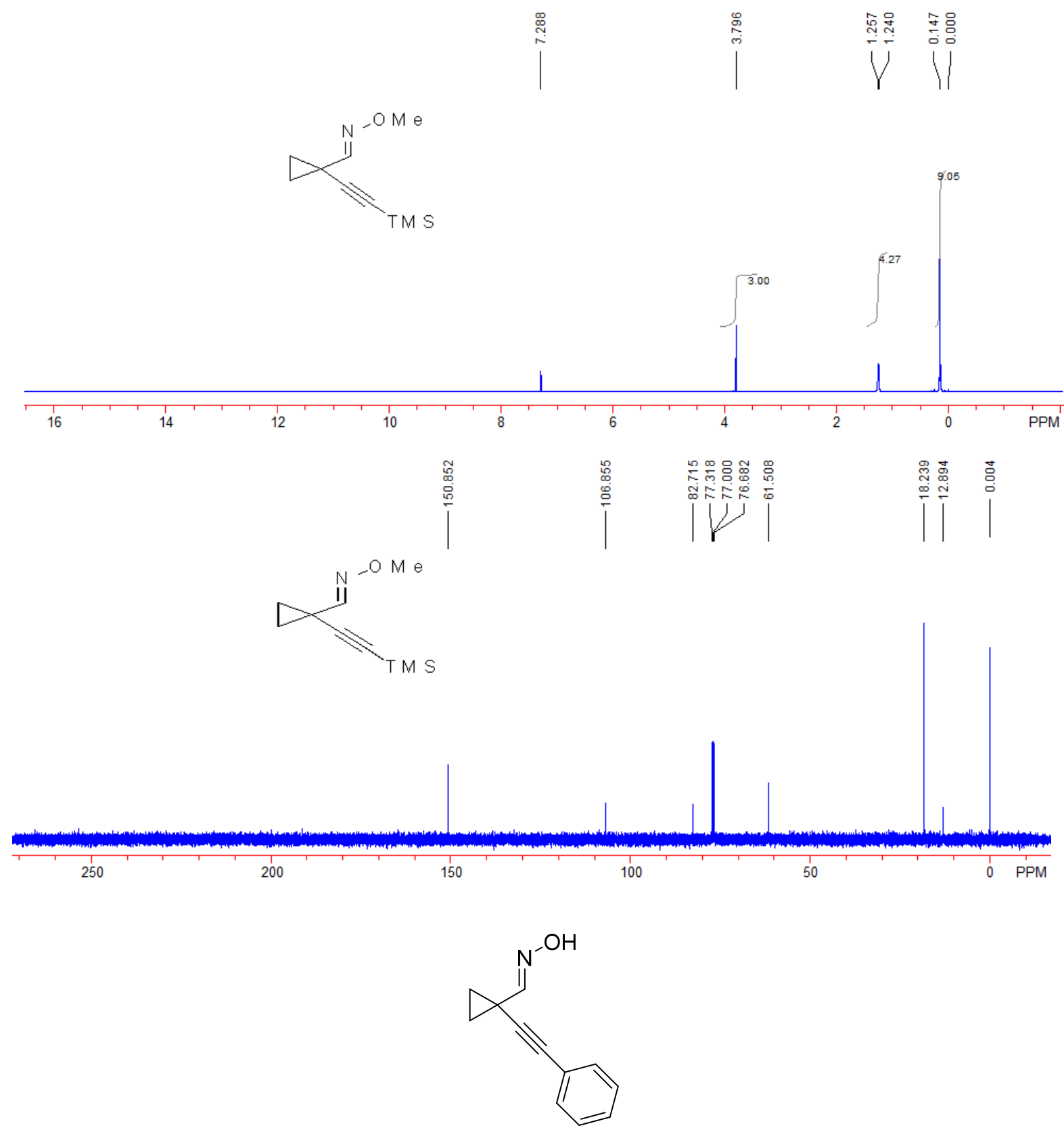

(E)-1-(phenylethynyl)cyclopropane-1-carbaldehyde oxime 4a: Yield: $154 \mathrm{mg}, 83 \%$; A white solid, Mp: $133-135{ }^{\circ} \mathrm{C} ;{ }^{1} \mathrm{H}$ NMR $\left(\mathrm{CDCl}_{3}, 400 \mathrm{MHz}, \mathrm{TMS}\right) \delta 1.21-1.28(\mathrm{~m}, 2 \mathrm{H}, 2 \mathrm{CH}), 1.41-1.44(\mathrm{~m}, 2 \mathrm{H}$, 2CH), $7.06(\mathrm{~s}, 1 \mathrm{H}, \mathrm{CH}), 7.26-7.28(\mathrm{~m}, 3 \mathrm{H}, \mathrm{Ar}), 7.41-7.43$ (m, 2H, Ar), 9.21 (br, 1H, OH). ${ }^{13} \mathrm{C}$ NMR $\left(\mathrm{CDCl}_{3}, 100 \mathrm{MHz}, \mathrm{TMS}\right) \delta 13.4,17.3,79.6,89.1,122.9,127.9,128.1,131.7,151.2$. IR (neat) $v$ $3214,3147,2918,1663,1510,1455,1427,1303,1236,1066,1037,992,944,914,851,815,732$ $\mathrm{cm}^{-1}$. MS (ESI) m/z $200(\mathrm{M}+\mathrm{H})^{+}$. HRMS (ESI) calcd. for $\mathrm{C}_{12} \mathrm{H}_{12} \mathrm{NO}$ : 186.0913, Found: 186.0913. 


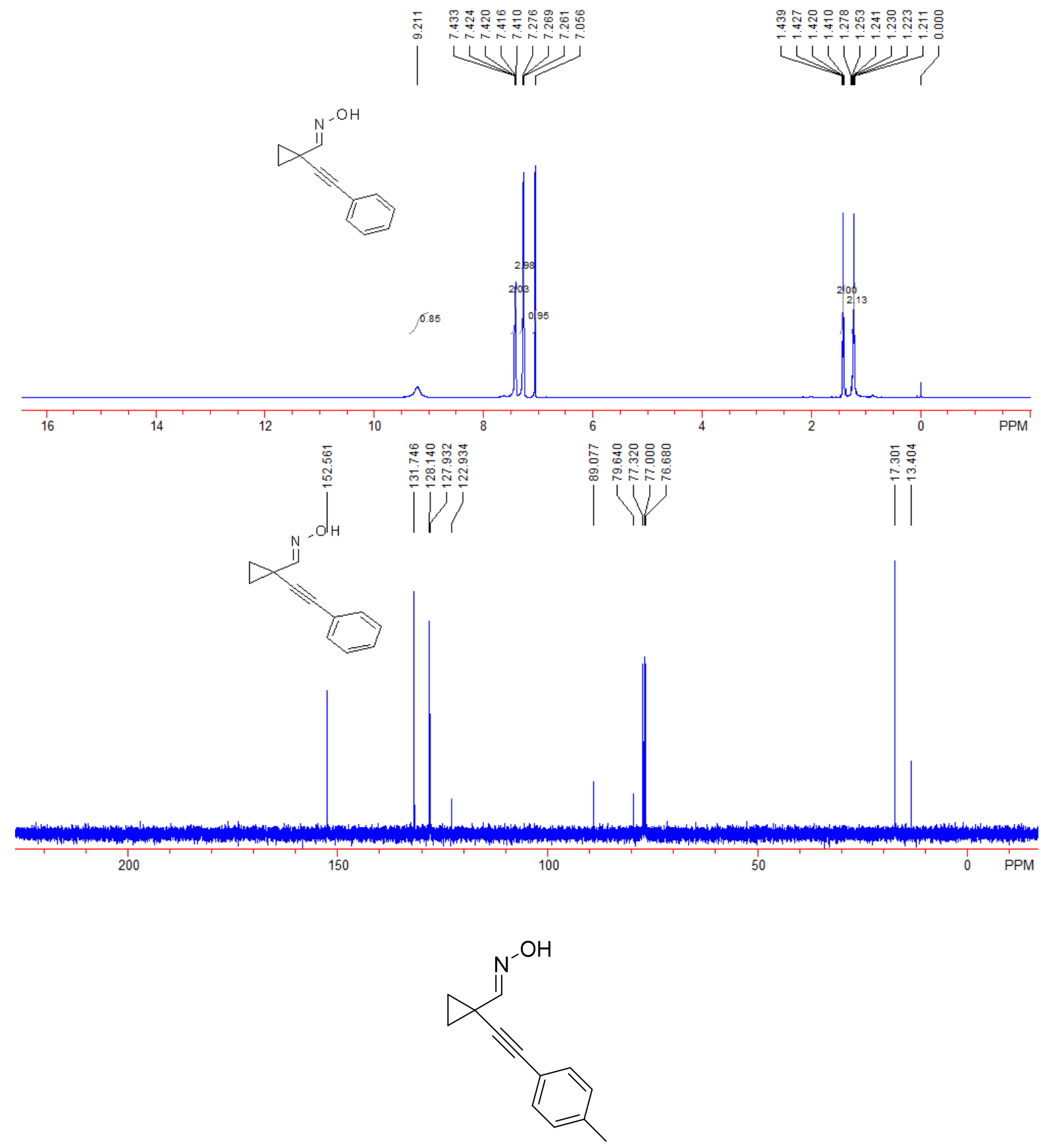

(E)-1-(p-tolylethynyl)cyclopropane-1-carbaldehyde oxime 4b: Yield: $174 \mathrm{mg}, 87 \%$; A white solid, Mp: $111-113{ }^{\circ} \mathrm{C} ;{ }^{1} \mathrm{H}$ NMR $\left(\mathrm{CDCl}_{3}, 400 \mathrm{MHz}, \mathrm{TMS}\right) \delta 1.21-1.24(\mathrm{~m}, 2 \mathrm{H}, 2 \mathrm{CH}), 1.39-1.42$ (m, 2H, 2CH), 2.32 (s, 3H, $\mathrm{CH}_{3}$ ), 7.07 (s, 1H, CH), 7.09 (d, J=4.0 Hz, 2H, Ar), 7.31 (d, J=4.0 Hz, 2H, Ar), 8.62 (br, 1H, OH). ${ }^{13} \mathrm{C} \mathrm{NMR}\left(\mathrm{CDCl}_{3}, 100 \mathrm{MHz}, \mathrm{TMS}\right) \delta 13.4,17.2,21.4,79.8,88.2,119.8,128.9$, 131.6, 137.9, 152.5. IR (neat) v 3214, 3147, 2918, 1663, 1510, 1455, 1427, 1303, 1236, 1066, 1037, 992, 944, 914, 851, 815, $732 \mathrm{~cm}^{-1}$. MS (ESI) $\mathrm{m} / z 200(\mathrm{M}+\mathrm{H})^{+}$. HRMS (ESI) calcd. for $\mathrm{C}_{13} \mathrm{H}_{14} \mathrm{NO}$ : 200.1070, Found: 200.1079. 

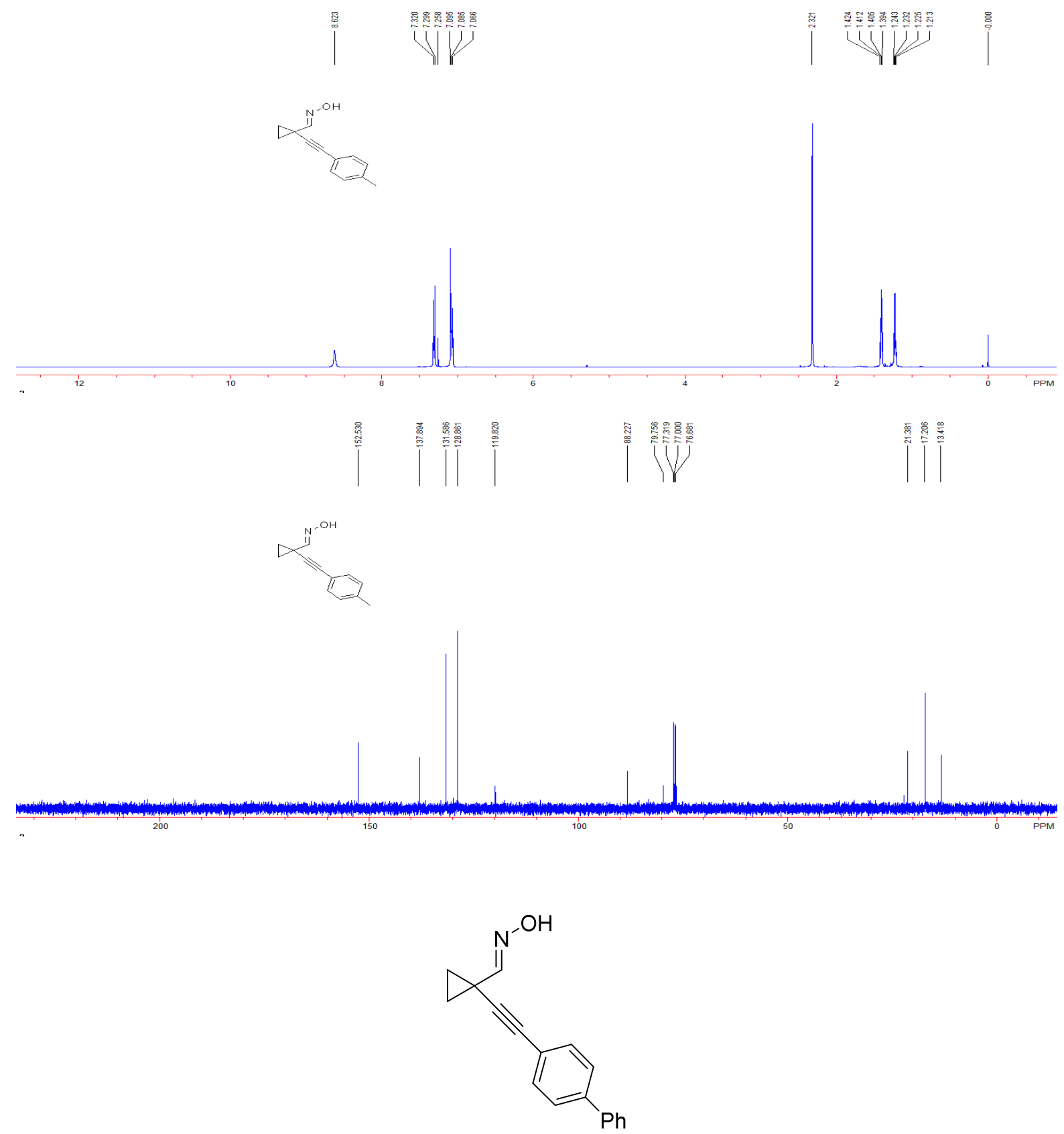

(E)-1-([1,1'-biphenyl]-4-ylethynyl)cyclopropane-1-carbaldehyde oxime 4c: Yield: 256 mg, 98\%; A white solid, Mp: $127-129{ }^{\circ} \mathrm{C} ;{ }^{1} \mathrm{H}$ NMR $\left(\mathrm{CDCl}_{3}, 400 \mathrm{MHz}, \mathrm{TMS}\right) \delta$ 1.24-1.28 (m, 2H, 2CH), 1.43-1.46 (m, 2H, 2CH), 7.11 (s, 1H, CH), 7.32-7.36 (m, 1H, Ar), 7.41-7.45 (m, 2H, Ar), 7.48-7.53 (m, 4H, Ar), 7.57 (d, $J=8.4 \mathrm{~Hz}, 2 \mathrm{H}, \mathrm{Ar}), 8.75$ (br, 1H, OH). ${ }^{13} \mathrm{C} \mathrm{NMR}\left(\mathrm{CDCl}_{3}, 100 \mathrm{MHz}, \mathrm{TMS}\right)$ $\delta 13.5,17.4,79.5,89.8,121.9,126.8,127.0,128.8,132.2,140.4,140.6,152.6$. IR (neat) $v 3243$, 3028, 2921, 2360, 2341, 1666, 1601, 1518, 1486, 1445, 1429, 1404, 1351, 1302, 1059, 1034, 988, 938, 917, 843, 760, 725, 696, 668, $653 \mathrm{~cm}^{-1}$. MS (ESI) $\mathrm{m} / z 262(\mathrm{M}+\mathrm{H})^{+}$. HRMS (ESI) calcd. for $\mathrm{C}_{18} \mathrm{H}_{16} \mathrm{NO}$ : 262.1226, Found: 262.1229. 

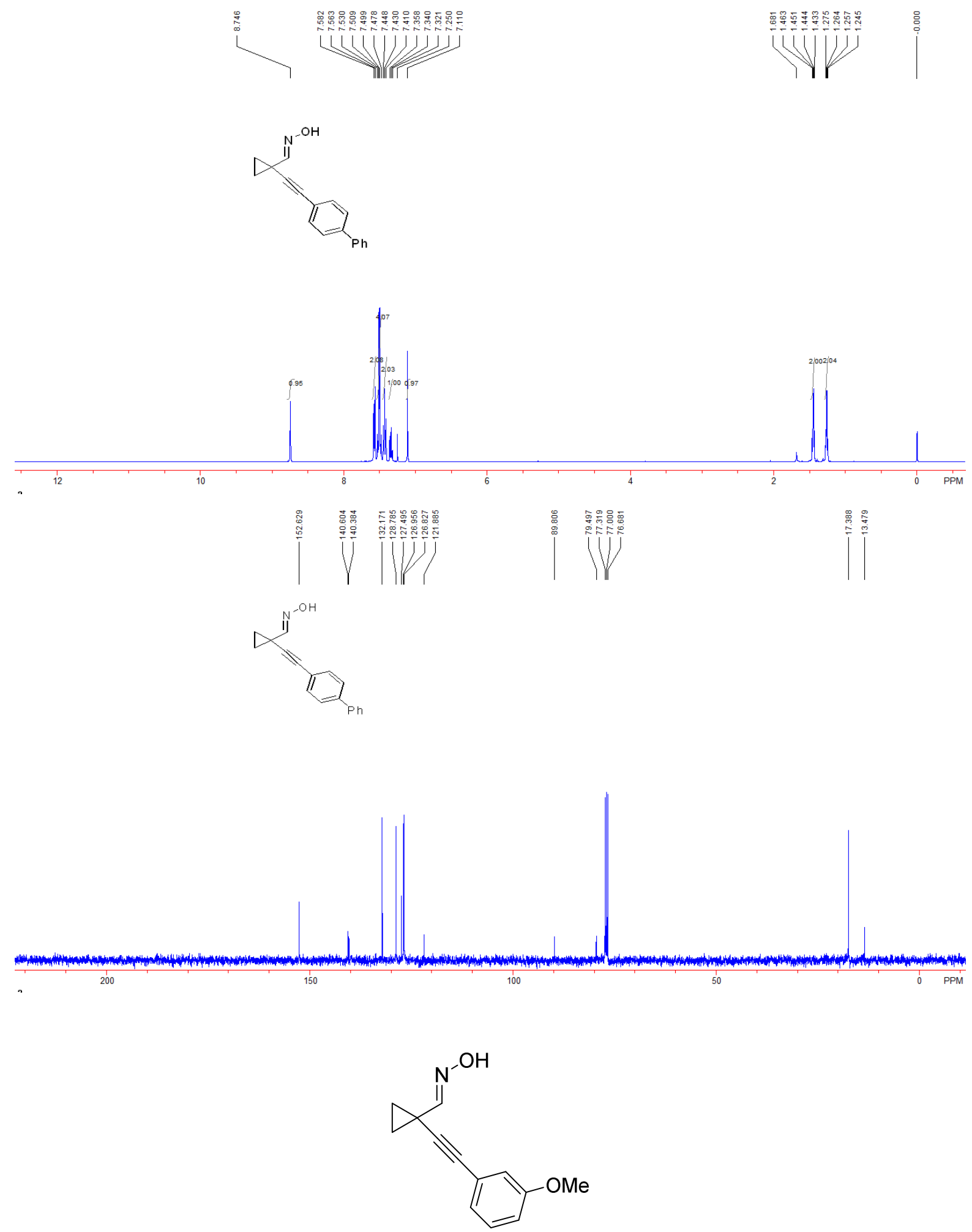

(E)-1-((3-methoxyphenyl)ethynyl)cyclopropane-1-carbaldehyde oxime 4d: Yield: $194 \mathrm{mg}$, 90\%; A yellow solid, Mp: $118-120{ }^{\circ} \mathrm{C} ;{ }^{1} \mathrm{H}$ NMR $\left(\mathrm{CDCl}_{3}, 400 \mathrm{MHz}, \mathrm{TMS}\right) \delta 1.22-1.25(\mathrm{~m}, 2 \mathrm{H}, 2 \mathrm{CH})$, 1.42-1.45 (m, 2H, 2CH), 3.79 (s, 3H, $\left.\mathrm{CH}_{3}\right), 6.82-6.85$ (m, 1H, Ar), 6.96-6.97 (m, 1H, Ar), 7.00-7.02 (m, 1H, Ar), $7.06(\mathrm{br}, 1 \mathrm{H}, \mathrm{CH}), 7.18\left(\mathrm{dd}, J_{l}=8.0 \mathrm{~Hz}, J_{2}=8.0 \mathrm{~Hz}, 1 \mathrm{H}, \mathrm{Ar}\right), 9.11(\mathrm{br}, 1 \mathrm{H}, \mathrm{OH}) .{ }^{13} \mathrm{C}$ 
NMR $\left(\mathrm{CDCl}_{3}, 100 \mathrm{MHz}, \mathrm{TMS}\right) \delta 13.4,17.3,55.2,79.7,88.9,114.8,116.4,123.9,124.2,129.2$, 152.4, 159.1. IR (neat) v 3220, 3013, 2974, 2943, 2920, 2827, 2225, 1592, 1579, 1489, 1469, 1445, $1420,1321,1307,1278,1257,1234,1165,1065,1036,997,956,930,905,844,793,741,683,671$ $\mathrm{cm}^{-1}$. MS (ESI) $m / z 216(\mathrm{M}+\mathrm{H})^{+}$. HRMS (ESI) calcd. for $\mathrm{C}_{13} \mathrm{H}_{14} \mathrm{NO}_{2}$ : 216.1019, Found: 216.1020 .
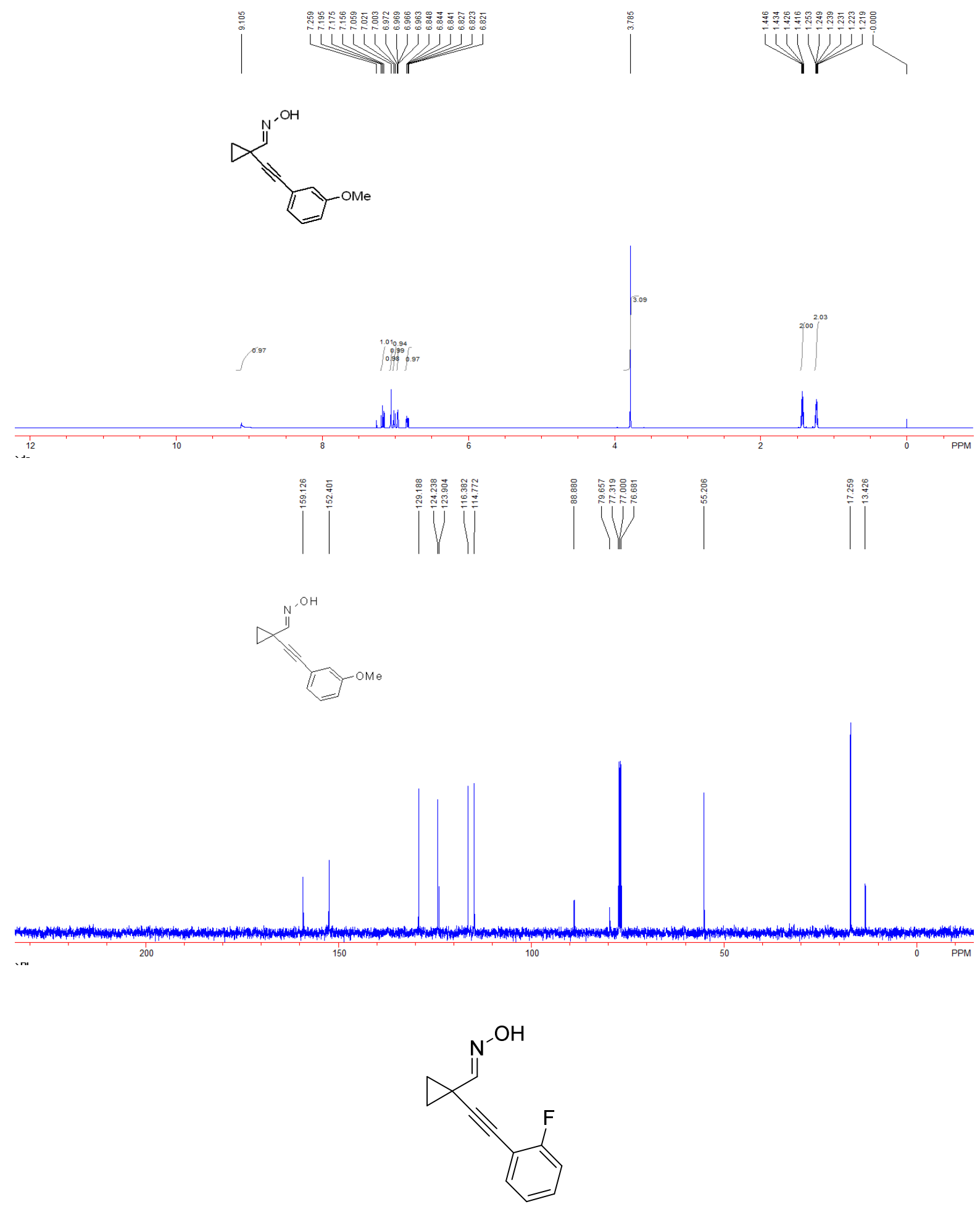
(E)-1-((2-fluorophenyl)ethynyl)cyclopropane-1-carbaldehyde oxime 4e: Yield: $198 \mathrm{mg}, 96 \%$; A yellow solid, Mp: 117-119 ${ }^{\circ} \mathrm{C} ;{ }^{1} \mathrm{H}$ NMR $\left(\mathrm{CDCl}_{3}, 400 \mathrm{MHz}, \mathrm{TMS}\right) \delta$ 1.25-1.28 (m, 2H, 2CH), 1.45-1.48 (m, 2H, 2CH), 7.00-7.08 (m, 3H, 2Ar, 1CH), 7.22-7.27 (m, 1H, Ar), 7.42-7.46 (m, 1H,

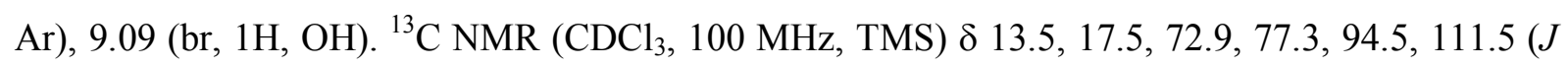
$=15.2 \mathrm{~Hz}), 115.2(J=20.5 \mathrm{~Hz}), 123.8(J=3.8 \mathrm{~Hz}), 129.6(J=8.4 \mathrm{~Hz}), 133.8,152.2,162.8(J=$ $249.8 \mathrm{~Hz}) .{ }^{19} \mathrm{~F}$ NMR (376 MHz, $\left.\mathrm{CDCl}_{3}, \mathrm{CFCl}_{3}\right) \delta$-110.37-(-110.42) (m). IR (neat) $v$ 3269, 2361, 2324, 1572, 1493, 1448, 1428, 1295, 1265, 1238, 1223, 1103, 1069, 1043, 991, 939, 922, 860, 799, 759, $722 \mathrm{~cm}^{-1}$. MS (ESI) $\mathrm{m} / z 204(\mathrm{M}+\mathrm{H})^{+}$. HRMS (ESI) calcd. for $\mathrm{C}_{12} \mathrm{H}_{11} \mathrm{FNO}$ : 204.0819, Found: 204.0821.
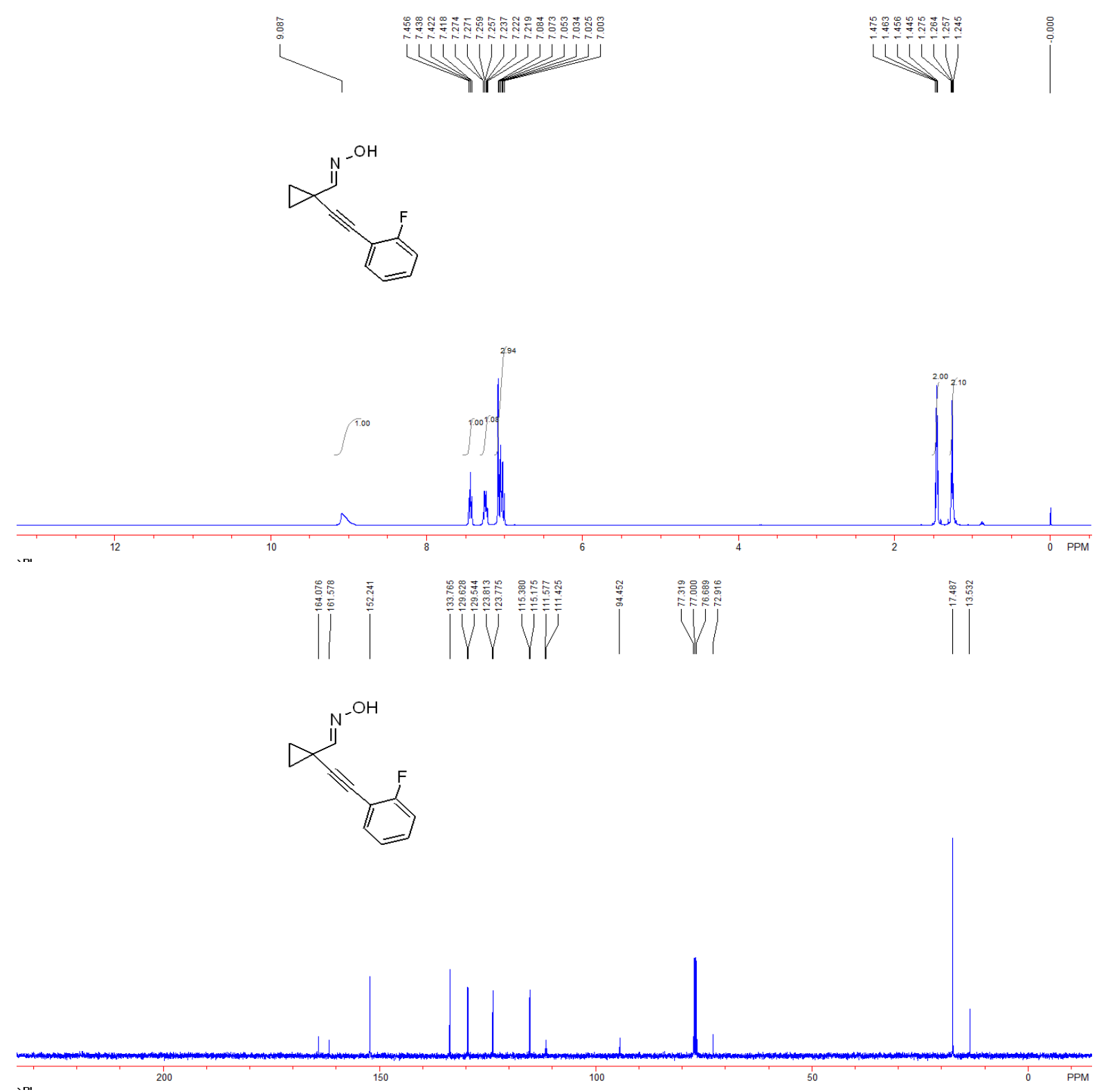


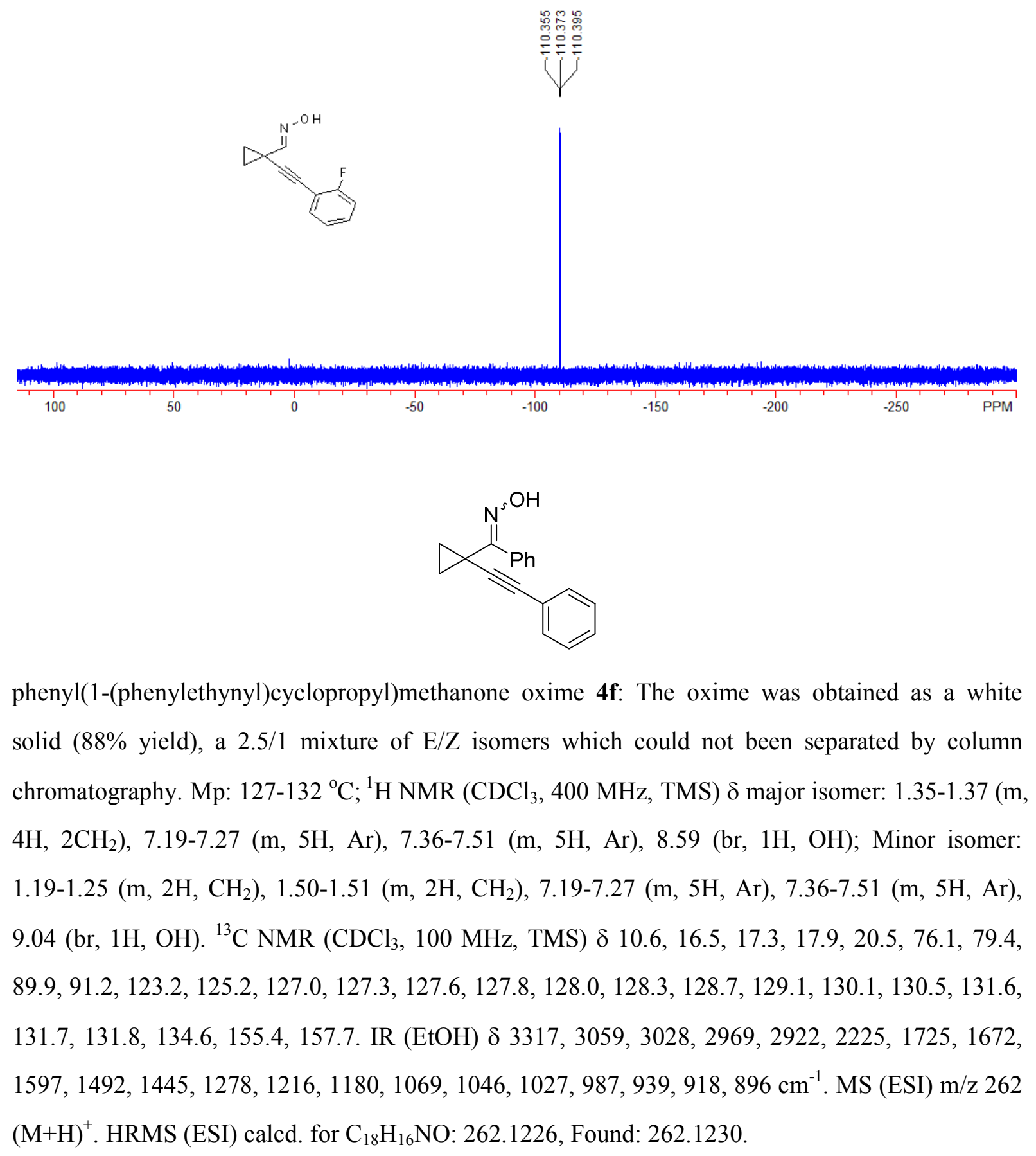



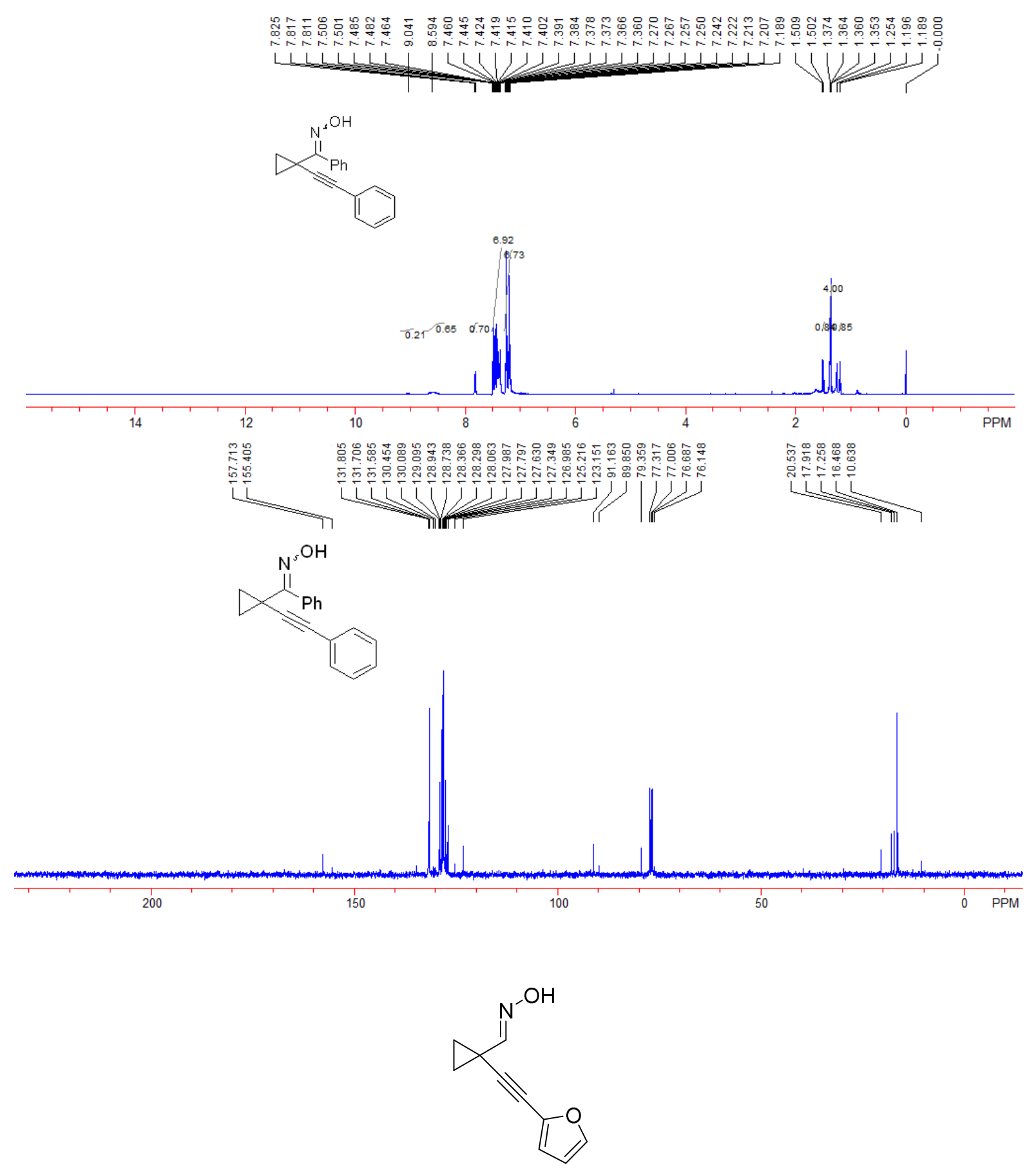

(E)-1-(furan-2-ylethynyl)cyclopropane-1-carbaldehyde oxime 4g: Yield: $170 \mathrm{mg}$, 98\%; A yellow solid, Mp: $92-94{ }^{\circ} \mathrm{C} ;{ }^{1} \mathrm{H}$ NMR $\left(\mathrm{CDCl}_{3}, 400 \mathrm{MHz}\right.$, TMS) $\delta$ 1.25-1.28 (m, 2H, 2CH), 1.43-1.46 (m, 2H, 2CH), 6.34-6.35 (m, 1H, Ar), 6.56-6.57 (m, 1H, Ar), 7.08 (s, 1H, CH), 7.33-7.34 (m, 1H, Ar), 8.62 (br, $1 \mathrm{H}, \mathrm{OH}) .{ }^{13} \mathrm{C} \mathrm{NMR}\left(\mathrm{CDCl}_{3}, 100 \mathrm{MHz}, \mathrm{TMS}\right) \delta$ 13.3, 17.5, 69.8, 93.6, 110.7, 115.2, 136.7, 143.2, 152.0. IR $(\mathrm{EtOH}) \vee 3243,3153,2956,2922,2850,2230,1724,1660,1573,1488,1455$, $1427,1307,1222,1203,1150,1071,1040,990,967,948,915,883,874,824,812,789,731 \mathrm{~cm}^{-1}$. 
MS (EI) m/z 175 (M+, 33), 158 (100), 77 (61), 103 (55), 131 (44), 175 (33), 130 (31), 149 (22). HRMS (EI) calcd. for $\mathrm{C}_{10} \mathrm{H}_{9} \mathrm{NOS}$ : 175.0633, Found: 175.0629.
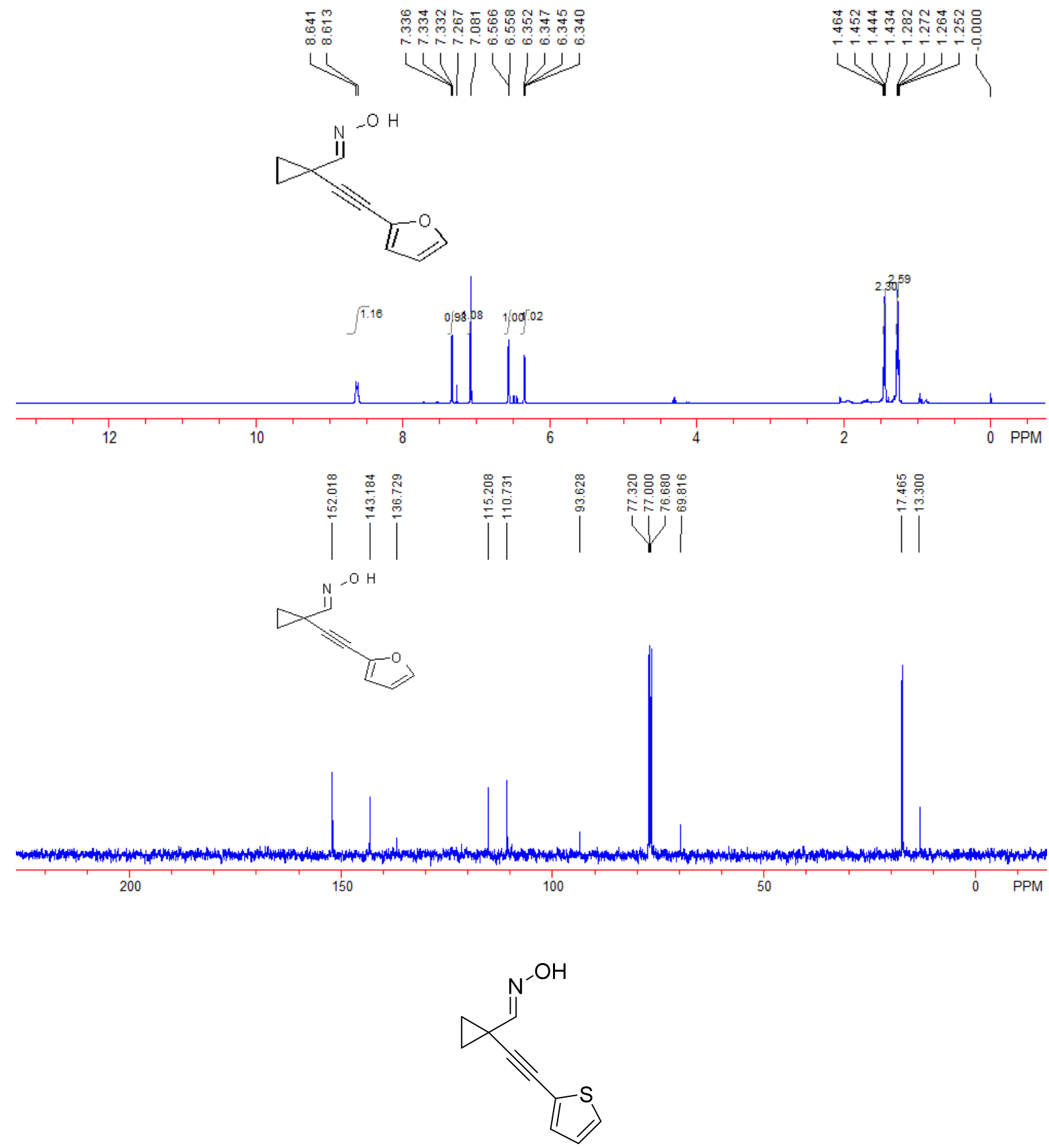

(E)-1-(thiophen-2-ylethynyl)cyclopropane-1-carbaldehyde oxime 4h: Yield: $170 \mathrm{mg}$, 89\%; A yellow solid, Mp: 94-96 ${ }^{\circ} \mathrm{C} ;{ }^{1} \mathrm{H}$ NMR $\left(\mathrm{CDCl}_{3}, 400 \mathrm{MHz}, \mathrm{TMS}\right) \delta 1.25-1.28(\mathrm{~m}, 2 \mathrm{H}, 2 \mathrm{CH})$, 1.42-1.45 (m, 4H, 2CH), 6.92-6.94 (m, 1H, Ar), 7.11 (s, 1H, CH), 7.19-7.20 (m, 2H, Ar), 8.31 (br, $1 \mathrm{H}, \mathrm{OH}) .{ }^{13} \mathrm{C} \mathrm{NMR}\left(\mathrm{CDCl}_{3}, 100 \mathrm{MHz}, \mathrm{TMS}\right) \delta 13.5,17.5,72.7,93.1,123.0,126.8,132.2,152.5$. IR $(\mathrm{EtOH}) \vee 3308,3103,2953,2922,2851,1732,1714,1646,1463,1325,1163,1125,1066,1018$, 
984, 953, 932, $852 \mathrm{~cm}^{-1}$. MS (EI) m/z 191 (M+, 32), 174 (100), 147 (53), 173 (40), 191 (32), 175 (18), 121 (16), 172 (12), 69 (12). HRMS (EI) calcd. for $\mathrm{C}_{10} \mathrm{H}_{9} \mathrm{NOS}: 191.0405$, Found: 191.0399.

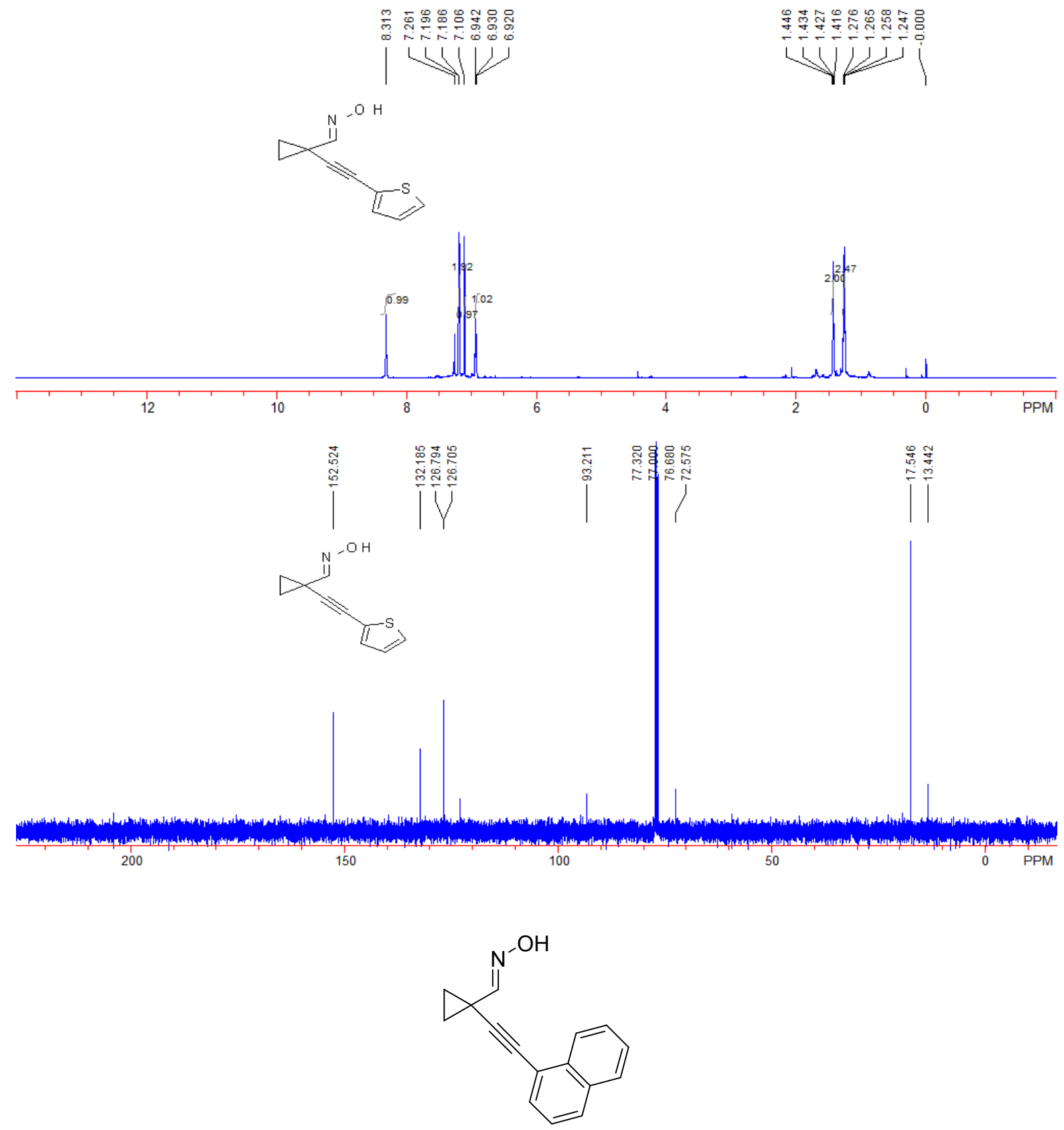

(E)-1-(naphthalen-1-ylethynyl)cyclopropane-1-carbaldehyde oxime 4i: Yield: $236 \mathrm{mg}$, 99\%; A yellow solid, Mp: $130-132{ }^{\circ} \mathrm{C} ;{ }^{1} \mathrm{H}$ NMR $\left(\mathrm{CDCl}_{3}, 400 \mathrm{MHz}, \mathrm{TMS}\right) \delta 1.29-1.32(\mathrm{~m}, 2 \mathrm{H}, 2 \mathrm{CH})$, 1.50-1.53 (m, 2H, 2CH), $7.16(\mathrm{~s}, 1 \mathrm{H}, \mathrm{CH}), 7.38\left(\mathrm{dd}, J_{1}=7.2 \mathrm{~Hz}, J_{2}=8.4 \mathrm{~Hz}, 1 \mathrm{H}, \mathrm{Ar}\right), 7.48-7.50$ (m, $1 \mathrm{H}, \mathrm{Ar}), 7.54-7.56(\mathrm{~m}, 1 \mathrm{H}, \mathrm{Ar}), 7.66\left(\mathrm{dd}, J_{1}=1.2 \mathrm{~Hz}, J_{2}=8.0 \mathrm{~Hz}, 1 \mathrm{H}, \mathrm{Ar}\right), 7.77(\mathrm{~d}, J=8.0 \mathrm{~Hz}, 1 \mathrm{H}$, Ar), $7.80(\mathrm{~d}, J=8.4 \mathrm{~Hz}, 1 \mathrm{H}, \mathrm{Ar}), 8.27$ (d, $J=8.4 \mathrm{~Hz}, 1 \mathrm{H}, \mathrm{Ar}), 8.88$ (br, $1 \mathrm{H}, \mathrm{OH}) .{ }^{13} \mathrm{C} \mathrm{NMR}\left(\mathrm{CDCl}_{3}\right.$, $100 \mathrm{MHz}, \mathrm{TMS}) \delta 13.6,17.7,77.6,94.2,120.6,125.2,126.1,126.2,126.6,128.2,128.4,130.5$, 
133.0, 133.4, 152.6. IR (neat) v 3221, 3017, 2970, 2225, 1944, 1865, 1662, 1592, 1579, 1488, 1469, 1446, 1429, 1420, 1359, 1321, 1277, 1257, 1234, 1192, 1165, 1065, 1036, 997, 956, 930, 905, 844, 792, 740, 683, $671 \mathrm{~cm}^{-1}$. MS (ESI) $\mathrm{m} / z 236(\mathrm{M}+\mathrm{H})^{+}$. HRMS (ESI) calcd. for $\mathrm{C}_{16} \mathrm{H}_{14} \mathrm{NO}$ : 236.1070, Found: 236.1071.
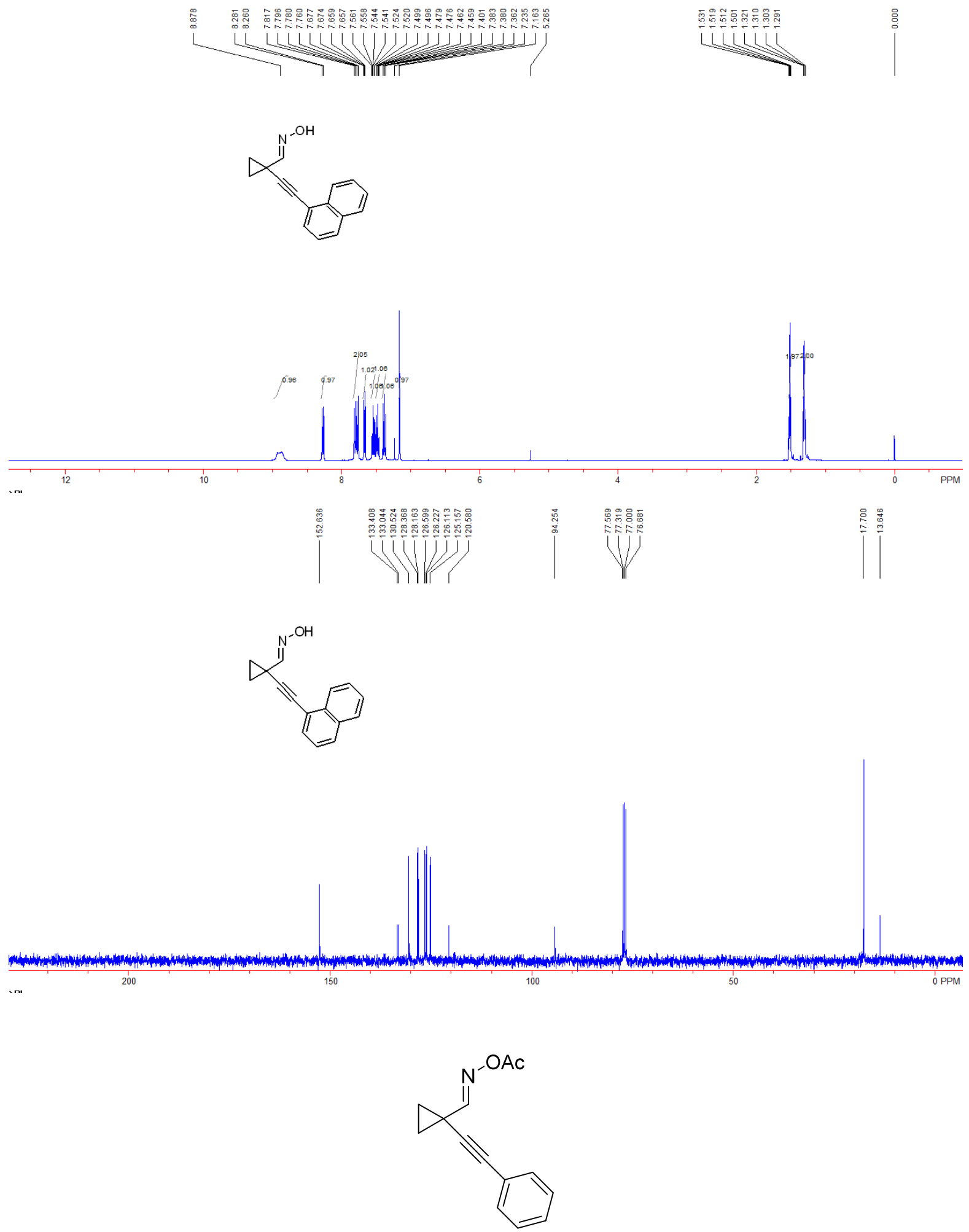
(E)-1-(phenylethynyl)cyclopropane-1-carbaldehyde O-acetyl oxime 7a: Yield: $44.0 \mathrm{mg}$, 96\%; A colorless oil; ${ }^{1} \mathrm{H}$ NMR $\left(\mathrm{CDCl}_{3}, 400 \mathrm{MHz}, \mathrm{TMS}\right) \delta 1.39$ (br, 4H, 2CH$), 2.03\left(\mathrm{~s}, 3 \mathrm{H}, \mathrm{CH}_{3}\right)$,

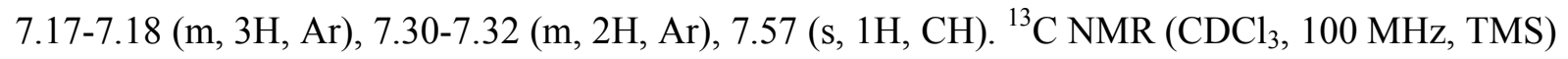
$\delta 12.9,19.16,19.21,79.0,88.6,122.5,127.90,127.97,131.5,160.1,168.3$. IR (neat) $v 3082,3054$, $3015,2938,2235,1762,1625,1490,1365,1194,1069,1040,1000,973,948,916,889,824,755$, $691 \mathrm{~cm}^{-1}$. MS (ESI) $\mathrm{m} / z 228(\mathrm{M}+\mathrm{H})^{+}$. HRMS (ESI) calcd. for $\mathrm{C}_{14} \mathrm{H}_{14} \mathrm{NO}_{2}$ : 228.1025, Found: 228.1025 .

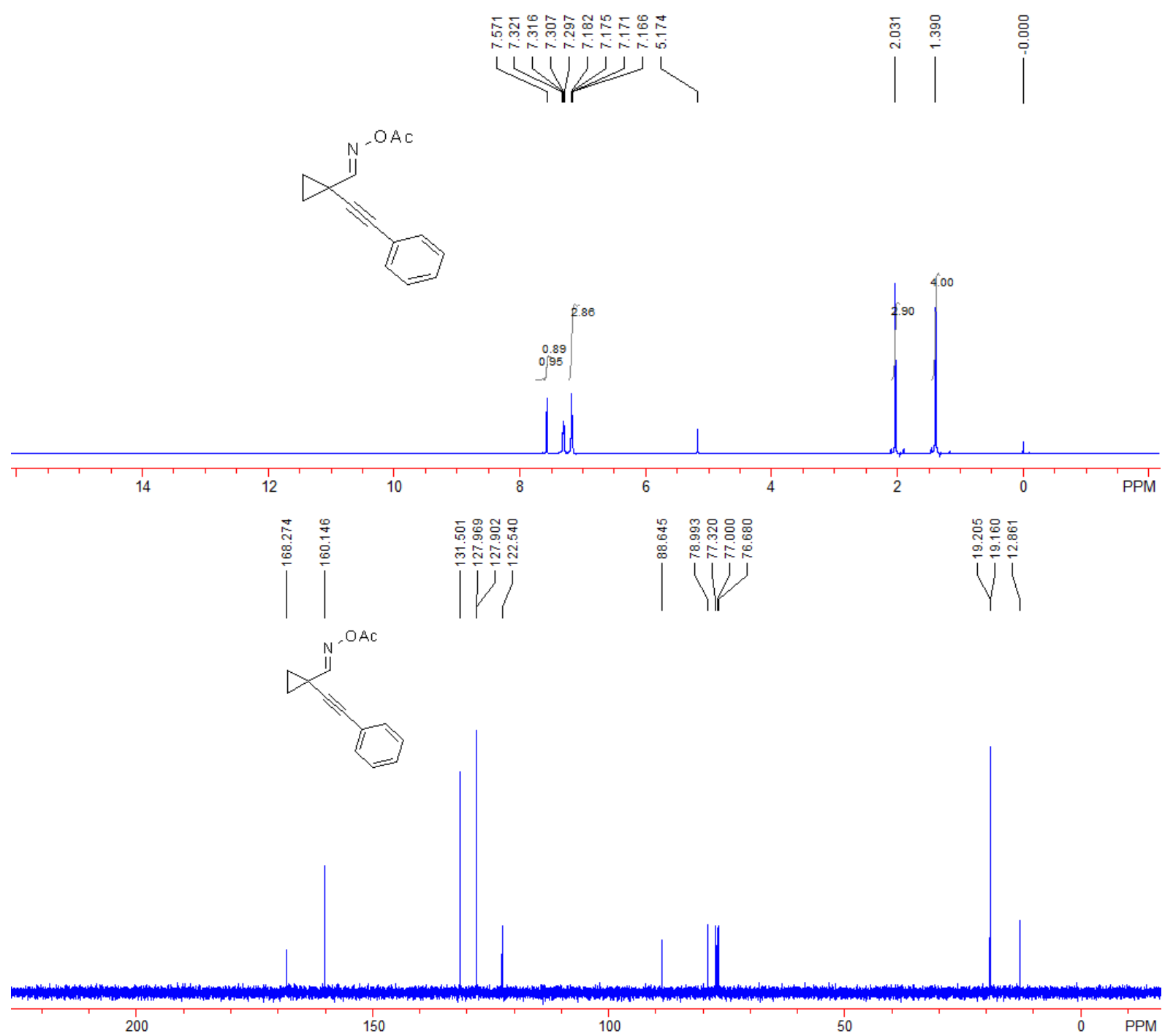




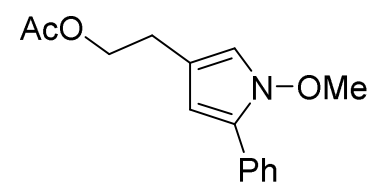

2-(1-methoxy-5-phenyl-1H-pyrrol-3-yl)ethyl acetate 2a: Yield: $48.6 \mathrm{mg}, 94 \%$; A colorless oil; ${ }^{1} \mathrm{H}$ NMR $\left(\mathrm{CDCl}_{3}, 400 \mathrm{MHz}, \mathrm{TMS}\right) \delta 2.07\left(\mathrm{~s}, 3 \mathrm{H}, \mathrm{CH}_{3}\right), 2.79\left(\mathrm{t}, J=7.2 \mathrm{~Hz}, 2 \mathrm{H}, \mathrm{CH}_{2}\right), 3.78(\mathrm{~s}, 3 \mathrm{H}$, $\left.\mathrm{CH}_{3}\right), 4.24$ (t, $\left.J=7.2 \mathrm{~Hz}, 2 \mathrm{H}, \mathrm{CH}_{2}\right), 6.11(\mathrm{~d}, J=2.0 \mathrm{~Hz}, 1 \mathrm{H}, \mathrm{Ar}), 6.74$ (d, $\left.J=2.0 \mathrm{~Hz}, 1 \mathrm{H}, \mathrm{Ar}\right)$, 7.23-7.27 (m, 1H, Ar), 7.35-7.39 (m, 2H, Ar), 7.64-7.66 (m, 2H, Ar). ${ }^{13} \mathrm{C}$ NMR $\left(\mathrm{CDCl}_{3}, 100 \mathrm{MHz}\right.$, TMS) $\delta 21.0,26.6,64.9,66.4,104.4,114.7,115.5,126.2,126.5,127.9,128.4,130.8,171.0$. IR $(\mathrm{EtOH}) \vee 3322,2940,1734,1600,1573,1509,1448,1364,1234,1032,966,759,696 \mathrm{~cm}^{-1} . \mathrm{MS}$ (ESI) $m / z 260(\mathrm{M}+\mathrm{H})^{+}$. HRMS (ESI) calcd. for $\mathrm{C}_{15} \mathrm{H}_{18} \mathrm{NO}_{3}: 260.1281$, Found: 260.1283.

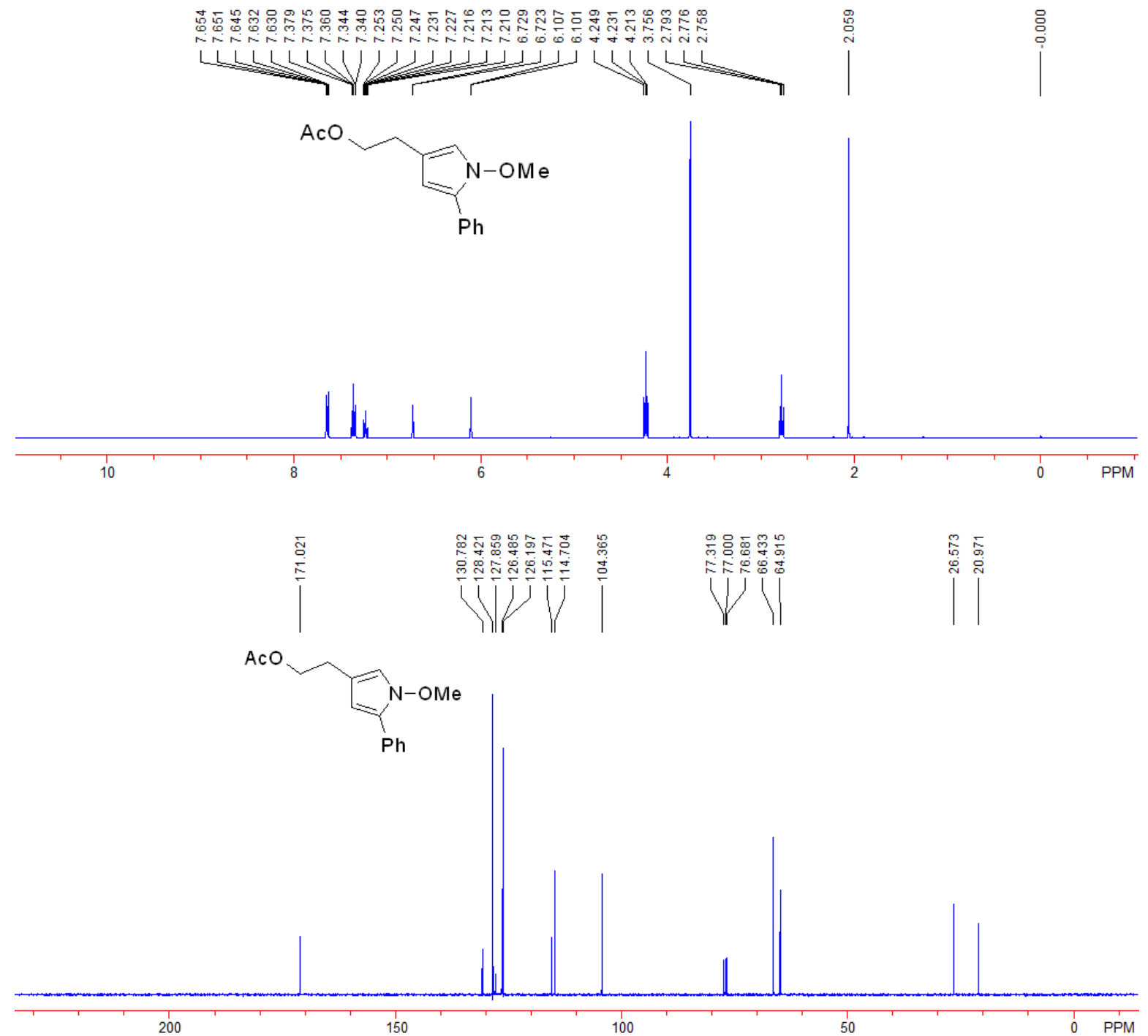

\section{DEPT135:}




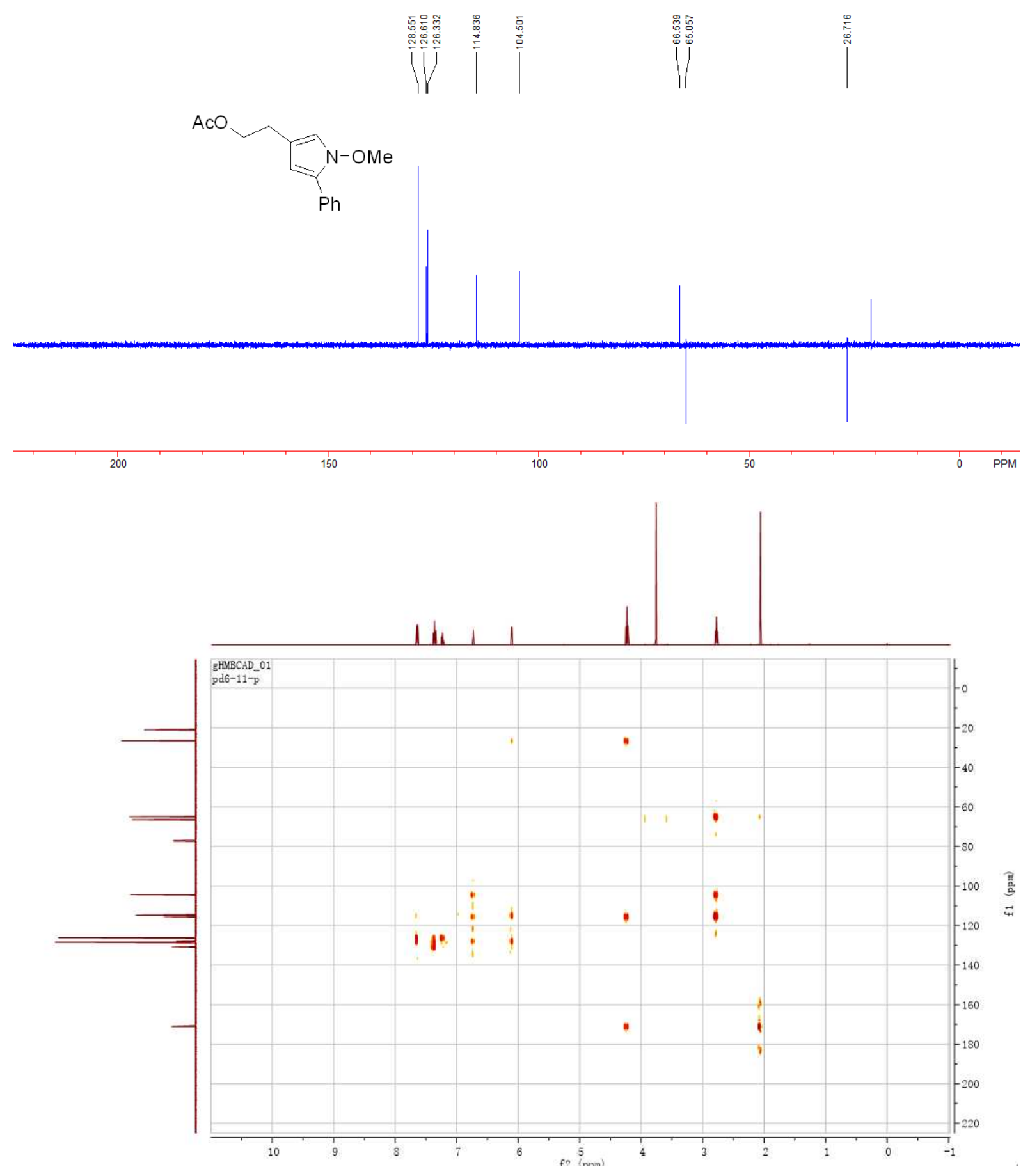



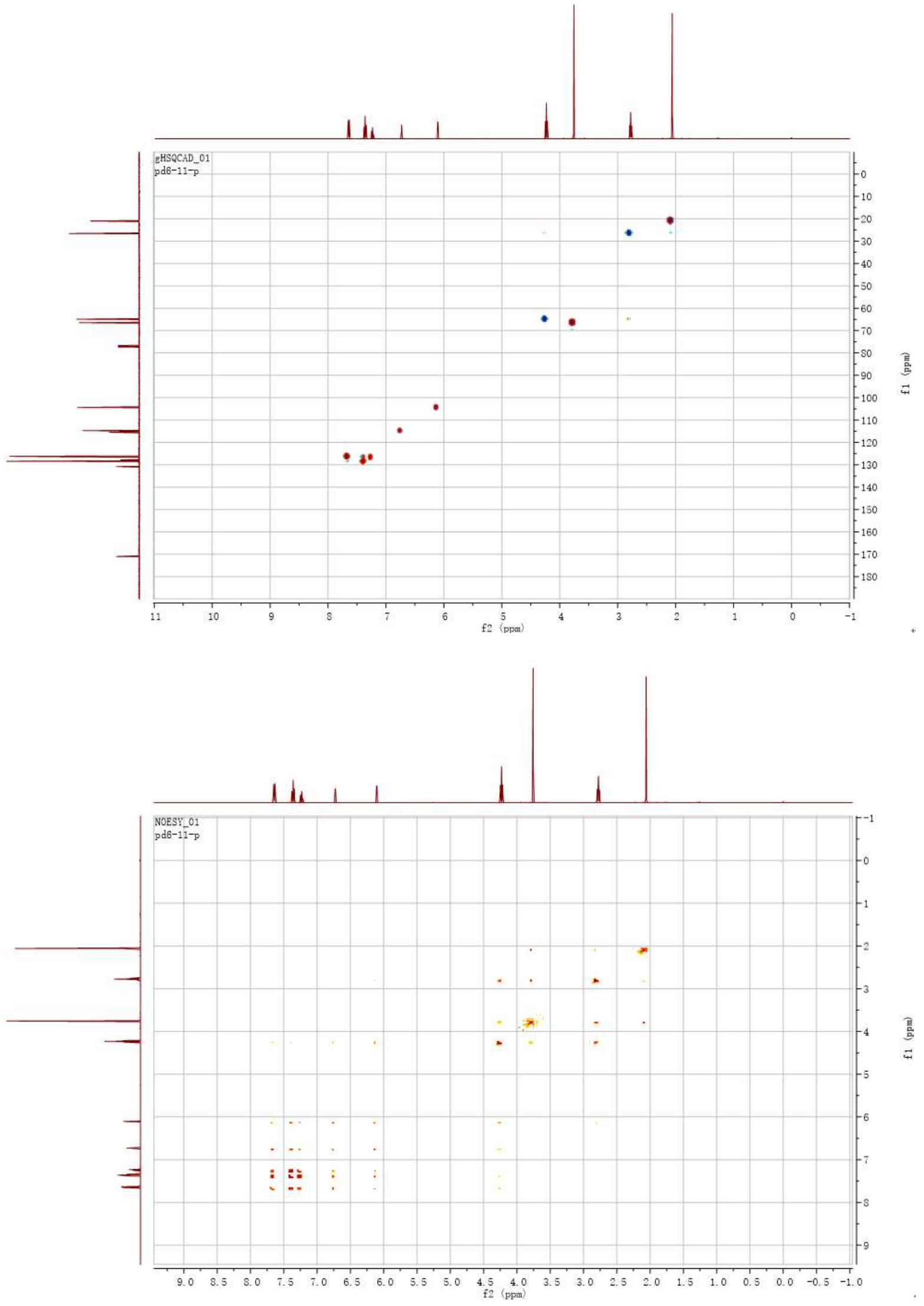

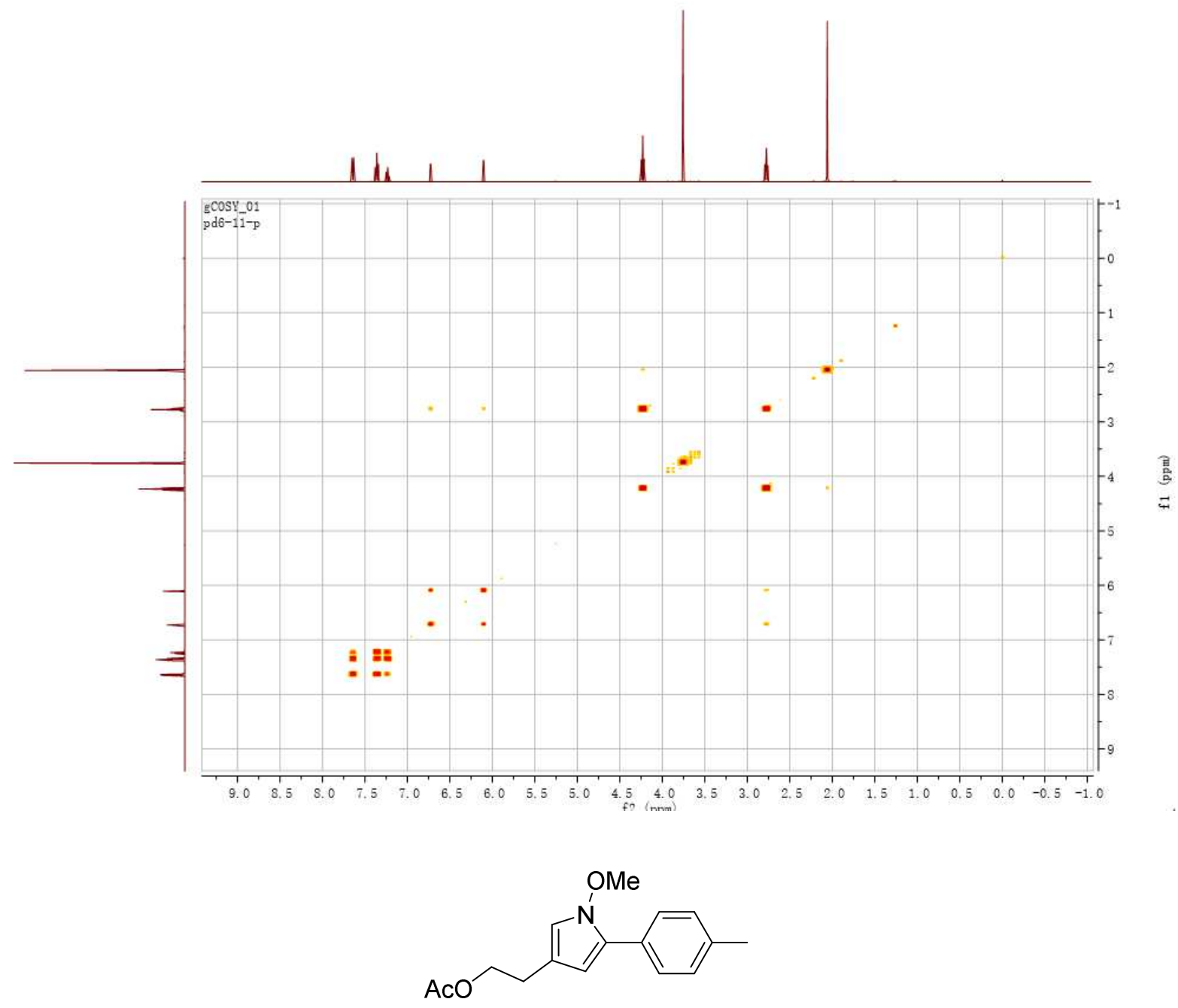

2-(1-methoxy-5-(p-tolyl)-1H-pyrrol-3-yl)ethyl acetate $\mathbf{2 b}$ : Yield: $50.1 \mathrm{mg}, 90 \%$; A colorless oil; ${ }^{1} \mathrm{H}$ NMR $\left(\mathrm{CDCl}_{3}, 400 \mathrm{MHz}, \mathrm{TMS}\right) \delta 2.07\left(\mathrm{~s}, 3 \mathrm{H}, \mathrm{CH}_{3}\right), 2.36\left(\mathrm{~s}, 3 \mathrm{H}, \mathrm{CH}_{3}\right), 2.78(\mathrm{t}, J=7.2 \mathrm{~Hz}, 2 \mathrm{H}$, $\left.\mathrm{CH}_{2}\right), 3.76\left(\mathrm{~s}, 3 \mathrm{H}, \mathrm{CH}_{3}\right), 4.23\left(\mathrm{t}, J=7.2 \mathrm{~Hz}, 2 \mathrm{H}, \mathrm{CH}_{2}\right), 6.06$ (d, $\left.J=2.0 \mathrm{~Hz}, 1 \mathrm{H}, \mathrm{Ar}\right), 6.71$ (d, $J=2.0$ $\mathrm{Hz}, 1 \mathrm{H}, \mathrm{Ar}), 7.18$ (d, $J=8.0 \mathrm{~Hz}, 2 \mathrm{H}, \mathrm{Ar}), 7.53$ (d, $J=8.0 \mathrm{~Hz}, 2 \mathrm{H}, \mathrm{Ar}) .{ }^{13} \mathrm{C} \mathrm{NMR}\left(\mathrm{CDCl}_{3}, 100 \mathrm{MHz}\right.$, TMS) $\delta$ 21.0, 21.1, 26.6, 65.0, 66.4, 103.9, 114.3, 115.4, 126.2, 128.02, 128.04, 129.2, 136.3, 171.1. IR $(\mathrm{EtOH}) \vee 2935,1735,1576,1522,1439,1363,1235,1109,1032,1000,967,820,789,668 \mathrm{~cm}^{-1}$. MS (ESI) $m / z 274(\mathrm{M}+\mathrm{H})^{+}$. HRMS (ESI) calcd. for $\mathrm{C}_{16} \mathrm{H}_{20} \mathrm{NO}_{3}$ : 274.1438, Found: 274.1439. 


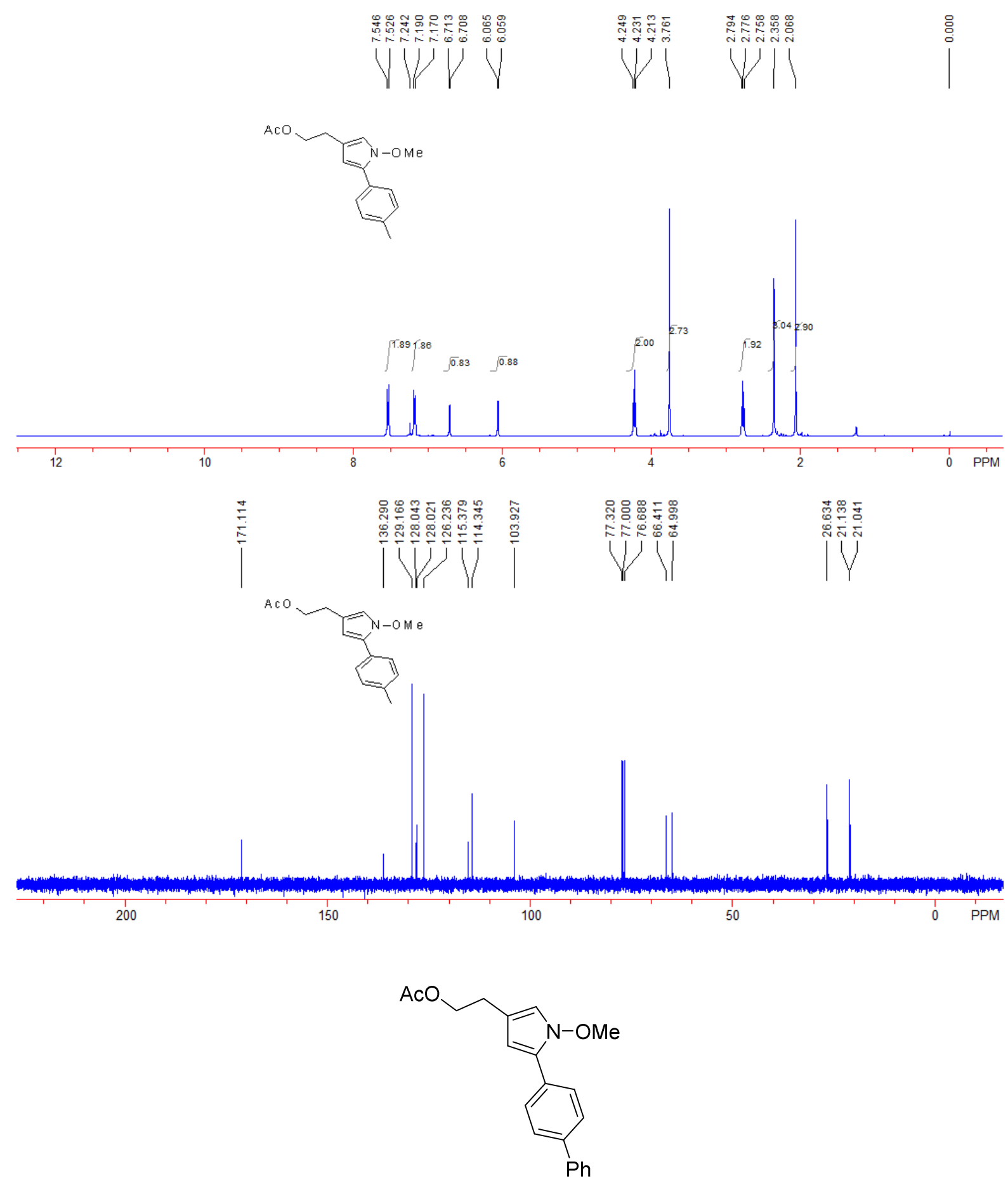

2-(5-([1,1'-biphenyl]-4-yl)-1-methoxy-1H-pyrrol-3-yl)ethyl acetate 2c: Yield: $57.6 \mathrm{mg}, 86 \%$; A colorless oil; ${ }^{1} \mathrm{H}$ NMR $\left(\mathrm{CDCl}_{3}, 400 \mathrm{MHz}, \mathrm{TMS}\right) \delta 2.07\left(\mathrm{~s}, 3 \mathrm{H}, \mathrm{CH}_{3}\right), 2.79\left(\mathrm{t}, J=7.2 \mathrm{~Hz}, 2 \mathrm{H}, \mathrm{CH}_{2}\right)$, $3.80\left(\mathrm{~s}, 3 \mathrm{H}, \mathrm{CH}_{3}\right), 4.24$ (t, $\left.J=7.2 \mathrm{~Hz}, 2 \mathrm{H}, \mathrm{CH}_{2}\right), 6.16$ (d, $\left.J=2.4 \mathrm{~Hz}, 1 \mathrm{H}, \mathrm{Ar}\right), 6.75$ (d, $J=2.4 \mathrm{~Hz}$, 1H, Ar), 7.33-7.35 (m, 1H, Ar), 7.42-7.45 (m, 2H, Ar), 7.60-7.63 (m, 4H, Ar), 7.72 (d, J=8.0 Hz, $2 \mathrm{H}, \mathrm{Ar}) .{ }^{13} \mathrm{C} \mathrm{NMR} \delta\left(\mathrm{CDCl}_{3}, 100 \mathrm{MHz}, \mathrm{TMS}\right) 21.0,26.6,65.0,66.6,104.5,115.0,115.6,126.5$, 
126.8, 127.1, 127.2, 127.5, 128.7, 129.8, 139.0, 140.6, 171.1. IR (EtOH) v 3064, 2948, 2896, 1712, 1577, 1449, 1365, 1233, 1034, 762, $698 \mathrm{~cm}^{-1}$. MS (ESI) m/z $336(\mathrm{M}+\mathrm{H})^{+}$. HRMS (ESI) calcd. for $\mathrm{C}_{21} \mathrm{H}_{22} \mathrm{NO}_{3}$ : 336.1594, Found: 336.1593.

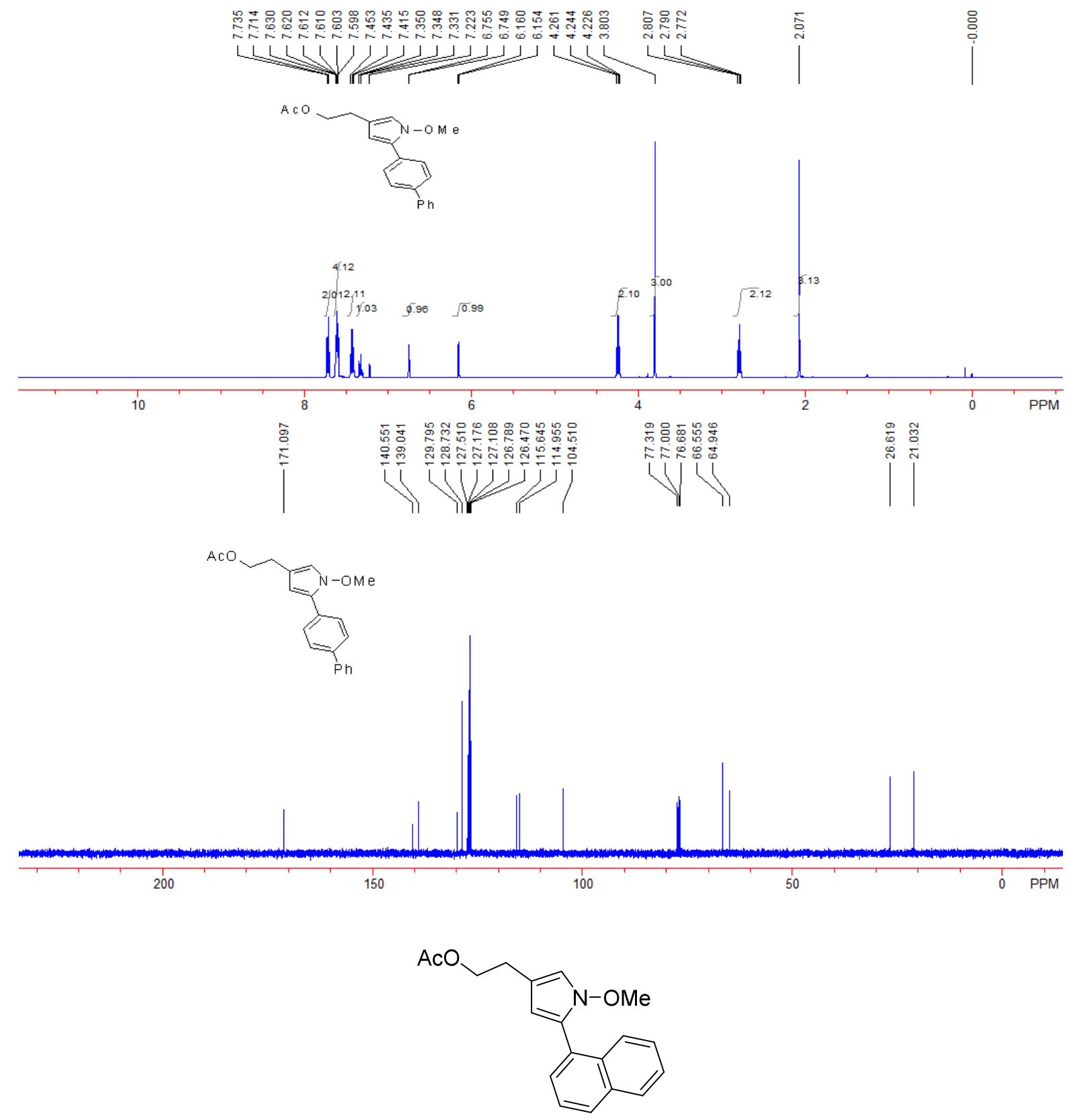

2-(1-methoxy-5-(naphthalen-1-yl)-1H-pyrrol-3-yl)ethyl acetate 2d: Yield: $58.1 \mathrm{mg}$, 94\%; A colorless oil; ${ }^{1} \mathrm{H}$ NMR $\left(\mathrm{CDCl}_{3}, 400 \mathrm{MHz}, \mathrm{TMS}\right) \delta 2.08\left(\mathrm{~s}, 3 \mathrm{H}, \mathrm{CH}_{3}\right), 2.86\left(\mathrm{t}, J=7.2 \mathrm{~Hz}, 2 \mathrm{H}, \mathrm{CH}_{2}\right)$, $3.57\left(\mathrm{~s}, 3 \mathrm{H}, \mathrm{CH}_{3}\right), 4.29$ (t, $\left.J=7.2 \mathrm{~Hz}, 2 \mathrm{H}, \mathrm{CH}_{2}\right), 6.07$ (d, $\left.J=2.0 \mathrm{~Hz}, 1 \mathrm{H}, \mathrm{Ar}\right), 6.83$ (d, $J=2.0 \mathrm{~Hz}$, 1H, Ar), 7.23-7.52 (m, 3H, Ar), 7.57-7.60 (m, 1H, Ar), 7.84-7.88 (m, 2H, Ar), 8.04-8.06 (m, 1H, Ar). ${ }^{13} \mathrm{C} \mathrm{NMR}\left(\mathrm{CDCl}_{3}, 100 \mathrm{MHz}, \mathrm{TMS}\right) \delta 21.0,26.7,65.1,66.9,107.0,113.6,115.1,125.2,125.8$, 
126.1, 126.2, 126.5, 128.05, 128.07, 128.10, 128.5, 132.1, 133.7, 171.2. IR (EtOH) v 3054, 2934, 1734, 1593, 1508, 1456, 1437, 1384, 1363, 1237, 1125, 1099, 1031, 974, 951, 865, 798, 790, 776, 737, 674, $659 \mathrm{~cm}^{-1}$. MS (ESI) $\mathrm{m} / z 310(\mathrm{M}+\mathrm{H})^{+}$. HRMS (ESI) calcd. for $\mathrm{C}_{19} \mathrm{H}_{20} \mathrm{NO}_{3}: 310.1438$, Found: 310.1438.
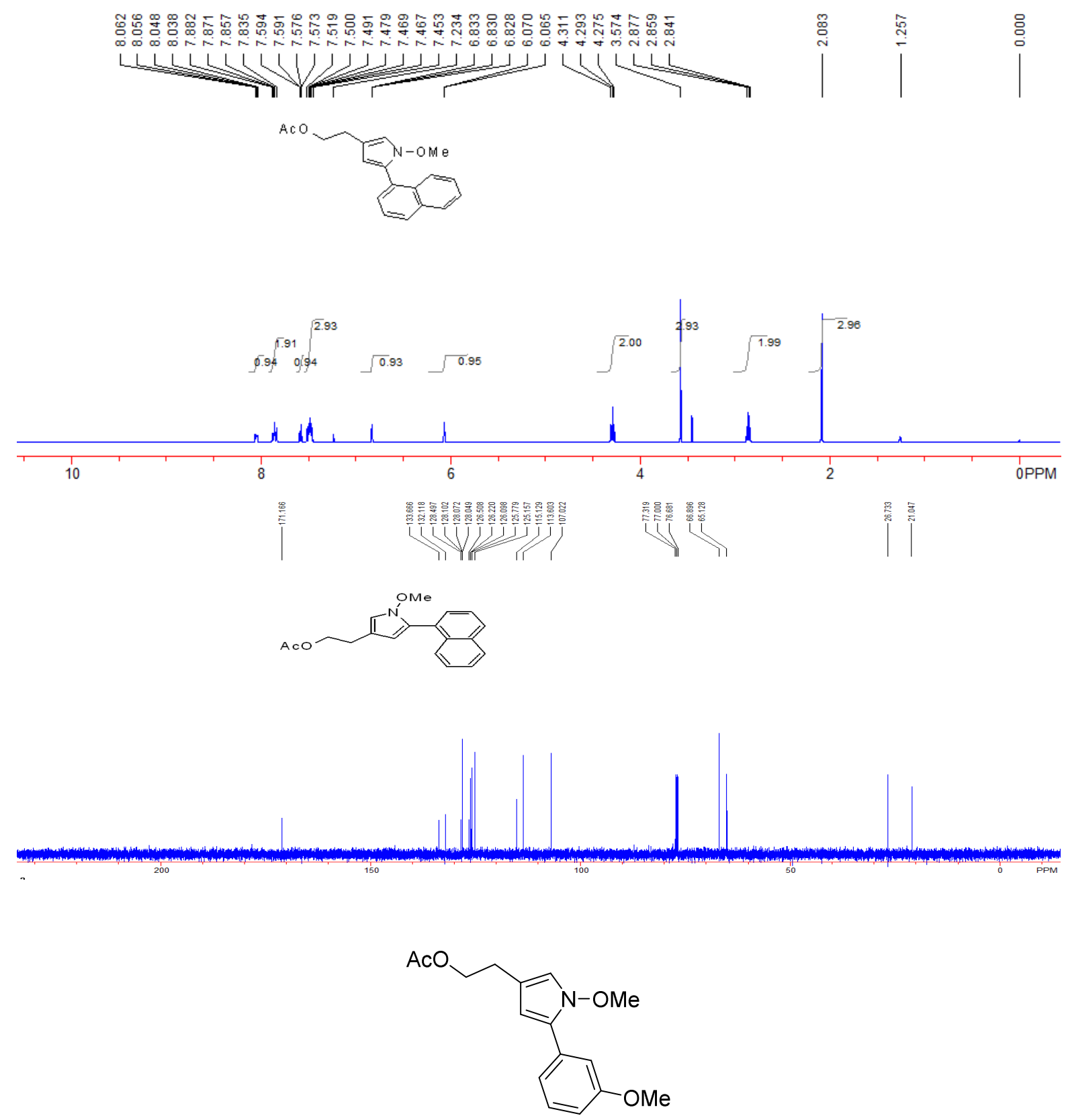

2-(1-methoxy-5-(3-methoxyphenyl)-1H-pyrrol-3-yl)ethyl acetate 2e: Yield: $54.9 \mathrm{mg}, 95 \%$; A colorless oil; ${ }^{1} \mathrm{H}$ NMR $\left(\mathrm{CDCl}_{3}, 400 \mathrm{MHz}, \mathrm{TMS}\right) \delta 2.06\left(\mathrm{~s}, 3 \mathrm{H}, \mathrm{CH}_{3}\right), 2.77$ (t, J=7.2 Hz, 2H, $\mathrm{CH}_{2}$ ), $3.78\left(\mathrm{~s}, 3 \mathrm{H}, \mathrm{CH}_{3}\right), 3.82\left(\mathrm{~s}, 3 \mathrm{H}, \mathrm{CH}_{3}\right), 4.23\left(\mathrm{t}, J=7.2 \mathrm{~Hz}, 2 \mathrm{H}, \mathrm{CH}_{2}\right), 6.11(\mathrm{~s}, 1 \mathrm{H}, \mathrm{Ar}), 6.72(\mathrm{~s}, 1 \mathrm{H}$, Ar), $6.80(\mathrm{~d}, J=8.0 \mathrm{~Hz}, 1 \mathrm{H}, \mathrm{Ar}), 7.22-7.28$ (m, 3H, $\mathrm{Ar}) .{ }^{13} \mathrm{C} \mathrm{NMR}\left(\mathrm{CDCl}_{3}, 100 \mathrm{MHz}, \mathrm{TMS}\right) \delta 20.9$, 
26.6, 55.1, 64.9, 66.4, 104.6, 111.7, 112.1, 114.9, 115.5, 118.8, 127.6, 129.4, 132.0, 159.6, 171.0. IR $(\mathrm{EtOH}) \vee 2939,1732,1611,1576,1509,1486,1453,1364,1225,1154,1127,1103,1034,1002$, 967, 815, $757 \mathrm{~cm}^{-1}$. MS (ESI) m/z $289\left(\mathrm{M}^{+}, 50\right), 198$ (100), 229 (39), 199 (20), 216 (16), 184 (14), 115 (11), 43 (10); HRMS (ESI) calcd. for $\mathrm{C}_{16} \mathrm{H}_{19} \mathrm{NO}_{4}$ : 289.1314, Found: 289.1317.
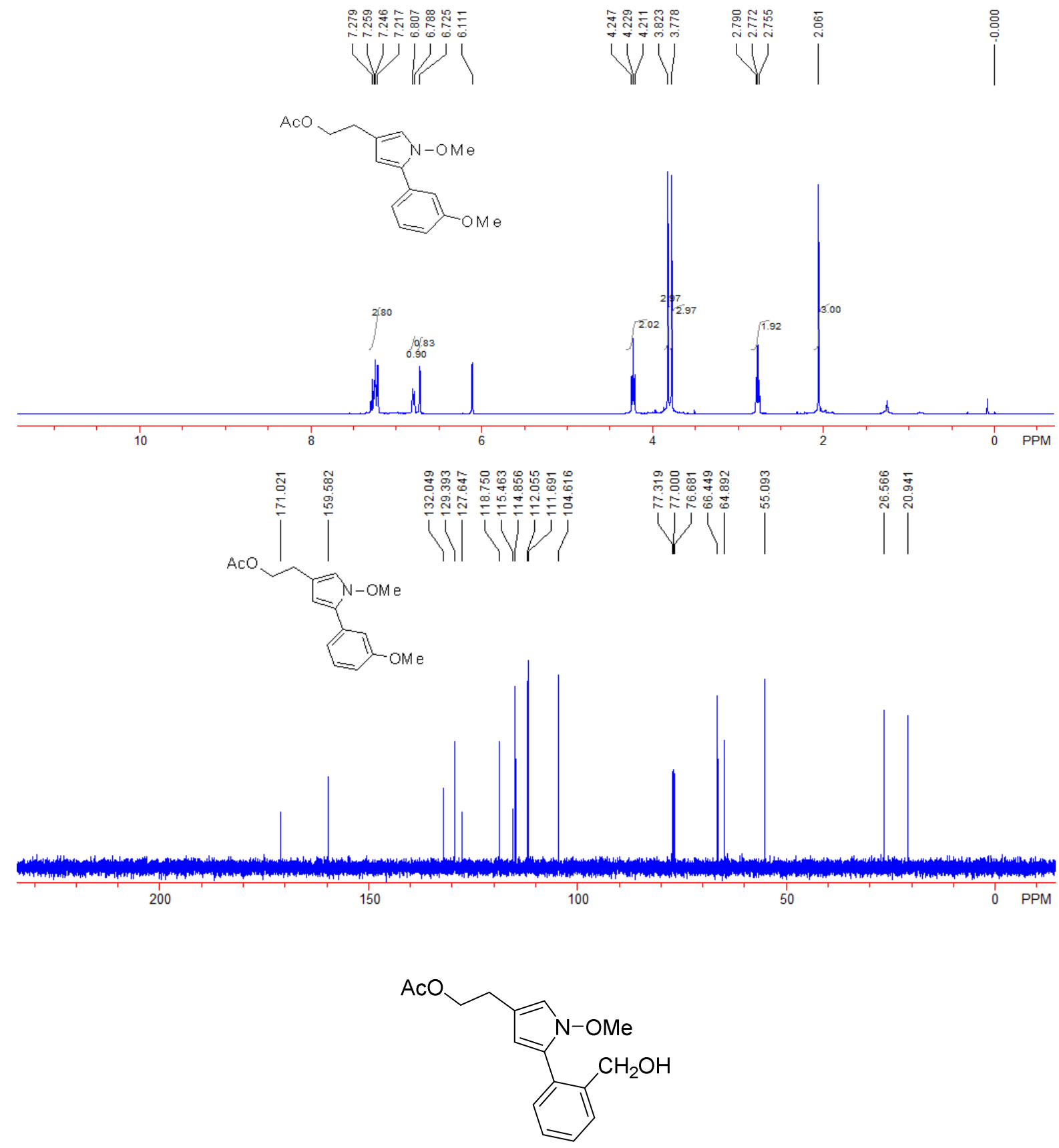

2-(5-(2-(hydroxymethyl)phenyl)-1-methoxy-1H-pyrrol-3-yl)ethyl acetate 2f: Yield: $52.3 \mathrm{mg}$, 91\%; A colorless oil; ${ }^{1} \mathrm{H}$ NMR $\left(\mathrm{CDCl}_{3}, 400 \mathrm{MHz}, \mathrm{TMS}\right) \delta 2.08\left(\mathrm{~s}, 3 \mathrm{H}, \mathrm{CH}_{3}\right), 2.18(\mathrm{t}, J=5.2 \mathrm{~Hz}, 1 \mathrm{H}$, $\mathrm{OH}), 2.80\left(\mathrm{t}, J=7.2 \mathrm{~Hz}, 2 \mathrm{H}, \mathrm{CH}_{2}\right), 3.64\left(\mathrm{~s}, 3 \mathrm{H}, \mathrm{CH}_{3}\right), 4.25\left(\mathrm{t}, J=7.2 \mathrm{~Hz}, 2 \mathrm{H}, \mathrm{CH}_{2}\right), 4.61(\mathrm{~d}, J=5.2$ 
$\mathrm{Hz}, 2 \mathrm{H}, \mathrm{CH}_{2}$ ), 5.94 (d, $\left.J=2.0 \mathrm{~Hz}, 1 \mathrm{H}, \mathrm{Ar}\right), 6.75$ (d, $\left.J=2.0 \mathrm{~Hz}, 1 \mathrm{H}, \mathrm{Ar}\right), 7.26$ (s, 1H, Ar), 7.34-7.42 (m, 3H, Ar), 7.54-7.57 (m, 1H, Ar). ${ }^{13} \mathrm{C}$ NMR ( $\left.\mathrm{CDCl}_{3}, 100 \mathrm{MHz}, \mathrm{TMS}\right) \delta$ 21.1, 26.7, 63.7, 65.0, 67.0, 106.0, 113.6, 115.4, 126.9, 127.5, 128.4, 128.9, 129.5, 130.8, 140.0, 171.2. IR (EtOH) v 3423, 2953, 2922, 2851, 1734, 1439, 1385, 1364, 1240, 1106, 1032, 1000, 969, 802, 765, $668 \mathrm{~cm}^{-1}$. MS (ESI) $m / z 290(\mathrm{M}+\mathrm{H})^{+}$. HRMS (ESI) calcd. for $\mathrm{C}_{16} \mathrm{H}_{20} \mathrm{NO}_{4}$ : 290.1387, Found: 290.1388.

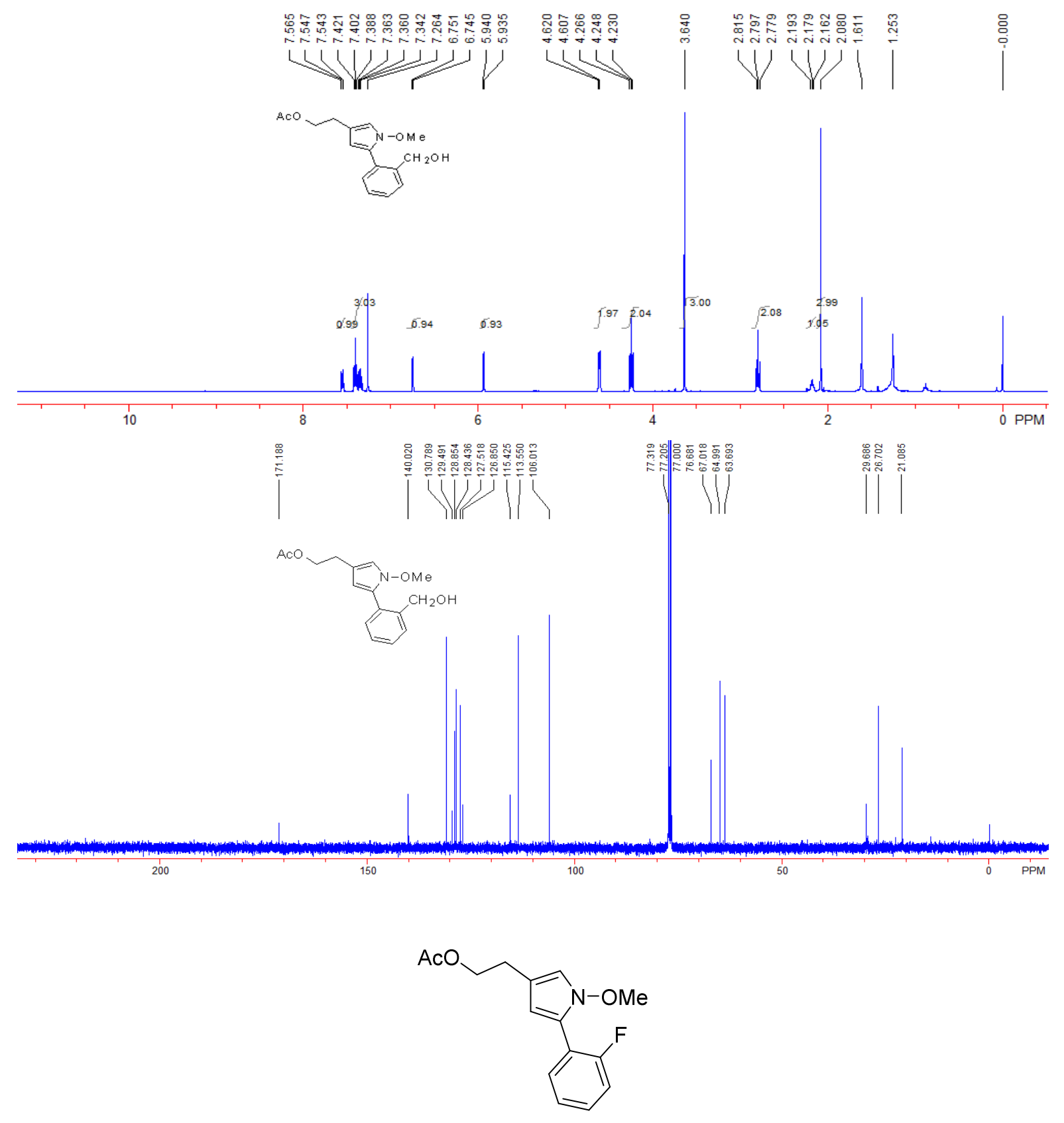

2-(1-acetoxy-5-(2-fluorophenyl)-1H-pyrrol-3-yl)ethyl acetate 2g: Yield: $53.1 \mathrm{mg}, 91 \%$; A red oil; ${ }^{1} \mathrm{H}$ NMR ( $\left.\mathrm{CDCl}_{3}, 400 \mathrm{MHz}, \mathrm{TMS}\right) \delta 1.97\left(\mathrm{~s}, 3 \mathrm{H}, \mathrm{CH}_{3}\right), 2.71\left(\mathrm{t}, J=7.2 \mathrm{~Hz}, 2 \mathrm{H}, \mathrm{CH}_{2}\right), 3.67$ (s, 3H, $\left.\mathrm{CH}_{3}\right), 4.15\left(\mathrm{t}, J=7.2 \mathrm{~Hz}, 2 \mathrm{H}, \mathrm{CH}_{2}\right), 6.11(\mathrm{~d}, J=2.0 \mathrm{~Hz}, 1 \mathrm{H}, \mathrm{Ar}), 6.69$ (d, $\left.J=2.0 \mathrm{~Hz}, 1 \mathrm{H}, \mathrm{Ar}\right)$, 
7.02-7.16 (m, 3H, Ar), 7.56-7.60 (m, 1H, Ar). ${ }^{13} \mathrm{C} \mathrm{NMR}\left(\mathrm{CDCl}_{3}, 100 \mathrm{MHz}, \mathrm{TMS}\right) \delta 20.9,26.6$, 64.9, 66.5, $107.3(\mathrm{~d}, J=6.1 \mathrm{~Hz}), 114.6,115.6(\mathrm{~d}, J=10.5 \mathrm{~Hz}), 115.9,118.7(\mathrm{~d}, J=12.9 \mathrm{~Hz}), 121.4$ $(\mathrm{d}, J=1.5 \mathrm{~Hz}), 123.9(\mathrm{~d}, J=3.8 \mathrm{~Hz}), 128.2(\mathrm{~d}, J=8.3 \mathrm{~Hz}), 129.5(\mathrm{~d}, J=3.0 \mathrm{~Hz}), 159.1(\mathrm{~d}, J=$ 247.4 Hz), 171.0. ${ }^{19} \mathrm{~F}$ NMR (376 MHz, $\left.\mathrm{CDCl}_{3}, \mathrm{CFCl}_{3}\right) \delta$-110.34-(-110.40) (m). IR (EtOH) v 3005, 2939, 2834, 1733, 1600, 1582, 1484, 1463, 1434, 1364, 1236, 1165, 1035, 969, 868, 779, $693 \mathrm{~cm}^{-1}$. MS (EI) m/z 277 (M+, 32), 186 (100), 217 (64), 146 (23), 204 (19), 187 (18), 174 (17), 185 (16). HRMS (EI) calcd. for $\mathrm{C}_{15} \mathrm{H}_{16} \mathrm{FNO}_{3}$ : 277.1114, Found: 277.1125.

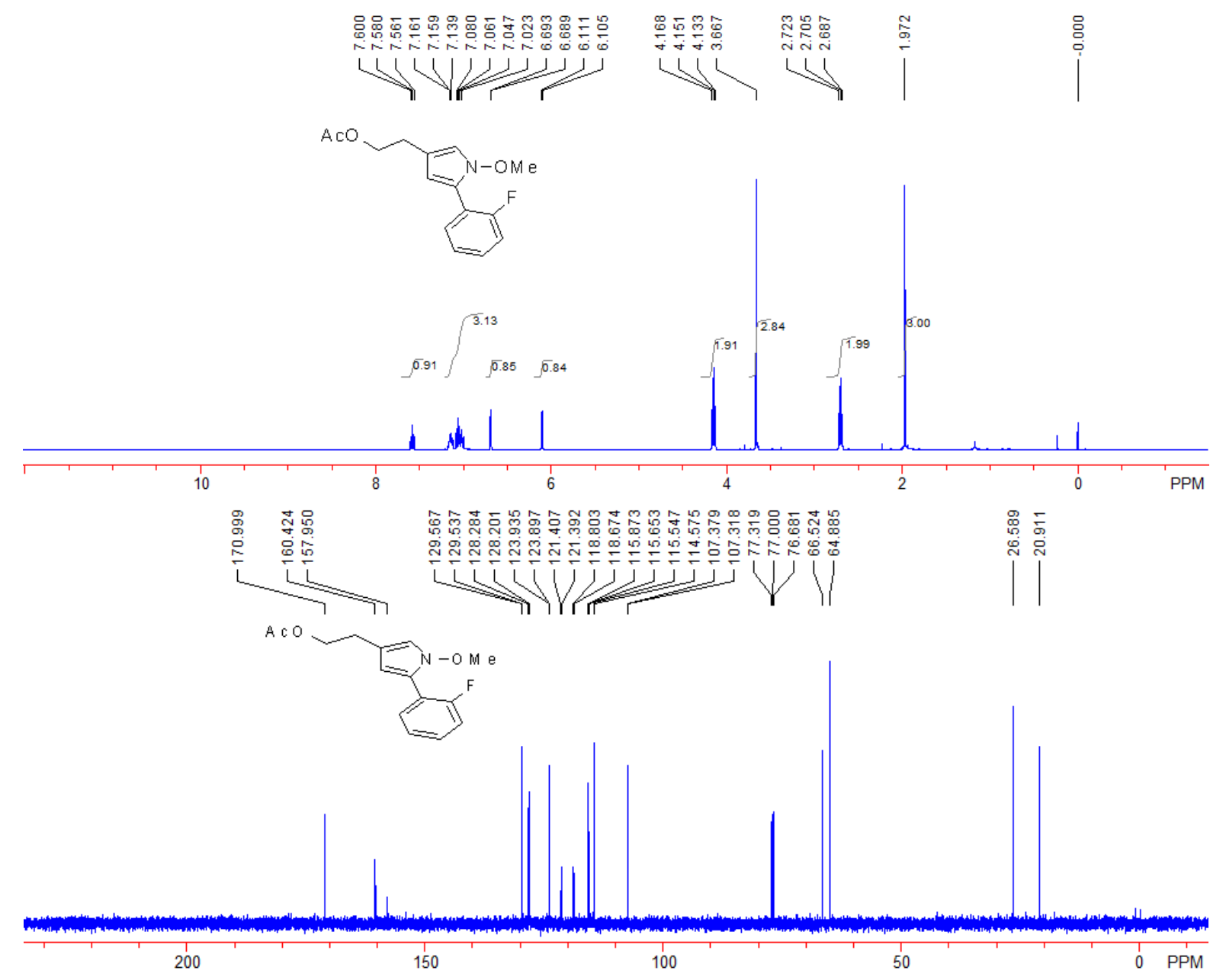




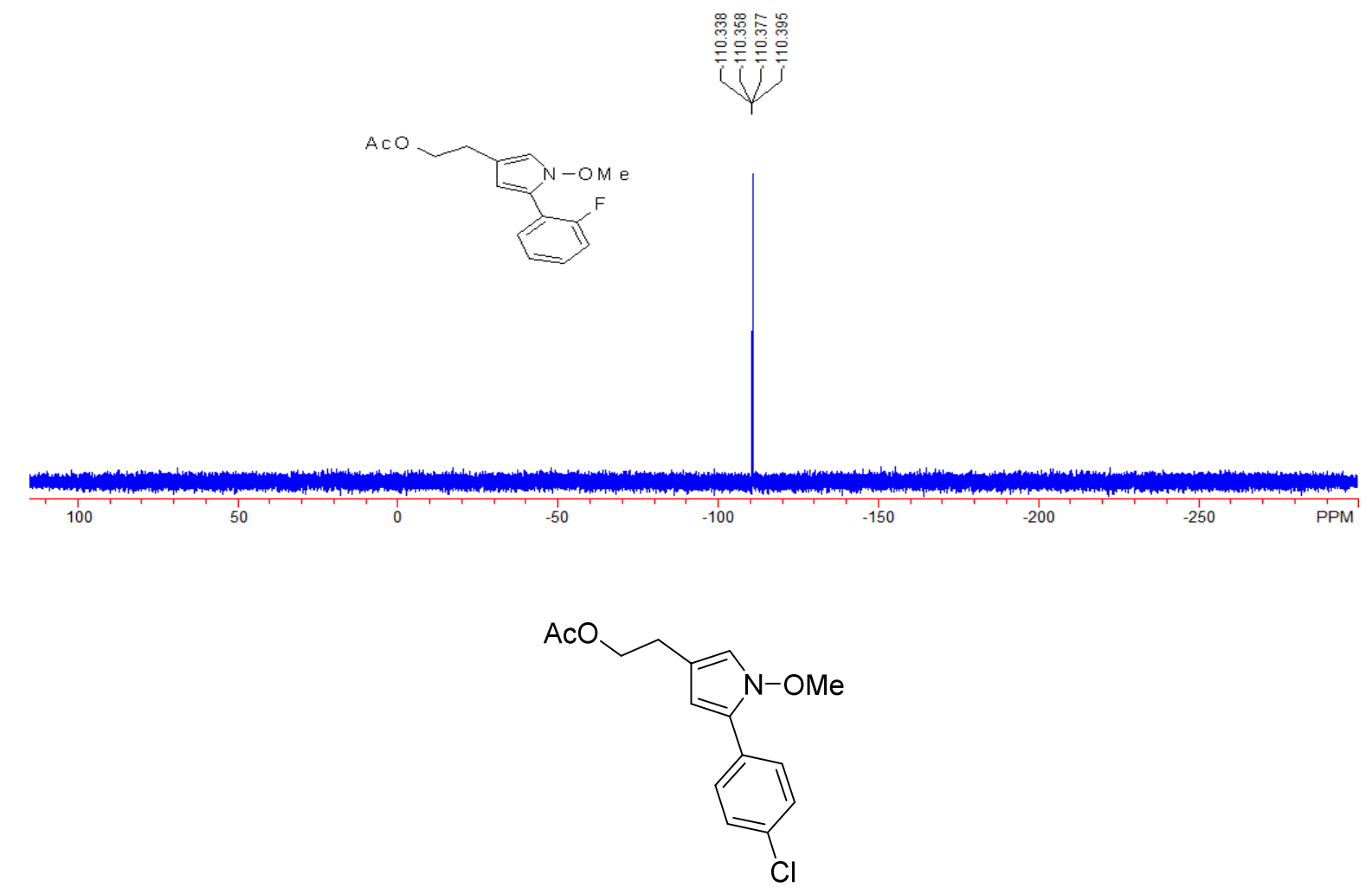

2-(5-(4-chlorophenyl)-1-methoxy-1H-pyrrol-3-yl)ethyl acetate $2 \mathbf{h}$ : Yield: $55.3 \mathrm{mg}, 94 \%$; A colorless oil; ${ }^{1} \mathrm{H} \mathrm{NMR}\left(\mathrm{CDCl}_{3}, 400 \mathrm{MHz}, \mathrm{TMS}\right) \delta 2.08\left(\mathrm{~s}, 3 \mathrm{H}, \mathrm{CH}_{3}\right), 2.78\left(\mathrm{t}, J=7.2 \mathrm{~Hz}, 2 \mathrm{H}, \mathrm{CH}_{2}\right)$, $3.77\left(\mathrm{~s}, 3 \mathrm{H}, \mathrm{CH}_{3}\right), 4.23$ (t, $\left.J=7.2 \mathrm{~Hz}, 2 \mathrm{H}, \mathrm{CH}_{2}\right), 6.10$ (d, $\left.J=2.4 \mathrm{~Hz}, 1 \mathrm{H}, \mathrm{Ar}\right), 6.74$ (d, $J=2.4 \mathrm{~Hz}$, 1H, Ar), 7.33-7.35 (m, 2H, Ar), 7.57-7.59 (m, 2H, Ar). ${ }^{13} \mathrm{C} \mathrm{NMR}\left(\mathrm{CDCl}_{3}, 100 \mathrm{MHz}, \mathrm{TMS}\right) \delta 21.1$, 26.6, 64.9, 66.6, 104.7, 115.1, 115.7, 126.8, 127.4, 128.7, 129.3, 132.2, 171.1. IR (EtOH) v 2922, $2850,1732,1698,1594,1490,1438,1364,1235,1091,1035,1013,831,728 \mathrm{~cm}^{-1}$. MS (ESI) $\mathrm{m} / z$ $294(\mathrm{M}+\mathrm{H})^{+}$. HRMS (ESI) calcd. for $\mathrm{C}_{15} \mathrm{H}_{17} \mathrm{ClNO}_{3}$ : 294.0891, Found: 294.0891.

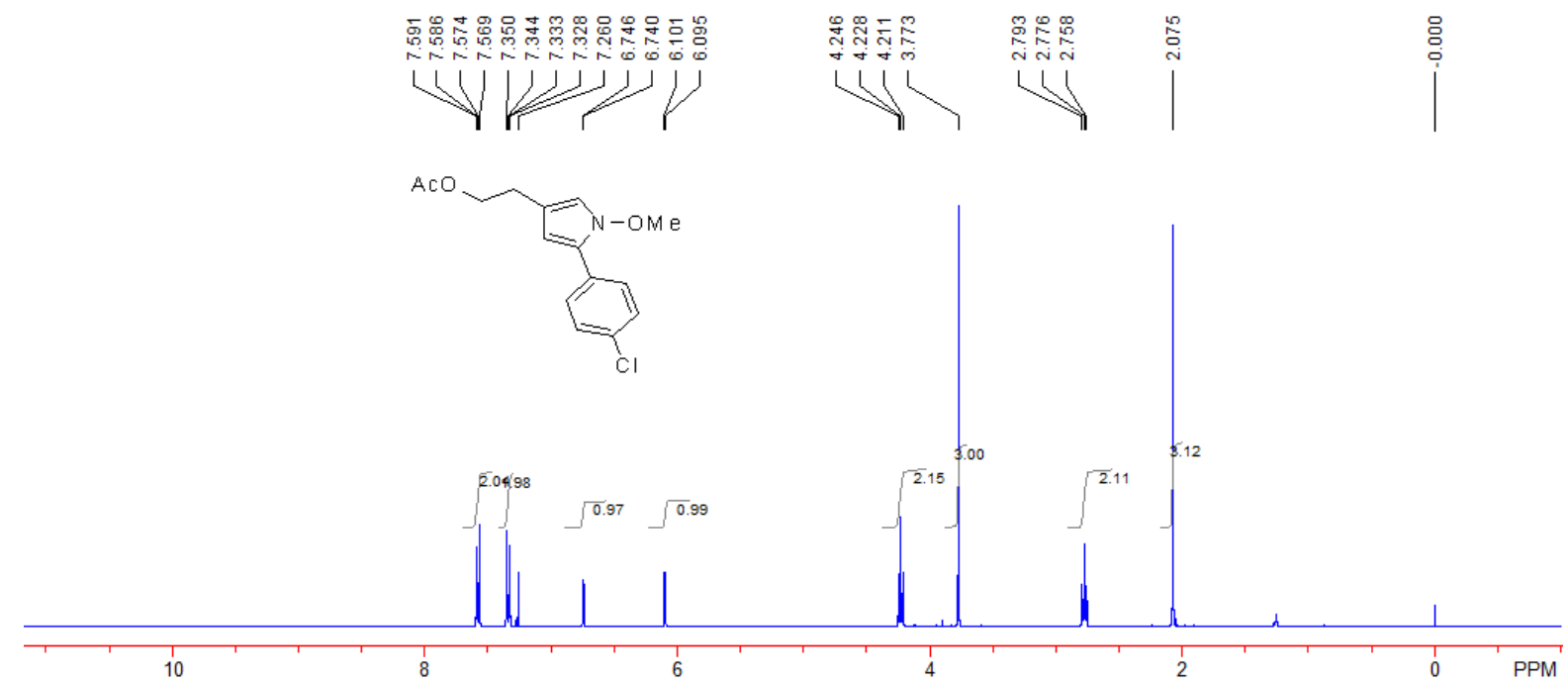




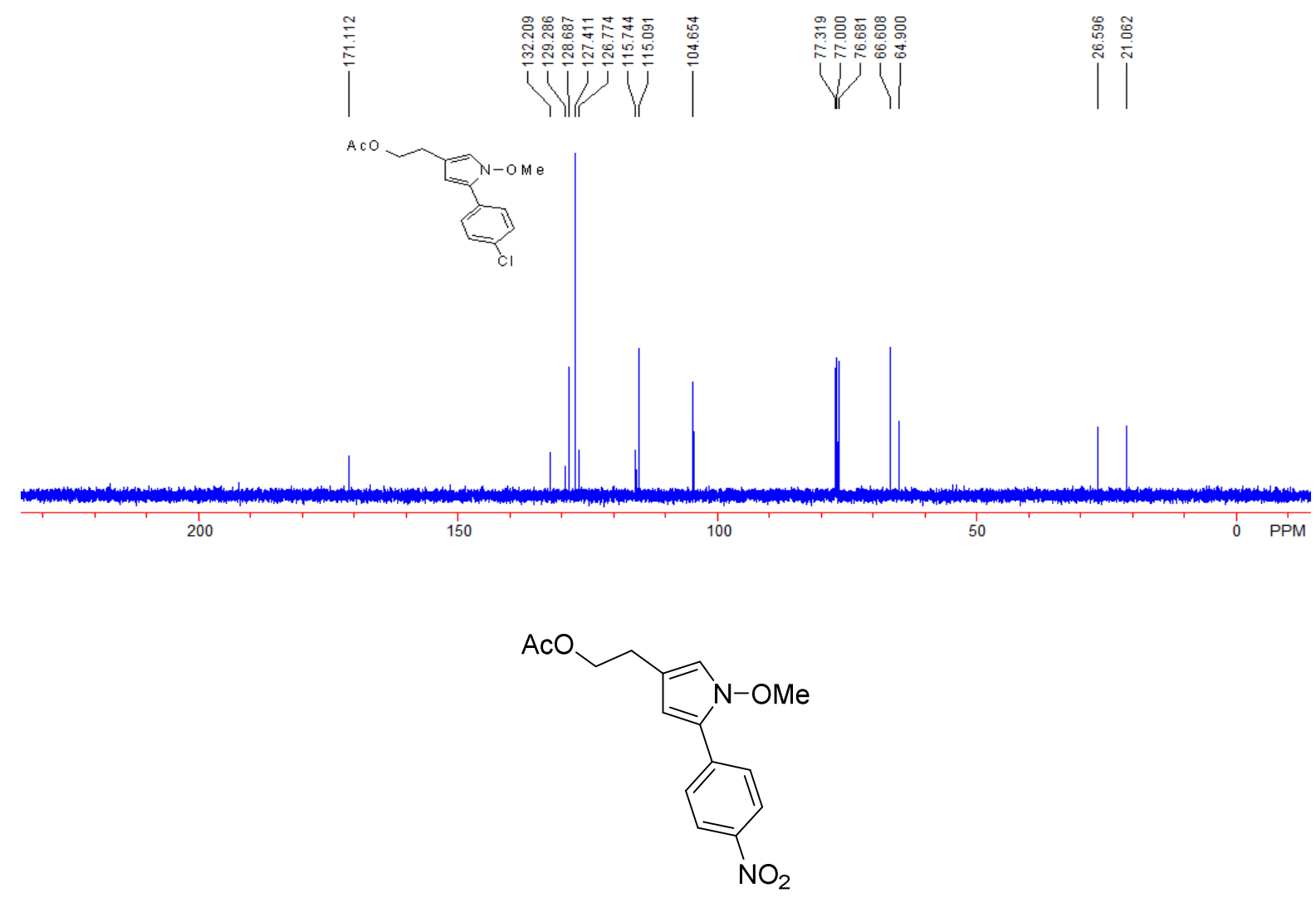

2-(1-methoxy-5-(4-nitrophenyl)-1H-pyrrol-3-yl)ethyl acetate 2i: Yield: $56.9 \mathrm{mg}, 93 \%$; A colorless oil; ${ }^{1} \mathrm{H}$ NMR $\left(\mathrm{CDCl}_{3}, 400 \mathrm{MHz}, \mathrm{TMS}\right) \delta 2.08\left(\mathrm{~s}, 3 \mathrm{H}, \mathrm{CH}_{3}\right), 2.80$ (t, $\left.J=7.2 \mathrm{~Hz}, 2 \mathrm{H}, \mathrm{CH}_{2}\right), 3.84$ (s, $\left.3 \mathrm{H}, \mathrm{CH}_{3}\right), 4.24$ (t, $\left.J=7.2 \mathrm{~Hz}, 2 \mathrm{H}, \mathrm{CH}_{2}\right), 6.31$ (d, $\left.J=2.0 \mathrm{~Hz}, 1 \mathrm{H}, \mathrm{Ar}\right), 6.86$ (d, $\left.J=2.0 \mathrm{~Hz}, 1 \mathrm{H}, \mathrm{Ar}\right)$, $7.81(\mathrm{~d}, J=9.2 \mathrm{~Hz}, 2 \mathrm{H}, \mathrm{Ar}), 8.22(\mathrm{~d}, J=9.2 \mathrm{~Hz}, 2 \mathrm{H}, \mathrm{Ar}) .{ }^{13} \mathrm{C} \mathrm{NMR}\left(\mathrm{CDCl}_{3}, 100 \mathrm{MHz}, \mathrm{TMS}\right) \delta$ 21.0, 26.5, 64.7, 67.0, 107.2, 116.8, 117.4, 124.1, 125.5, 125.7, 136.9, 145.5, 171.0. IR (EtOH) $v$ 3113, 2956, 2935, 2853, 1732, 1593, 1512, 1437, 1384, 1332, 1237, 1109, 1032, 1000, 963, 852, 802, 752, $693 \mathrm{~cm}^{-1}$. MS (ESI) $\mathrm{m} / z 305(\mathrm{M}+\mathrm{H})^{+}$. HRMS (ESI) calcd. for $\mathrm{C}_{15} \mathrm{H}_{17} \mathrm{~N}_{2} \mathrm{O}_{5}$ : 305.1132 , Found: 305.1132. 


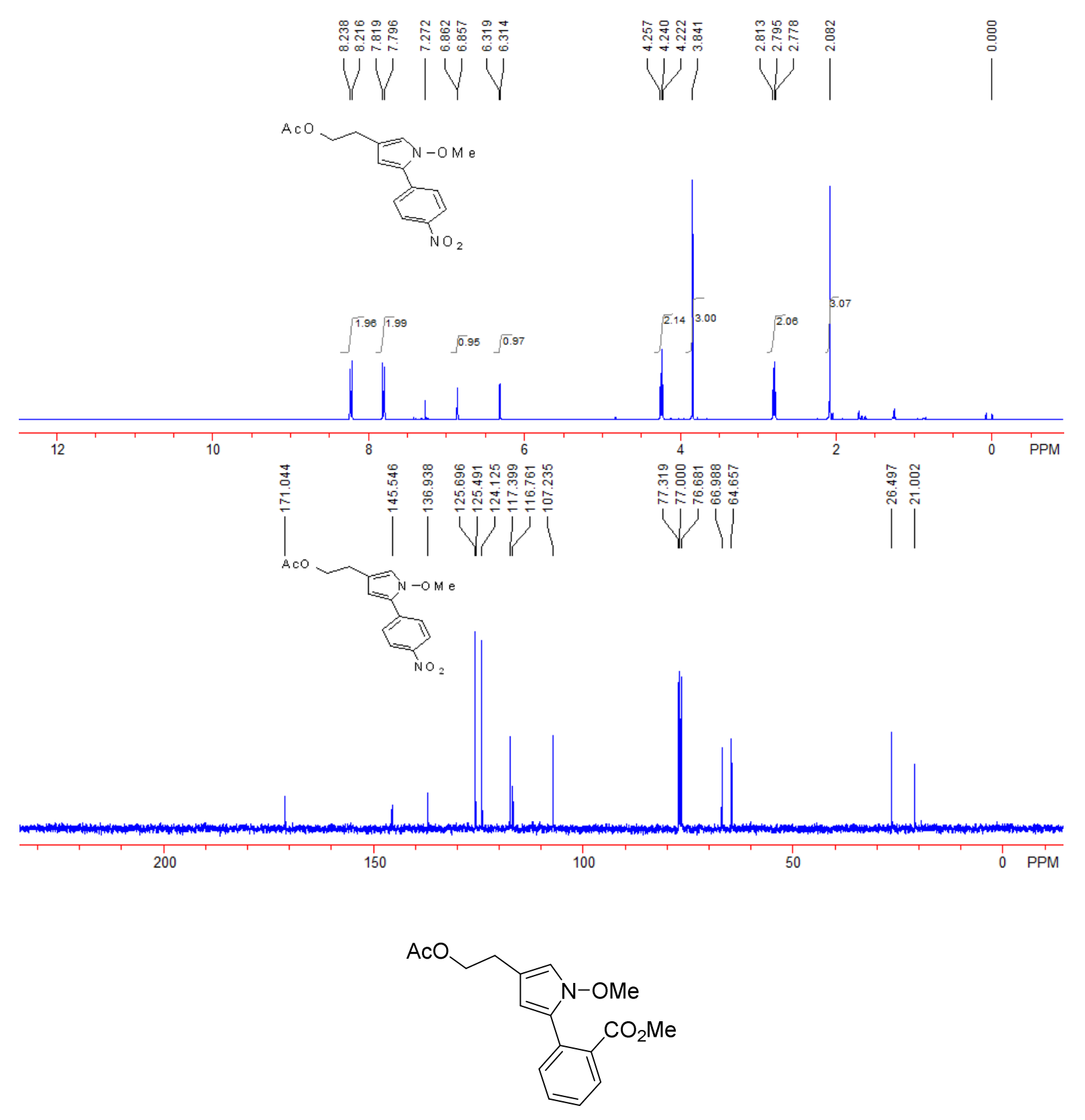

methyl 2-(4-(2-acetoxyethyl)-1-methoxy-1H-pyrrol-2-yl)benzoate 2j: Yield: $56.8 \mathrm{mg}, 89 \%$; A red oil; ${ }^{1} \mathrm{H}$ NMR $\left(\mathrm{CDCl}_{3}, 400 \mathrm{MHz}, \mathrm{TMS}\right) \delta 2.08\left(\mathrm{~s}, 3 \mathrm{H}, \mathrm{CH}_{3}\right), 2.80\left(\mathrm{t}, J=7.2 \mathrm{~Hz}, 2 \mathrm{H}, \mathrm{CH}_{2}\right), 3.60(\mathrm{~s}$, $\left.3 \mathrm{H}, \mathrm{CH}_{3}\right), 3.76\left(\mathrm{~s}, 3 \mathrm{H}, \mathrm{CH}_{3}\right), 4.25$ (t, $\left.J=7.2 \mathrm{~Hz}, 2 \mathrm{H}, \mathrm{CH}_{2}\right), 6.00$ (s, 1H, Ar), 6.73 (s, 1H, Ar), 7.38-7.40 (m, 1H, Ar), 7.46-7.52 (m, 2H, Ar), 7.81-7.83 (m, 1H, Ar). ${ }^{13} \mathrm{C} \mathrm{NMR}\left(\mathrm{CDCl}_{3}, 100 \mathrm{MHz}\right.$, TMS) $\delta 21.0,26.7,52.2,65.0,66.7,104.7,113.8,115.2,127.1,129.8,130.1,130.7,130.8,131.3$, 169.0, 171.1. IR (EtOH) v 3064, 2950, 2899, 1786, 1720, 1647, 1600, 1433, 1365, 1291, 1236, 1122, 1083, 1042, 996, 930, 796, 762, 714, $691 \mathrm{~cm}^{-1}$. MS (ESI) m/z $318(\mathrm{M}+\mathrm{H})^{+}$. HRMS (ESI) calcd. for $\mathrm{C}_{17} \mathrm{H}_{20} \mathrm{NO}_{5}$ : 318.1336, Found: 318.1337 . 


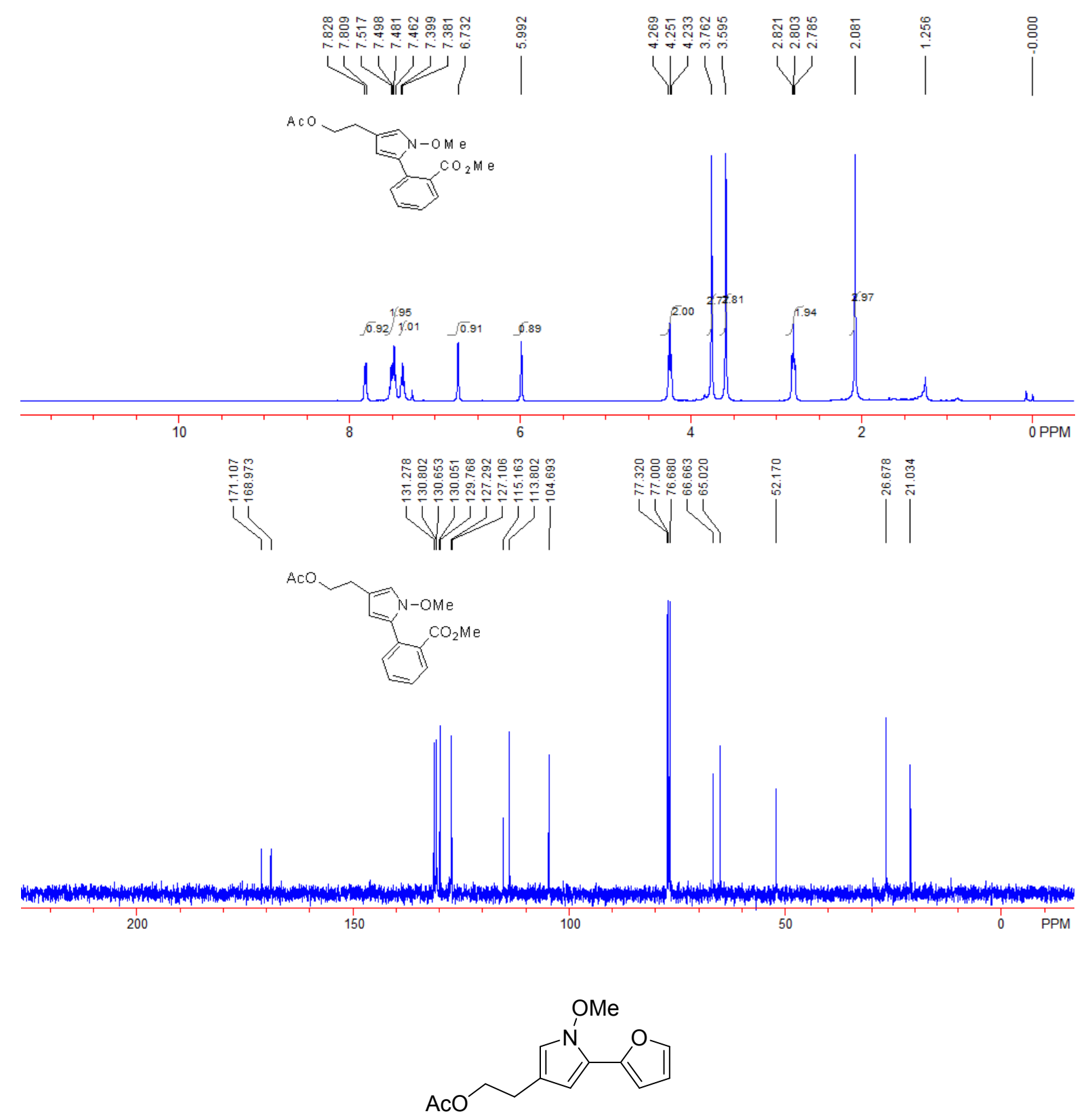

2-(1-acetoxy-5-(2-fluorophenyl)-1H-pyrrol-3-yl)ethyl acetate 2k: Yield: $52.9 \mathrm{mg}, 95 \%$; A colorless oil; ${ }^{1} \mathrm{H}$ NMR $\left(\mathrm{CDCl}_{3}, 400 \mathrm{MHz}, \mathrm{TMS}\right) \delta 2.07$ (s, 3H, $\mathrm{CH}_{3}$ ), 2.77 (t, J=7.2 Hz, 2H, $\mathrm{CH}_{2}$ ), 3.95 (s, $\left.3 \mathrm{H}, \mathrm{CH}_{3}\right), 4.22\left(\mathrm{t}, J=7.2 \mathrm{~Hz}, 2 \mathrm{H}, \mathrm{CH}_{2}\right), 6.19$ (d, $\left.J=2.0 \mathrm{~Hz}, 1 \mathrm{H}, \mathrm{Ar}\right), 6.44-6.46$ (m, 1H, Ar), 6.51 (d, $J=3.2 \mathrm{~Hz}, 1 \mathrm{H}, \mathrm{Ar}), 6.69(\mathrm{~d}, J=2.0 \mathrm{~Hz}, 1 \mathrm{H}, \mathrm{Ar}), 7.39$ (s, $1 \mathrm{H}, \mathrm{Ar}) .{ }^{13} \mathrm{C} \mathrm{NMR}\left(\mathrm{CDCl}_{3}, 100 \mathrm{MHz}\right.$, TMS) $\delta 21.1,26.6,29.7,64.9,66.4,103.0,104.7,111.2,114.2,115.8,120.3,140.7,145.6,171.1$. IR $(\mathrm{EtOH}) \vee 2955,2919,2851,1737,1569,1457,1398,1360,1246,1098,1022 \mathrm{~cm}^{-1}$. MS (ESI) $m / z 250(\mathrm{M}+\mathrm{H})^{+}$. HRMS (ESI) calcd. for $\mathrm{C}_{13} \mathrm{H}_{16} \mathrm{NO}_{4}$ : 250.1074, Found: 250.1078 . 


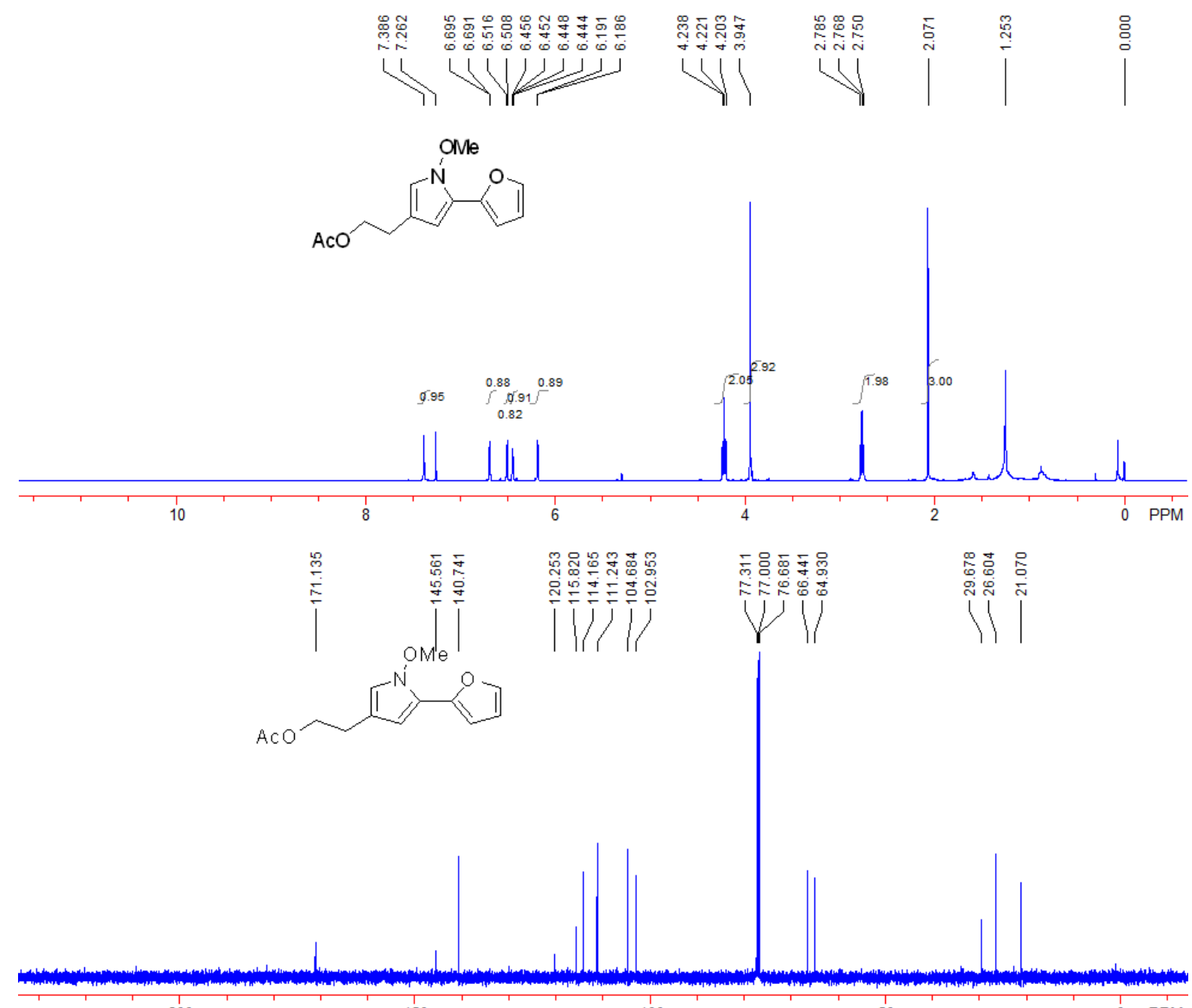

PPM

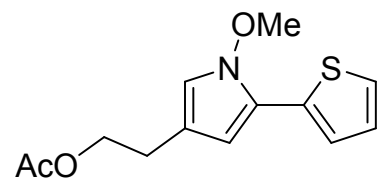

2-(1-methoxy-5-(thiophen-2-yl)-1H-pyrrol-3-yl)ethyl acetate 21: Yield: $47.9 \mathrm{mg}, 90 \%$; A colorless oil; ${ }^{1} \mathrm{H}$ NMR $\left(\mathrm{CDCl}_{3}, 400 \mathrm{MHz}, \mathrm{TMS}\right) \delta 2.07$ (s, 3H, $\mathrm{CH}_{3}$ ), 2.76 (t, $J=7.2 \mathrm{~Hz}, 2 \mathrm{H}, \mathrm{CH}_{2}$ ), 3.92 (s, $\left.3 \mathrm{H}, \mathrm{CH}_{3}\right), 4.22\left(\mathrm{t}, J=7.2 \mathrm{~Hz}, 2 \mathrm{H}, \mathrm{CH}_{2}\right), 6.12(\mathrm{~d}, J=2.0 \mathrm{~Hz}, 1 \mathrm{H}, \mathrm{Ar}), 6.71(\mathrm{~d}, J=2.0 \mathrm{~Hz}, 1 \mathrm{H}, \mathrm{Ar})$, 7.03-7.04 (m, 1H, Ar) , 7.19-7.21 (m, 1H, Ar) , 7.25-7.26 (m, 1H, Ar). ${ }^{13} \mathrm{C} \mathrm{NMR}\left(\mathrm{CDCl}_{3}, 100 \mathrm{MHz}\right.$, TMS) $\delta 21.1,26.6,64.9,66.6,103.5,114.0,115.9,122.77,122.83,123.5,127.2,132.3,171.1$. IR $(\mathrm{EtOH}) \vee 3101,2926,2853,1734,1575,1525,1438,1381,1363,1238,1123,1032,973,940,846$, $789,694 \mathrm{~cm}^{-1}$. MS (ESI) $\mathrm{m} / z 266(\mathrm{M}+\mathrm{H})^{+}$. HRMS (ESI) calcd. for $\mathrm{C}_{13} \mathrm{H}_{16} \mathrm{NO}_{3} \mathrm{~S}: 266.0845$, Found: 266.0848 . 


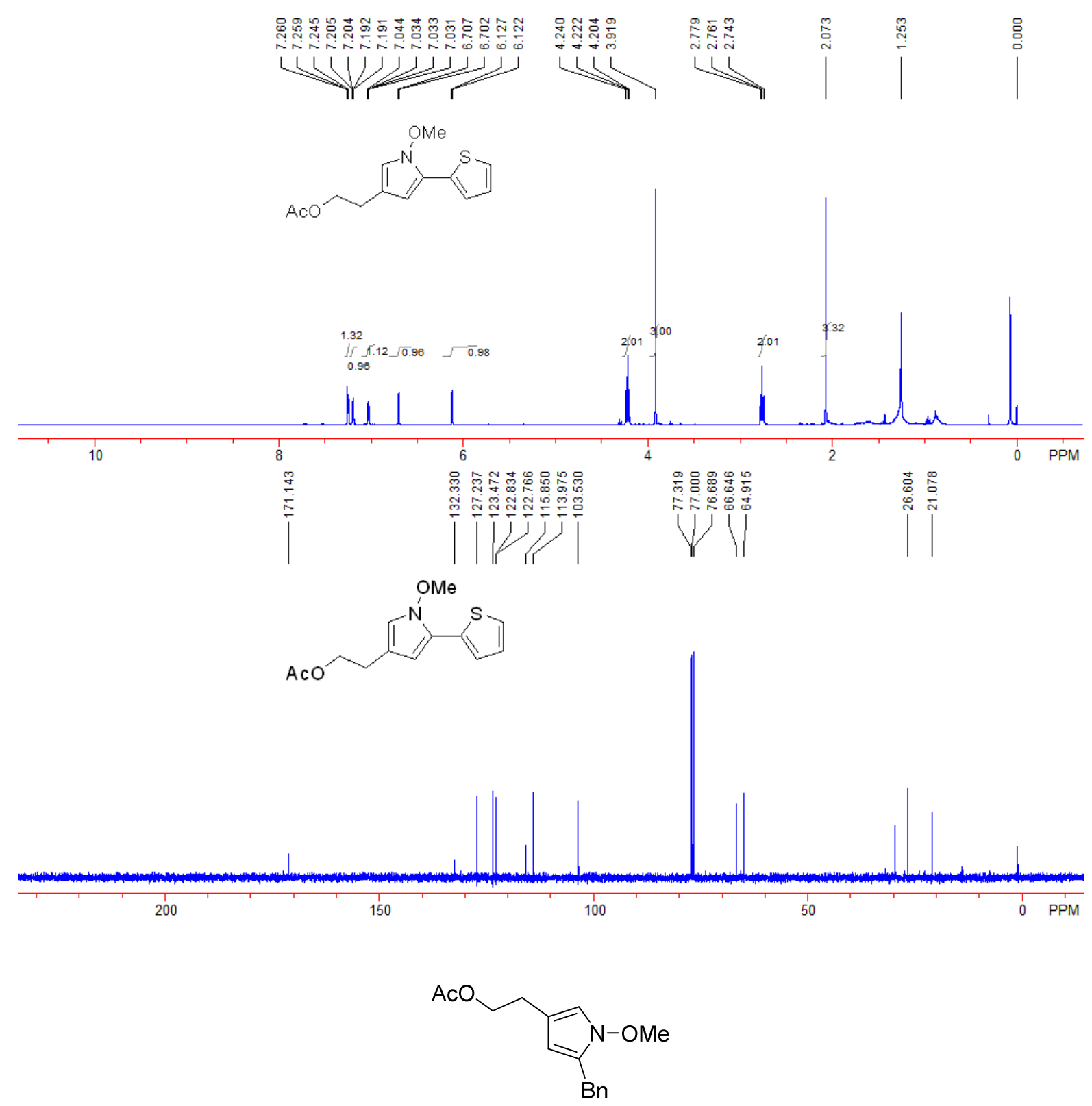

2-(5-benzyl-1-methoxy-1H-pyrrol-3-yl)ethyl acetate 2m: Yield: 41.6mg, 76\%; A colorless oil; ${ }^{1} \mathrm{H}$ NMR $\left(\mathrm{CDCl}_{3}, 400 \mathrm{MHz}, \mathrm{TMS}\right) \delta 2.05\left(\mathrm{~s}, 3 \mathrm{H}, \mathrm{CH}_{3}\right), 2.71\left(\mathrm{t}, J=7.2 \mathrm{~Hz}, 2 \mathrm{H}, \mathrm{CH}_{2}\right), 3.75(\mathrm{~s}, 3 \mathrm{H}$, $\left.\mathrm{CH}_{3}\right), 3.91\left(\mathrm{~s}, 2 \mathrm{H}, \mathrm{CH}_{2}\right), 4.18$ (t, $\left.J=7.2 \mathrm{~Hz}, 2 \mathrm{H}, \mathrm{CH}_{2}\right), 5.57$ (d, $\left.J=1.2 \mathrm{~Hz}, 1 \mathrm{H}, \mathrm{Ar}\right), 6.59$ (d, $J=1.2$ $\mathrm{Hz}, 1 \mathrm{H}, \mathrm{Ar}), 7.20-7.29$ (m, 5H, Ar). ${ }^{13} \mathrm{C} \mathrm{NMR}\left(\mathrm{CDCl}_{3}, 100 \mathrm{MHz}, \mathrm{TMS}\right) \delta$ 21.1, 26.7, 31.2, 65.1, $66.6,103.2,111.8,114.5,126.2,127.5,128.4,128.6,139.2$, 171.2. IR (EtOH) v 2923, 2852, 1736, 1494, 1454, 1363, 1239, 1031, 975, 732, $699 \mathrm{~cm}^{-1}$. MS (ESI) $\mathrm{m} / z 274(\mathrm{M}+\mathrm{H})^{+}$. HRMS (ESI) calcd. for $\mathrm{C}_{16} \mathrm{H}_{20} \mathrm{NO}_{3}$ : 274.1438, Found: 274.1440. 

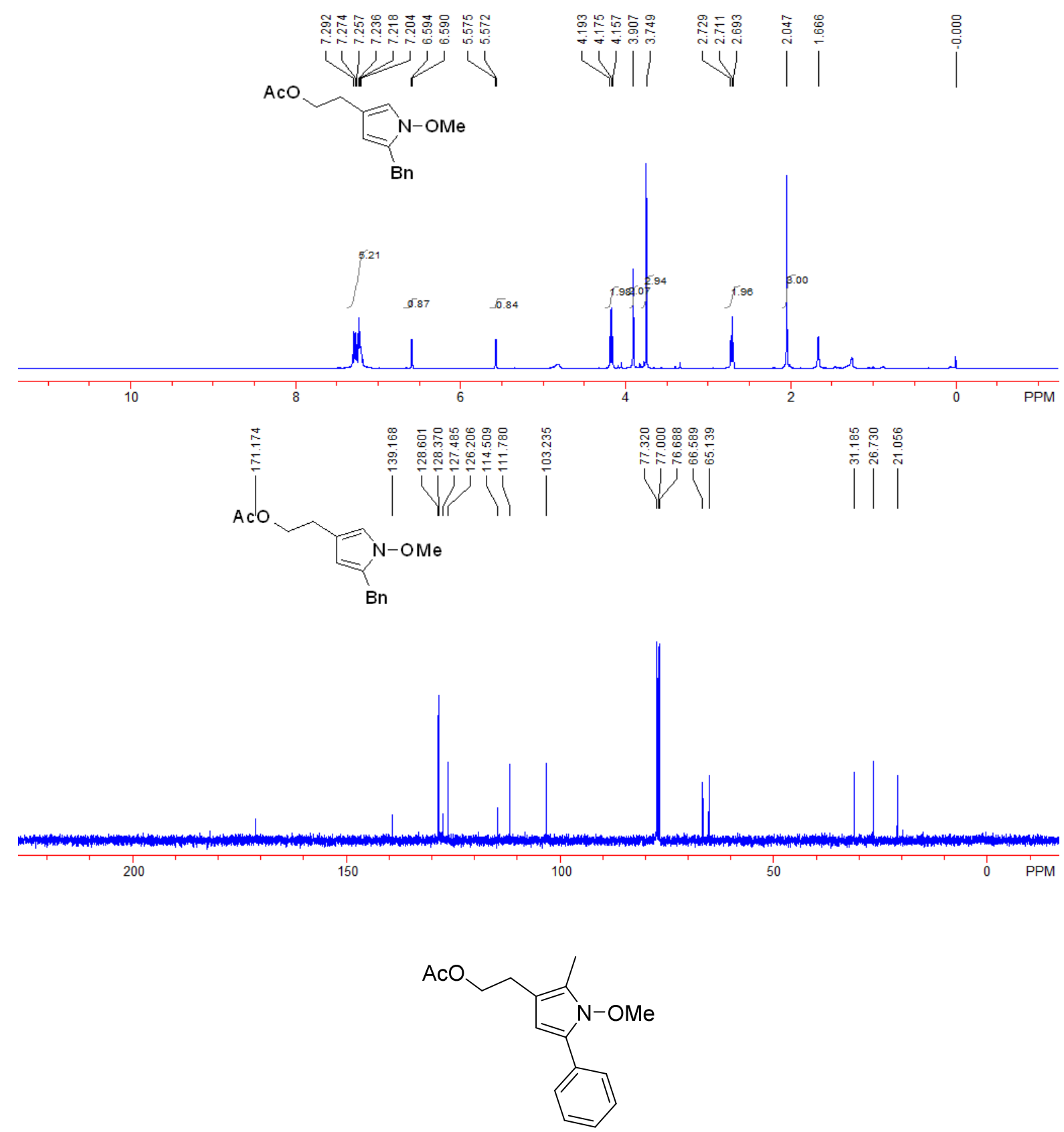

2-(1-methoxy-2-methyl-5-phenyl-1H-pyrrol-3-yl)ethyl acetate 2n: Yield: $52.0 \mathrm{mg}, 96 \%$; A colorless oil; ${ }^{1} \mathrm{H}$ NMR $\left(\mathrm{CDCl}_{3}, 400 \mathrm{MHz}, \mathrm{TMS}\right) \delta 2.06\left(\mathrm{~s}, 3 \mathrm{H}, \mathrm{CH}_{3}\right), 2.26\left(\mathrm{~s}, 3 \mathrm{H}, \mathrm{CH}_{3}\right), 2.73$ (t, $J=7.2 \mathrm{~Hz}$, 2H, $\left.\mathrm{CH}_{2}\right), 3.68\left(\mathrm{~s}, 3 \mathrm{H}, \mathrm{CH}_{3}\right), 4.18\left(\mathrm{t}, J=7.2 \mathrm{~Hz}, 2 \mathrm{H}, \mathrm{CH}_{2}\right), 6.08(\mathrm{~s}, 1 \mathrm{H}, \mathrm{Ar}), 7.19-7.25(\mathrm{~m}, 1 \mathrm{H}, \mathrm{Ar})$, 7.33-7.37 (m, 2H, Ar), 7.64-7.66 (m, 2H, Ar). ${ }^{13} \mathrm{C} \mathrm{NMR}\left(\mathrm{CDCl}_{3}, 100 \mathrm{MHz}, \mathrm{TMS}\right) \delta$ 8.4, 21.0, 25.8, 65.0, 65.2, 103.8, 111.5, 123.3, 125.9, 126.0, 126.1, 128.5, 131.3, 171.1. IR (EtOH) v 3059, 2934, $2855,2827,1735,1636,1603,1548,1515,1447,1384,1363,1239,1131,1029,971,907,797,756$, 720, $695 \mathrm{~cm}^{-1}$. MS (ESI) $\mathrm{m} / z 274(\mathrm{M}+\mathrm{H})^{+}$. HRMS (ESI) calcd. for $\mathrm{C}_{16} \mathrm{H}_{20} \mathrm{NO}_{3}$ : 274.1438, Found: 274.1441 . 


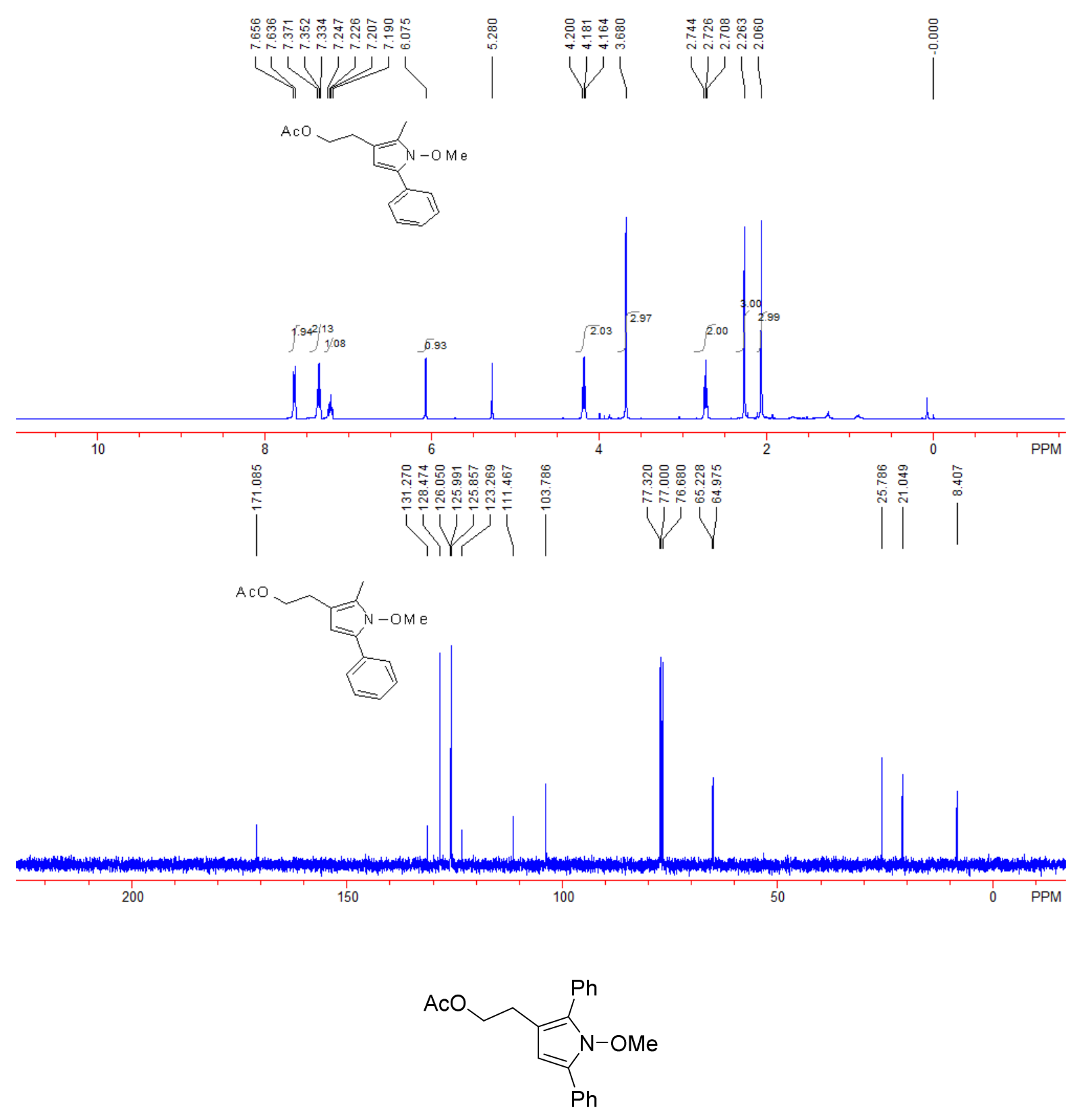

2-(1-methoxy-2,5-diphenyl-1H-pyrrol-3-yl)ethyl acetate 2o: Yield: 53.9 mg, 80\%; A colorless oil; ${ }^{1} \mathrm{H}$ NMR $\left(\mathrm{CDCl}_{3}, 400 \mathrm{MHz}, \mathrm{TMS}\right) \delta 2.00\left(\mathrm{~s}, 3 \mathrm{H}, \mathrm{CH}_{3}\right), 2.88\left(\mathrm{t}, J=7.2 \mathrm{~Hz}, 2 \mathrm{H}, \mathrm{CH}_{2}\right), 3.37$ (s, 3H, $\mathrm{CH}_{3}$ ), 4.23 (t, $\left.J=7.2 \mathrm{~Hz}, 2 \mathrm{H}, \mathrm{CH}_{2}\right), 6.25$ (s, 1H, Ar), 7.24-7.55 (m, 6H, Ar), 7.56 (d, J=7.2 Hz, 2H, Ar), 7.73 (d, $J=7.2 \mathrm{~Hz}, 2 \mathrm{H}, \mathrm{Ar}) .{ }^{13} \mathrm{C} \mathrm{NMR}\left(\mathrm{CDCl}_{3}, 100 \mathrm{MHz}, \mathrm{TMS}\right) \delta$ 21.0, 25.8, 64.9, 65.1, 105.1, $114.4,126.4,126.6,127.1,128.2,128.3,128.5,128.7,129.3,130.1,130.9,171.1$. IR (EtOH) $v$ $3057,2933,2899,2814,1736,1600,1491,1443,1384,1363,1231,1181,1052,1032,962,907$, 883, 850, 799, 754, $692 \mathrm{~cm}^{-1}$. MS (ESI) $\mathrm{m} / z 336(\mathrm{M}+\mathrm{H})^{+}$. HRMS (ESI) calcd. for $\mathrm{C}_{21} \mathrm{H}_{22} \mathrm{NO}_{3}$ : 336.1594, Found: 336.1596. 

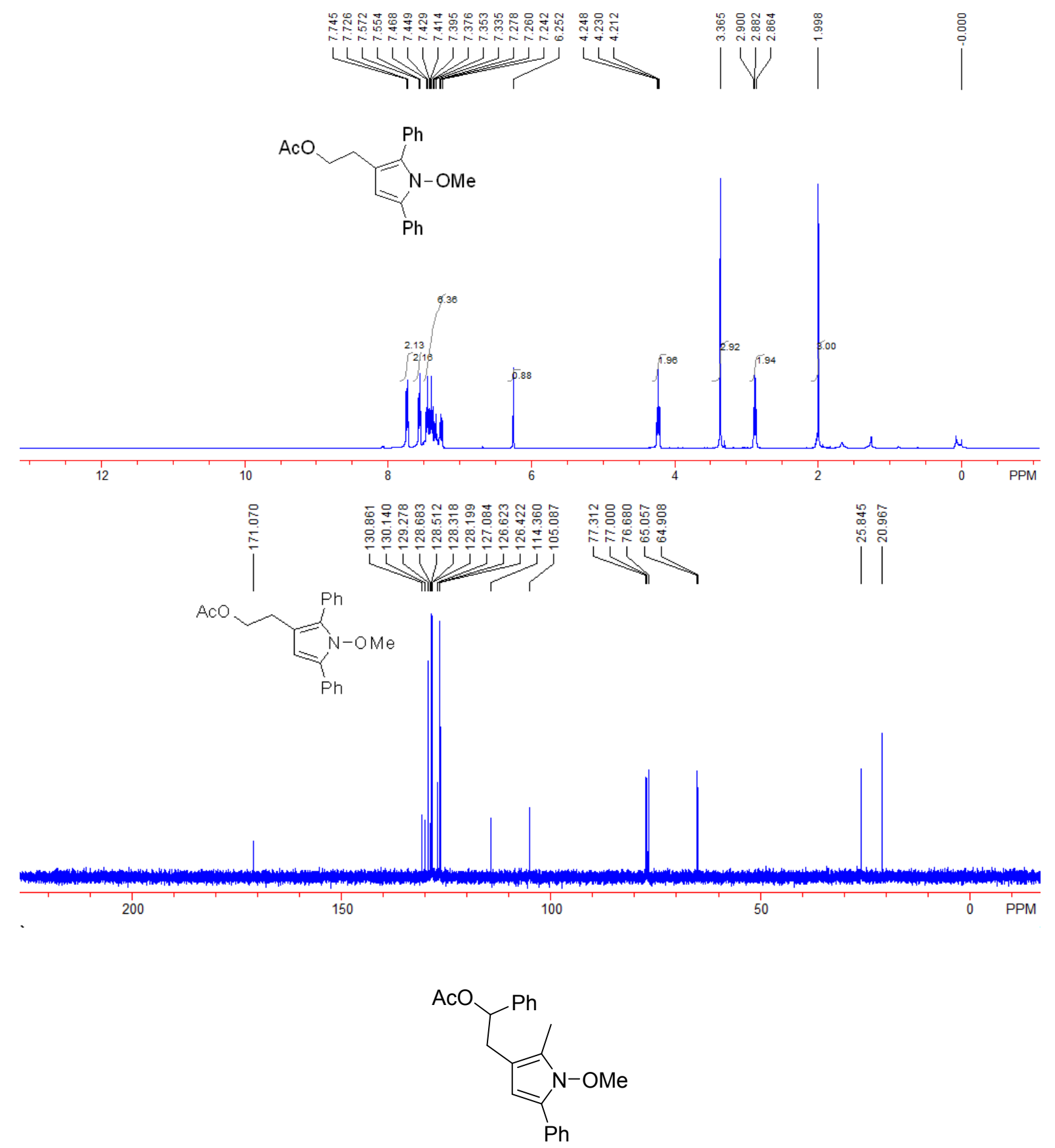

2-(1-methoxy-2-methyl-5-phenyl-1H-pyrrol-3-yl)-1-phenylethyl acetate 2p: Yield: $63.7 \mathrm{mg}, 91 \%$; A colorless oil; ${ }^{1} \mathrm{H}$ NMR $\left(\mathrm{CDCl}_{3}, 400 \mathrm{MHz}, \mathrm{TMS}\right) \delta 1.94\left(\mathrm{~s}, 3 \mathrm{H}, \mathrm{CH}_{3}\right), 2.08\left(\mathrm{~s}, 3 \mathrm{H}, \mathrm{CH}_{3}\right), 2.83(\mathrm{dd}$, $\left.J_{1}=14.4 \mathrm{~Hz}, J_{2}=6.8 \mathrm{~Hz}, 1 \mathrm{H}, \mathrm{CH}_{2}\right), 2.98\left(\mathrm{dd}, J_{1}=14.4 \mathrm{~Hz}, J_{2}=7.2 \mathrm{~Hz}, 1 \mathrm{H}, \mathrm{CH}_{2}\right), 3.61(\mathrm{~s}, 3 \mathrm{H}$, $\left.\mathrm{CH}_{3}\right), 5.81(\mathrm{t}, J=7.2 \mathrm{~Hz}, 1 \mathrm{H}, \mathrm{CH}), 5.99$ (s, 1H, Ar), 7.20-7.37 (m, 8H, Ar), 7.61-7.63 (m, 2H, Ar). ${ }^{13} \mathrm{C} \mathrm{NMR}\left(\mathrm{CDCl}_{3}, 100 \mathrm{MHz}, \mathrm{TMS}\right) \delta 8.1,21.4,33.8,65.2,76.8,104.6,110.8,124.1,125.8,125.9$, 126.0, 126.6, 127.7, 128.1, 128.5, 131.4, 140.4, 170.2. IR (EtOH) v 3064, 3028, 2921, 2852, 1738, $1659,1602,1516,1494,1454,1372,1236,1074,1023,972,796,757,697 \mathrm{~cm}^{-1}$. MS (ESI) $m / z 350$ 
$(\mathrm{M}+\mathrm{H})^{+}$. HRMS (ESI) calcd. for $\mathrm{C}_{22} \mathrm{H}_{24} \mathrm{NO}_{3}$ : 350.1751, Found: 350.1754 .
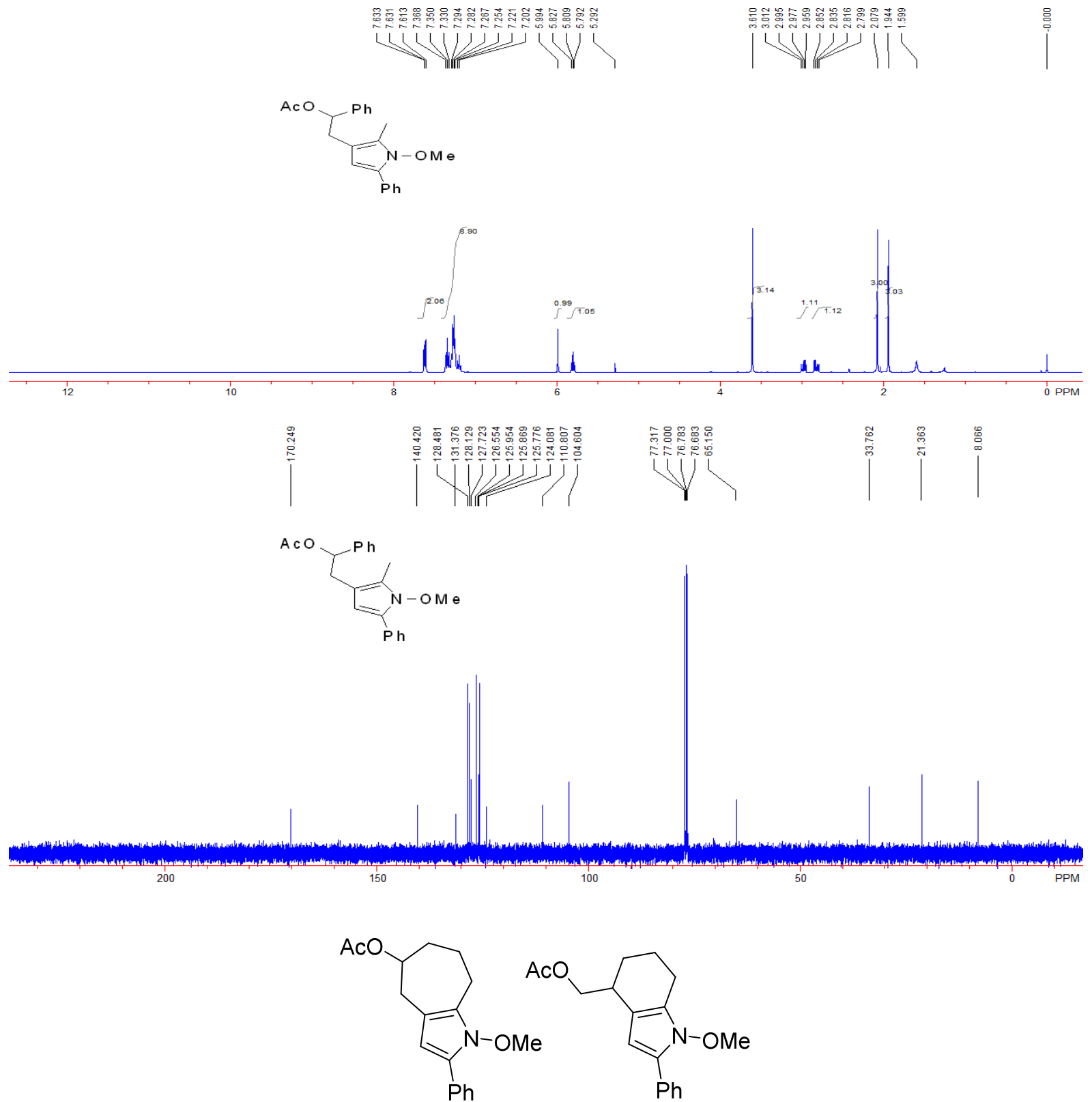

methoxy-2-phenyl-1,4,5,6,7,8-hexahydrocyclohepta[b]pyrrol-5-yl acetate $\quad \mathbf{2 q} \quad$ and (1-methoxy-2-phenyl-4,5,6,7-tetrahydro-1H-indol-4-yl)methyl acetate 2q': The mixture was obtained as a light yellow oil (54.1 mg, 90\% yield), a 4.2/1 mixture of $\mathbf{2 q} / \mathbf{2 q}$ ' which could not be separated by column chromatography. Major isomer 2q: ${ }^{1} \mathrm{H}$ NMR $\left(\mathrm{CDCl}_{3}, 400 \mathrm{MHz}, \mathrm{TMS}\right) \delta$ 1.60-1.69 (m, 1H, $\left.\mathrm{CH}_{2}\right), 1.77-1.86\left(\mathrm{~m}, 1 \mathrm{H}, \mathrm{CH}_{2}\right), 1.92-2.00\left(\mathrm{~m}, 1 \mathrm{H}, \mathrm{CH}_{2}\right), 2.03\left(\mathrm{~s}, 3 \mathrm{H}, \mathrm{CH}_{3}\right)$, 2.17-2.22 (m, $\left.1 \mathrm{H}, \mathrm{CH}_{2}\right), 2.55-2.61\left(\mathrm{~m}, 1 \mathrm{H}, \mathrm{CH}_{2}\right), 2.75-2.87\left(\mathrm{~m}, 2 \mathrm{H}, 2 \mathrm{CH}_{2}\right), 2.92-2.97\left(\mathrm{~m}, 1 \mathrm{H}, \mathrm{CH}_{2}\right)$, $3.62\left(\mathrm{~s}, 3 \mathrm{H}, \mathrm{CH}_{3}\right), 4.83-4.88(\mathrm{~m}, 1 \mathrm{H}, \mathrm{CH}), 6.02(\mathrm{~s}, 1 \mathrm{H}, \mathrm{Ar}), 7.17-7.23(\mathrm{~m}, 1 \mathrm{H}, \mathrm{Ar}), 7.32-7.35(\mathrm{~m}, 2 \mathrm{H}$, $\mathrm{Ar}), 7.62-7.65$ (m, 2H, Ar). ${ }^{13} \mathrm{C} \mathrm{NMR}\left(\mathrm{CDCl}_{3}, 100 \mathrm{MHz}, \mathrm{TMS}\right) \delta$ 21.4, 24.0, 24.1, 34.1, 37.2, 65.6, 
$73.3,104.9,111.2,125.1,125.8,125.9,128.4,128.5,131.2,170.2$.

Minor isomer 2q': ${ }^{1} \mathrm{H}$ NMR $\left(\mathrm{CDCl}_{3}, 400 \mathrm{MHz}, \mathrm{TMS}\right) \delta$ 1.49-1.56 (m, 1H, $\left.\mathrm{CH}_{2}\right), 1.77-1.86(\mathrm{~m}, 2 \mathrm{H}$, $\left.2 \mathrm{CH}_{2}\right), 1.92-2.00\left(\mathrm{~m}, 1 \mathrm{H}, \mathrm{CH}_{2}\right), 2.08\left(\mathrm{~s}, 3 \mathrm{H}, \mathrm{CH}_{3}\right), 2.64-2.66\left(\mathrm{~m}, 2 \mathrm{H}, \mathrm{CH}_{2}\right), 2.92-2.97(\mathrm{~m}, 1 \mathrm{H}, \mathrm{CH})$, $3.69\left(\mathrm{~s}, 3 \mathrm{H}, \mathrm{CH}_{3}\right), 4.06\left(\mathrm{dd}, J_{1}=10.8 \mathrm{~Hz}, J_{2}=8.4 \mathrm{~Hz}, 1 \mathrm{H}, \mathrm{CH}_{2}\right), 4.25\left(\mathrm{dd}, J_{1}=10.8 \mathrm{~Hz}, J_{2}=6.0 \mathrm{~Hz}\right.$, $\left.1 \mathrm{H}, \mathrm{CH}_{2}\right), 6.12$ (s, 1H, Ar), 7.17-7.23 (m, 1H, Ar), 7.32-7.35 (m, 2H, Ar), 7.62-7.65 (m, 2H, Ar). ${ }^{13} \mathrm{C}$ NMR $\left(\mathrm{CDCl}_{3}, 100 \mathrm{MHz}, \mathrm{TMS}\right) \delta$ 20.4, 20.6, 21.0, 26.5, 32.9, 65.3, 68.2, 101.7, 113.4, 125.9, 126.0, $126.1,126.5,128.4,131.3,171.1$.

IR $(\mathrm{EtOH}) \vee 3080,3051,2933,2850,2814,1737,1621,1596,1492,1462,1441,1242,1137,1047$, 1000, 926, 913, 883, 863, 755, 731, 691, $668 \mathrm{~cm}^{-1}$. MS (ESI) $\mathrm{m} / z 300(\mathrm{M}+\mathrm{H})^{+}$. HRMS (ESI) calcd. for $\mathrm{C}_{18} \mathrm{H}_{22} \mathrm{NO}_{3}: 300.1594$, Found: 300.1596 .

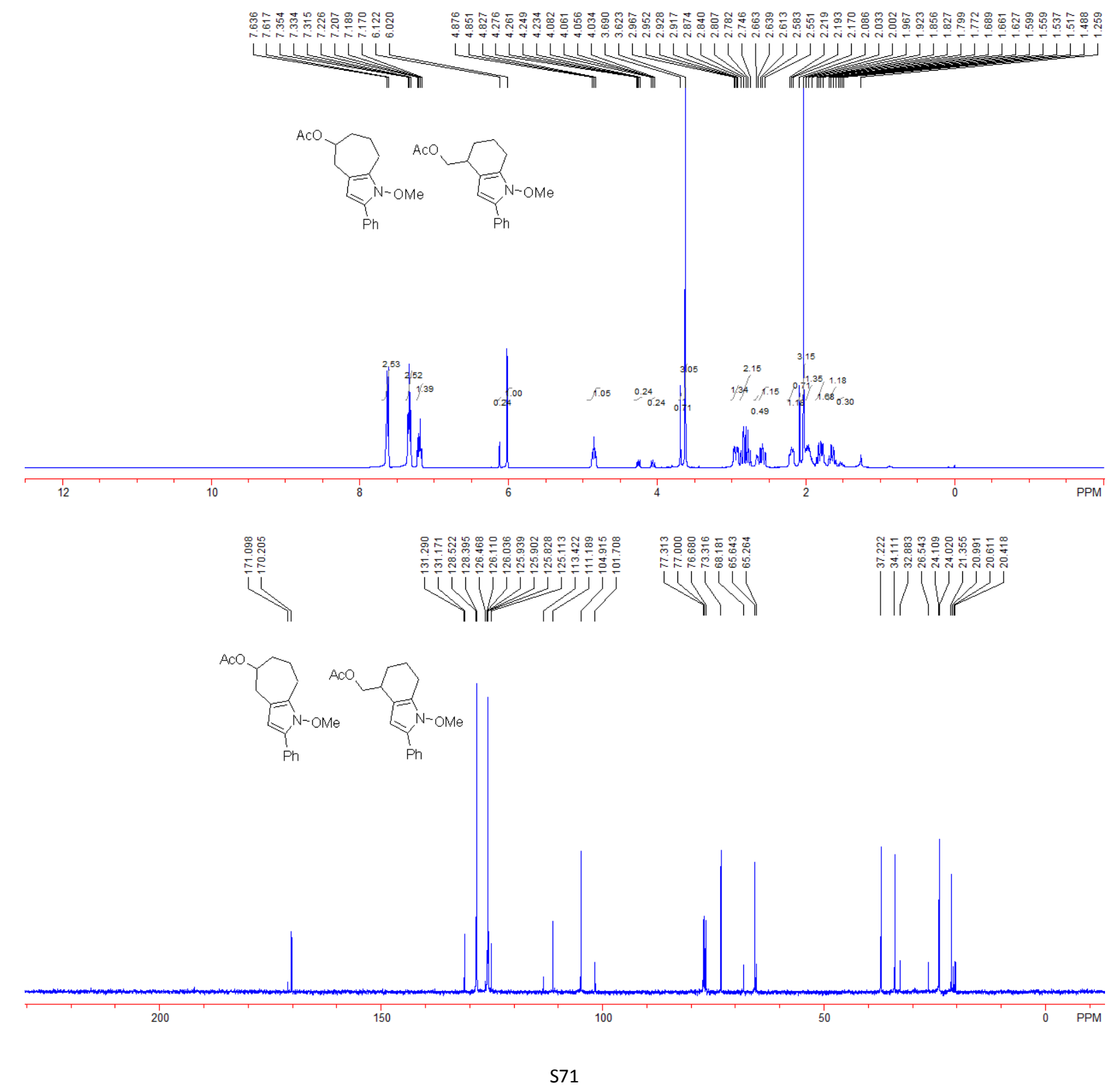




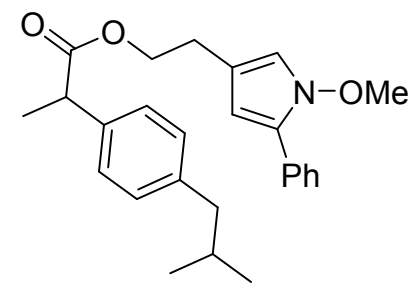

2-(1-methoxy-5-phenyl-1H-pyrrol-3-yl)ethyl 2-(4-isobutylphenyl)propanoate 3a: Yield: $133 \mathrm{mg}$, 82\%; A colorless oil; ${ }^{1} \mathrm{H}$ NMR $\left(\mathrm{CDCl}_{3}, 400 \mathrm{MHz}, \mathrm{TMS}\right) \delta 0.89\left(\mathrm{~d}, J=6.4 \mathrm{~Hz}, 6 \mathrm{H}, 2 \mathrm{CH}_{3}\right), 1.50$ (d, $\left.J=7.6 \mathrm{~Hz}, 3 \mathrm{H}, \mathrm{CH}_{3}\right), 1.79-1.86(\mathrm{~m}, 1 \mathrm{H}, \mathrm{CH}), 2.43\left(\mathrm{~d}, J=7.2 \mathrm{~Hz}, 2 \mathrm{H}, \mathrm{CH}_{2}\right), 2.72(\mathrm{t}, J=6.4 \mathrm{~Hz}, 2 \mathrm{H}$, $\left.\mathrm{CH}_{2}\right), 3.68-3.73(\mathrm{~m}, 1 \mathrm{H}, \mathrm{CH}), 3.72\left(\mathrm{~s}, 3 \mathrm{H}, \mathrm{CH}_{3}\right), 4.20-4.26\left(\mathrm{~m}, 2 \mathrm{H}, \mathrm{CH}_{2}\right), 6.02(\mathrm{~d}, J=2.4 \mathrm{~Hz}, 1 \mathrm{H}$, Ar), 6.58 (d, $J=2.4 \mathrm{~Hz}, 1 \mathrm{H}, \mathrm{Ar}), 7.08$ (d, $J=7.6 \mathrm{~Hz}, 2 \mathrm{H}, \mathrm{Ar}), 7.20-7.26$ (m, 3H, Ar), 7.34-7.38 (m, 2H, Ar), 7.62 (d, $J=7.6 \mathrm{~Hz}, 2 \mathrm{H}, \mathrm{Ar}) .{ }^{13} \mathrm{C} \mathrm{NMR}\left(\mathrm{CDCl}_{3}, 100 \mathrm{MHz}, \mathrm{TMS}\right) \delta 18.5,22.4,26.6,30.1$, $45.0,45.2,65.3,66.4,104.5,114.8,115.6,126.3,126.5,127.2,127.8,128.5,129.3,130.9,137.8$, 140.4, 174.7. IR $(\mathrm{EtOH}) \vee 2954,2867,1730,1602,1509,1449,1381,1331,1240,1200,1162$, 1124, 1092, 1071, 1021, 1005, 994, 966, 848, 799, 758, 694, $665 \mathrm{~cm}^{-1}$. MS (ESI) $\mathrm{m} / z$ $406(\mathrm{M}+\mathrm{H})^{+}$. HRMS (ESI) calcd. for $\mathrm{C}_{26} \mathrm{H}_{32} \mathrm{NO}_{3}$ : 406.2377, Found: 406.2379.
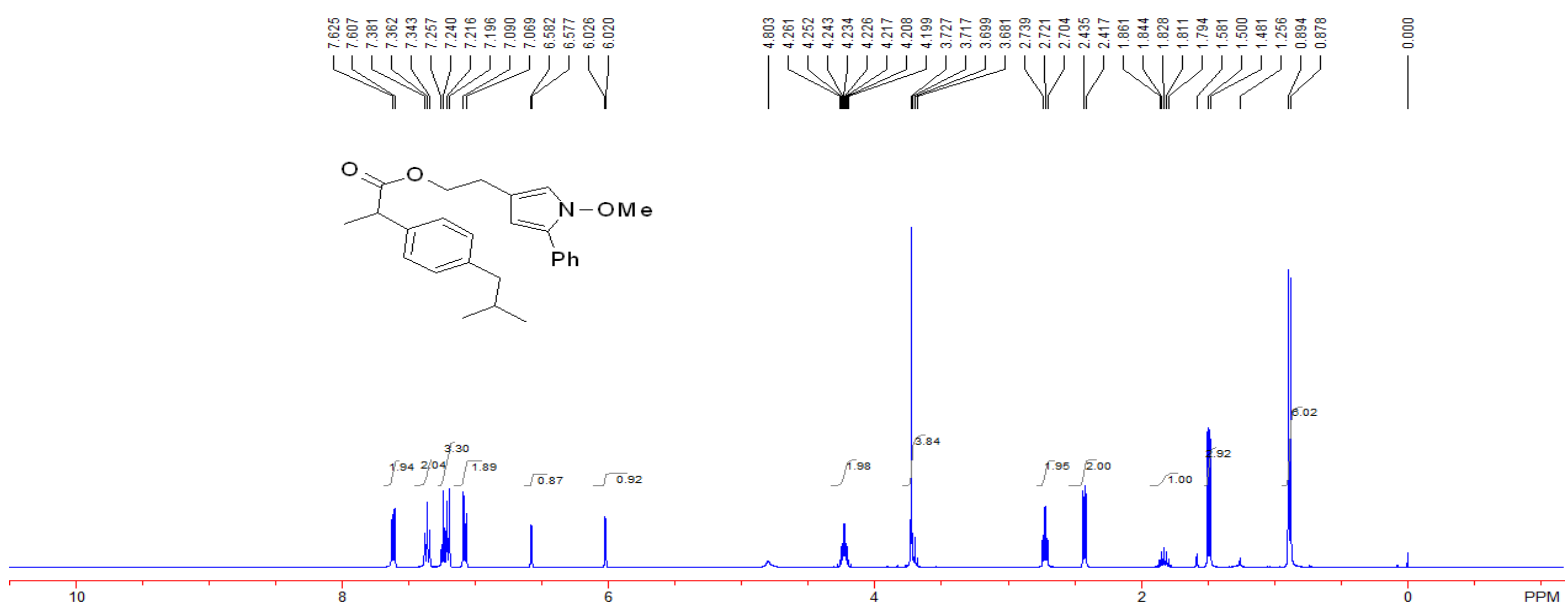

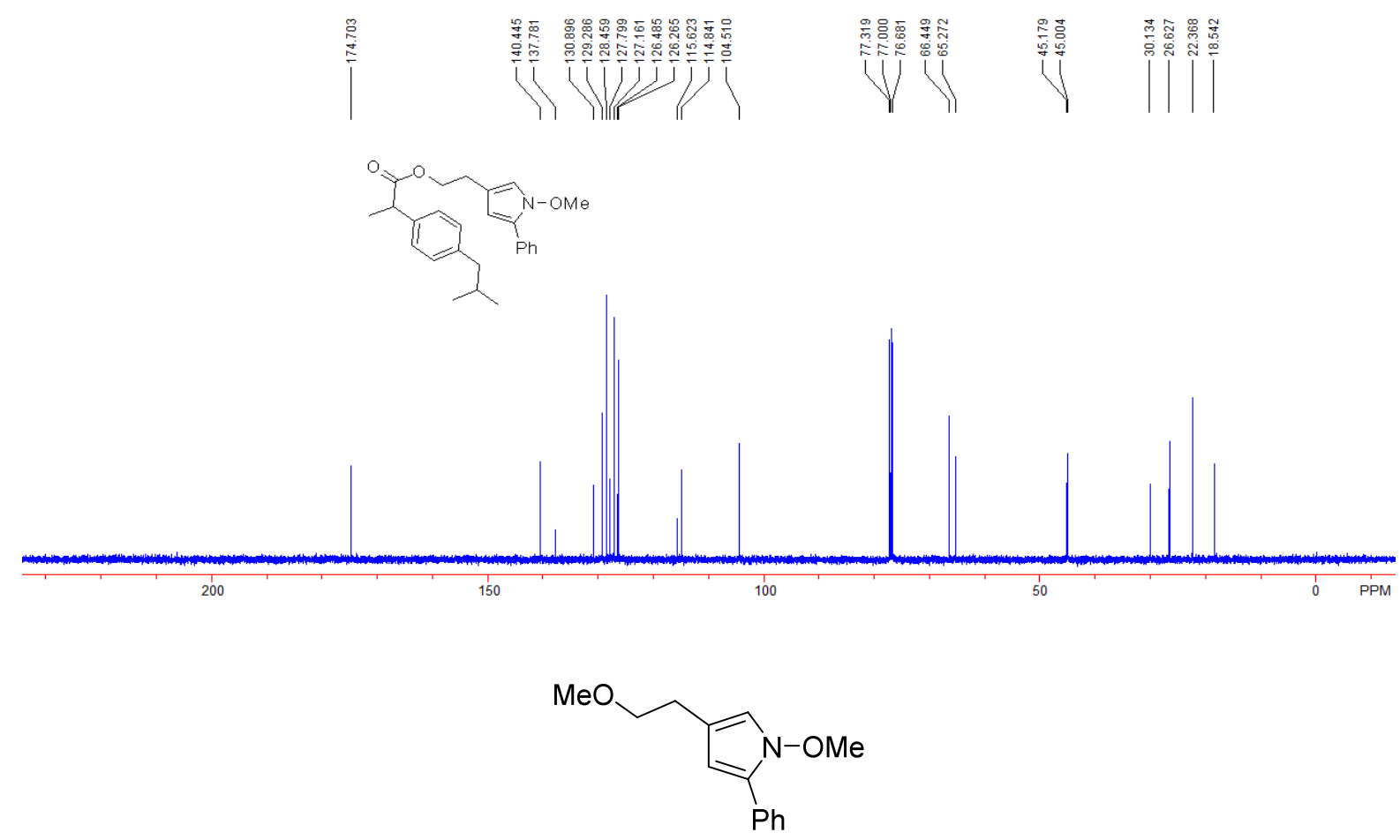

1-methoxy-4-(2-methoxyethyl)-2-phenyl-1H-pyrrole 3b: Yield: $86.0 \mathrm{mg}, 93 \%$; A colorless oil; ${ }^{1} \mathrm{H}$ NMR $\left(\mathrm{CDCl}_{3}, 400 \mathrm{MHz}, \mathrm{TMS}\right) \delta 2.74\left(\mathrm{t}, J=7.2 \mathrm{~Hz}, 2 \mathrm{H}, \mathrm{CH}_{2}\right), 3.39$ (s, 3H, $\left.\mathrm{CH}_{3}\right), 3.58$ (t, $J=7.2$ $\left.\mathrm{Hz}, 2 \mathrm{H}, \mathrm{CH}_{2}\right), 3.78\left(\mathrm{~s}, 3 \mathrm{H}, \mathrm{CH}_{3}\right), 6.11(\mathrm{~d}, J=2.0 \mathrm{~Hz}, 1 \mathrm{H}, \mathrm{Ar}), 6.74(\mathrm{~d}, J=2.0 \mathrm{~Hz}, 1 \mathrm{H}, \mathrm{Ar})$, 7.22-7.25 (m, 1H, Ar), 7.35-7.38 (m, 2H, Ar), 7.64-7.66 (m, 2H, Ar). ${ }^{13} \mathrm{C}$ NMR $\left(\mathrm{CDCl}_{3}, 100 \mathrm{MHz}\right.$, TMS) $\delta 27.6,58.6,66.5,73.5,104.4,114.7,116.6,126.2,126.4,127.7,128.4,131.0$. IR (EtOH) $v$ $3274,2928,1715,1651,1599,1571,1507,1448,1374,1261,1194,1113,1004,965,882,802,759$, 732, $698 \mathrm{~cm}^{-1}$. MS (ESI) $\mathrm{m} / z 232(\mathrm{M}+\mathrm{H})^{+}$. HRMS (ESI) calcd. for $\mathrm{C}_{14} \mathrm{H}_{18} \mathrm{NO}_{2}:$ 232.1332, Found: 232.1334 .
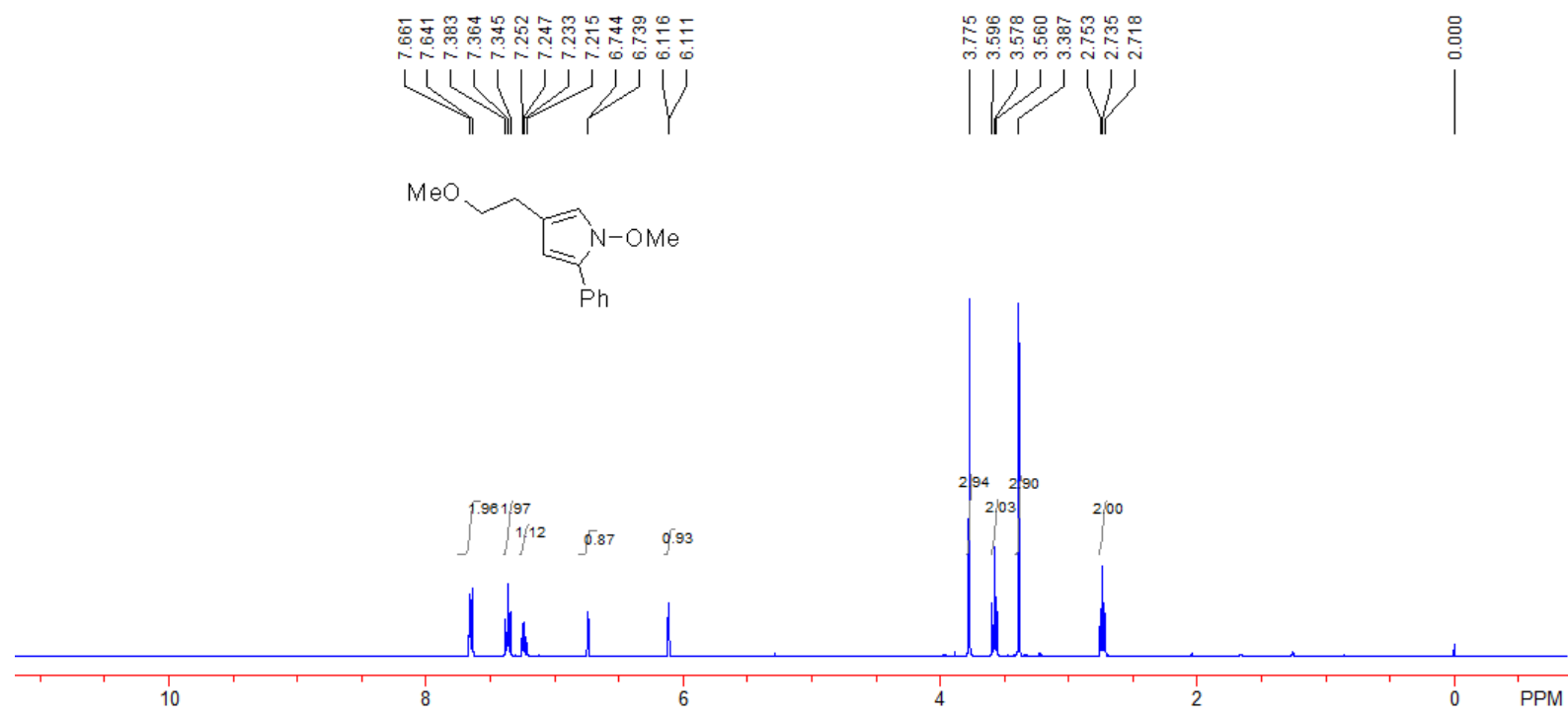

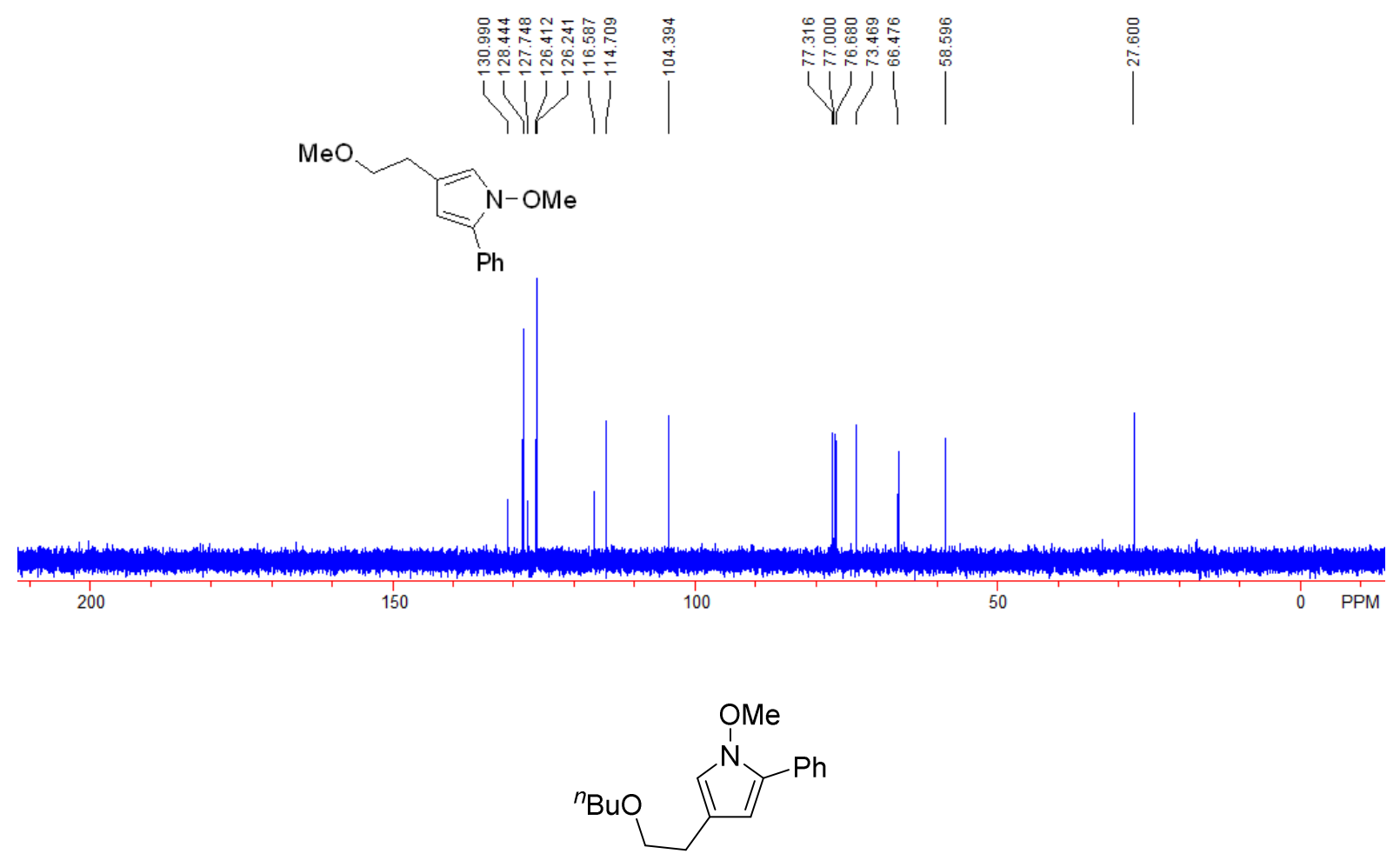

4-(2-butoxyethyl)-1-methoxy-2-phenyl-1H-pyrrole 3c: Yield: $61.2 \mathrm{mg}, 56 \%$; A colorless oil; ${ }^{1} \mathrm{H}$ NMR $\left(\mathrm{CDCl}_{3}, 400 \mathrm{MHz}\right.$, TMS) $\delta 0.93\left(\mathrm{t}, J=7.2 \mathrm{~Hz}, 3 \mathrm{H}, \mathrm{CH}_{3}\right), 1.36-1.42\left(\mathrm{~m}, 2 \mathrm{H}, \mathrm{CH}_{2}\right), 1.55-1.62$ (m, 2H, $\left.\mathrm{CH}_{2}\right), 2.73$ (t, J=7.2 Hz, 2H, $\left.\mathrm{CH}_{2}\right), 3.47$ (t, $\left.J=7.2 \mathrm{~Hz}, 2 \mathrm{H}, \mathrm{CH}_{2}\right), 3.60(\mathrm{t}, J=7.2 \mathrm{~Hz}, 2 \mathrm{H}$, $\left.\mathrm{CH}_{2}\right), 3.78\left(\mathrm{~s}, 3 \mathrm{H}, \mathrm{CH}_{3}\right), 6.11(\mathrm{~s}, 1 \mathrm{H}, \mathrm{Ar}), 6.74(\mathrm{~s}, 1 \mathrm{H}, \mathrm{Ar}), 7.23-7.26(\mathrm{~m}, 1 \mathrm{H}, \mathrm{Ar}), 7.35-7.38(\mathrm{~m}, 2 \mathrm{H}$, Ar), 7.64-7.66 (m, 2H, Ar). ${ }^{13} \mathrm{C} \mathrm{NMR} \mathrm{(} \mathrm{CDCl}_{3}, 100 \mathrm{MHz}$, TMS) $\delta$ 13.9, 19.4, 27.8, 31.8, 66.5, 70.7, 71.7, 104.6, 114.8, 116.8, 126.3, 126.4, 127.7, 128.5, 131.1. IR (EtOH) v 3057, 2995, 2961, 2936, 2899, 2868, 2811, 1719, 1598, 1490, 1464, 1442, 1105, 1058, 1010, 924, 897, 826, 793, 755, 691 $\mathrm{cm}^{-1}$. MS (ESI) $m / z 274(\mathrm{M}+\mathrm{H})^{+}$. HRMS (ESI) calcd. for $\mathrm{C}_{17} \mathrm{H}_{24} \mathrm{NO}_{2}$ : 274.1802, Found: 274.1803 .

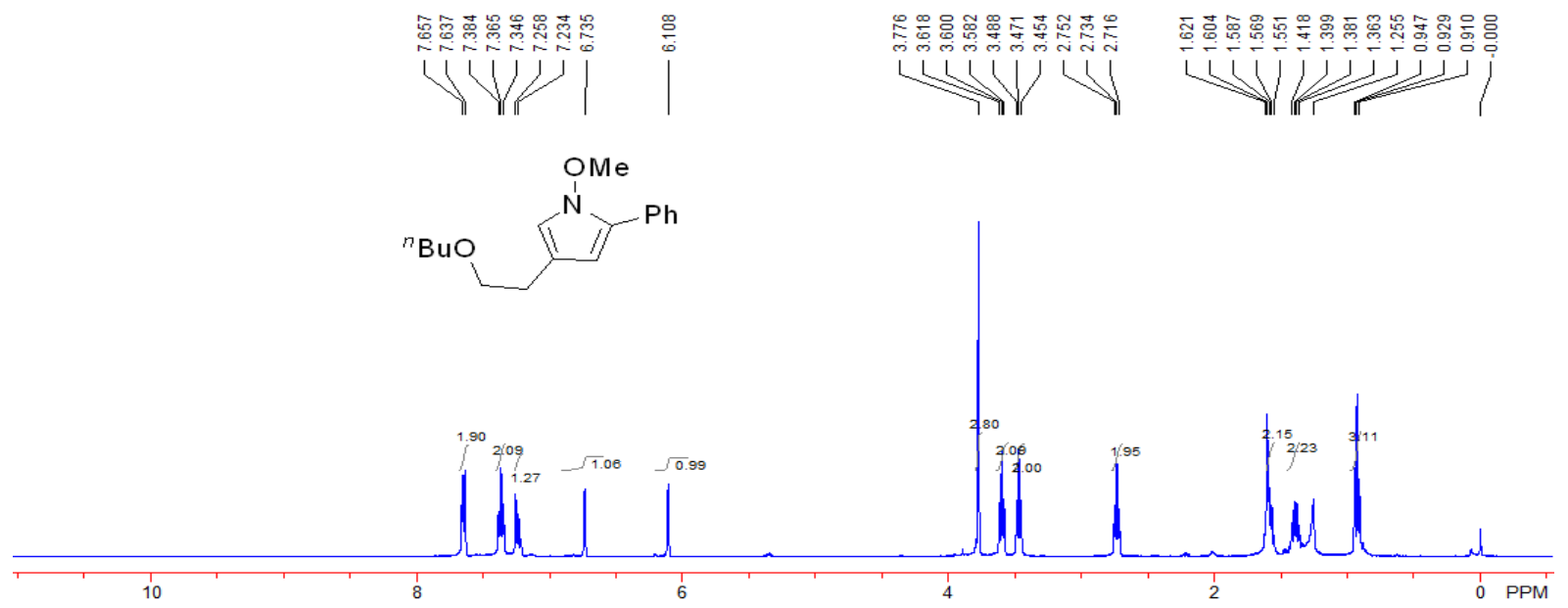



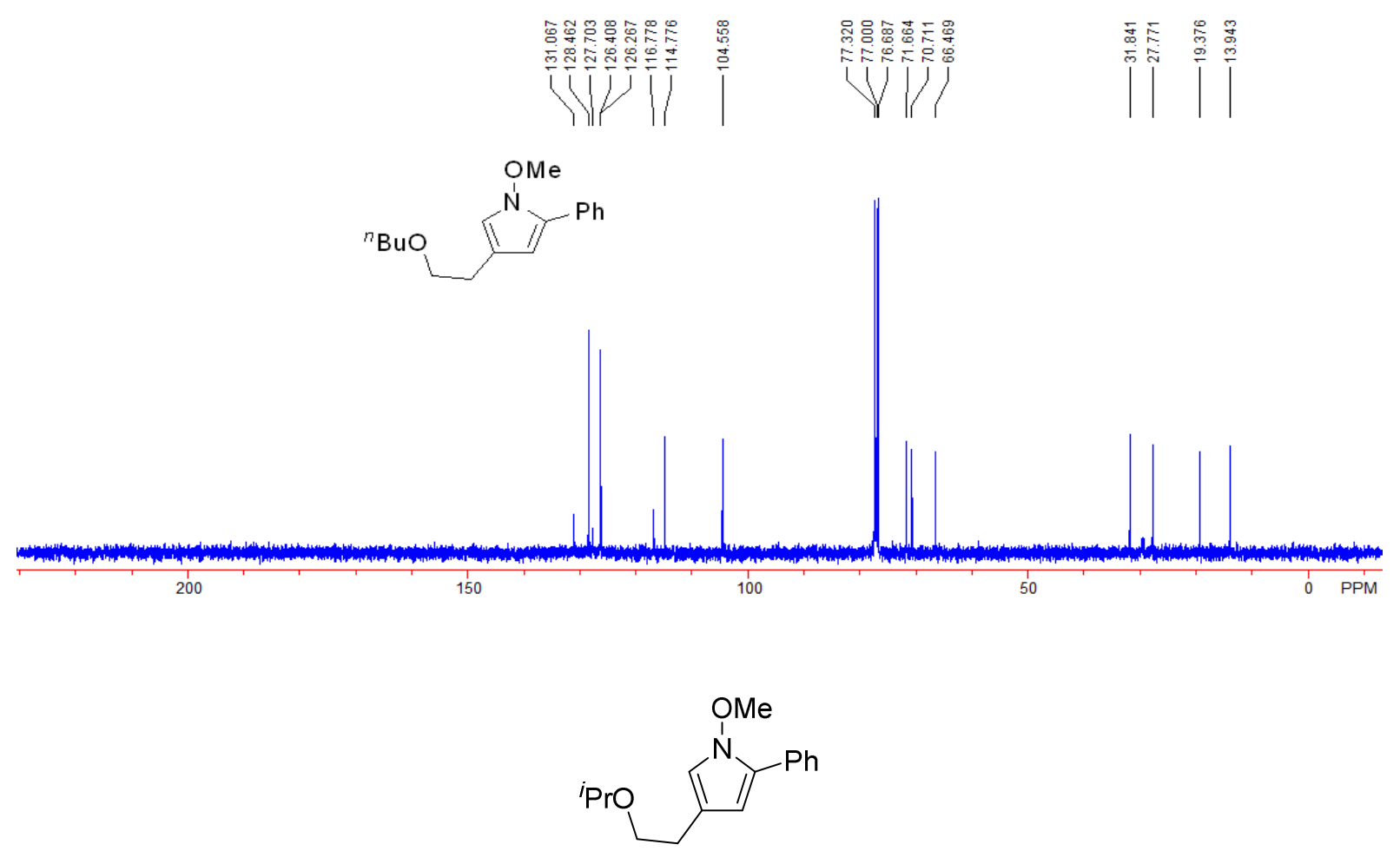

4-(2-isopropoxyethyl)-1-methoxy-2-phenyl-1H-pyrrole 3d: Yield: 46.8 mg, 45\%; A colorless oil;

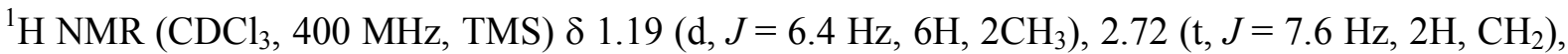
3.57-3.64 (m, 3H, $\left.\mathrm{CH}_{2}, \mathrm{CH}\right), 3.78\left(\mathrm{~s}, 3 \mathrm{H}, \mathrm{CH}_{3}\right), 6.11$ (d, $\left.J=2.4 \mathrm{~Hz}, 1 \mathrm{H}, \mathrm{Ar}\right), 6.74$ (d, $J=2.4 \mathrm{~Hz}$,

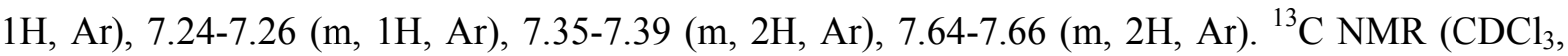
$100 \mathrm{MHz}, \mathrm{TMS}) \delta 22.2,28.2,66.5,69.1,71.4,104.6,114.8,116.8,126.2,126.4,127.7,128.5$, 131.0. IR $(\mathrm{EtOH}) \vee 2969,2929,2856,1717,1649,1599,1571,1509,1491,1447,1377,1368,1335$, $1261,1214,1174,1126,1077,1005,967,921,801,757,730,696 \mathrm{~cm}^{-1}$. MS (ESI) $\mathrm{m} / z 260(\mathrm{M}+\mathrm{H})^{+}$. HRMS (ESI) calcd. for $\mathrm{C}_{16} \mathrm{H}_{22} \mathrm{NO}_{2}: 260.1645$, Found: 260.1645.
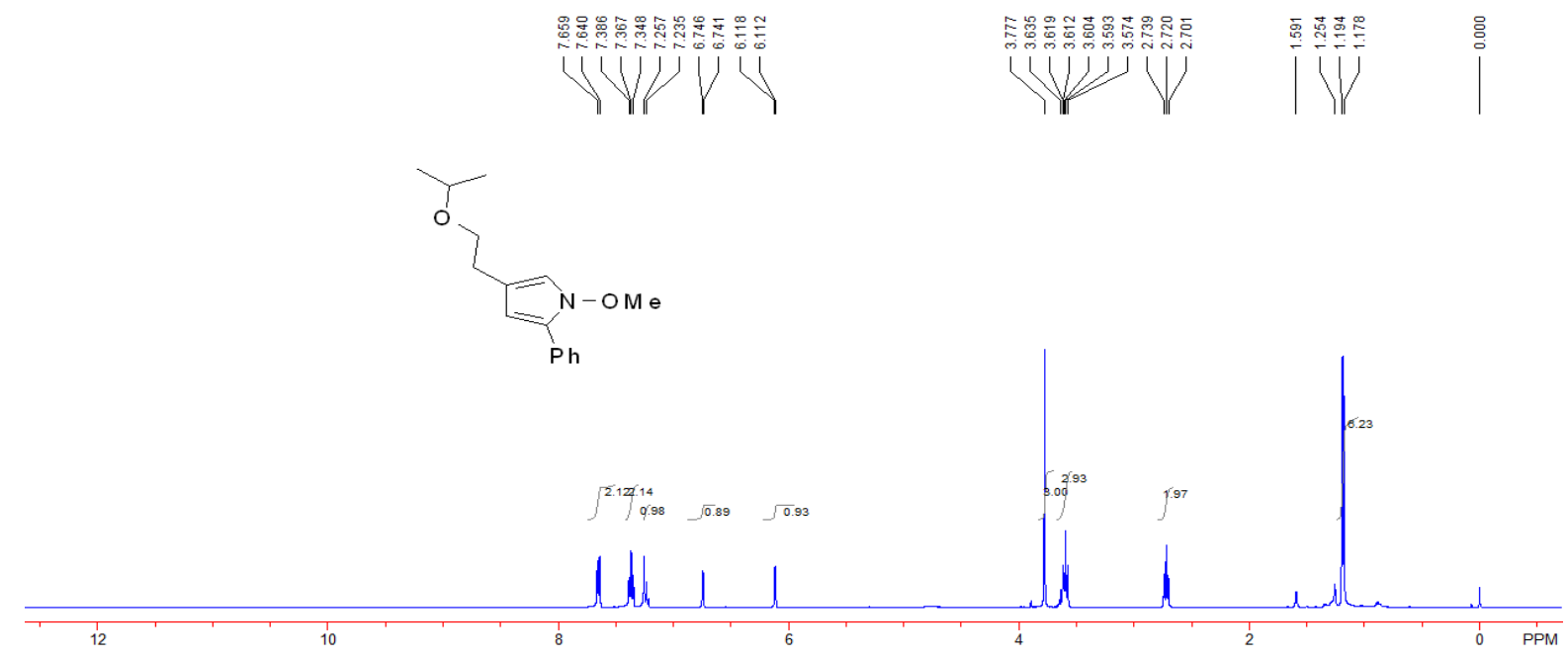


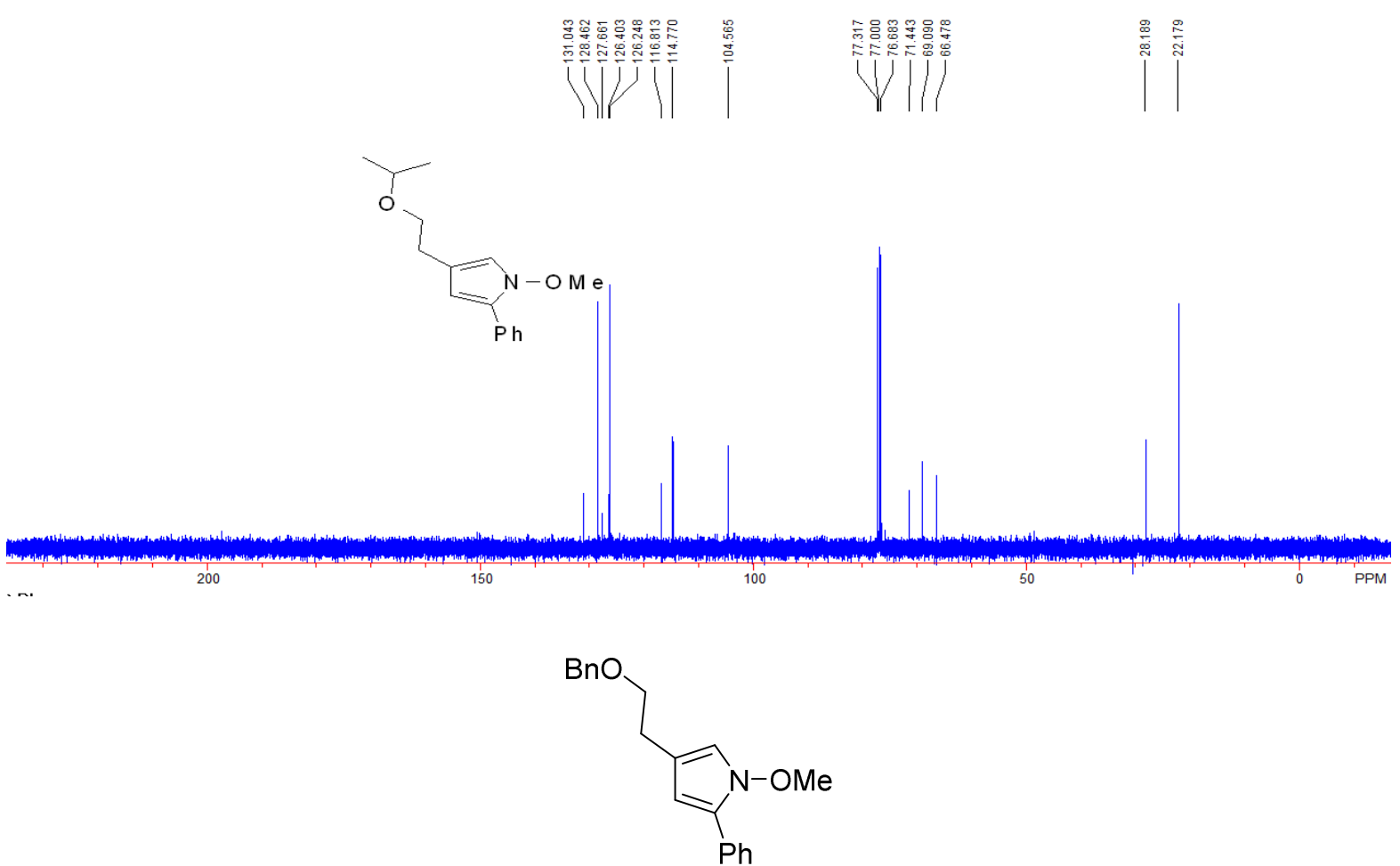

4-(2-(benzyloxy)ethyl)-1-methoxy-2-phenyl-1H-pyrrole 3e: Yield: $53.0 \mathrm{mg}$, 43\%; A colorless oil; ${ }^{1} \mathrm{H} \mathrm{NMR}\left(\mathrm{CDCl}_{3}, 400 \mathrm{MHz}, \mathrm{TMS}\right) \delta 2.78\left(\mathrm{t}, J=7.2 \mathrm{~Hz}, 2 \mathrm{H}, \mathrm{CH}_{2}\right), 3.67$ (t, $\left.J=7.2 \mathrm{~Hz}, 2 \mathrm{H}, \mathrm{CH}_{2}\right)$, $3.77\left(\mathrm{~s}, 3 \mathrm{H}, \mathrm{CH}_{3}\right), 4.57\left(\mathrm{~s}, 2 \mathrm{H}, \mathrm{CH}_{2}\right), 6.11(\mathrm{~d}, J=2.4 \mathrm{~Hz}, 1 \mathrm{H}, \mathrm{Ar}), 6.74(\mathrm{~d}, J=2.4 \mathrm{~Hz}, 1 \mathrm{H}, \mathrm{Ar})$, 7.24-7.29 (m, 2H, Ar), 7.35-7.39 (m, 6H, Ar), 7.64-7.65 (m, 2H, Ar). ${ }^{13} \mathrm{C}$ NMR $\left(\mathrm{CDCl}_{3}, 100 \mathrm{MHz}\right.$, TMS) $\delta 27.8,66.5,71.1,72.9,104.6,114.8,116.6,126.3,126.4,127.5,127.7,128.3,128.5,129.6$, 131.0, 138.5. IR $(\mathrm{EtOH}) \vee 3062,3028,2933,2855,1717,1601,1508,1494,1453,1361,1271$, 1210, 1099, 1060, 1027, 1005, 994, 966, 798, 757, $695 \mathrm{~cm}^{-1}$. MS (ESI) $m / z 308(\mathrm{M}+\mathrm{H})^{+}$. HRMS (ESI) calcd. for $\mathrm{C}_{20} \mathrm{H}_{22} \mathrm{NO}_{2}$ : 308.1645, Found: 308.1646 .
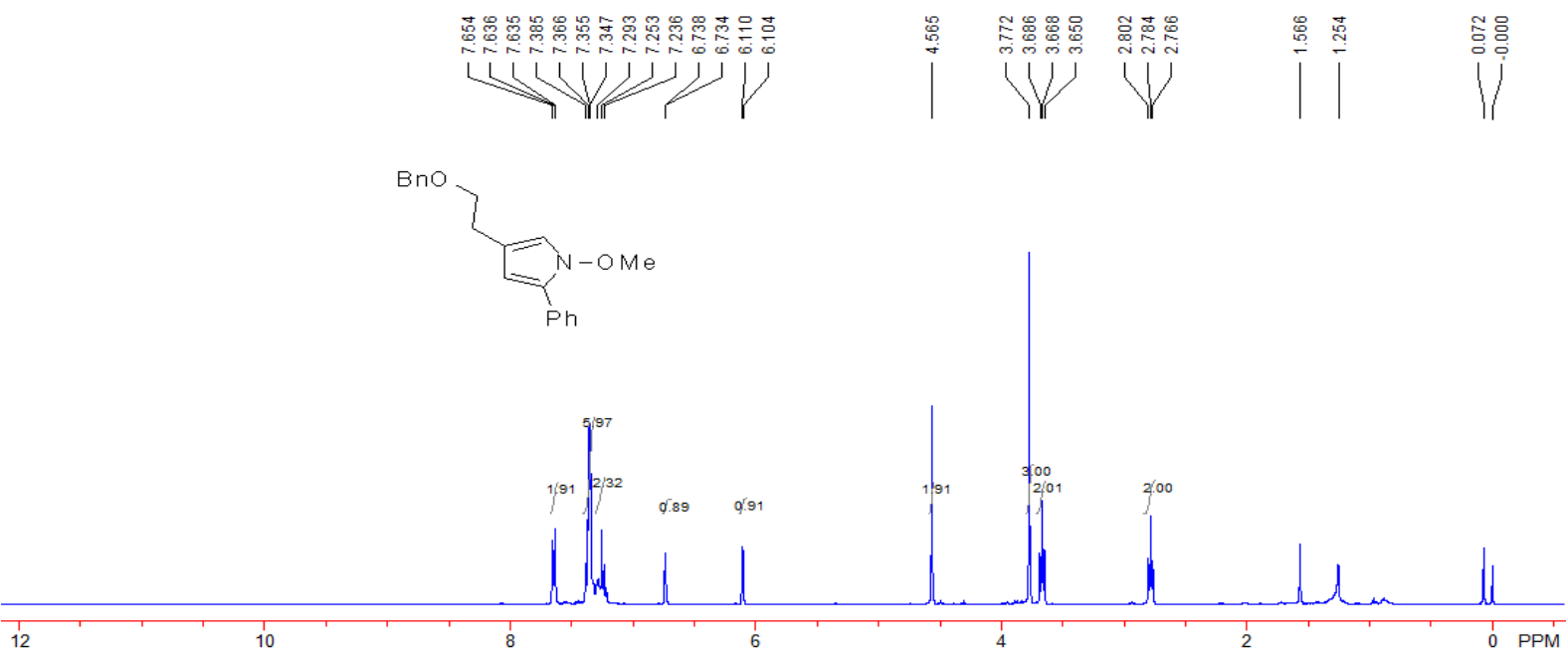

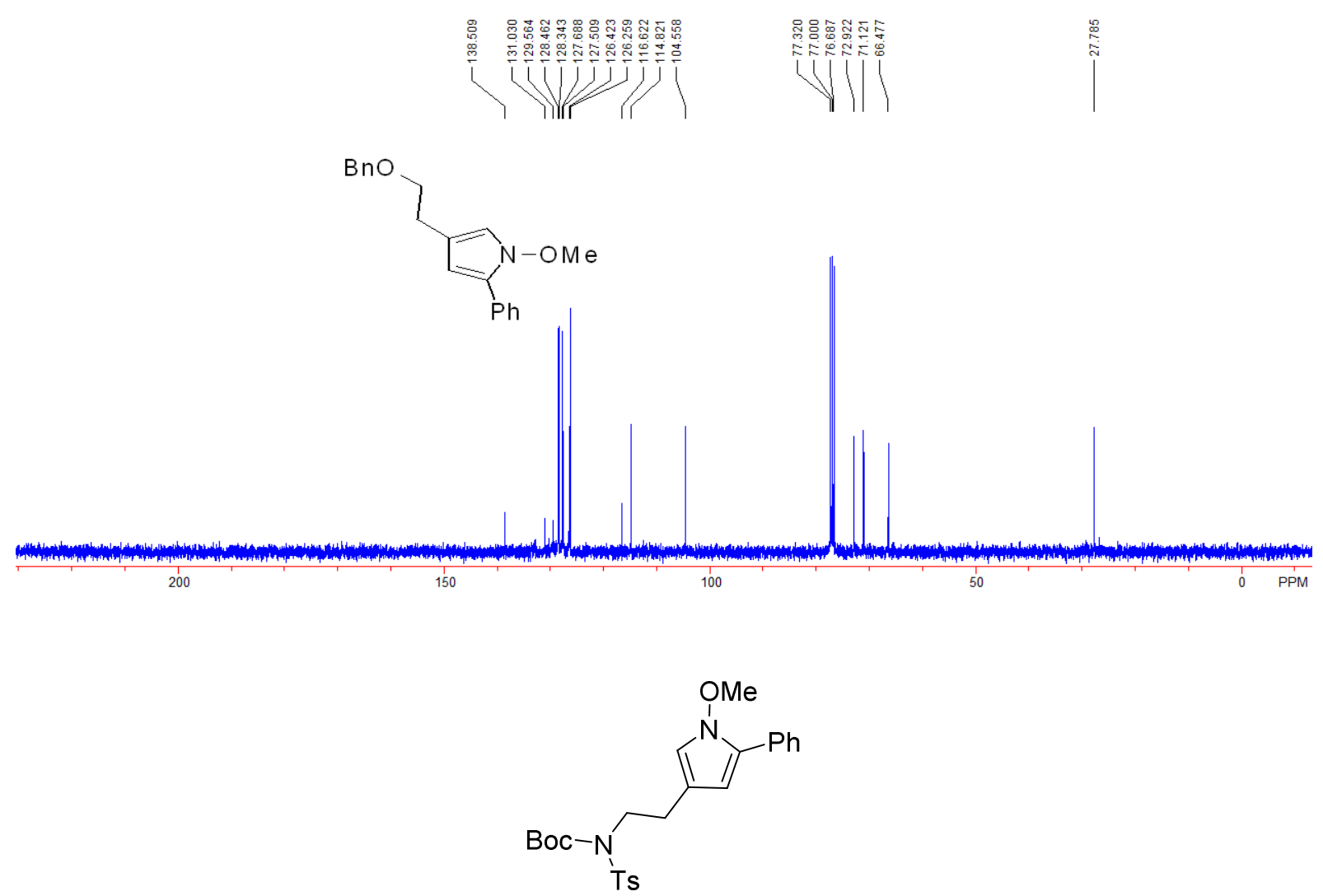

tert-butyl (2-(1-methoxy-5-phenyl-1H-pyrrol-3-yl)ethyl)(tosyl)carbamate 3f: Yield: $164.8 \mathrm{mg}, 88 \%$; A colorless oil; ${ }^{1} \mathrm{H}$ NMR $\left(\mathrm{CDCl}_{3}, 400 \mathrm{MHz}, \mathrm{TMS}\right) \delta 1.34\left(\mathrm{~s}, 9 \mathrm{H}, 3 \mathrm{CH}_{3}\right), 2.42\left(\mathrm{~s}, 3 \mathrm{H}, \mathrm{CH}_{3}\right), 2.90(\mathrm{t}$, $\left.J=8.0 \mathrm{~Hz}, 2 \mathrm{H}, \mathrm{CH}_{2}\right), 3.78\left(\mathrm{~s}, 3 \mathrm{H}, \mathrm{CH}_{3}\right), 3.98-4.02\left(\mathrm{t}, J=8.0 \mathrm{~Hz}, 2 \mathrm{H}, \mathrm{CH}_{2}\right), 6.14(\mathrm{~d}, J=2.4 \mathrm{~Hz}, 1 \mathrm{H}$, Ar), 6.76 (d, $J=2.4 \mathrm{~Hz}, 1 \mathrm{H}, \mathrm{Ar}), 7.24-7.29$ (m, 3H, Ar), 7.35-7.39 (m, 2H, Ar), 7.65 (d, $J=8.0 \mathrm{~Hz}$, 2H, Ar), 7.78 (d, $J=8.0 \mathrm{~Hz}, 2 \mathrm{H}, \mathrm{Ar}) .{ }^{13} \mathrm{C} \mathrm{NMR}\left(\mathrm{CDCl}_{3}, 100 \mathrm{MHz}, \mathrm{TMS}\right) \delta 21.5,27.8,28.0,48.3$, $66.5,84.0,104.6,115.0,115.9,126.2$, 126.5, 127.8, 127.9, 128.5, 129.2, 130.9, 137.4, 144.0, 150.9. IR $(\mathrm{EtOH}) \vee 2978,2253,1724,1598,1508,1446,1350,1289,1256,1256,1152,1087,1063,966$, 909, 849, 812, $760 \mathrm{~cm}^{-1}$. MS (ESI) $\mathrm{m} / z 471(\mathrm{M}+\mathrm{H})^{+}$. HRMS (ESI) calcd. for $\mathrm{C}_{25} \mathrm{H}_{31} \mathrm{~N}_{2} \mathrm{O}_{5} \mathrm{~S}$ : 471.1948, Found: 471.1952. 


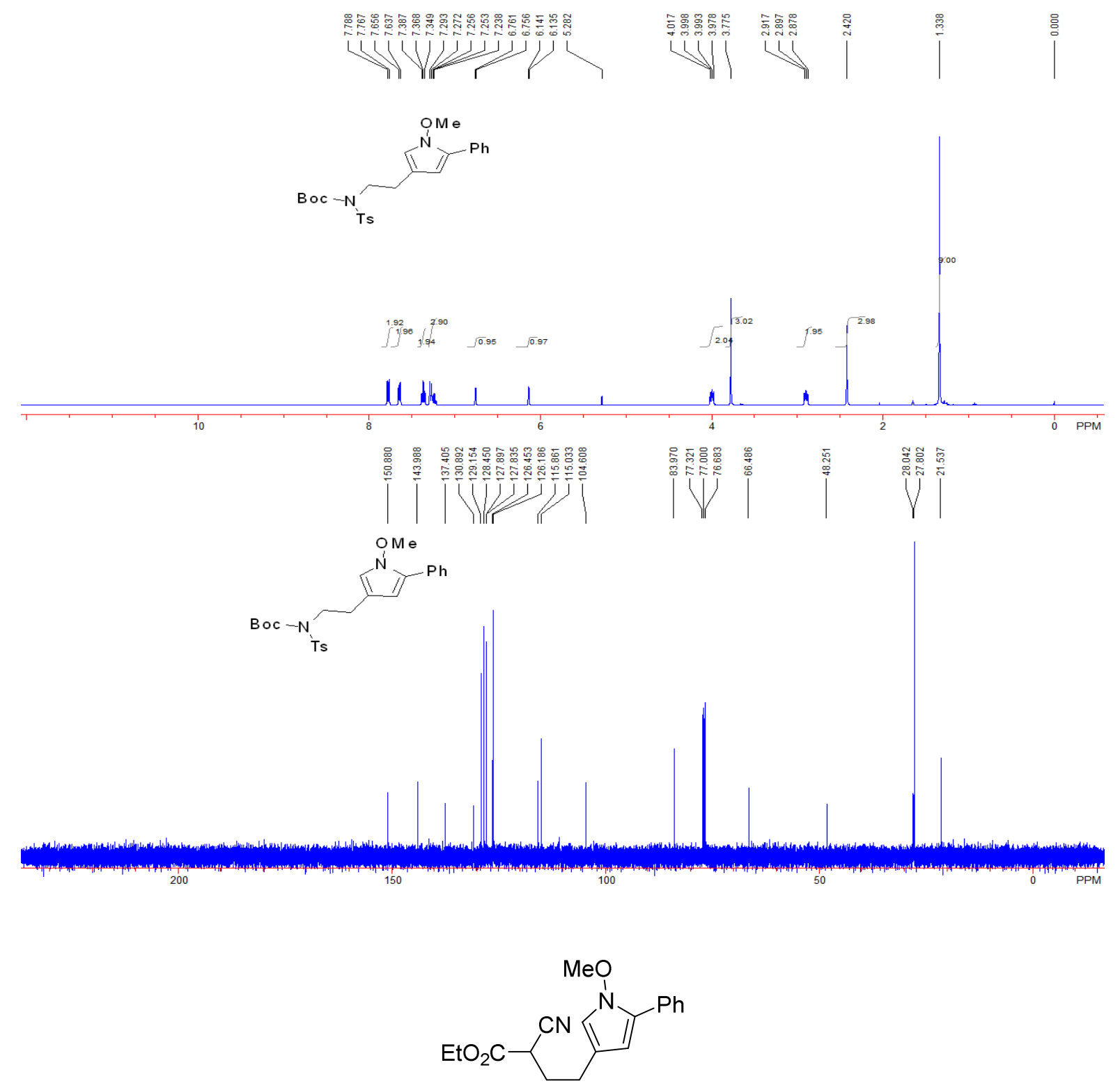

ethyl 2-cyano-4-(1-methoxy-5-phenyl-1H-pyrrol-3-yl)butanoate 3g: Yield: $92.6 \mathrm{mg}, 74 \%$; A colorless oil; ${ }^{1} \mathrm{H}$ NMR $\left(\mathrm{CDCl}_{3}, 400 \mathrm{MHz}, \mathrm{TMS}\right) \delta 1.31$ (t, $\left.J=7.2 \mathrm{~Hz}, 3 \mathrm{H}, \mathrm{CH}_{3}\right), 2.18-2.25$ (m, 2H, $\left.\mathrm{CH}_{2}\right), 2.62-2.78\left(\mathrm{~m}, 2 \mathrm{H}, \mathrm{CH}_{2}\right), 3.53-3.56(\mathrm{~m}, 1 \mathrm{H}, \mathrm{CH}), 3.79\left(\mathrm{~s}, 3 \mathrm{H}, \mathrm{CH}_{3}\right), 4.24(\mathrm{q}, J=7.2 \mathrm{~Hz}, 2 \mathrm{H}$, $\left.\mathrm{CH}_{2}\right), 6.09$ (d, $\left.J=2.0 \mathrm{~Hz}, 1 \mathrm{H}, \mathrm{Ar}\right), 6.74(\mathrm{~m}, J=2.0 \mathrm{~Hz}, 1 \mathrm{H}, \mathrm{Ar}), 7.24-7.28$ (m, 1H, Ar), 7.36-7.40 (m, 2H, Ar), 7.64-7.66 (m, 2H, Ar). ${ }^{13} \mathrm{C} \mathrm{NMR}\left(\mathrm{CDCl}_{3}, 100 \mathrm{MHz}, \mathrm{TMS}\right) \delta$ 14.0, 24.1, 31.0, 36.6, $62.7,66.6,104.0,114.8,116.5,116.7,126.3,126.7,128.4,128.6,130.7,166.3$. IR $($ EtOH) $\vee 2964$, 2924, 2853, 2249, 1741, 1602, 1509, 1444, 1369, 1252, 1198, 1126, 1095, 1019, 1005, 995, 965, 913, 854, 801, 760, 695, $666 \mathrm{~cm}^{-1}$. MS (ESI) $\mathrm{m} / z 313(\mathrm{M}+\mathrm{H})^{+}$. HRMS (ESI) calcd. for $\mathrm{C}_{18} \mathrm{H}_{21} \mathrm{~N}_{2} \mathrm{O}_{3}$ : 313.1547, Found: 313.1548. 


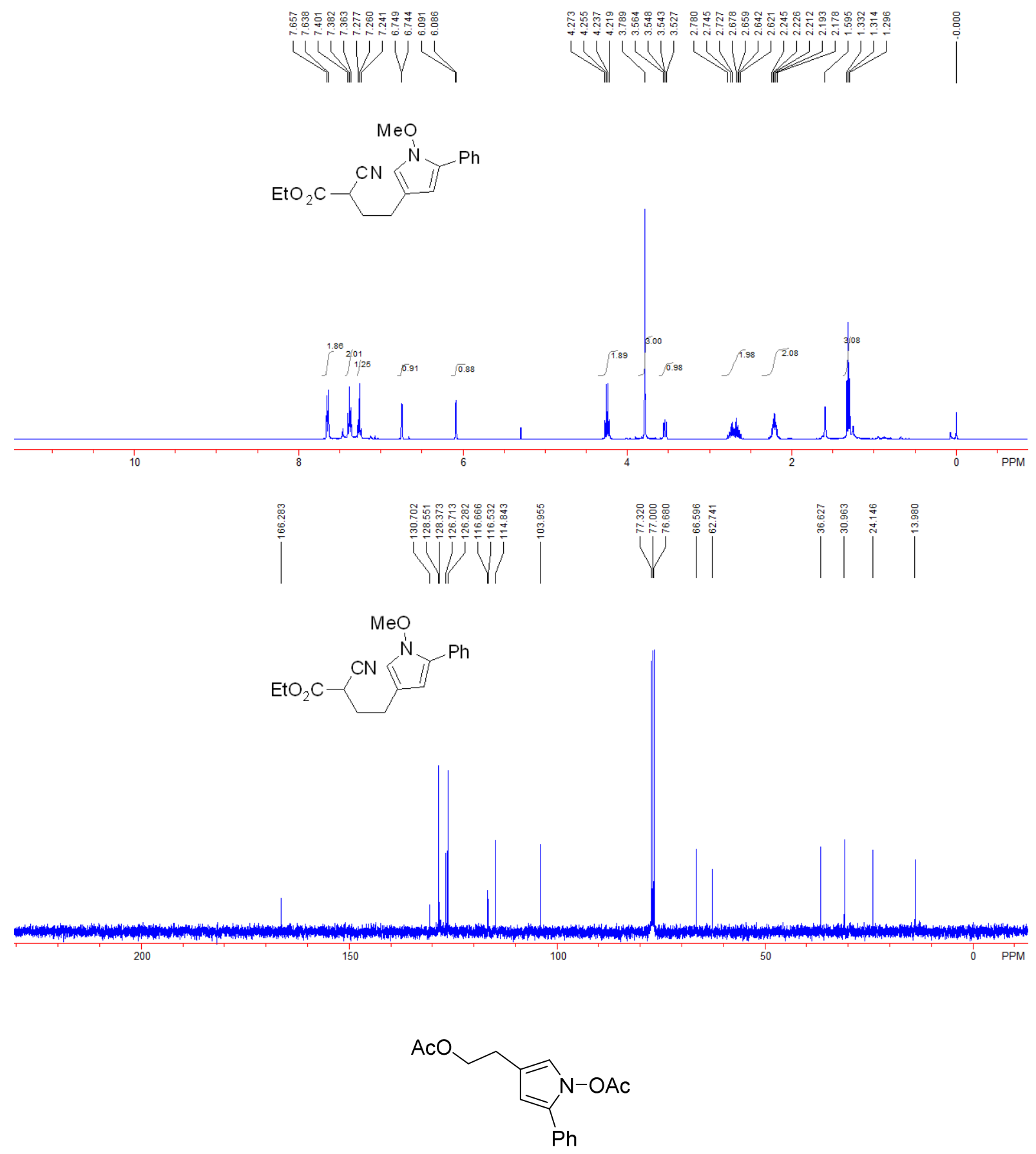

2-(1-acetoxy-5-phenyl-1H-pyrrol-3-yl)ethyl acetate 5a: Yield: $37.2 \mathrm{mg}, 65 \%$; A colorless oil; ${ }^{1} \mathrm{H}$ NMR $\left(\mathrm{CDCl}_{3}, 400 \mathrm{MHz}, \mathrm{TMS}\right) \delta 2.06\left(\mathrm{~s}, 3 \mathrm{H}, \mathrm{CH}_{3}\right), 2.17\left(\mathrm{~s}, 3 \mathrm{H}, \mathrm{CH}_{3}\right), 2.81(\mathrm{t}, J=7.2 \mathrm{~Hz}, 2 \mathrm{H}$, $\left.\mathrm{CH}_{2}\right), 4.26\left(\mathrm{t}, J=7.2 \mathrm{~Hz}, 2 \mathrm{H}, \mathrm{CH}_{2}\right), 6.17(\mathrm{~d}, J=2.0 \mathrm{~Hz}, 1 \mathrm{H}, \mathrm{Ar}), 6.64$ (d, $\left.J=2.0 \mathrm{~Hz}, 1 \mathrm{H}, \mathrm{Ar}\right)$, 7.23-7.27 (m, 1H, Ar), 7.33-7.37 (m, 2H, Ar), 7.43-7.45 (m, 1H, Ar. ${ }^{13} \mathrm{C}$ NMR $\left(\mathrm{CDCl}_{3}, 100 \mathrm{MHz}\right.$, TMS) $\delta 18.1,21.0,26.6,64.7,105.2,116.4,116.9,126.7,127.0,128.5,130.4,130.8,168.6,171.1$. IR $(\mathrm{EtOH}) \vee$ 3059, 3028, 2956, 2933, 2855, 1808, 1734, 1604, 1512, 1491, 1365, 1239, 1165, 1032 , 
1004, 905, 843, 758, $695 \mathrm{~cm}^{-1}$. MS (ESI) $\mathrm{m} / z 288(\mathrm{M}+\mathrm{H})^{+}$. HRMS (ESI) calcd. for $\mathrm{C}_{16} \mathrm{H}_{18} \mathrm{NO}_{4}$ : 288.1230, Found: 288.1232.
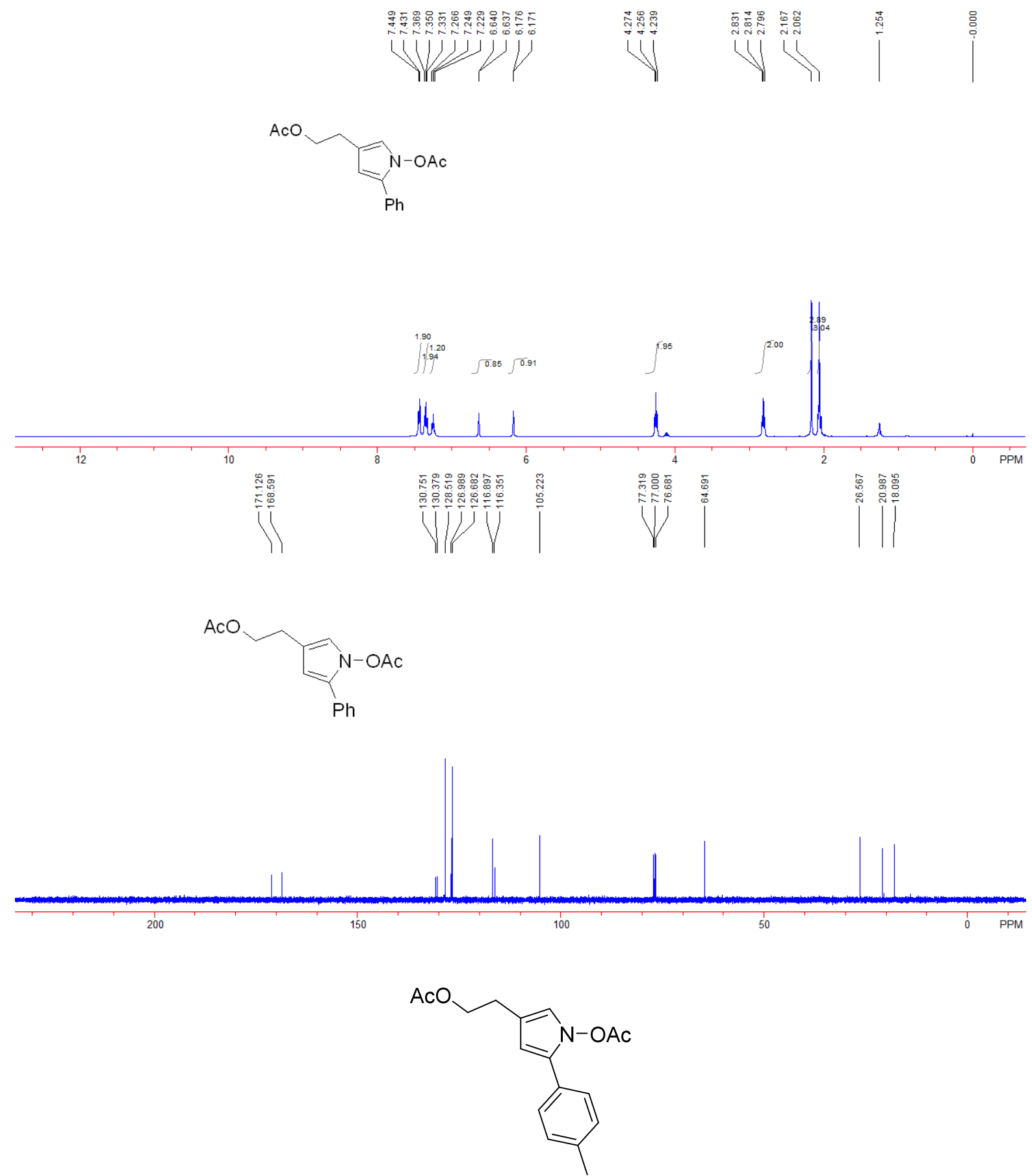

2-(1-acetoxy-5-(p-tolyl)-1H-pyrrol-3-yl)ethyl acetate 5b: Yield: $43.4 \mathrm{mg}, 68 \%$; A colorless oil; ${ }^{1} \mathrm{H}$ NMR $\left(\mathrm{CDCl}_{3}, 400 \mathrm{MHz}, \mathrm{TMS}\right) \delta 2.06\left(\mathrm{~s}, 3 \mathrm{H}, \mathrm{CH}_{3}\right), 2.18\left(\mathrm{~s}, 3 \mathrm{H}, \mathrm{CH}_{3}\right), 2.35\left(\mathrm{~s}, 3 \mathrm{H}, \mathrm{CH}_{3}\right), 2.81(\mathrm{t}, J$ $\left.=7.2 \mathrm{~Hz}, 2 \mathrm{H}, \mathrm{CH}_{2}\right), 4.25\left(\mathrm{t}, J=7.2 \mathrm{~Hz}, 2 \mathrm{H}, \mathrm{CH}_{2}\right), 6.13$ (d, J=2.0 Hz, 1H, Ar), 6.62 (d, $J=2.0 \mathrm{~Hz}$, 1H, Ar), 7.16 (d, $J=8.0 \mathrm{~Hz}, 2 \mathrm{H}, \mathrm{Ar}), 7.33$ (d, $J=8.0 \mathrm{~Hz}, 2 \mathrm{H}, \mathrm{Ar}) .{ }^{13} \mathrm{C} \mathrm{NMR}\left(\mathrm{CDCl}_{3}, 100 \mathrm{MHz}\right.$, 
TMS) $\delta 18.2,21.0,21.2,26.6,64.7,104.8,116.3,116.5,126.7,127.6,129.3,130.9,136.8,168.7$, 171.1. IR $(\mathrm{EtOH}) \vee 3358,3191,2919,2849,1797,1728,1658,1632,1524,1469,1426,1366,1243$ $1165,1033,1002,923,879,87,820,791,773,721 \mathrm{~cm}^{-1}$. MS (ESI) $m / z 302(\mathrm{M}+\mathrm{H})^{+}$. HRMS (ESI) calcd. for $\mathrm{C}_{17} \mathrm{H}_{20} \mathrm{NO}_{4}$ : 302.1387, Found: 302.1388 .
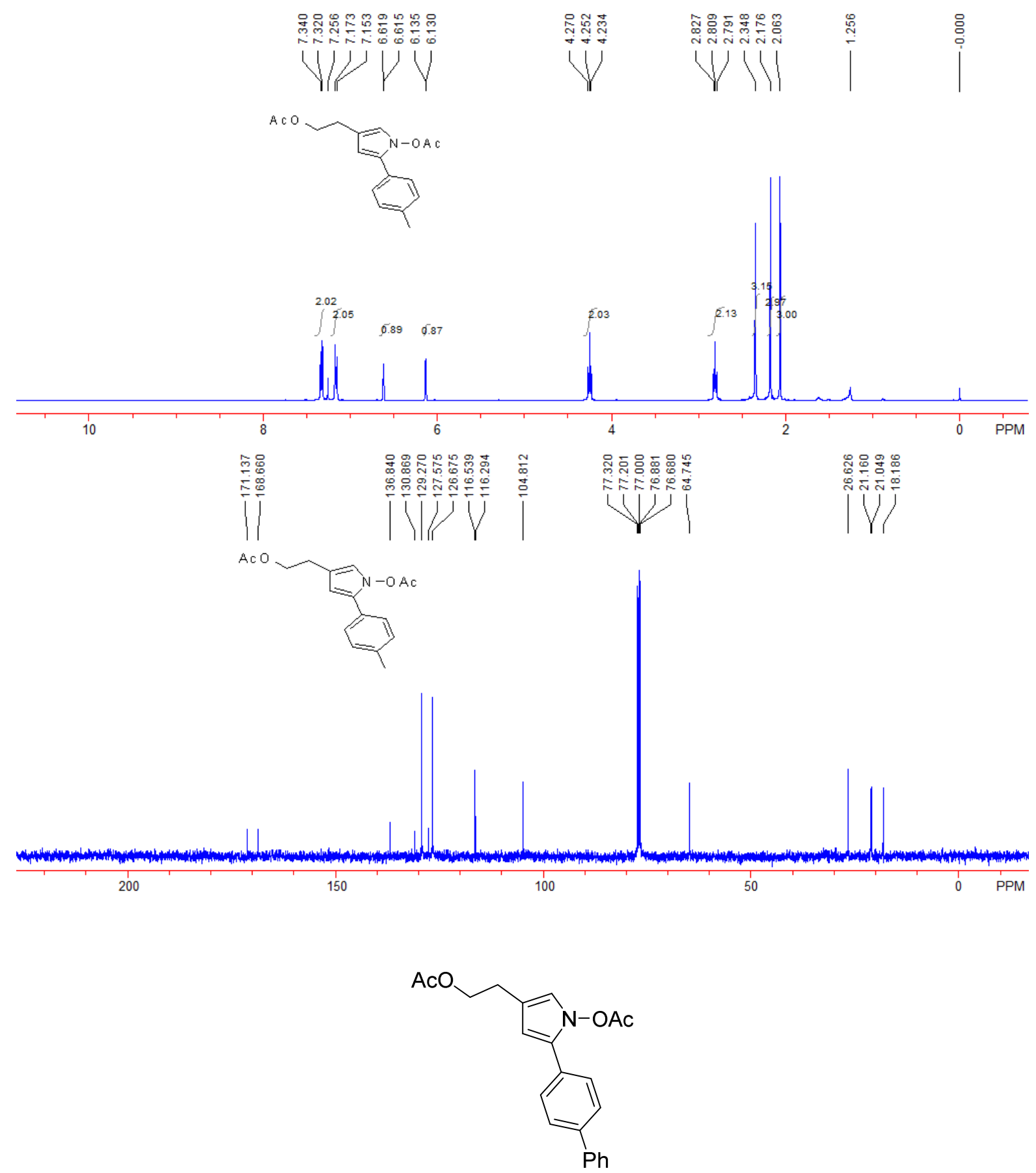

2-(5-([1,1'-biphenyl]-4-yl)-1-acetoxy-1H-pyrrol-3-yl)ethyl acetate 5c: Yield: $39.6 \mathrm{mg}$, 55\%; A colorless oil; ${ }^{1} \mathrm{H}$ NMR $\left(\mathrm{CDCl}_{3}, 400 \mathrm{MHz}, \mathrm{TMS}\right) \delta 2.08\left(\mathrm{~s}, 3 \mathrm{H}, \mathrm{CH}_{3}\right), 2.22\left(\mathrm{~s}, 3 \mathrm{H}, \mathrm{CH}_{3}\right), 2.83(\mathrm{t}, J=$ 
$\left.7.2 \mathrm{~Hz}, 2 \mathrm{H}, \mathrm{CH}_{2}\right), 4.27$ (t, $\left.J=7.2 \mathrm{~Hz}, 2 \mathrm{H}, \mathrm{CH}_{2}\right), 6.23(\mathrm{~d}, J=2.0 \mathrm{~Hz}, 1 \mathrm{H}, \mathrm{Ar}), 6.66$ (d, $J=2.0 \mathrm{~Hz}$, 1H, Ar), 7.33-7.37 (m, 1H, Ar), 7.43-7.46 (m, 2H, Ar), 7.51-7.53 (m, 2H, Ar), 7.59-7.62 (m, 4H, Ar). ${ }^{13} \mathrm{C}$ NMR $\left(\mathrm{CDCl}_{3}, 100 \mathrm{MHz}, \mathrm{TMS}\right) \delta 18.2,21.1,26.6,29.7,64.7,105.4,116.5,117.1,126.9$, 127.0, 127.3, 127.4, 128.8, 129.4, 130.5, 139.7, 140.5, 168.7, 171.2. IR (EtOH) v 3057, 3026, 2921, 2852, 1808, 1734, 1600, 1527, 1494, 1470, 1419, 1365, 1239, 1194, 1164, 1124, 1033, 1005, 989, 840, 800, 764, 726, 698, $671 \mathrm{~cm}^{-1}$. MS (ESI) $\mathrm{m} / z 364(\mathrm{M}+\mathrm{H})^{+}$. HRMS (ESI) calcd. for $\mathrm{C}_{22} \mathrm{H}_{22} \mathrm{NO}_{4}$ : 364.1543, Found: 364.1546.
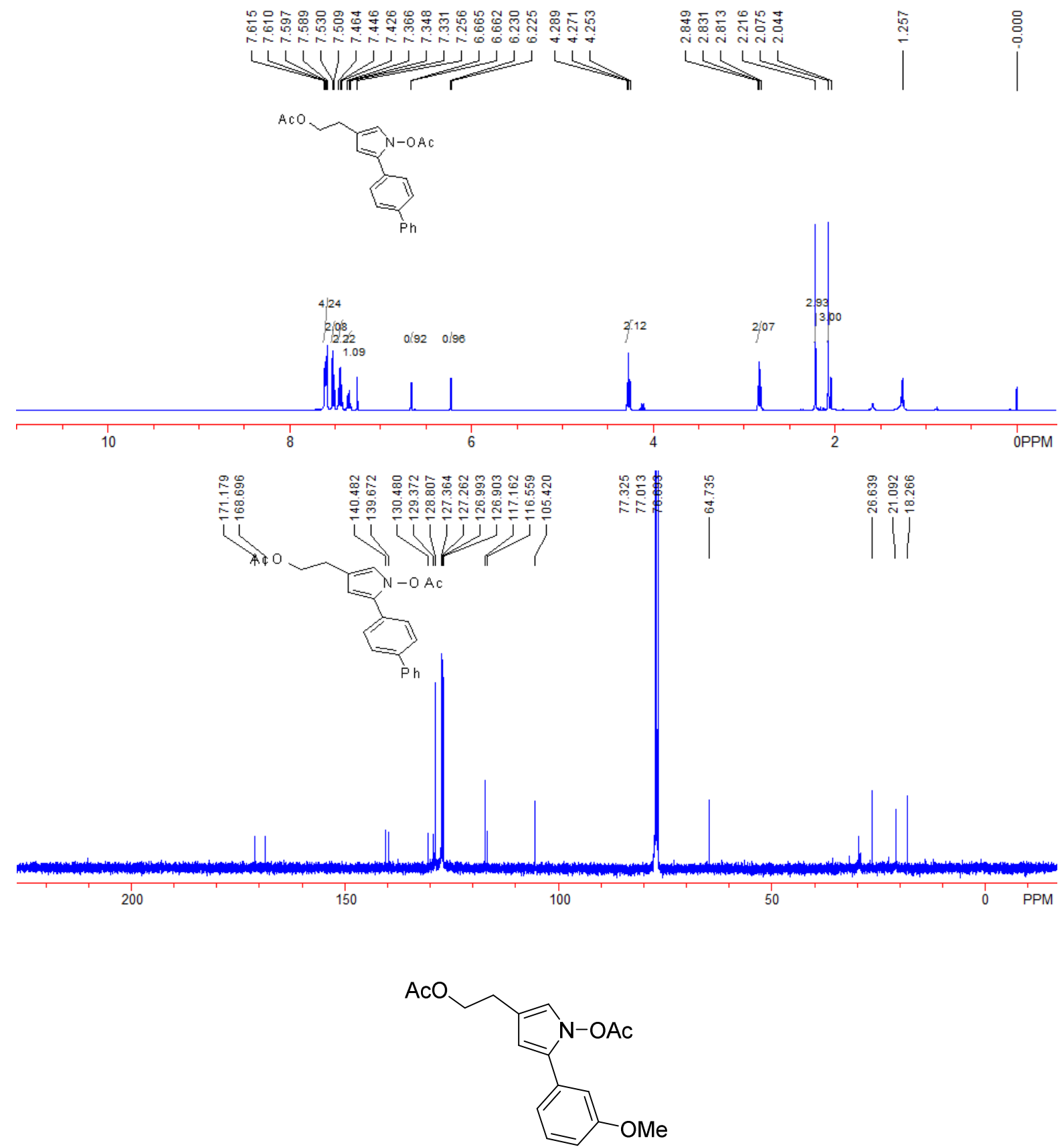
2-(1-acetoxy-5-(3-methoxyphenyl)-1H-pyrrol-3-yl)ethyl acetate 5d: Yield: $34.0 \mathrm{mg}, 54 \%$; A colorless oil; ${ }^{1} \mathrm{H}$ NMR $\left(\mathrm{CDCl}_{3}, 400 \mathrm{MHz}, \mathrm{TMS}\right) \delta 2.06\left(\mathrm{~s}, 3 \mathrm{H}, \mathrm{CH}_{3}\right), 2.20\left(\mathrm{~s}, 3 \mathrm{H}, \mathrm{CH}_{3}\right), 2.81(\mathrm{t}, J=$ $\left.7.2 \mathrm{~Hz}, 2 \mathrm{H}, \mathrm{CH}_{2}\right), 3.81\left(\mathrm{~s}, 3 \mathrm{H}, \mathrm{CH}_{3}\right), 4.25$ (t, $\left.J=7.2 \mathrm{~Hz}, 2 \mathrm{H}, \mathrm{CH}_{2}\right), 6.18(\mathrm{~d}, J=2.0 \mathrm{~Hz}, 1 \mathrm{H}, \mathrm{Ar})$, $6.63(\mathrm{~d}, J=2.0 \mathrm{~Hz}, 1 \mathrm{H}, \mathrm{Ar}), 6.80-6.82$ (m, 1H, Ar), 6.98-6.99 (m, 1H, Ar), 7.03-7.05 (m, 1H, Ar), 7.25-7.29 (m, 1H, Ar). ${ }^{13} \mathrm{C}$ NMR ( $\mathrm{CDCl}_{3}, 100 \mathrm{MHz}$, TMS) $\delta$ 18.2, 21.0, 26.6, 55.2, 64.7, 105.5, 112.2, 112.7, 116.4, 117.0, 119.1, 129.6, 130.6, 131.6, 159.6, 168.6, 171.2. IR (EtOH) v 3124, 3008, 2953, 2934, 2834, 1807, 1735, 1607, 1581, 1510, 1481, 1466, 1365, 1285. 1240, 1197, 1161, 1123, 1037, 991, 942, 851, 818, 780, $693 \mathrm{~cm}^{-1}$. MS (ESI) $\mathrm{m} / z 318(\mathrm{M}+\mathrm{H})^{+}$. HRMS (ESI) calcd. for $\mathrm{C}_{17} \mathrm{H}_{20} \mathrm{NO}_{5}$ : 318.1336, Found: 318.1336 .

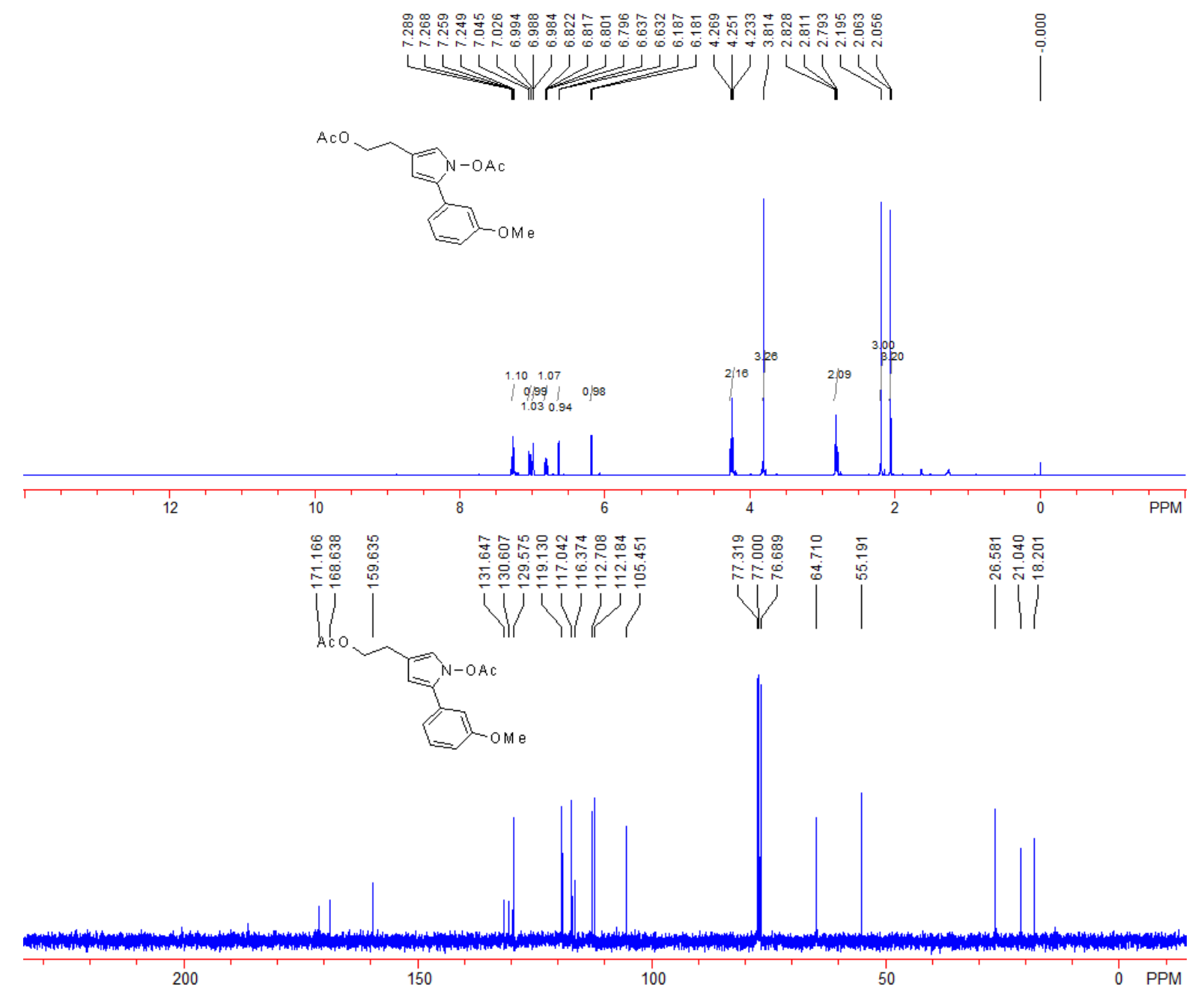




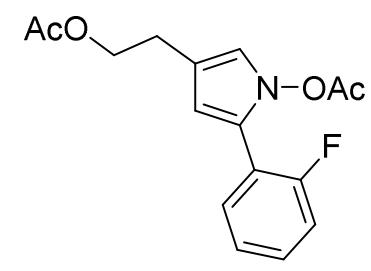

2-(1-acetoxy-5-(2-fluorophenyl)-1H-pyrrol-3-yl)ethyl acetate 5e: Yield: $32.8 \mathrm{mg}$, 54\%; A colorless oil; ${ }^{1} \mathrm{H}$ NMR $\left(\mathrm{CDCl}_{3}, 400 \mathrm{MHz}, \mathrm{TMS}\right) \delta 2.07$ (s, 3H, $\left.\mathrm{CH}_{3}\right), 2.16\left(\mathrm{~s}, 3 \mathrm{H}, \mathrm{CH}_{3}\right), 2.83$ (t, $J=7.2 \mathrm{~Hz}$, $\left.2 \mathrm{H}, \mathrm{CH}_{2}\right), 4.26$ (t, J=7.2 Hz, 2H, CH$)_{2}, 6.22$ (s, 1H, Ar), 6.70 (s, 1H, Ar), 7.09-7.16 (m, 2H, Ar), 7.25-7.28 (m, 1H, Ar), 7.36-7.40 (m, 1H, Ar). ${ }^{13} \mathrm{C} \mathrm{NMR}\left(\mathrm{CDCl}_{3}, 100 \mathrm{MHz}, \mathrm{TMS}\right) \delta$ 18.0, 21.0, 26.6, 64.7, 107.4 (d, $J=3.8 \mathrm{~Hz}), 116.0$ (d, $J=22.0 \mathrm{~Hz}), 116.5,117.2,124.1$ (d, $J=3.1 \mathrm{~Hz}), 124.4$, $129.1(\mathrm{~d}, J=8.3 \mathrm{~Hz}), 130.1(\mathrm{~d}, J=3.1 \mathrm{~Hz}), 159.8(\mathrm{~d}, J=248.3 \mathrm{~Hz}), 168.5,171.2 .{ }^{19} \mathrm{~F}$ NMR $(376$ $\left.\mathrm{MHz}, \mathrm{CDCl}_{3}, \mathrm{CFCl}_{3}\right) \delta-114.17-(-114.23)(\mathrm{m}) . \mathrm{IR}(\mathrm{EtOH}) \vee 3129,2951,2921,2847,1807,1734$, $1580,1513,1478,1459,1365,1238,1165,1124,1102,1033,1001,909,845,820,157,729,663$ $\mathrm{cm}^{-1}$. MS (ESI) $\mathrm{m} / z 323(\mathrm{M}+\mathrm{H})^{+}$. HRMS (ESI) calcd. for $\mathrm{C}_{16} \mathrm{H}_{20} \mathrm{FN}_{2} \mathrm{O}_{4}$ : 323.1402, Found: 323.1403 .

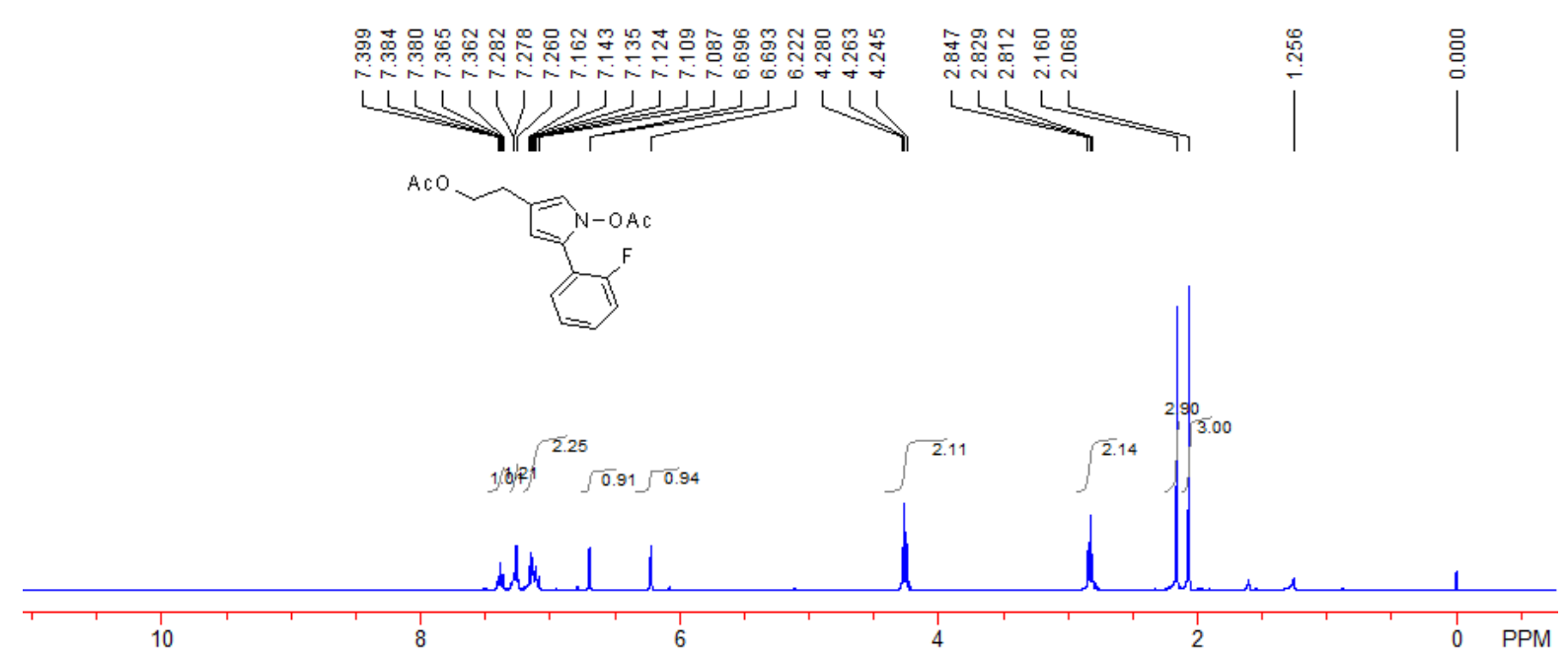




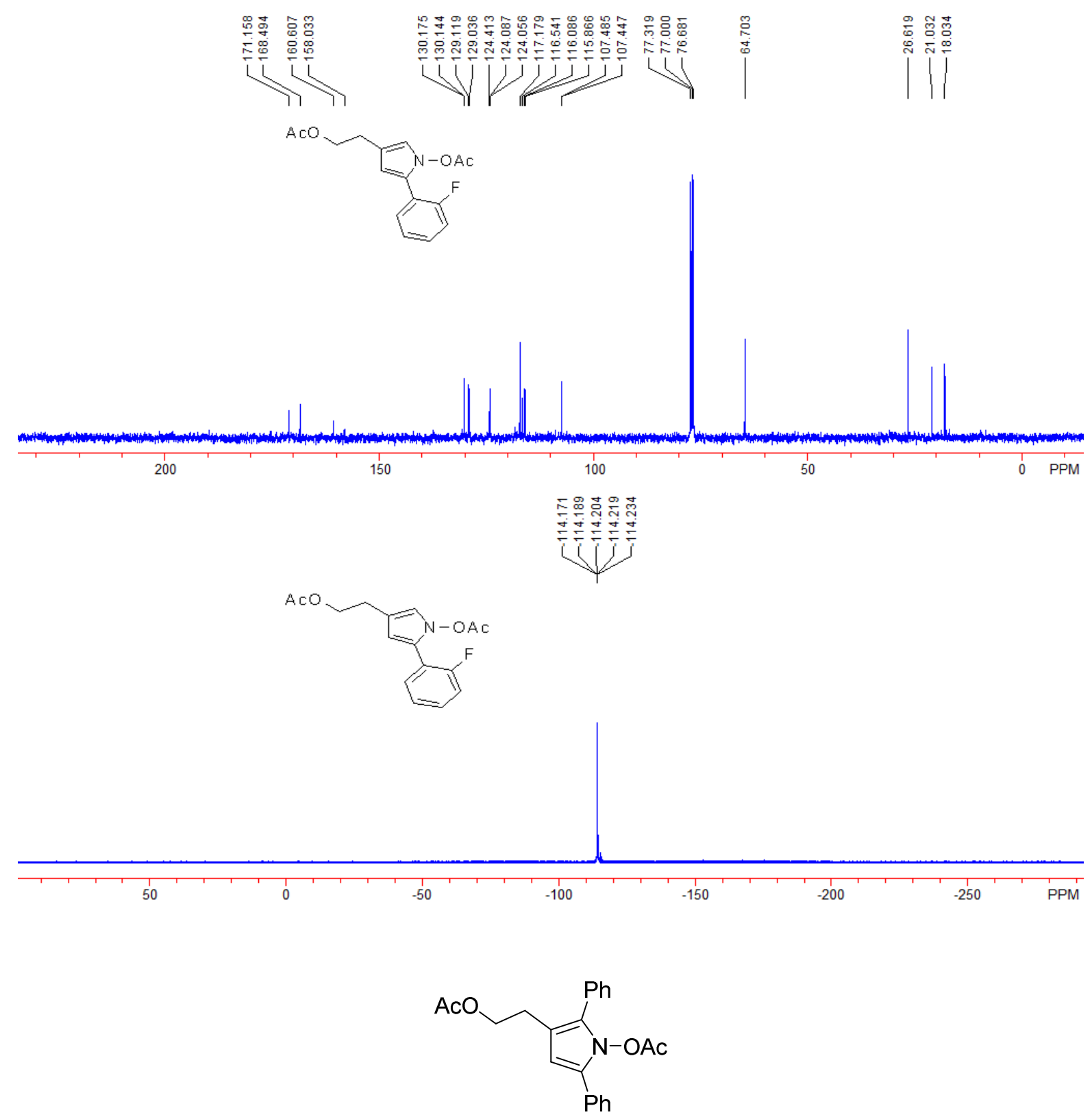

2-(1-acetoxy-2,5-diphenyl-1H-pyrrol-3-yl)ethyl acetate 5f: Yield: $41.0 \mathrm{mg}, 57 \%$; A colorless oil; ${ }^{1} \mathrm{H}$ NMR $\left(\mathrm{CDCl}_{3}, 400 \mathrm{MHz}, \mathrm{TMS}\right) \delta 1.90\left(\mathrm{~s}, 3 \mathrm{H}, \mathrm{CH}_{3}\right), 1.98\left(\mathrm{~s}, 3 \mathrm{H}, \mathrm{CH}_{3}\right), 2.87-2.93\left(\mathrm{~m}, 2 \mathrm{H}, \mathrm{CH}_{2}\right)$, 4.16-4.27 (m, 2H, $\mathrm{CH}_{2}$ ), 6.33 (s, 1H, Ar), 7.26-7.45 (m, 8H, Ar), 7.53-7.55 (m, 2H, Ar). ${ }^{13} \mathrm{C}$ NMR $\left(\mathrm{CDCl}_{3}, 100 \mathrm{MHz}, \mathrm{TMS}\right) \delta 18.0,21.0,25.9,64.7,105.8,114.6,126.7,127.0,127.5,128.4,128.6$, 128.8, 129.4, 129.7, 130.5, 131.7, 168.2, 171.1. IR (EtOH) v 3057, 3033, 2956, 2915, 2845, 1804, 1736, 1603, 1493, 1364, 1232, 1165, 1030, 1006, 846, 756, $697 \mathrm{~cm}^{-1}$. MS (ESI) $\mathrm{m} / z 364(\mathrm{M}+\mathrm{H})^{+}$. HRMS (ESI) calcd. for $\mathrm{C}_{22} \mathrm{H}_{22} \mathrm{NO}_{4}: 364.1543$, Found: 364.1544. 


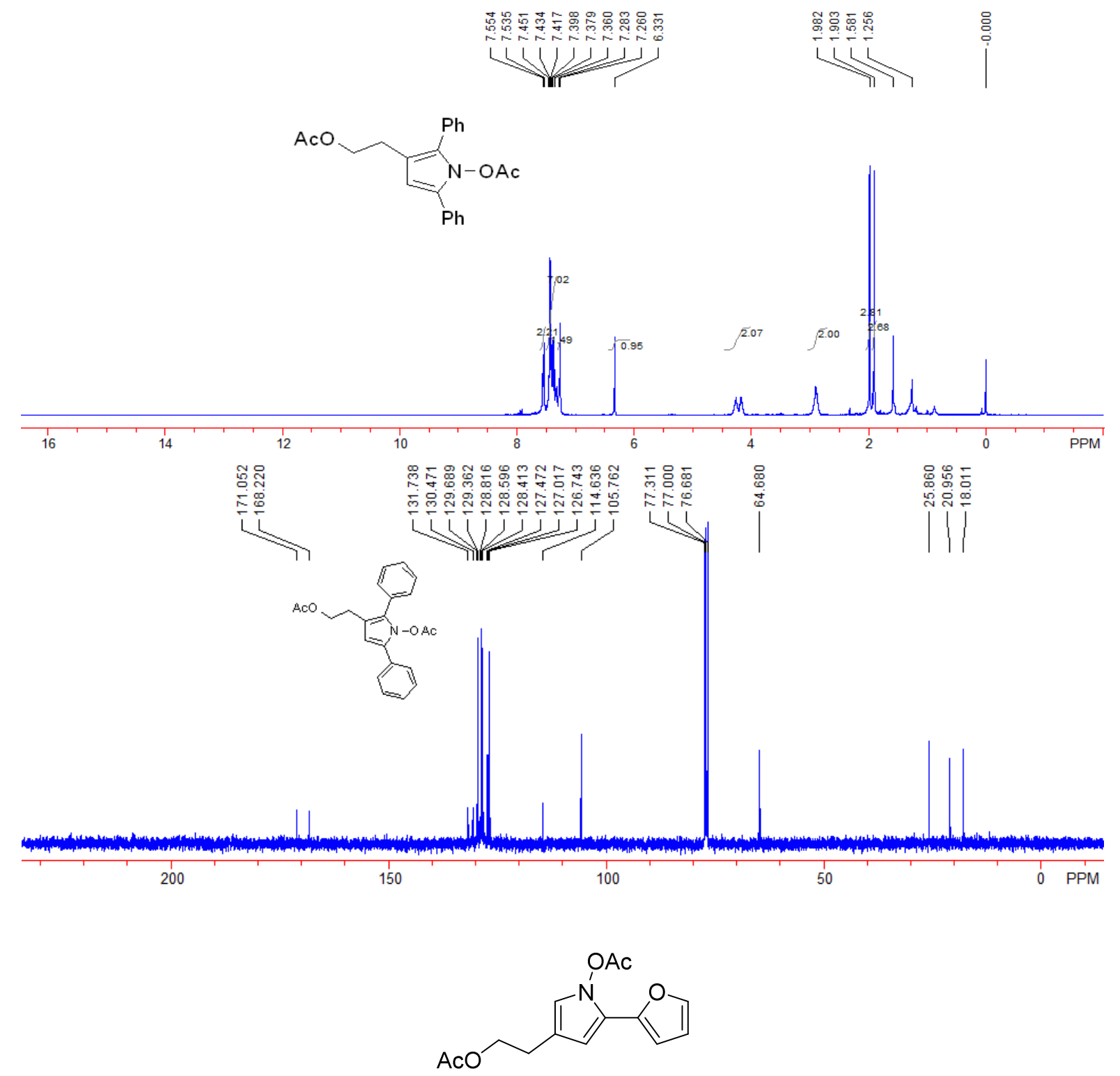

2-(1-acetoxy-5-(furan-2-yl)-1H-pyrrol-3-yl)ethyl acetate 5g: Yield: 33.4 mg, 60\%; A colorless oil; ${ }^{1} \mathrm{H} \mathrm{NMR}\left(\mathrm{CDCl}_{3}, 400 \mathrm{MHz}, \mathrm{TMS}\right) \delta 2.07\left(\mathrm{~s}, 3 \mathrm{H}, \mathrm{CH}_{3}\right), 2.33\left(\mathrm{~s}, 3 \mathrm{H}, \mathrm{CH}_{3}\right), 2.80(\mathrm{t}, J=7.2 \mathrm{~Hz}, 2 \mathrm{H}$, $\left.\mathrm{CH}_{2}\right), 4.24$ (t, $\left.J=7.2 \mathrm{~Hz}, 2 \mathrm{H}, \mathrm{CH}_{2}\right), 6.28-6.30$ (m, 2H, Ar), 6.40-6.41 (m, 1H, Ar), 6.60 (s, 1H, Ar), 7.37 (s, 1H, Ar). ${ }^{13} \mathrm{C} \mathrm{NMR}\left(\mathrm{CDCl}_{3}, 100 \mathrm{MHz}, \mathrm{TMS}\right) \delta$ 18.2, 21.0, 26.5, 64.6, 104.3, 105.0, 111.1, 116.48, 116.49, 121.6, 141.3, 145.2, 168.6, 171.1. IR (EtOH) v 3126, 2953, 2917, 2847, 1809, 1732, 1364, 1239, 1165, 1123, 1031, 1002, 839, 793, 777, 734, $670 \mathrm{~cm}^{-1}$. MS (ESI) m/z $278(\mathrm{M}+\mathrm{H})^{+}$. HRMS (ESI) calcd. for $\mathrm{C}_{14} \mathrm{H}_{16} \mathrm{NO}_{5}$ : 278.1023, Found: 278.1025 . 

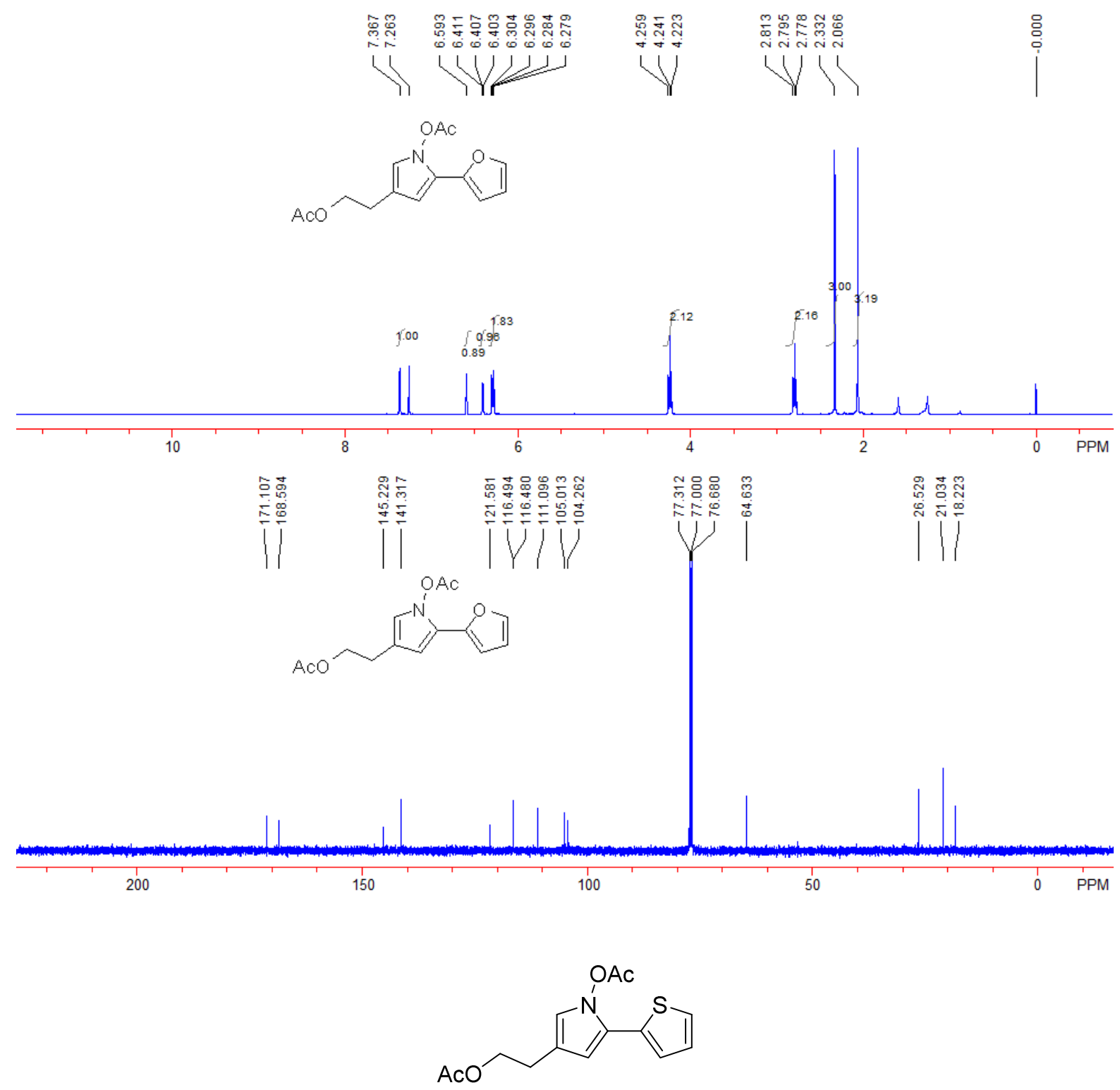

2-(1-acetoxy-5-(thiophen-2-yl)-1H-pyrrol-3-yl)ethyl acetate 5h: Yield: $38.8 \mathrm{mg}, 66 \%$; A colorless oil; ${ }^{1} \mathrm{H}$ NMR $\left(\mathrm{CDCl}_{3}, 400 \mathrm{MHz}, \mathrm{TMS}\right) \delta 2.07\left(\mathrm{~s}, 3 \mathrm{H}, \mathrm{CH}_{3}\right), 2.31\left(\mathrm{~s}, 3 \mathrm{H}, \mathrm{CH}_{3}\right), 2.80$ (t, $J=7.2 \mathrm{~Hz}$, $\left.2 \mathrm{H}, \mathrm{CH}_{2}\right), 4.25$ (t, $\left.J=7.2 \mathrm{~Hz}, 2 \mathrm{H}, \mathrm{CH}_{2}\right), 6.23(\mathrm{~d}, J=2.0 \mathrm{~Hz}, 1 \mathrm{H}, \mathrm{Ar}), 6.61$ (d, $\left.J=2.0 \mathrm{~Hz}, 1 \mathrm{H}, \mathrm{Ar}\right)$, 7.01-7.03 (m, 1H, Ar) , 7.09 (d, $J=2.4 \mathrm{~Hz}, 1 \mathrm{H}, \mathrm{Ar}), 7.20-7.22(\mathrm{~m}, 1 \mathrm{H}, \mathrm{Ar}) .{ }^{13} \mathrm{C} \mathrm{NMR}\left(\mathrm{CDCl}_{3}, 100\right.$ MHz, TMS) $\delta 18.4,21.1,26.6,64.6,105.0,116.46,116.50,123.7,124.1,124.3,127.3,131.7$, 168.6, 171.1. IR $(\mathrm{EtOH}) \vee$ 3098, 2951, 2920, 2847, 1810, 1732, 1419, 1364, 1238, 1160, 1121, 1032, 943, 827, 795, $698 \mathrm{~cm}^{-1}$. MS (ESI) $\mathrm{m} / z 294(\mathrm{M}+\mathrm{H})^{+}$. HRMS (ESI) calcd. for $\mathrm{C}_{14} \mathrm{H}_{16} \mathrm{NO}_{4} \mathrm{~S}$ : 294.0795, Found: 294.0797. 


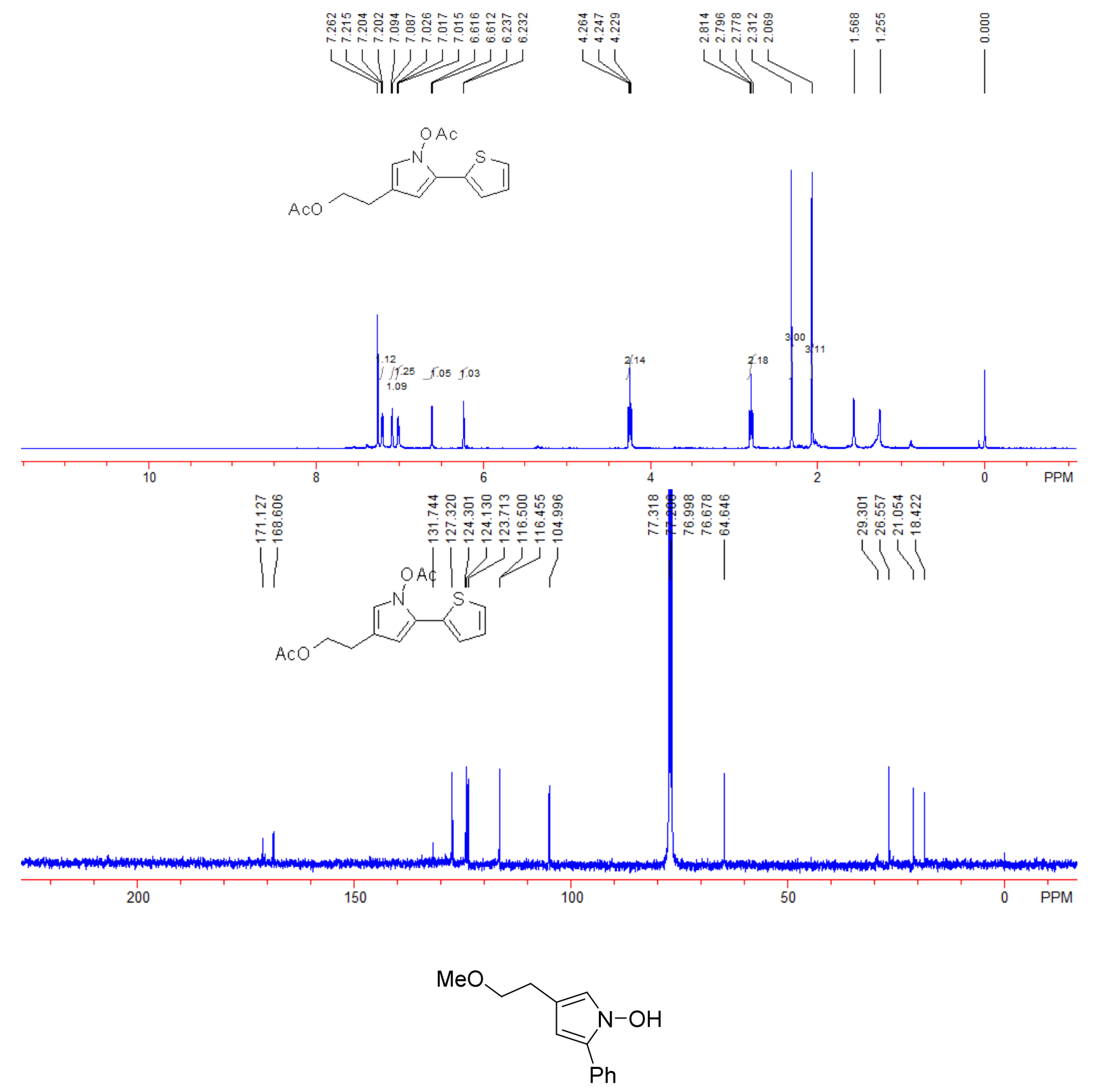

2-(1-acetoxy-5-(p-tolyl)-1H-pyrrol-3-yl)ethyl acetate 6a: Yield: $41.4 \mathrm{mg}, 95 \%$; A red oil; ${ }^{1} \mathrm{H}$ NMR $\left(\mathrm{CDCl}_{3}, 400 \mathrm{MHz}, \mathrm{TMS}\right) \delta 2.06\left(\mathrm{~s}, 3 \mathrm{H}, \mathrm{CH}_{3}\right), 2.18\left(\mathrm{~s}, 3 \mathrm{H}, \mathrm{CH}_{3}\right), 2.35\left(\mathrm{~s}, 3 \mathrm{H}, \mathrm{CH}_{3}\right), 2.81$ (t, $J=7.2$ $\left.\mathrm{Hz}, 2 \mathrm{H}, \mathrm{CH}_{2}\right), 4.25\left(\mathrm{t}, J=7.2 \mathrm{~Hz}, 2 \mathrm{H}, \mathrm{CH}_{2}\right), 6.13(\mathrm{~d}, J=2.0 \mathrm{~Hz}, 1 \mathrm{H}, \mathrm{Ar}), 6.62(\mathrm{~d}, J=2.0 \mathrm{~Hz}, 1 \mathrm{H}$, Ar), $7.16(\mathrm{~d}, J=8.0 \mathrm{~Hz}, 2 \mathrm{H}, \mathrm{Ar}), 7.33$ (d, $J=8.0 \mathrm{~Hz}, 2 \mathrm{H}, \mathrm{Ar}) .{ }^{13} \mathrm{C} \mathrm{NMR}\left(\mathrm{CDCl}_{3}, 100 \mathrm{MHz}, \mathrm{TMS}\right)$ $\delta 18.2,21.0,21.2,26.6,64.7,104.8,116.3,116.5,126.7,127.6,129.3,130.9,136.8,168.7,171.1$. IR $(\mathrm{EtOH}) \vee 3231,2971,2926,1666,1600,1508,1490,1442,1424,1310,1087,1046,986,950$, 938, 924, 879, 846, 756, $691 \mathrm{~cm}^{-1}$. MS (ESI) $\mathrm{m} / z 218(\mathrm{M}+\mathrm{H})^{+}$. HRMS (ESI) calcd. for $\mathrm{C}_{13} \mathrm{H}_{16} \mathrm{NO}_{2}$ : 218.1176, Found: 218.1176. 


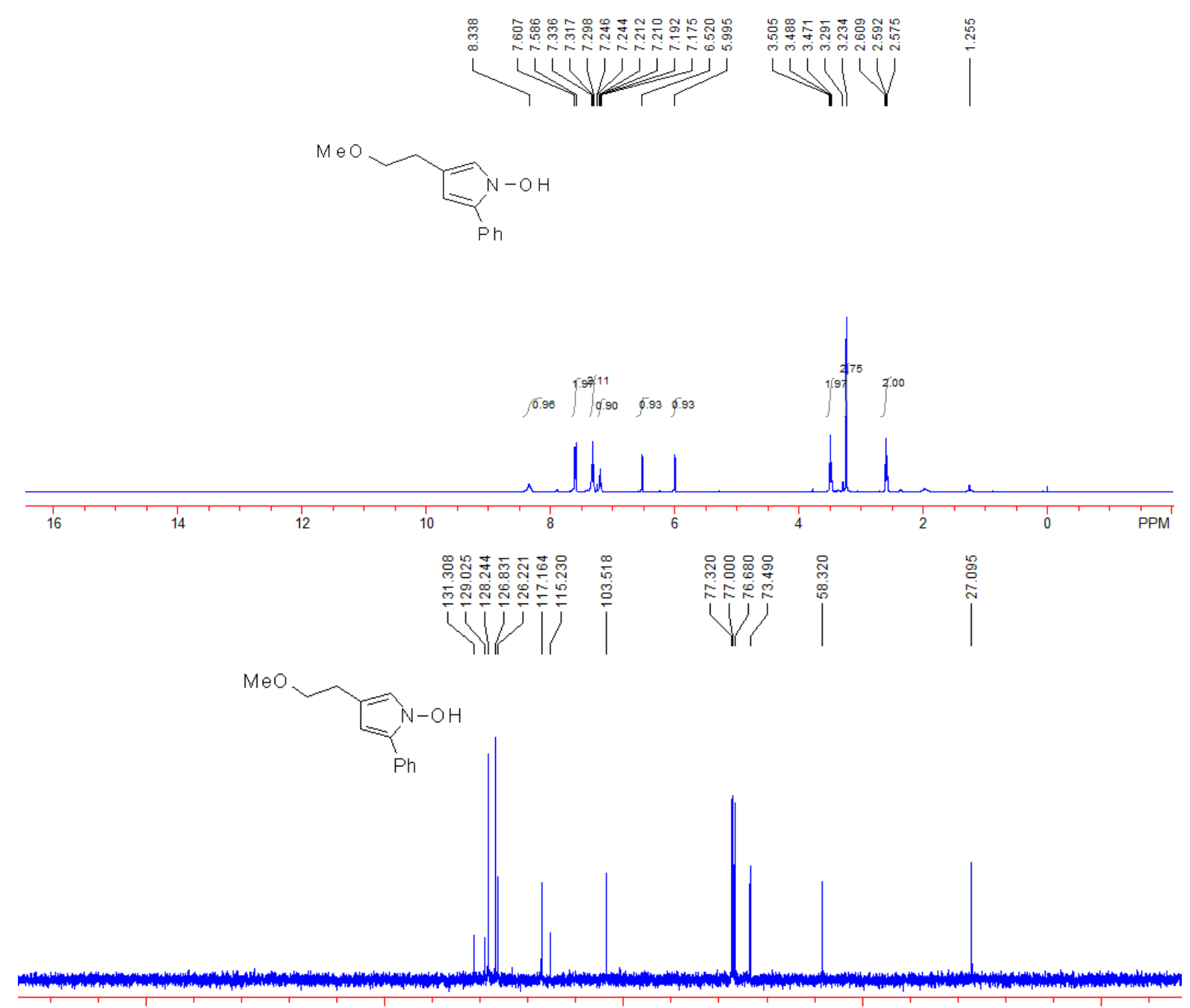

200

150

100

50

PPM

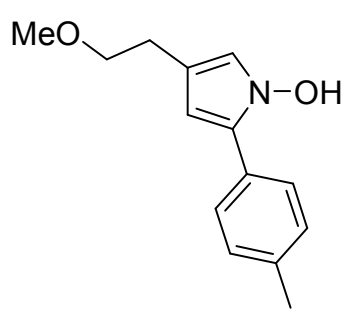

4-(2-methoxyethyl)-2-(p-tolyl)-1H-pyrrol-1-ol 6b: Yield: $42.2 \mathrm{mg}, 95 \%$; A red oil; ${ }^{1} \mathrm{H} \mathrm{NMR}\left(\mathrm{CDCl}_{3}\right.$, $400 \mathrm{MHz}, \mathrm{TMS}) \delta 2.64\left(\mathrm{~s}, 3 \mathrm{H}, \mathrm{CH}_{3}\right), 2.18\left(\mathrm{~s}, 3 \mathrm{H}, \mathrm{CH}_{3}\right), 2.35\left(\mathrm{~s}, 3 \mathrm{H}, \mathrm{CH}_{3}\right), 2.81(\mathrm{t}, J=7.2 \mathrm{~Hz}, 2 \mathrm{H}$, $\left.\mathrm{CH}_{2}\right), 4.25\left(\mathrm{t}, J=7.2 \mathrm{~Hz}, 2 \mathrm{H}, \mathrm{CH}_{2}\right), 6.13(\mathrm{~d}, J=2.0 \mathrm{~Hz}, 1 \mathrm{H}, \mathrm{Ar}), 6.62$ (d, $\left.J=2.0 \mathrm{~Hz}, 1 \mathrm{H}, \mathrm{Ar}\right), 7.16$ $(\mathrm{d}, J=8.0 \mathrm{~Hz}, 2 \mathrm{H}, \mathrm{Ar}), 7.33(\mathrm{~d}, J=8.0 \mathrm{~Hz}, 2 \mathrm{H}, \mathrm{Ar}) .{ }^{13} \mathrm{C} \mathrm{NMR}\left(\mathrm{CDCl}_{3}, 100 \mathrm{MHz}, \mathrm{TMS}\right) \delta 18.2$, 21.0, 21.2, 26.6, 64.7, 104.8, 116.3, 116.5, 126.7, 127.6, 129.3, 130.9, 136.8, 168.7, 171.1. IR $(\mathrm{EtOH}) \vee 3196,2922,2861,1521,1449,1379,1221,1191,1097,1047,999,948,820,783 \mathrm{~cm}^{-1}$. MS (ESI) $m / z 232(\mathrm{M}+\mathrm{H})^{+}$. HRMS (ESI) calcd. for $\mathrm{C}_{14} \mathrm{H}_{18} \mathrm{NO}_{2}$ : 232.1332, Found: 232.1333. 


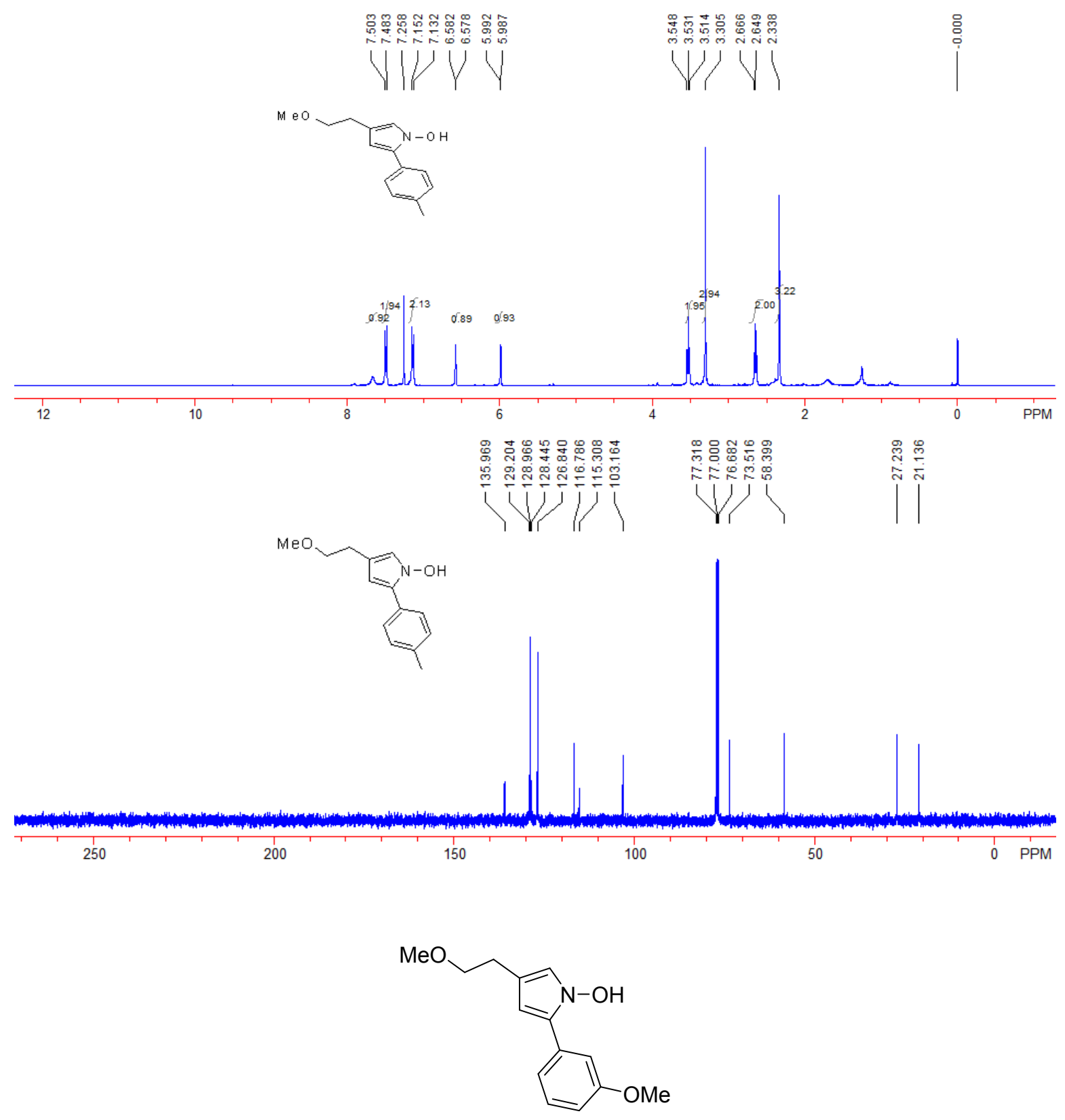

2-(1-acetoxy-5-(p-tolyl)-1H-pyrrol-3-yl)ethyl acetate 6c: Yield: 46.0 mg, 94\%; A red oil; ${ }^{1} \mathrm{H}$ NMR $\left(\mathrm{CDCl}_{3}, 400 \mathrm{MHz}, \mathrm{TMS}\right) \delta 2.64\left(\mathrm{t}, J=6.8 \mathrm{~Hz}, 2 \mathrm{H}, \mathrm{CH}_{2}\right), 3.29\left(\mathrm{~s}, 3 \mathrm{H}, \mathrm{CH}_{3}\right), 3.53(\mathrm{t}, J=6.8 \mathrm{~Hz}, 2 \mathrm{H}$, $\left.\mathrm{CH}_{2}\right), 3.79$ (s, 3H, $\left.\mathrm{CH}_{3}\right), 6.03$ (s, 1H, Ar), 6.58 (s, 1H, Ar), 6.75-6.77 (m, 1H, Ar), 7.18-7.27 (m, 3H, Ar), 7.96 (br, 1H, OH). ${ }^{13} \mathrm{C} \mathrm{NMR}\left(\mathrm{CDCl}_{3}, 100 \mathrm{MHz}, \mathrm{TMS}\right) \delta$ 27.2, 55.2, 58.4, 73.4, 103.9, 111.9, 112.4, 115.5, 117.3, 119.5, 128.8, 129.3, 132.6, 159.4. IR (EtOH) v 3167, 2932, 2833, 1603, 1578, $1504,1479,1463,1382,1314,1284,1251,1197,1166,1095,1047,1015,992,949,859,776,734$, $691 \mathrm{~cm}^{-1}$. MS (ESI) $\mathrm{m} / z 248(\mathrm{M}+\mathrm{H})^{+}$. HRMS (ESI) calcd. for $\mathrm{C}_{14} \mathrm{H}_{18} \mathrm{NO}_{3}$ : 248.1281, Found: 248.1283. 


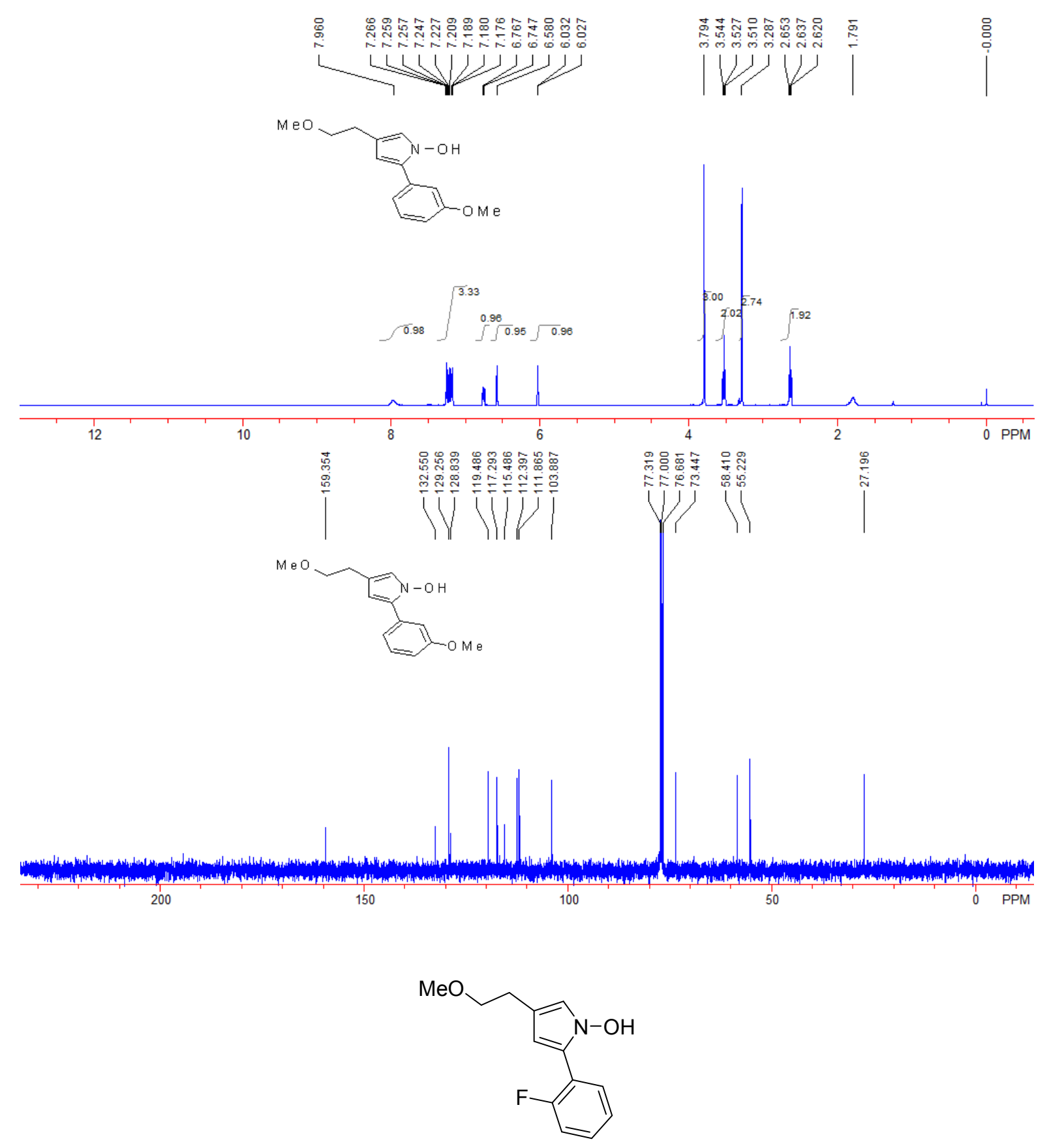

2-(2-fluorophenyl)-4-(2-methoxyethyl)-1H-pyrrol-1-ol 6d: Yield: $42.5 \mathrm{mg}, 90 \%$; A red oil; ${ }^{1} \mathrm{H}$ NMR $\left(\mathrm{CDCl}_{3}, 400 \mathrm{MHz}, \mathrm{TMS}\right) \delta 2.70\left(\mathrm{t}, J=6.8 \mathrm{~Hz}, 2 \mathrm{H}, \mathrm{CH}_{2}\right), 3.33\left(\mathrm{~s}, 3 \mathrm{H}, \mathrm{CH}_{3}\right), 3.56(\mathrm{t}, J=6.8$ $\left.\mathrm{Hz}, 2 \mathrm{H}, \mathrm{CH}_{2}\right), 6.07$ (s, 1H, Ar), 6.73 (s, 1H, Ar), 7.11-7.28 (m, 3H, Ar), 7.47 (br, 1H, OH), 7.51-7.56 (m, 2H, Ar). ${ }^{13} \mathrm{C} \mathrm{NMR}\left(\mathrm{CDCl}_{3}, 100 \mathrm{MHz}, \mathrm{TMS}\right) \delta 27.3,58.5,73.5,77.2,106.1(\mathrm{~d}, J=$ $3.0 \mathrm{~Hz}), 115.7(\mathrm{~d}, J=22.8 \mathrm{~Hz}), 116.0,117.2,119.3$ (d, $J=13.7 \mathrm{~Hz}), 123.8,124.2(\mathrm{~d}, J=3.0 \mathrm{~Hz})$,

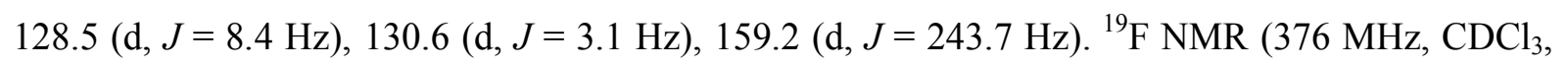
$\left.\mathrm{CFCl}_{3}\right) \delta-115.17-(-115.19)(\mathrm{m}) . \mathrm{IR}(\mathrm{EtOH}) \vee 3187,2919,1682,1613,1576,1508,1476,1456$, 
1383, 1265, 1225, 1100, 1036, 1001, 945, 818, 756, 735, $702 \mathrm{~cm}^{-1}$. MS (ESI) m/z $236(\mathrm{M}+\mathrm{H})^{+}$. HRMS (ESI) calcd. for $\mathrm{C}_{13} \mathrm{H}_{15} \mathrm{FNO}_{2}: 236.1081$, Found: 236.1083.

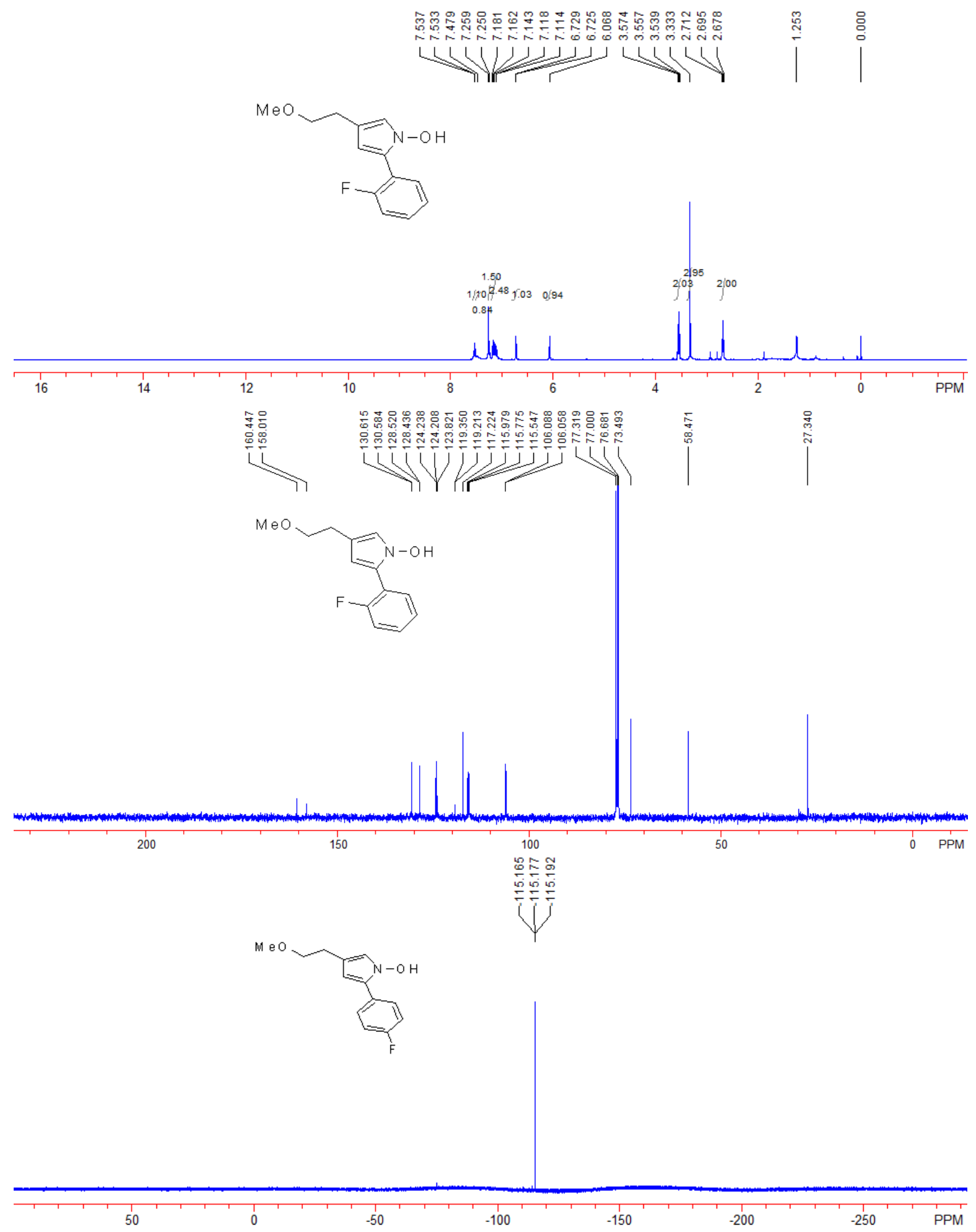




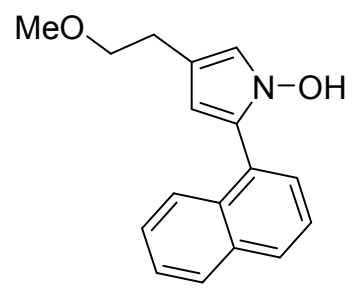

2-(1-acetoxy-5-(p-tolyl)-1H-pyrrol-3-yl)ethyl acetate 6e: Yield: $48.1 \mathrm{mg}, 92 \%$; A red oil; ${ }^{1} \mathrm{H}$ NMR $\left(\mathrm{CDCl}_{3}, 400 \mathrm{MHz}, \mathrm{TMS}\right) \delta 2.66\left(\mathrm{t}, J=6.8 \mathrm{~Hz}, 2 \mathrm{H}, \mathrm{CH}_{2}\right), 3.16\left(\mathrm{~s}, 3 \mathrm{H}, \mathrm{CH}_{3}\right), 3.51(\mathrm{t}, J=6.8 \mathrm{~Hz}, 2 \mathrm{H}$, $\mathrm{CH}_{2}$ ), 6.00 (s, 1H, Ar), 6.67 (s, 1H, Ar), 7.45-7.53 (m, 4H, Ar), 7.59 (br, 1H, OH), 7.81-7.87 (m, 2H, $\mathrm{Ar})$, 8.00-8.02 (m, 1H, Ar). ${ }^{13} \mathrm{C} \mathrm{NMR}\left(\mathrm{CDCl}_{3}, 100 \mathrm{MHz}, \mathrm{TMS}\right) \delta 27.3,58.3,73.5,106.1,115.3$, $115.9,125.2,125.8,126.18,126.24,127.7,128.0,128.2$, 128.4, 129.0, 132.3, 133.6. IR (EtOH) v $3180,3049,2971,2926,2896,2860,2827,1687,1593,1508,1447,1386,1259,1228,1192,1099$, 1045, 1018, 988, 954, 878, 775, 738, $676 \mathrm{~cm}^{-1}$. MS (ESI) $\mathrm{m} / z 268(\mathrm{M}+\mathrm{H})^{+}$. HRMS (ESI) calcd. for $\mathrm{C}_{17} \mathrm{H}_{18} \mathrm{NO}_{2}$ : 268.1332, Found: 268.1333 .

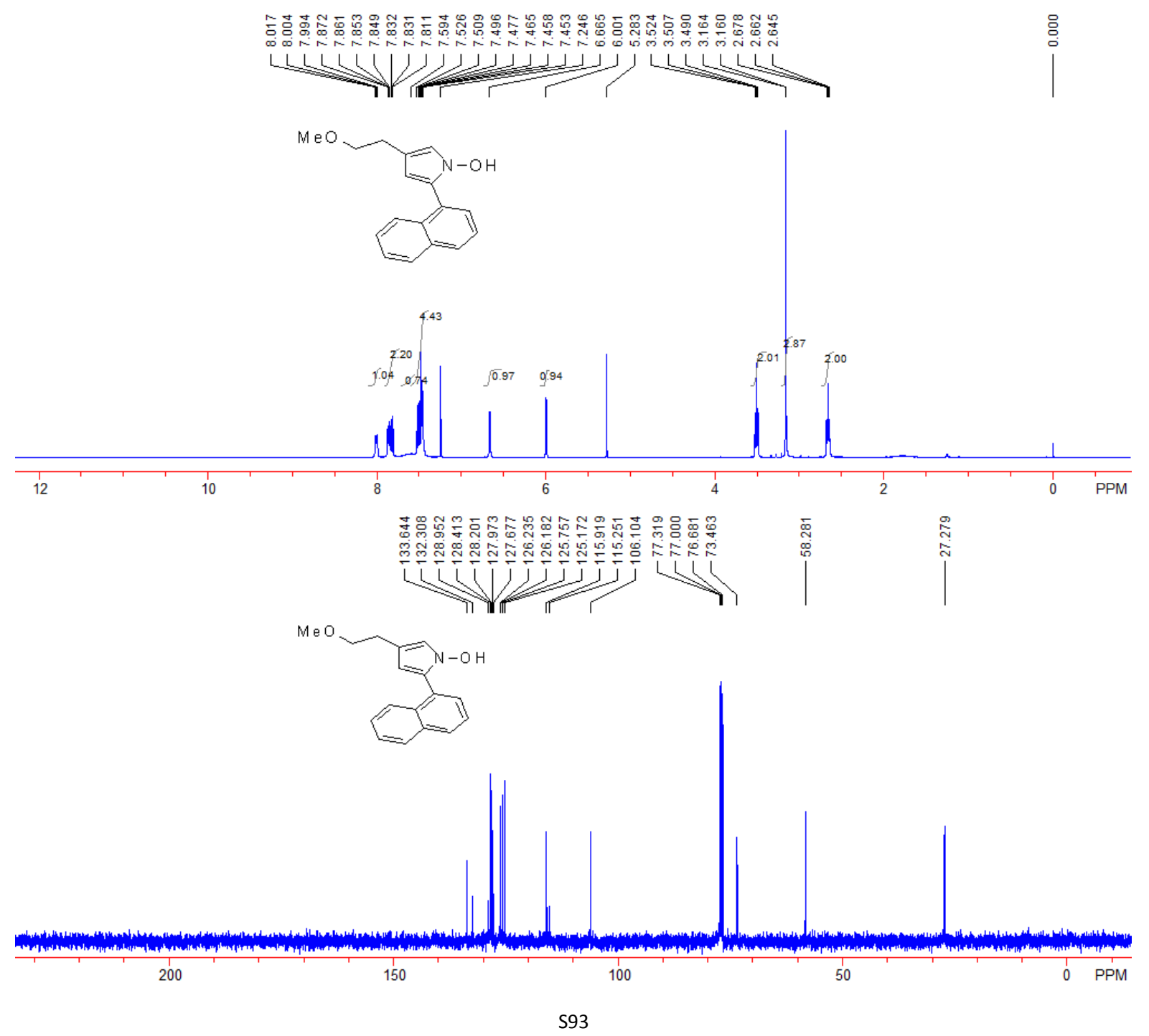




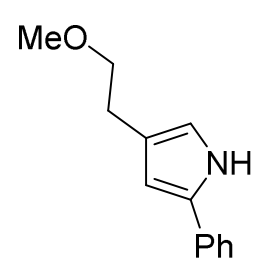

4-(2-methoxyethyl)-2-phenyl-1H-pyrrole 8b: Yield: $89.9 \mathrm{mg}$, 89\%; A colorless oil; ${ }^{1} \mathrm{H}$ NMR $\left(\mathrm{CDCl}_{3}, 400 \mathrm{MHz}, \mathrm{TMS}\right) \delta 2.78\left(\mathrm{t}, J=7.2 \mathrm{~Hz}, 2 \mathrm{H}, \mathrm{CH}_{2}\right), 3.37$ (s, 3H, $\left.\mathrm{CH}_{3}\right), 3.60$ (t, $J=7.2 \mathrm{~Hz}, 2 \mathrm{H}$, $\mathrm{CH}_{2}$ ), 6.39 (s, 1H, Ar), 6.61 (s, 1H, Ar), 7.14-7.17 (m, 1H, Ar), 7.29-7.32 (m, 2H, Ar), 7.39-7.41 (m, 2H, Ar), 8.41 (br, 1H, NH). ${ }^{13} \mathrm{C}$ NMR ( $\left.\mathrm{CDCl}_{3}, 100 \mathrm{MHz}, \mathrm{TMS}\right) \delta$ 27.4, 58.5, 73.7, 106.3, 116.8, 122.0, 123.6, 125.9, 128.7, 131.9, 132.7. IR (EtOH) v 3307, 2982, 2926, 2860, 2827, 1693, 1605, $1514,1476,1453,1382,1263,1192,1102,1052,982,955,932,904,804,760,732,692 \mathrm{~cm}^{-1}$. MS (ESI) $m / z 202(\mathrm{M}+\mathrm{H})^{+}$. HRMS (ESI) calcd. for $\mathrm{C}_{13} \mathrm{H}_{16} \mathrm{NO}: 202.1226$, Found: 202.1227 .

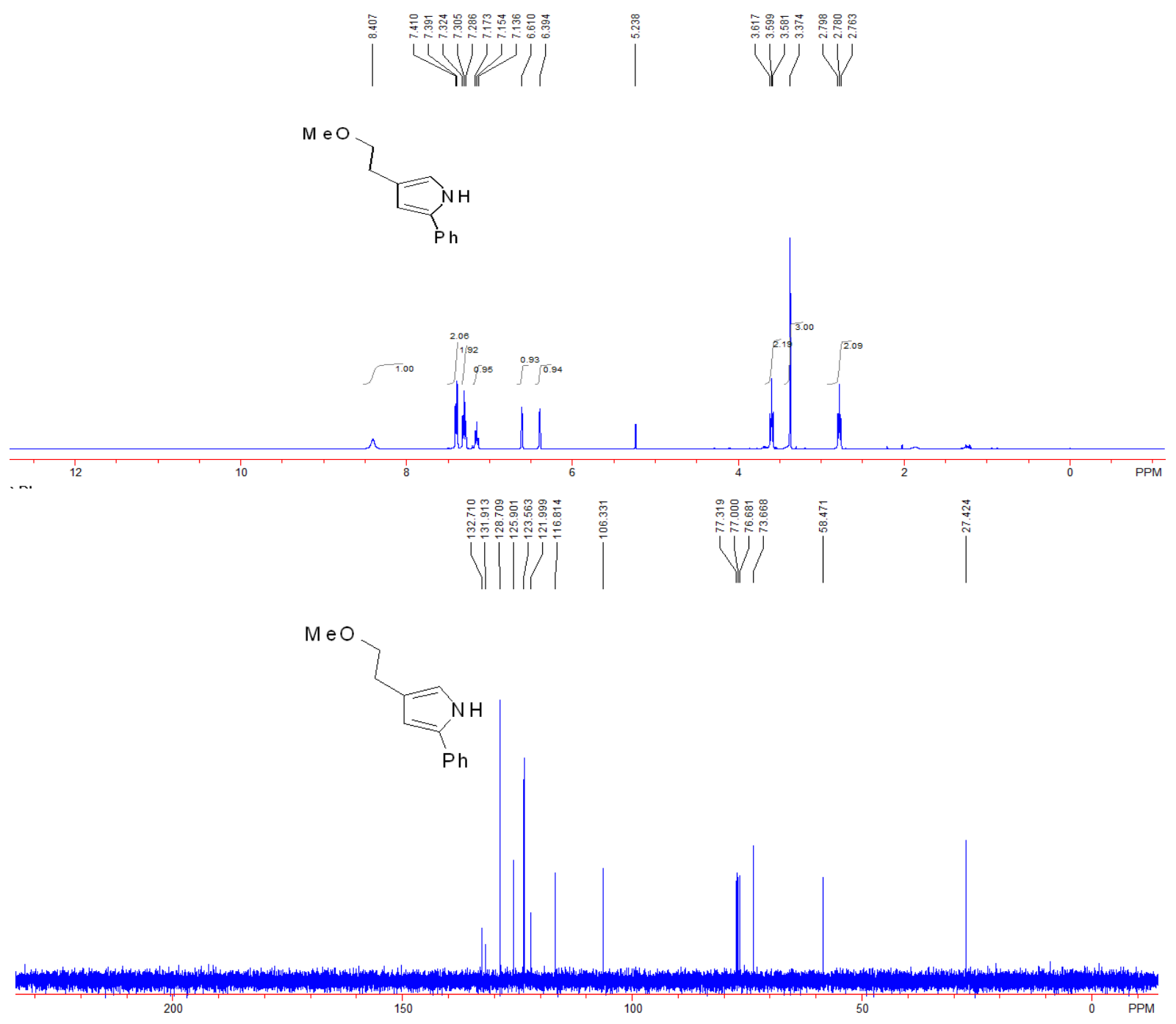




\section{References}

(1) Feuer, H.; Braunstein, D. M. J. Org. Chem. 1969, 34, 1817.

(2) Chen, G.-Q.; Zhang, X.-N.; Wei, Y.; Tang, X.-Y.; Shi, M. Angew. Chem., Int. Ed. 2014, 53, 8492.

(3) Hibino, S.; Sugino, E.; Adachi, Y.; Nomi, K.; Sato, K.; Fukumoto, K. Heterocycles 1989, 28, 275.

(4) Zhang, Y.; Zhang, J. Synlett 2012, 23, 1389.

(5) (a) Abramovitch, R. A.; Cue, B. W. J. Org. Chem. 1973, 38, 173. (b) Crayston, J. A.; Kakouris, C.; Walton, J. C. Magn. Reson. Chem. 1992, 30, 77.

(6) (a) Su, Y.; Zhou, H.; Chen, J.; Xu, J.; Wu, X.; Lin, A.; Yao, H. Org. Lett. 2014, 16, 4884. (b) Pei, K.; Jie, X.; Zhao, H.; Su, W. Eur. J. Org. Chem. 2014, 2014, 4230. 